\title{
Total Synthesis and Computational Investigations of Sesquiterpene-Tropolones Ameliorate Stereochemical Inconsistencies and Resolve an Ambiguous Biosynthetic Relationship
}

\author{
Christopher Y. Bemis,${ }^{\ddagger a b}$ Chad N. Ungarean, ${ }^{\ddagger a}, \mathrm{~b}$ Alexander S. Shved, ${ }^{\mathrm{a}, \mathrm{b}}$ Cooper S. \\ Jamieson, ${ }^{\mathrm{c}}$ Taehwan Hwang, ${ }^{\text {a,b }}$ Ken S. Lee, ${ }^{\text {a,b }}$ K. N. Houk, ${ }^{\mathrm{c}}$ and David Sarlah*a,b \\ ${ }^{a}$ Roger Adams Laboratory, Department of Chemistry, University of Illinois, Urbana, IL 61801, United States \\ ${ }^{b}$ Cancer Center at Illinois, University of Illinois, Urbana, IL 61801, United States \\ ${ }^{\text {c} D e p a r t m e n t ~ o f ~ C h e m i s t r y ~ a n d ~ B i o c h e m i s t r y, ~ U n i v e r s i t y ~ o f ~ C a l i f o r n i a, ~ L o s ~ A n g e l e s, ~ C A ~ 90095, ~ U n i t e d ~ S t a t e s ~}$ \\ correspondence to: David Sarlah, sarlah@illinois.edu
}

\section{Supporting Information}




\section{Table of Contents}

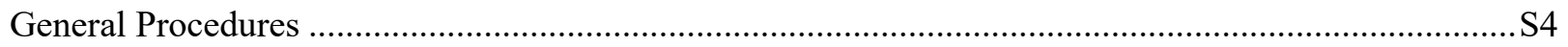

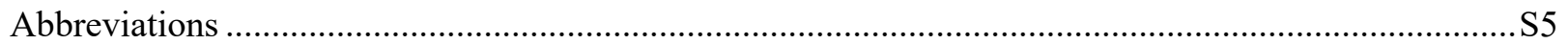

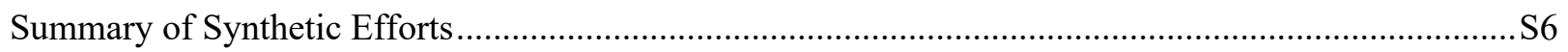

Experimental Procedures and Characterization Data ……………………………………………...... S15

Sequence for Synthesis of Tropolone $o$-QM precursors (19 and 20)

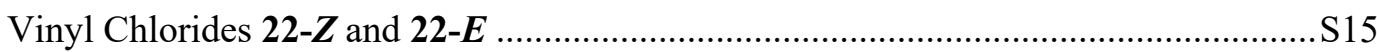

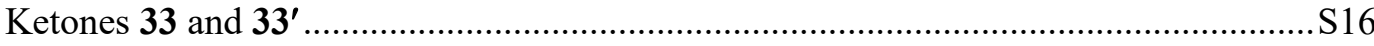

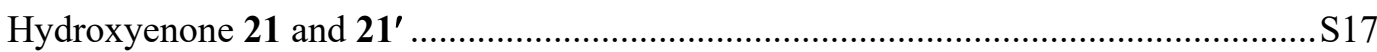

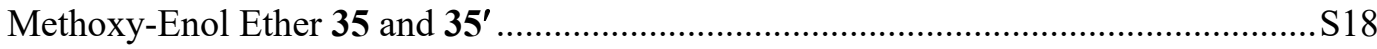

Tropolone Adduct 19 ……………………………………………………........ S20

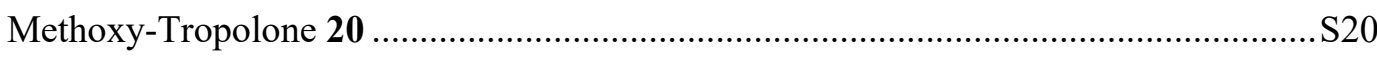

Sequence for Synthesis of (-)-10-Hydroxyhumulene 11
Hydroxyketone 40

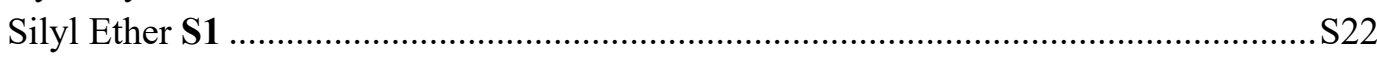

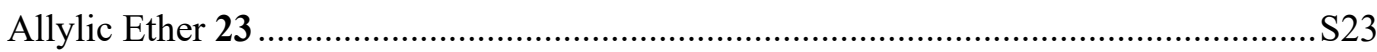

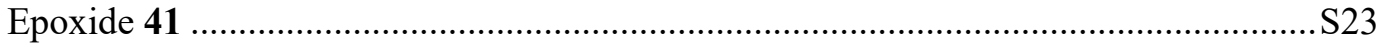

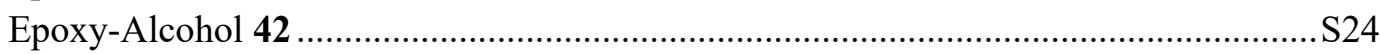

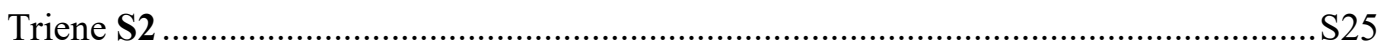

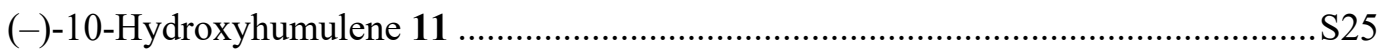

Sequence for Synthesis of Dehydroxyepolone B $\left(\mathbf{1 5}^{\prime}\right)$ and Dehydroxypycnidione (2')

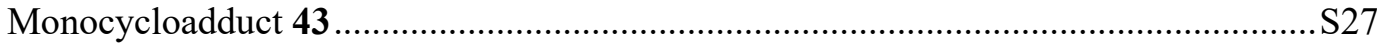

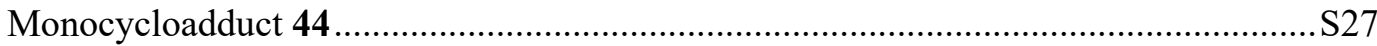

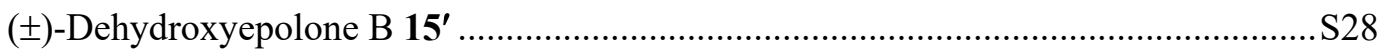

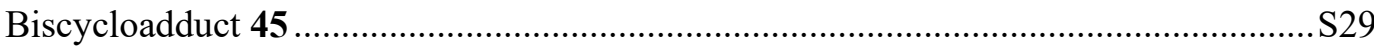

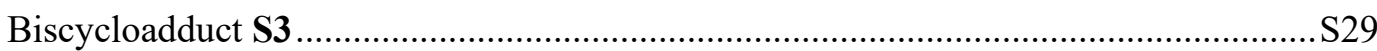

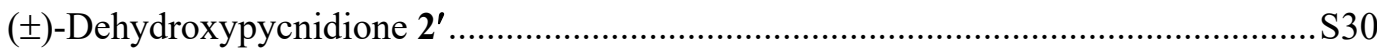

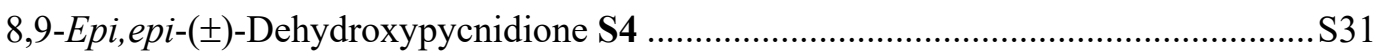

Sequence for Synthesis of (-)-Epolone B (ent-4), (+)-Isoepolone B (ent-16), 10-Epi-(-)-

Pycnidione (52), and 8,9-Epi,epi-(+)-Pycnidione (50)

Monocycloadduct 46

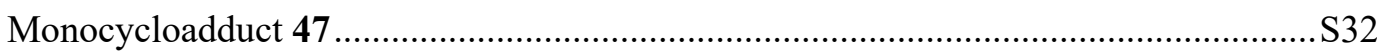

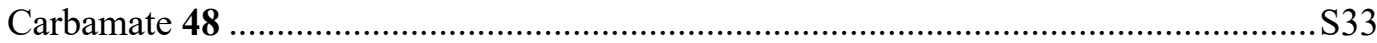

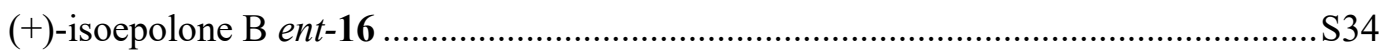

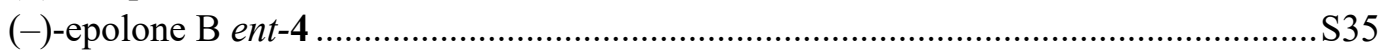

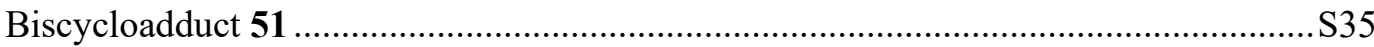

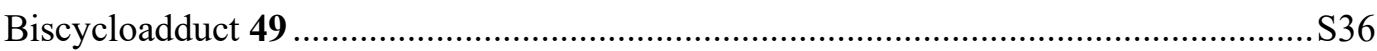

10-Epi-(-)-Pycnidione (52) ............................................................................... 337

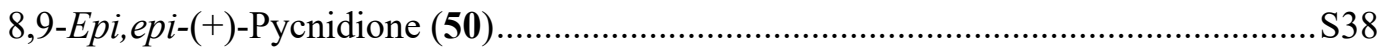

Computational Chemistry

General Computational Methods .................................................................................S39

Benchmark of Computational Methods ..................................................................... 44

Cartesian Coordinates of Calculated Structures................................................................ S44

Computational Methods for DFT-GIAO NMR Predictions ...........................................S78

Computational Methods for TDDFT-ECD Predictions ………………………………..... 883 


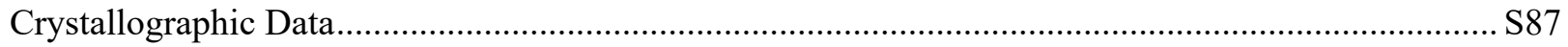

Comparisons of Natural and Synthetic Compounds

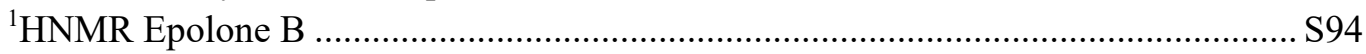

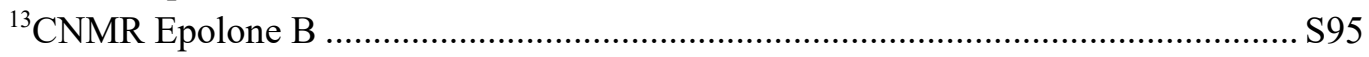

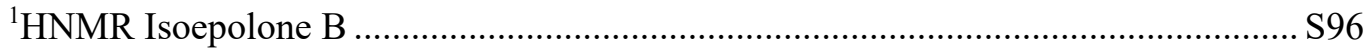

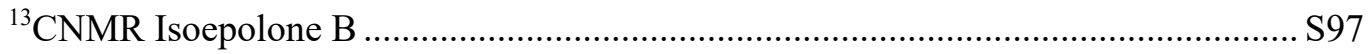

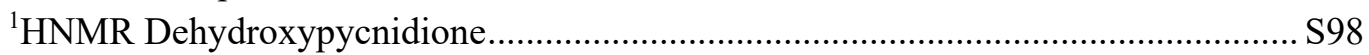

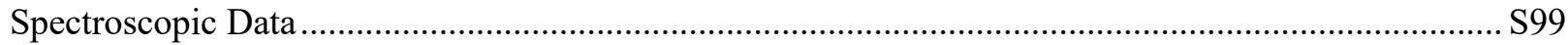

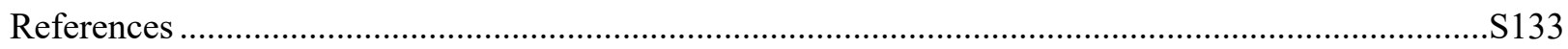




\section{General Procedures}

All chemicals were purchased from commercial suppliers and used as received, unless otherwise noted. $\mathrm{C}_{18}$-derivatized $\mathrm{SiO}_{2}$ was prepared according to the literature procedure. Diethyl ether (ACS grade), dichloromethane (ACS grade), tetrahydrofuran (HPLC grade), and toluene (ACS grade) were dried for reactions using the MB-SPS solvent purification system containing activated alumina manufactured by MBRAUN. Reaction temperatures correspond to the external temperature of the reaction vessel unless otherwise noted. Photochemical reactions were performed with LSE Lighting 15W 120V Compact Germicial UV (CFL/UV/MED) bulbs. Microwave reactions were performed in CEM Discover and Discover S Microwave Synthesis System.

Analytical thin-layer chromatography (TLC) was performed on Merck silica gel 60 F254 aluminum sheets. Visualization was accomplished with UV light and/or potassium permanganate $\left(\mathrm{KMnO}_{4}\right)$. Retention factor $\left(\mathrm{R}_{\mathrm{f}}\right)$ values reported were measured using a $10 \times 2 \mathrm{~cm}$ TLC plate in a developing chamber containing the solvent system described. Silicycle SiliaFlash ${ }^{\circledR} \mathrm{P} 60\left(\mathrm{SiO}_{2}, 40-63 \mu \mathrm{m}\right.$ particle size, 230-400 mesh) and aluminum oxide activated, basic, Brockmann I $\left(\mathrm{Al}_{2} \mathrm{O}_{3}, 58 \AA\right.$ pore size $)$ were used for flash column chromatography. Preparative HPLC was performed on a Shimadzu Prominence LC-20AP Preparative Liquid Chromatograph system with SPD-20A UV/VIS detector and a Kinetex ${ }^{\circledR} 5 \mu \mathrm{m}$ EVO $\mathrm{C}_{18} 100 \AA, 250$ x $100 \mathrm{~mm}$ column. UHPLC-MS experiments were performed on a Shimadzu Nexera XR LC-20AD XR liquid chromatograph system with a SPD-M30A diode array detector and a Kinetex ${ }^{\circledR} 1.7 \mu \mathrm{m}$ EVO $\mathrm{C}_{18}$ $100 \AA$, 50 x $2.1 \mathrm{~mm}$ column.

${ }^{1} \mathrm{H}$ NMR spectra were obtained at $500 \mathrm{MHz},{ }^{13} \mathrm{C}$ NMR were obtained at $126 \mathrm{MHz},{ }^{19} \mathrm{~F}$ NMR were obtained at $470 \mathrm{MHz}$. NMR spectra were recorded using a Bruker $500 \mathrm{MHz}$ spectrometer and were referenced to residual chloroform $\left(7.26 \mathrm{ppm},{ }^{1} \mathrm{H} ; 77.0 \mathrm{ppm},{ }^{13} \mathrm{C}\right)$ or residual dichloromethane (5.32 ppm, $\left.{ }^{1} \mathrm{H} ; 53.8 \mathrm{ppm},{ }^{13} \mathrm{C}\right)$. Chemical shifts are reported in parts per million (ppm) and multiplicities are indicated as: s (singlet), d (doublet), $\mathrm{t}$ (triplet), $\mathrm{q}$ (quartet), $\mathrm{p}$ (pentet), $\mathrm{m}$ (multiplet), and br (broad). Coupling constants, $J$, are reported in Hertz. Mass spectrometry (MS) was performed by the University of Illinois Mass Spectrometry Laboratory. Electron Impact (EI+) spectra were performed at $70 \mathrm{eV}$ using methane as the carrier gas, with time-of-flight (TOF) mass analyzer. Electrospray ionization (ES+) spectra were performed using a time-of-flight (TOF) mass analyzer. Data are reported in the form of $\mathrm{m} / \mathrm{z}$. Infrared (IR) spectra were measured neat on a Perkin-Elmer Spectrum Two FT-IR ATR spectrometer. Peaks are reported in $\mathrm{cm}^{-1}$ with indicated relative intensities: $\mathrm{s}$ (strong, $0-33 \% \mathrm{~T}$ ); $\mathrm{m}$ (medium, $34-66 \% \mathrm{~T}$ ), w (weak, 67 $100 \% \mathrm{~T}$ ), and br (broad). Melting points were measured on a Buchi B-540 melting point apparatus and are uncorrected. Optical rotations were recorded on a Jasco P-2000 polarimeter at $589 \mathrm{~nm}$ and are reported in units of $10^{-1}\left(\mathrm{deg} \mathrm{cm}^{2} \mathrm{~g}^{-1}\right)$. The X-ray diffraction experiments were conducted using a Bruker D8 Venture/Photon 100 diffractometer. Using Olex2 the structures were solved with ShelXT structure solution 
program using the intrinsic phasing solution method and the XT refinement package using least squared minimization.

\begin{abstract}
Abbreviations
$\mathrm{THF}=$ tetrahydrofuran, $\mathrm{DMF}=N, N$-Dimethylformamide, $\mathrm{DMSO}=$ Dimethylsulfoxide, $\mathrm{HMDS}=$ Hexamethyldisilazane, KHMDS $=$ Potassium bis(trimethylsilyl)amide, TBAF $=$ Tert- $n$-butylammonium fluoride, $\mathrm{TMSCl}=$ Trimethylsilylchloride, $\mathrm{TBSCl}=$ tert-butyldimethylsilyl chloride, $\mathrm{BHT}=$ Butylated hydroxytoluene.
\end{abstract}




\section{Summary of Synthetic Efforts}

Tropolone Synthetic Studies

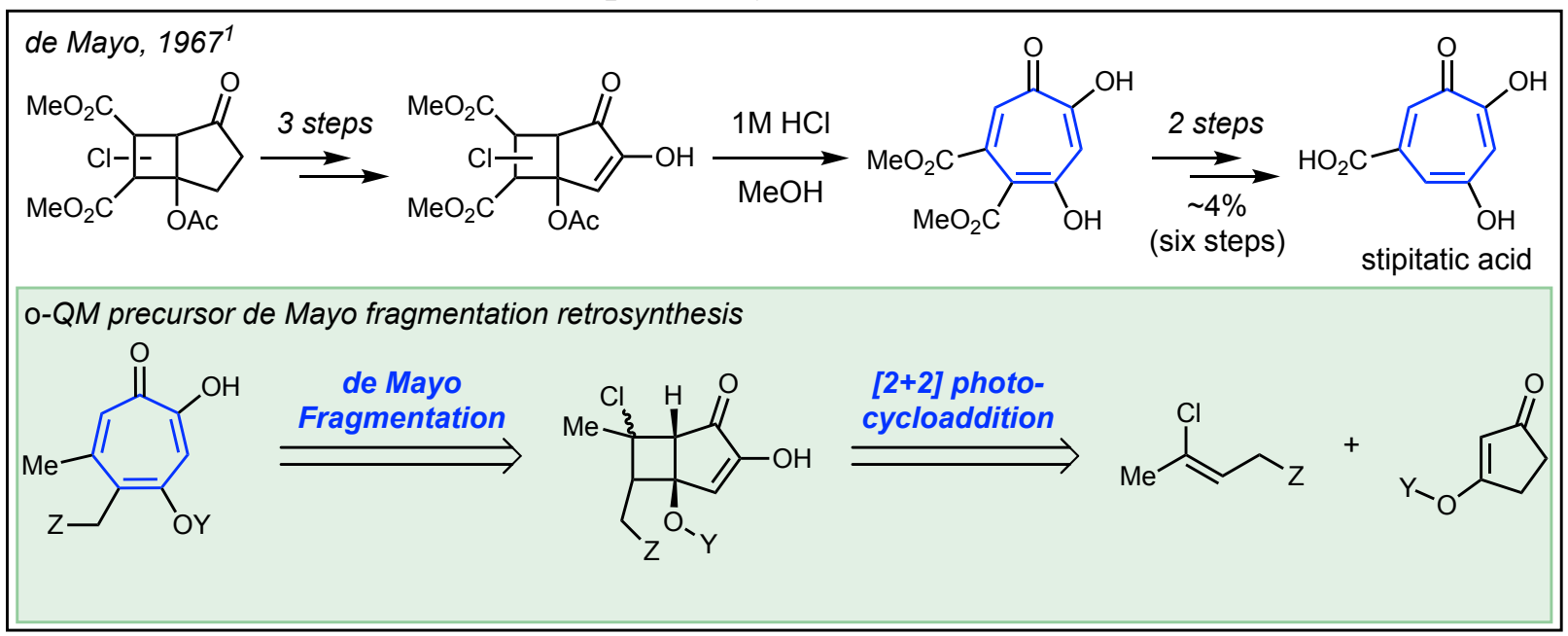

Figure S1. Inspiration and retrosynthetic analysis of tropolone fragment via de Mayo approach. ${ }^{1}$

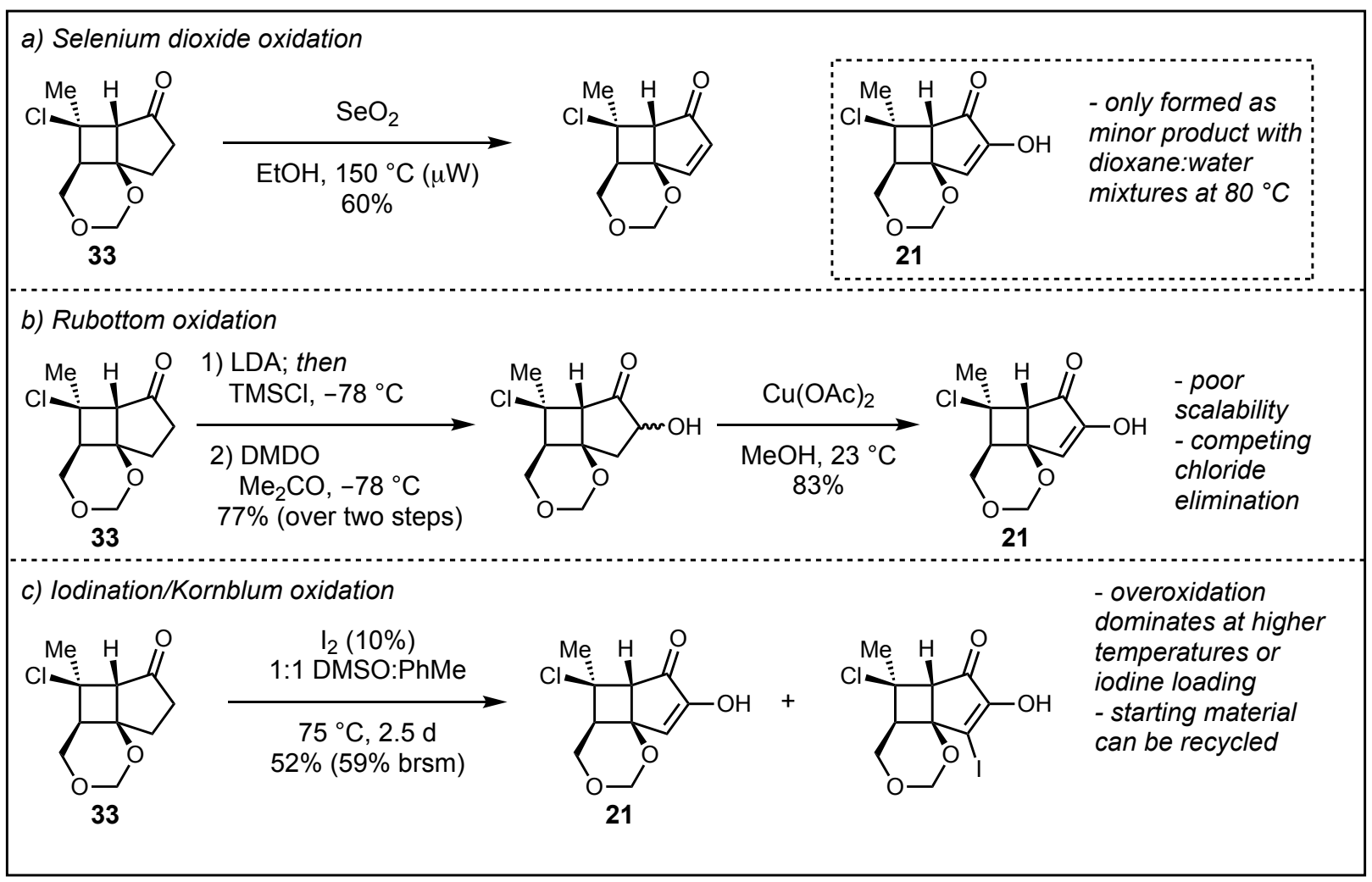

Figure S2. Development of the synthesis of hydroxyenone intermediate $\mathbf{2 1}$. 


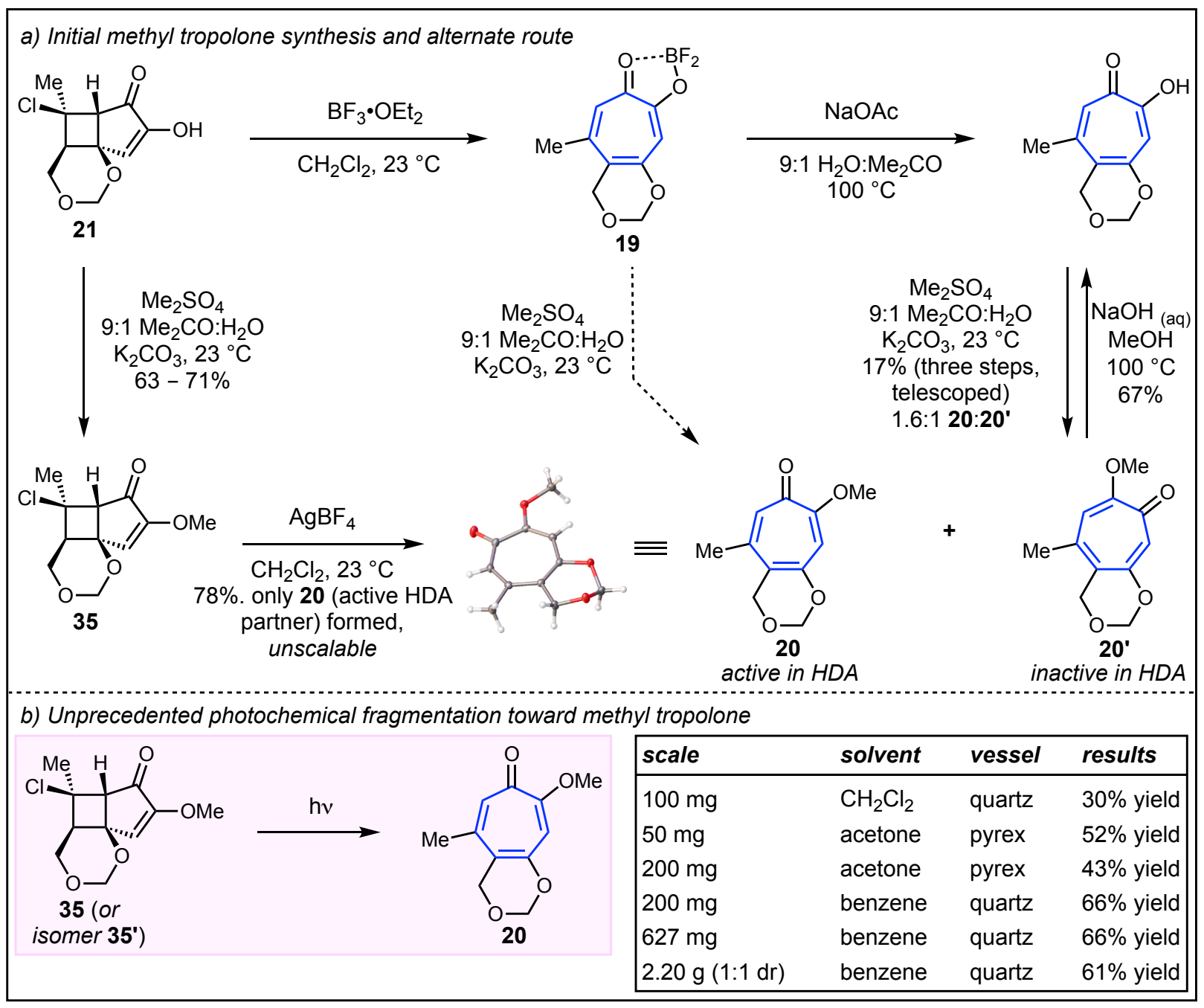

Figure S3. Development of improved methyl tropolone synthesis. 
Macrocycle Synthetic Studies

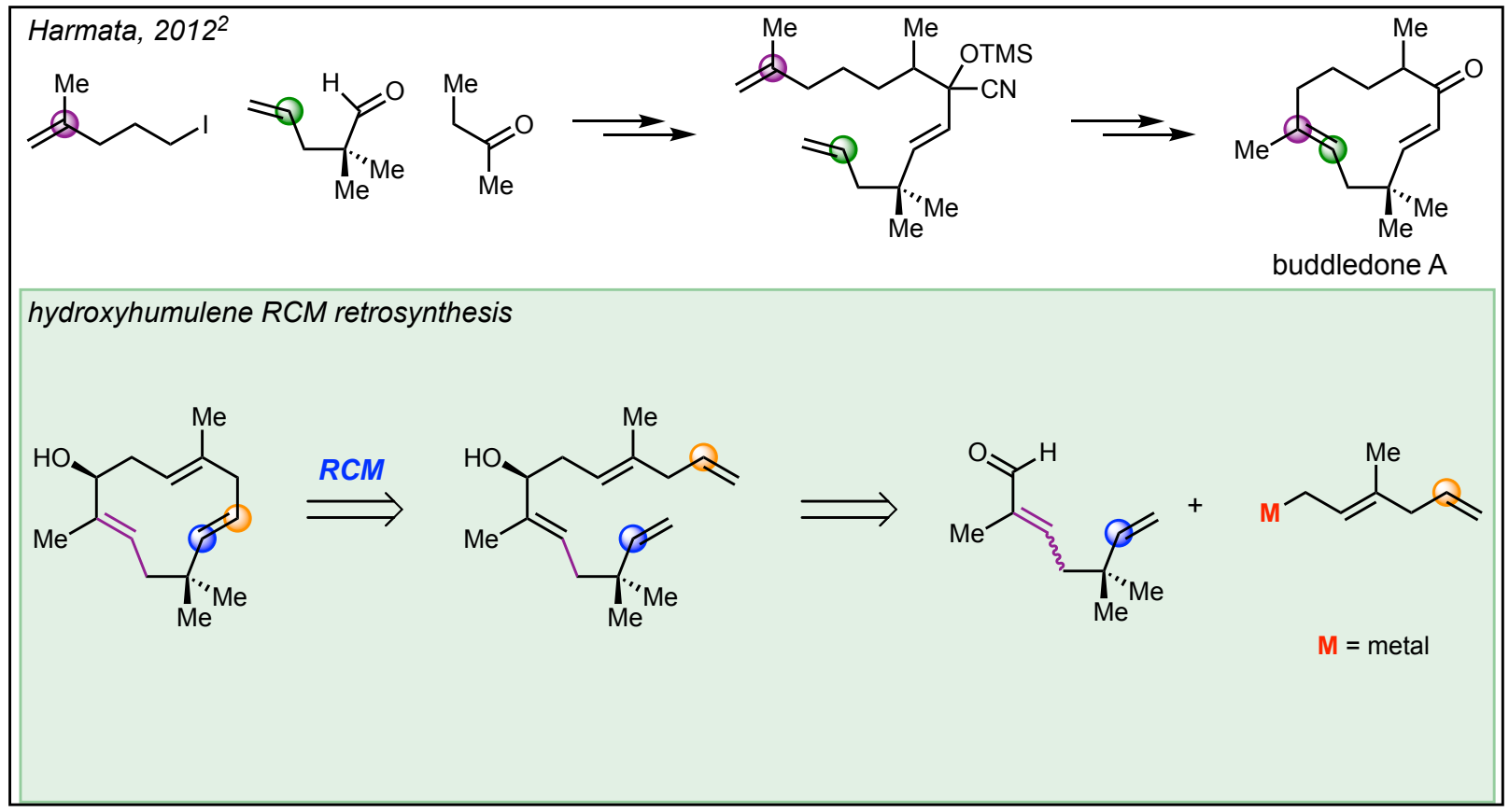

Figure S4. Inspiration and retrosynthetic analysis of sesquiterpene fragment via RCM. ${ }^{2}$

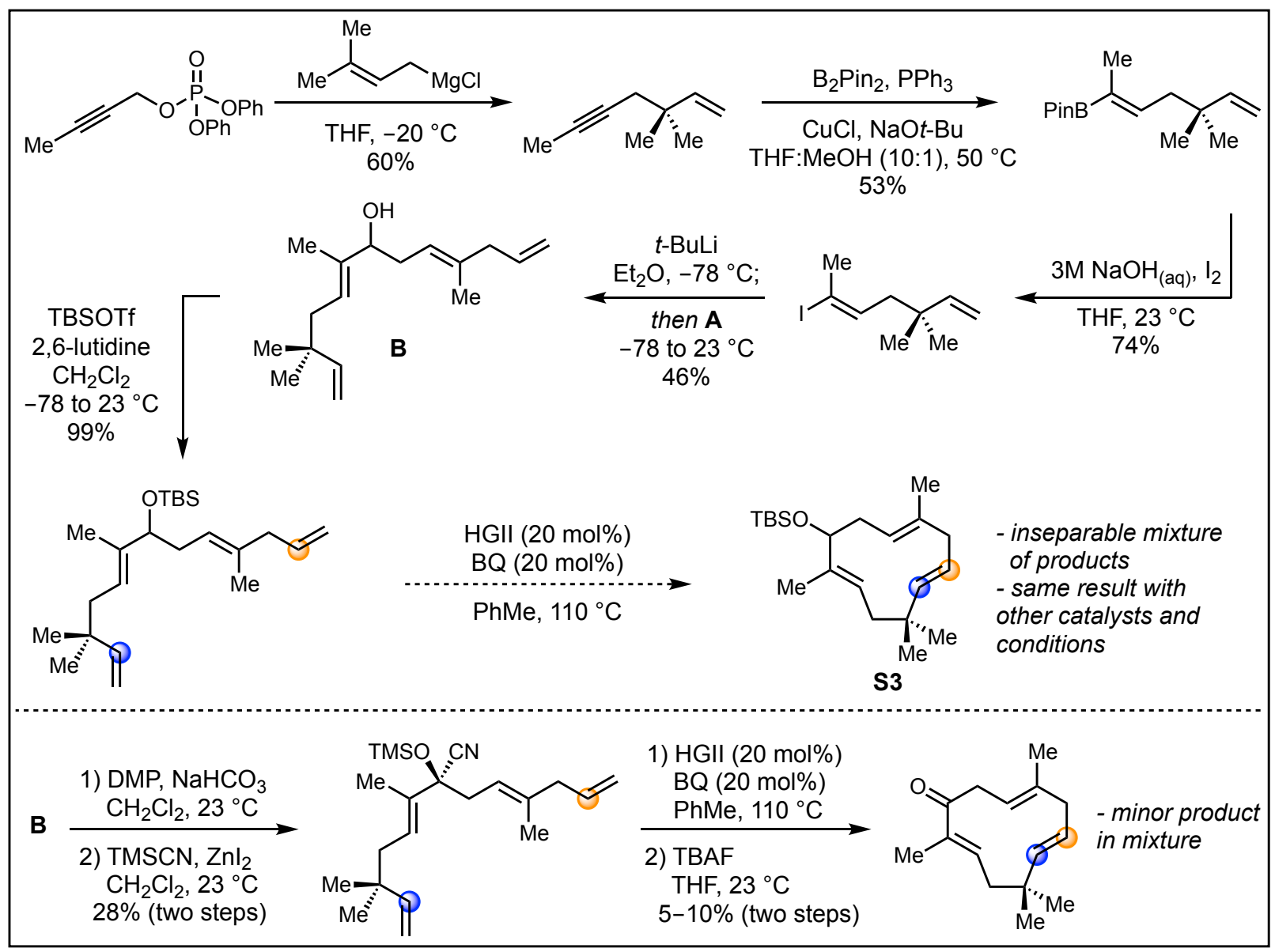

Figure S5. Attempted synthesis of the pycnidione series core by RCM approach. 


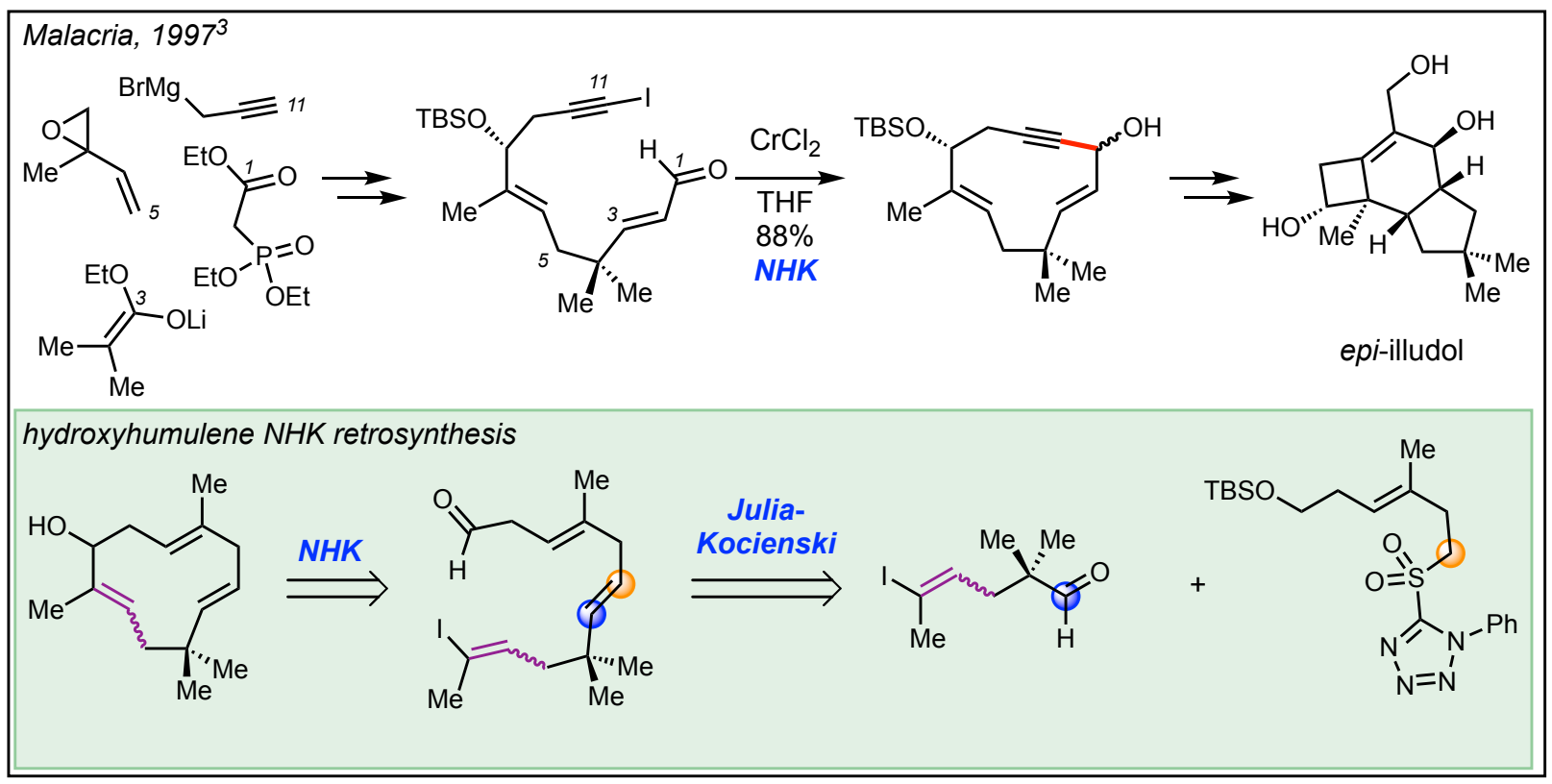

Figure S6. Inspiration and retrosynthetic analysis of humulene cores via NHK approach. ${ }^{3}$ 


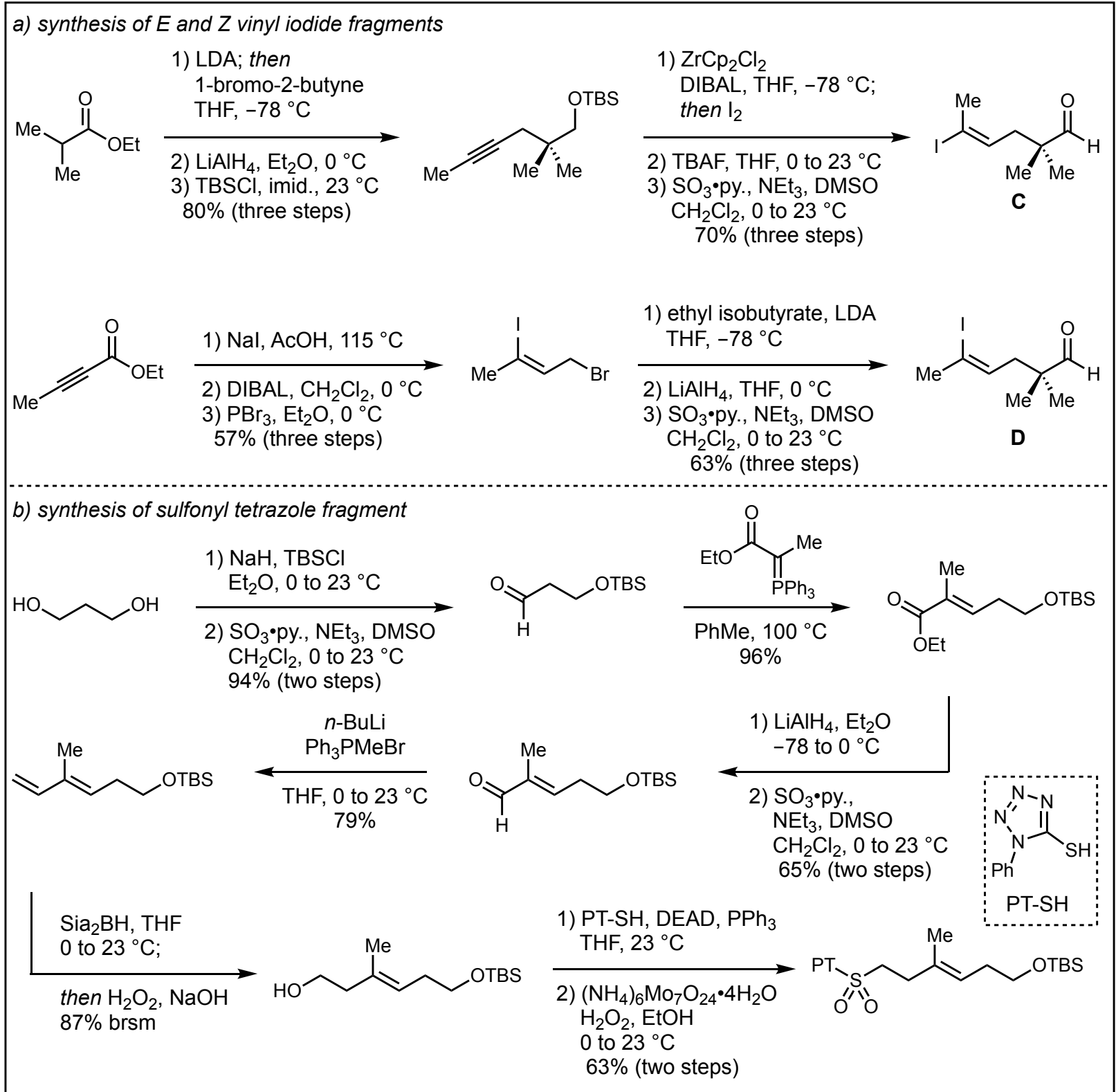

Figure S7. Synthesis of fragments toward macrocycle precursors for synthesis via NHK reaction. 


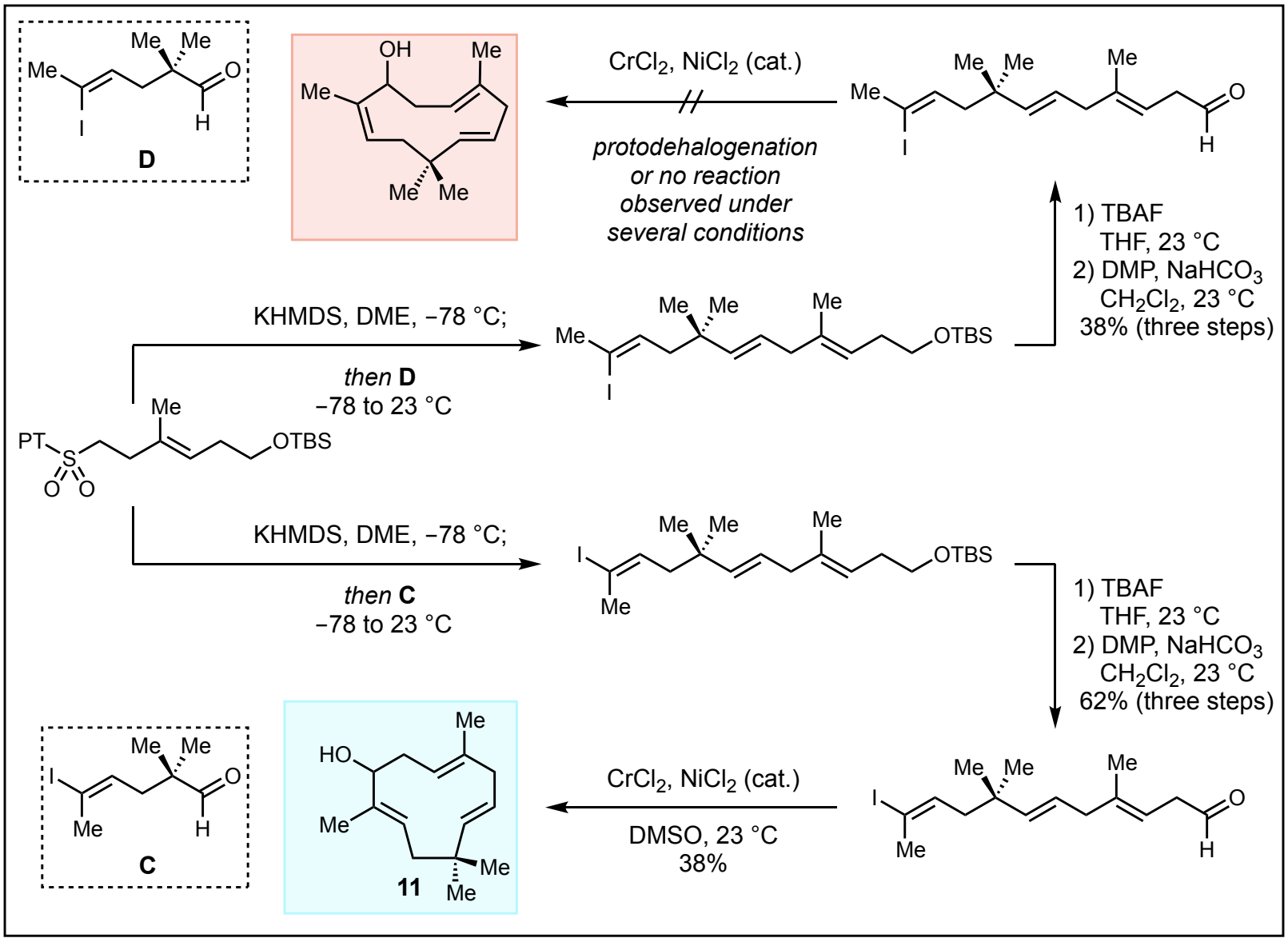

Figure S8. Synthesis macrocycles via the NHK reaction. 


\section{Cycloaddition Synthetic Studies}

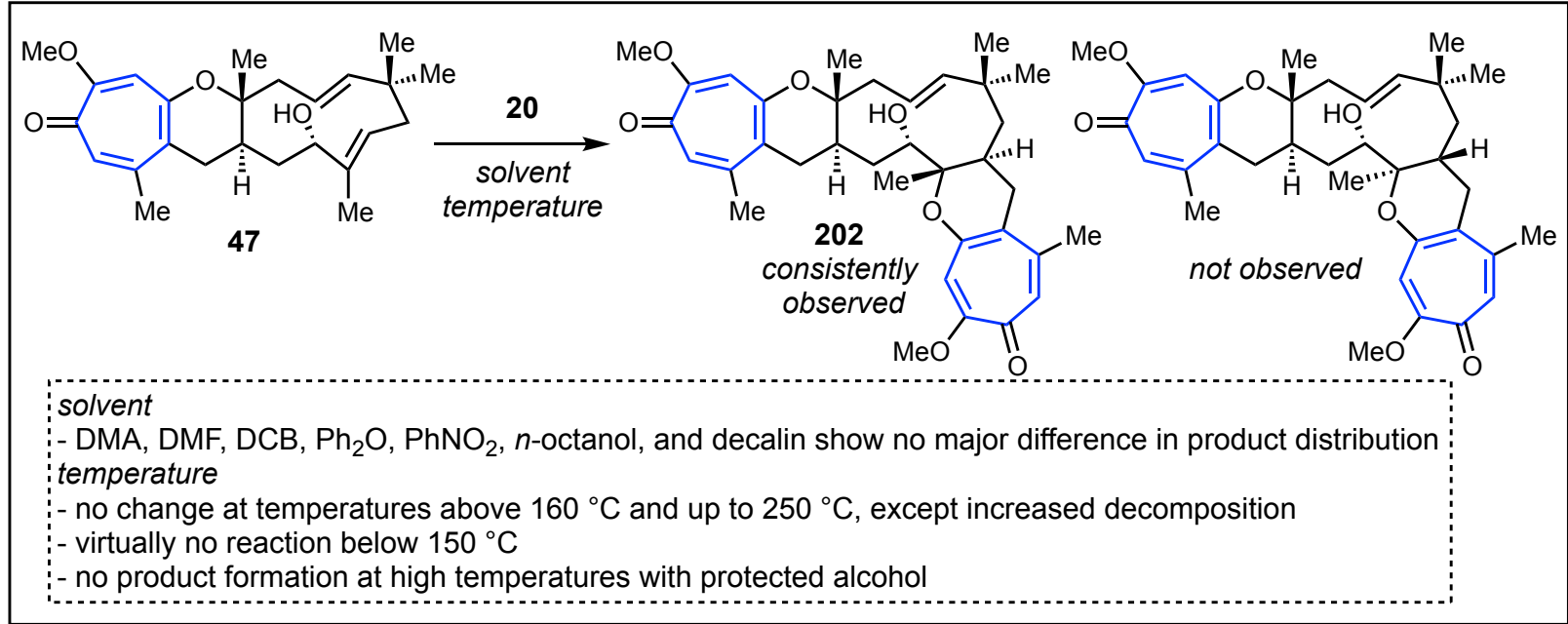

Figure S9. Attempts to change diastereoselectivity of the HDA reaction toward pycnidione.

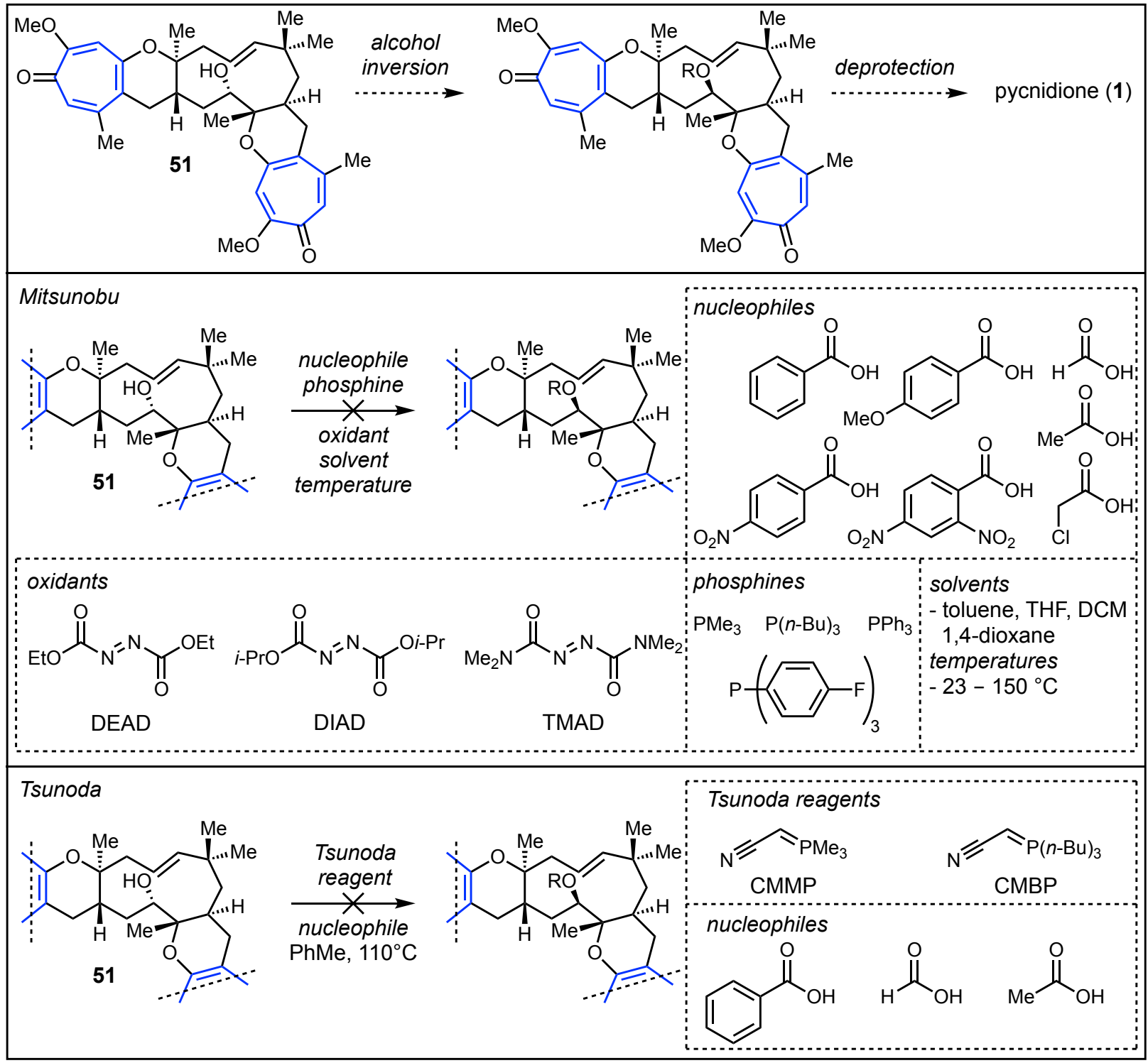

Figure S10. Attempts to invert the secondary alcohol directly en route to pycnidione (1). ${ }^{4}$ 


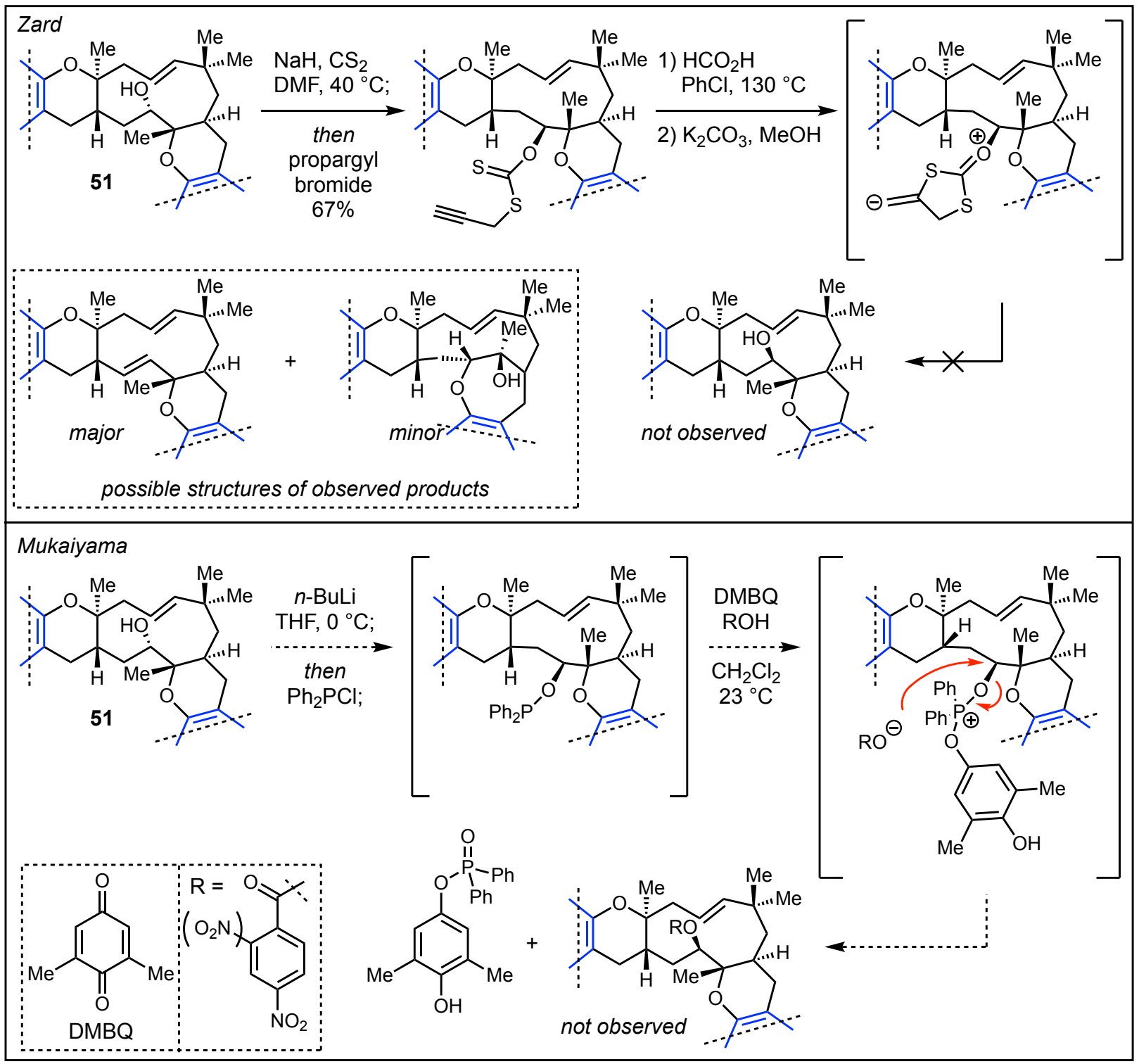

Figure S11. Attempts to invert the secondary alcohol via two step inversion en route to pycnidione (1). ${ }^{5}$

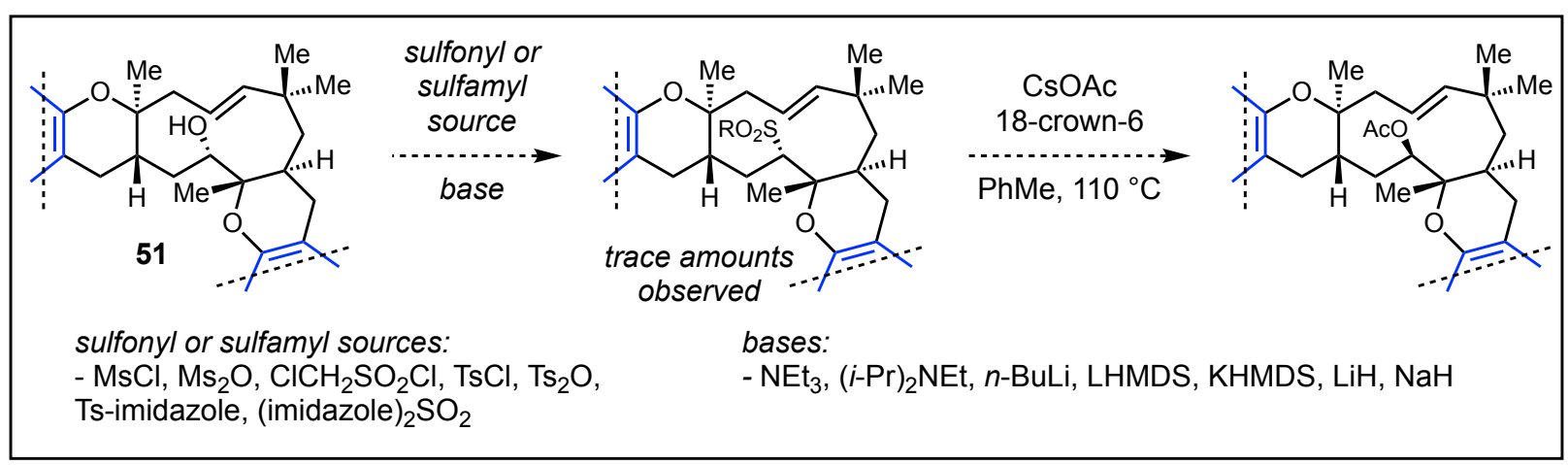

Figure S12. Attempts to activate the secondary alcohol as a sulfonate or sulfamate intended for nucleophilic displacement. ${ }^{6}$ 


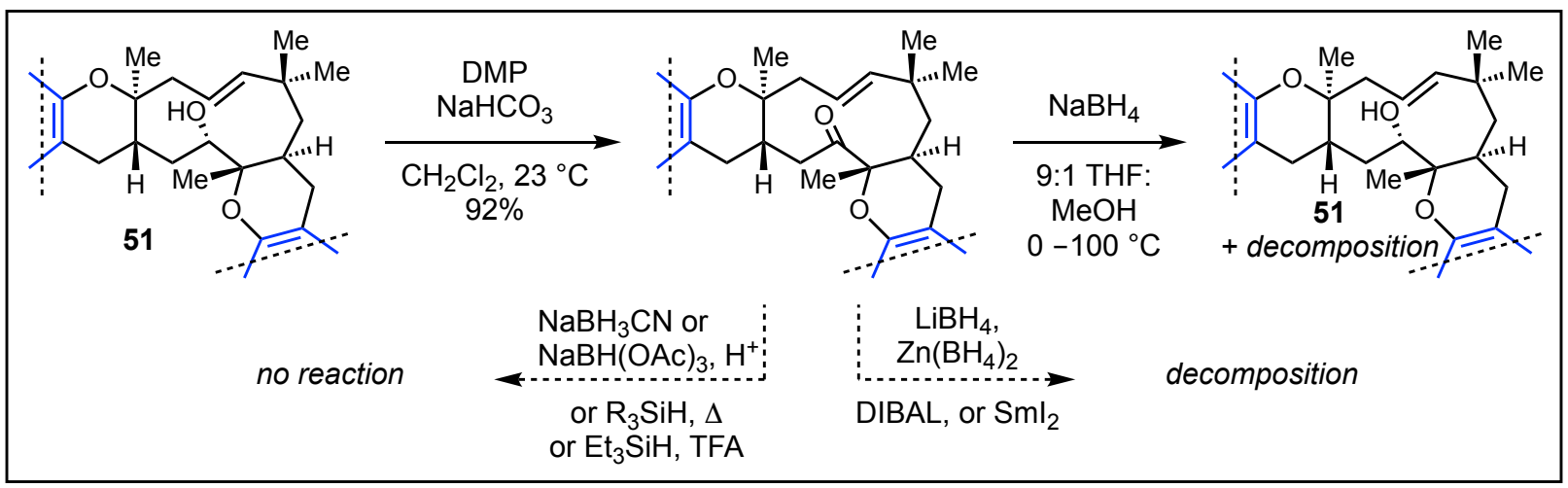

Figure S13. Attempts to reduce the ketone to the alcohol with the desired configuration.

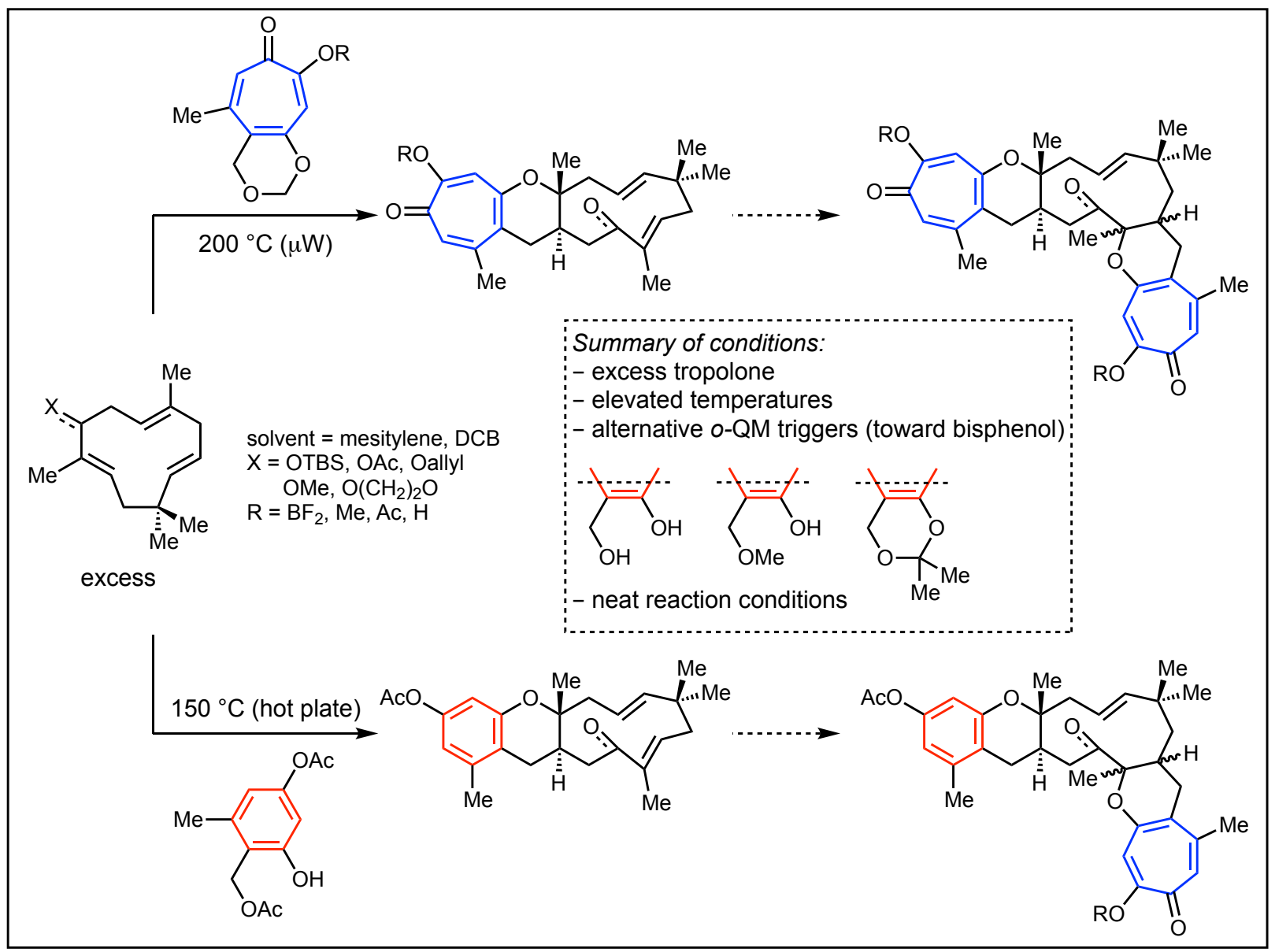

Figure S14. Attempts to reduce the ketone to the alcohol with the desired configuration. 


\section{Experimental Procedures and Characterization Data}

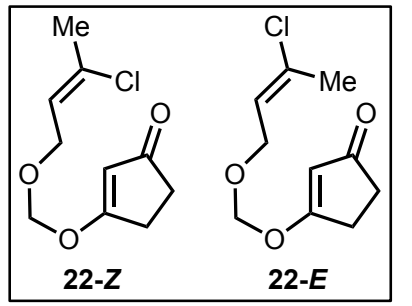

Synthesis of Vinyl Chlorides 22- $Z$ and 22-E: Procedure adapted from reference 4 . $^{7}$ 3-Chloro-2-buten-1-ol (32, $10.7 \mathrm{~g}, 100$ mmol, 1.1 equiv, mixture of $E$ and $Z$ olefin isomers $)^{8}$ was added dropwise to a stirring suspension of paraformaldehyde $(3.00 \mathrm{~g}, 100 \mathrm{mmol}, 1.1$ equiv. $)$ and $\mathrm{TMSCl}(30 \mathrm{~mL})$ at $0{ }^{\circ} \mathrm{C}$.

The flask was equipped with a drying tube containing anhydrous $\mathrm{CaCl}_{2}$, then the reaction was warmed to room temperature and became homogenous over the course of two hours. The reaction was carefully concentrated in vacuo at $10{ }^{\circ} \mathrm{C}$, then the mixture of the newly formed chloromethyl ether and HMDS byproduct was added slowly to a stirring solution of 1,3-cyclopentanedione (31, $8.92 \mathrm{~g}, 91.0 \mathrm{mmol}, 1.0$ equiv.) and potassium carbonate (18.9 g, $136 \mathrm{mmol}, 1.5$ equiv.) in $\mathrm{DMF}(400 \mathrm{~mL})$ at $0{ }^{\circ} \mathrm{C}$. The reaction was warmed to room temperature and stirred for $12 \mathrm{~h}$. Thereafter, the suspension was filtered through a packed bed of celite and vacuum filtered. The reaction flask and filter cake were washed with ethyl acetate $(100 \mathrm{~mL})$ then the orange filtrate was concentrated to $c a .50 \mathrm{~mL}$ by rotary evaporation. The residue was partitioned between water $(50 \mathrm{~mL})$ and ethyl acetate $(50 \mathrm{~mL})$ then the organic and aqueous layers were separated. The aqueous layer was extracted with ethyl acetate $(2 \times 50 \mathrm{~mL})$ then the combined organic extracts were washed with brine $(50 \mathrm{~mL})$, dried over magnesium sulfate, and concentrated. The crude reaction mixture was purified by flash column chromatography (silica gel, 1:1 hexane:EtOAc) to afford an inconsequential mixture of compounds $\mathbf{2 2}-\boldsymbol{Z}$ and $\mathbf{2 2 -} \boldsymbol{E}$ as a pale yellow oil (17.6 g, $81.1 \mathrm{mmol}, 89 \%)$. 22- $\boldsymbol{Z}$ could be completely separated from 22- $\boldsymbol{E}$ by column chromatography. However, 22- $\boldsymbol{E}$ could not be similarly obtained without impurities from $\mathbf{2 2}-\boldsymbol{Z}$. Thus, characterization of 22- $\boldsymbol{E}$ was less extensive.

22- $Z$

$\mathbf{R}_{\mathbf{f}}$

${ }^{1} \mathbf{H}$ NMR $\quad\left(500 \mathrm{MHz}, \mathrm{CDCl}_{3}\right) \delta 5.62(\mathrm{tq}, J=6.3,1.3 \mathrm{~Hz}, 1 \mathrm{H}), 5.43(\mathrm{t}, J=1.3 \mathrm{~Hz}, 1 \mathrm{H}), 5.16(\mathrm{~s}, 2 \mathrm{H})$, $4.32(\mathrm{dq}, J=6.3,1.3 \mathrm{~Hz}, 2 \mathrm{H}), 2.66-2.63(\mathrm{~m}, 2 \mathrm{H}), 2.46-2.43(\mathrm{~m}, 2 \mathrm{H}), 2.14(\mathrm{dt}, J=1.3$ $\mathrm{Hz}, 3 \mathrm{H})$.

${ }^{13}$ C NMR $\quad\left(126 \mathrm{MHz}, \mathrm{CDCl}_{3}\right) \delta 206.2,187.7,134.9,121.3,107.1,94.4,66.6,34.0,28.6,26.4$.

HRMS (ESI-TOF, m/z) calcd. for $\mathrm{C}_{10} \mathrm{H}_{13} \mathrm{ClO}_{3}[\mathrm{M}]^{+}$calc.: 216.0553; found: 216.0551.

IR
(ATR, neat, $\mathrm{cm}^{-1}$ ) 2959 (w), 2924 (w), 1702 (m), 1678 (m), 1589 (s), 1438 (w), 1344 (m), 1287 (w), 1183 (m), 1105 (m), 997 (m), 925 (s), 832 (m), 632 (m), $522(\mathrm{w}), 485(\mathrm{w})$. 
${ }^{1}$ H NMR $\quad\left(500 \mathrm{MHz}, \mathrm{CDCl}_{3}\right) \delta 5.77(\mathrm{tq}, J=7.4,1.3 \mathrm{~Hz}, 1 \mathrm{H}), 5.45(\mathrm{t}, J=1.3 \mathrm{~Hz}, 1 \mathrm{H}), 5.16(\mathrm{~s}, 2 \mathrm{H})$, $4.18(\mathrm{~d}, J=7.4 \mathrm{~Hz}, 2 \mathrm{H}), 2.67-2.63(\mathrm{~m}, 2 \mathrm{H}), 2.47-2.43(\mathrm{~m}, 2 \mathrm{H}), 2.14(\mathrm{~s}, 3 \mathrm{H})$.

Synthesis of Ketones 33 and 33': A mixture of 22 isomers $(4.20 \mathrm{~mL}, 5.00 \mathrm{~g}, 23.1 \mathrm{mmol})$ and

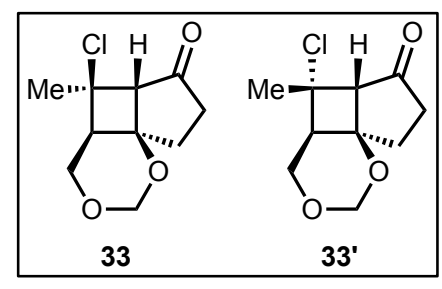

dichloromethane $(600 \mathrm{~mL}, 0.04 \mathrm{M})$

in a 1-L quartz vessel was degassed

with argon at $0{ }^{\circ} \mathrm{C}$ for $20 \mathrm{~min}$.

Thereafter, the solution was

irradiated using six UV lamps for $30 \mathrm{~h}$ (see Picture S1, right). The mixture was concentrated and subjected to flash column chromatography (silica gel, 3:2 hexanes:EtOAc) to give [2+2] cycloadducts $\mathbf{3 3}$ and $\mathbf{3 3}^{\prime}$ as a pale yellow solid (3.70 g, $17.1 \mathrm{mmol}$, $74 \%, d . r .=5.0: 1)$. The diastereomers were partially separable by column chromatography. Further purification of compound $\mathbf{3 3}$ or $\mathbf{3 3}^{\prime}$ could be achieved by recrystallization from dichloromethane

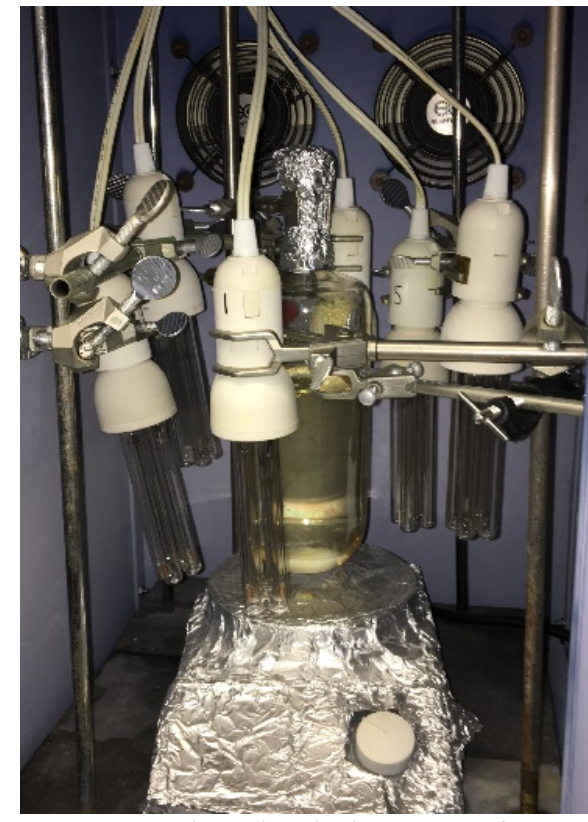

Picture S1. Photochemical apparatus for large scale, batch reactions.

and diethyl ether. Alternatively, the crude reaction mixture could be concentrated to give a tan solid mixture of $\mathbf{3 3}$ and $\mathbf{3 3}^{\prime}$. Suspension of this residue in diethyl ether and filtration of the solid though a fritted funnel gave $\mathbf{3 3}$ with only trace quantities of $\mathbf{3 3}^{\prime}$ as a colorless solid.

33

$\mathbf{R}_{\mathbf{f}} \quad 0.3\left(\mathrm{SiO}_{2}\right.$, hexanes:EtOAc $\left.=3: 2, \mathrm{KMnO}_{4}\right)$.

${ }^{1}$ H NMR $\quad\left(500 \mathrm{MHz}, \mathrm{CD}_{2} \mathrm{Cl}_{2}\right) \delta 4.97(\mathrm{~d}, J=6.3 \mathrm{~Hz}, 1 \mathrm{H}), 4.70(\mathrm{~d}, J=6.3 \mathrm{~Hz}, 1 \mathrm{H}), 4.23(\mathrm{~d}, J=12.8$ $\mathrm{Hz}, 1 \mathrm{H}), 3.99(\mathrm{dd}, J=12.8,6.3 \mathrm{~Hz}, 1 \mathrm{H}), 3.01(\mathrm{~s}, 1 \mathrm{H}), 2.63-2.52(\mathrm{~m}, 1 \mathrm{H}), 2.46-2.35(\mathrm{~m}$, $3 \mathrm{H}), 2.02-1.91(\mathrm{~m}, 1 \mathrm{H}), 1.52(\mathrm{~s}, 3 \mathrm{H})$.

${ }^{13}$ C NMR $\quad\left(126 \mathrm{MHz}, \mathrm{CD}_{2} \mathrm{Cl}_{2}\right) \delta 213.0,89.5,77.5,65.7,63.9,63.7,46.4,39.3,32.6,29.2$.

HRMS (EI-TOF, m/z) calcd. for $\mathrm{C}_{10} \mathrm{H}_{13} \mathrm{ClO}_{3}[\mathrm{M}]^{+}$calc.: 216.0553; found: 216.0555.

IR

(ATR, neat, $\left.\mathrm{cm}^{-1}\right) 3448(\mathrm{w}), 2985(\mathrm{w}), 2941(\mathrm{w}), 2859(\mathrm{w}), 2787(\mathrm{w}), 1732(\mathrm{~s}), 1381(\mathrm{w})$, 1334 (w), 1156 (s), 1009 (s), $914(\mathrm{~m}), 795$ (w), $760(\mathrm{w}), 646(\mathrm{w}), 602(\mathrm{w}), 512(\mathrm{~m}), 461$ (m).

$\quad \mathbf{m p} \quad 133-136^{\circ} \mathrm{C}$ 
$\mathbf{R}_{\mathbf{f}} \quad 0.5\left(\mathrm{SiO}_{2}\right.$, hexanes:EtOAc $\left.=3: 2, \mathrm{KMnO}_{4}\right)$.

${ }^{1}$ H NMR $\quad\left(500 \mathrm{MHz}, \mathrm{CD}_{2} \mathrm{Cl}_{2}\right) \delta 4.92(\mathrm{~d}, J=6.6 \mathrm{~Hz}, 1 \mathrm{H}), 4.61(\mathrm{~d}, J=6.5 \mathrm{~Hz}, 1 \mathrm{H}), 4.26(\mathrm{~d}, J=13.2$ $\mathrm{Hz}, 1 \mathrm{H}), 3.91(\mathrm{dd}, J=13.2,5.7 \mathrm{~Hz}, 1 \mathrm{H}), 2.69(\mathrm{~s}, 1 \mathrm{H}), 2.67$ (d, $5.7 \mathrm{~Hz}, 1 \mathrm{H}), 2.58-2.49$ (m, $2 \mathrm{H}), 2.45$ (ddd, 12.7, 7.4, 2.6, 1H), $2.02(\mathrm{~s}, 3 \mathrm{H}), 1.88(\mathrm{td}, 11.7,9.5 \mathrm{1H})$.

${ }^{13} \mathrm{C} \mathrm{NMR} \quad\left(126 \mathrm{MHz}, \mathrm{CD}_{2} \mathrm{Cl}_{2}\right) \delta 211.1,89.7,77.6,64.1,62.6,62.5,47.7,39.2,31.8,28.1$.

HRMS (EI-TOF, m/z) calcd. for $\mathrm{C}_{10} \mathrm{H}_{13} \mathrm{ClO}_{3}[\mathrm{M}]^{+}$calc.: 216.0553; found: 216.0547.

IR

(ATR, neat, $\left.\mathrm{cm}^{-1}\right) 2977(\mathrm{w}), 2881(\mathrm{w}), 1724(\mathrm{~s}), 1491(\mathrm{w}), 1458(\mathrm{w}), 1412(\mathrm{w}), 1384(\mathrm{~m})$, $1216(\mathrm{~m}), 1161$ (s), 1006 (s), 963 (s), 801 (m), 737 (m), 643 (m), 456 (m).

mp $\quad 142-143^{\circ} \mathrm{C}$.

Synthesis of Hydroxyenone 21: To a solution of compound 33 (2.50 g, 11.5 mmol, 1.0 equiv.) in a 1:1

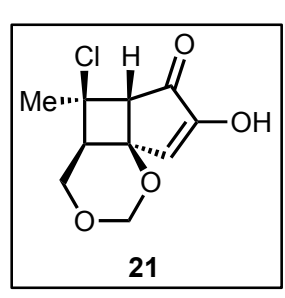
mixture of DMSO and PhMe (120 mL, 0.1 M) was added iodine (0.293 g, $1.15 \mathrm{mmol}$, 0.10 equiv.). A condenser was attached, and the dark red solution was stirred at $75^{\circ} \mathrm{C}$ in the absence of light and the consumption of the starting material was monitored by crude NMR. After 2.5 days, the reaction was stopped before full consumption of the starting material to mitigate over-oxidation of the desired product. The solution was cooled to room temperature and quenched with aqueous $10 \%$ sodium thiosulfate $(75 \mathrm{~mL})$. The mixture was extracted with ethyl acetate $(4 \times 50 \mathrm{~mL})$ then the combined organic extracts were washed with water $(3 \times 50 \mathrm{~mL})$, brine $(50 \mathrm{~mL})$, dried with magnesium sulfate, filtered, and concentrated under reduced pressure. The brown residue was subjected to column chromatography (silica gel, 3:2 to 1:2 hexanes: $\mathrm{Et}_{2} \mathrm{O}$ gradient) to give recovered $33(0.287 \mathrm{~g}, 1.32 \mathrm{mmol})$ and compound 21 as a pale yellow solid (1.384 g, $6.00 \mathrm{mmol}, 52 \%$, $59 \%$ brsm).

$\mathbf{R}_{\mathbf{f}} \quad 0.4\left(\mathrm{SiO}_{2}\right.$, hexanes:EtOAc $\left.=1: 1, \mathrm{UV}, \mathrm{KMnO}_{4}\right)$.

${ }^{1} \mathbf{H}$ NMR $\quad\left(500 \mathrm{MHz}, \mathrm{CDCl}_{3}\right) \delta 6.47(\mathrm{~d}, J=0.7 \mathrm{~Hz}, 1 \mathrm{H}), 5.67(\mathrm{~s}, 1 \mathrm{H}), 5.10(\mathrm{~d}, J=5.6 \mathrm{~Hz}, 1 \mathrm{H}), 4.89$ $(\mathrm{d}, J=5.6 \mathrm{~Hz}, 1 \mathrm{H}), 4.45$ (ddd, $J=12.4,2.5 \mathrm{~Hz}, 1 \mathrm{H}), 4.12$ (dd, $J=12.4,7.0 \mathrm{~Hz}, 1 \mathrm{H}), 3.49$ (dd, $J=1.2,0.7 \mathrm{~Hz}, 1 \mathrm{H}), 2.48(\mathrm{ddd}, J=7.0,2.5,1.2 \mathrm{~Hz}, 1 \mathrm{H}), 1.54$ (s, 3H).

${ }^{13}$ C NMR $\quad\left(126 \mathrm{MHz}, \mathrm{CDCl}_{3}\right) \delta 197.5,154.9,127.2,90.6,71.6,64.5,62.6,61.2,46.5,29.1$.

HRMS (EI-TOF, m/z) calcd. for $\mathrm{C}_{10} \mathrm{H}_{11} \mathrm{ClO}_{4}[\mathrm{M}]^{+}$calc.: 230.0346; found: 230.0341.

IR

(ATR, neat, $\left.\mathrm{cm}^{-1}\right) 3284$ (br), 3124 (br), 3010 (w), 2859 (w), 1713 (s), 1646 (m, 1387 (m), $1231(\mathrm{w}), 1208$ (m), 1117 (s), 1033 (s), 990 (s), 963 (m), 801 (m), $683(\mathrm{~m}), 549(\mathrm{~m}), 485$ (m). 
Synthesis of Hydroxyenone 21': Employing a procedure identical to that for the formation of compound

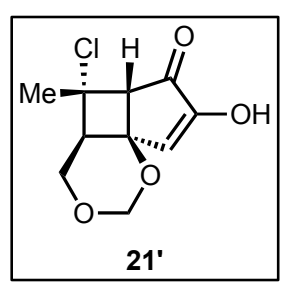
21 from 33, compound $\mathbf{3 3}^{\prime}(2.50 \mathrm{~g}, 11.5 \mathrm{mmol}, 1.0$ equiv. $)$ in a $1: 1$ mixture of DMSO and $\mathrm{PhMe}(120 \mathrm{~mL}, 0.1 \mathrm{M})$ was treated with iodine (0.293 g, $1.15 \mathrm{mmol}, 0.10$ equiv.), heated at $75{ }^{\circ} \mathrm{C}$ for 2.5 days, and monitored by TLC (silica gel, 1:1 hexanes:EtOAc). Following work-up, the crude product mixture was purified by column chromatography (silica gel, 4:1 to 1:1 hexanes:EtOAc gradient) to produce recovered $\mathbf{3 3}^{\prime}(0.947 \mathrm{~g}, 4.37 \mathrm{mmol})$ and compound $\mathbf{2 1}^{\prime}$ as a pale yellow solid $(0.741 \mathrm{~g}, 3.21 \mathrm{mmol}, 28 \%, 45 \% \mathrm{brsm})$.

$\mathbf{R}_{\mathbf{f}}$ $0.3\left(\mathrm{SiO}_{2}\right.$, hexanes:EtOAc $\left.=3: 1, \mathrm{UV}, \mathrm{KMnO}_{4}\right)$.

${ }^{1}$ H NMR $\quad\left(500 \mathrm{MHz}, \mathrm{CDCl}_{3}\right) \delta 6.64(\mathrm{~s}, 1 \mathrm{H}), 5.92(\mathrm{~s}, 1 \mathrm{H}), 5.02(\mathrm{~d}, J=6.1 \mathrm{~Hz}, 1 \mathrm{H}), 4.73(\mathrm{~d}, J=6.1$ $\mathrm{Hz}, 1 \mathrm{H}), 4.29(\mathrm{~d}, J=13.0 \mathrm{~Hz}, 1 \mathrm{H}), 3.99(\mathrm{dd}, J=13.0,6.1 \mathrm{~Hz}, 1 \mathrm{H}), 3.06(\mathrm{~d}, J=1.2 \mathrm{~Hz}$, $1 \mathrm{H}), 2.65(\mathrm{~d}, J=6.1 \mathrm{~Hz}, 1 \mathrm{H}), 2.04(\mathrm{~s}, 3 \mathrm{H})$.

${ }^{13} \mathrm{C} \mathrm{NMR} \quad\left(126 \mathrm{MHz}, \mathrm{CDCl}_{3}\right) \delta 196.4,155.2,128.1,90.3,72.9,62.9,60.0,59.6,50.0,28.7$.

HRMS (EI-TOF, m/z) calcd. for $\mathrm{C}_{10} \mathrm{H}_{11} \mathrm{ClO}_{4}[\mathrm{M}]^{+}$calc.: 230.0346; found: 230.0341.

IR

(ATR, neat, $\left.\mathrm{cm}^{-1}\right) 3341(\mathrm{br}), 2974(\mathrm{w}), 2872(\mathrm{w}), 1706(\mathrm{~m}), 1644(\mathrm{~m}), 1396(\mathrm{~m}), 1231(\mathrm{~m})$, 1171 (m), 1115 (s), 1131 (s), 986 (s), 921 (m), 799 (m), 701 (m), 644 (m), 610 (m), 571 (m).

mp $\quad 142-145^{\circ} \mathrm{C}$.

Synthesis of Methoxy-enol Ether 35: To a solution of compound 21 (3.65 g, 15.8 mmol, 1.0 equiv.) and

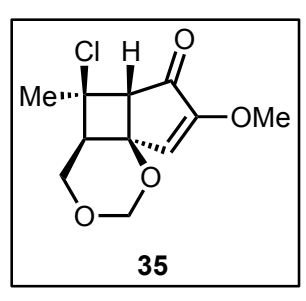
potassium carbonate ( $8.75 \mathrm{~g}, 63.3 \mathrm{mmol}, 4.0$ equiv.) in a 9:1 acetone:water mixture (100 mL) under nitrogen was added $\mathrm{Me}_{2} \mathrm{SO}_{4}$ (4.50 mL, $47.5 \mathrm{mmol}, 3.0$ equiv.) dropwise. The reaction started as a suspension and gradually became a yellow solution over 1.5 hours. After stirring for 24 hours, the reaction was checked by TLC (1:1 hexanes:EtOAc) and appeared to be nearly complete. Brine $(50 \mathrm{~mL})$ was added to the reaction, and the mixture was transferred to a separatory funnel. The aqueous layer was extracted with ethyl acetate $(3 \times 50$ $\mathrm{mL})$, then the combined organic extracts were again washed with brine $(50 \mathrm{~mL})$, dried over magnesium sulfate, filtered, and concentrated under reduced pressure. The crude product was purified by column 
chromatography (silica gel, 9:1 to 1:1 hexanes:EtOAc) to give recovered $\mathbf{2 1}(0.528 \mathrm{~g}, 2.29 \mathrm{mmol})$ and $\mathbf{3 5}$ (2.239 g, $9.15 \mathrm{mmol}, 58 \%, 68 \%$ brsm) as pale solids.

$\mathbf{R}_{\mathbf{f}} \quad 0.3\left(\mathrm{SiO}_{2}\right.$, hexanes:EtOAc $\left.=1: 1, \mathrm{UV}, \mathrm{KMnO}_{4}\right)$.

${ }^{1}$ H NMR $\quad\left(500 \mathrm{MHz}, \mathrm{CDCl}_{3}\right) \delta 6.31(\mathrm{~s}, 1 \mathrm{H}), 5.03(\mathrm{~d}, J=5.7 \mathrm{~Hz}, 1 \mathrm{H}), 4.83(\mathrm{~d}, J=5.7 \mathrm{~Hz}, 1 \mathrm{H}), 4.37$ (ddd, $J=12.5,2.5,1.1,1 \mathrm{H}), 4.07(\mathrm{dd}, J=12.5,7.1 \mathrm{~Hz}, 1 \mathrm{H}), 3.72(\mathrm{~s}, 3 \mathrm{H}), 3.38(\mathrm{~s}, 1 \mathrm{H})$, 2.43 (ddd, $J=7.1,2.5,1.1 \mathrm{~Hz}, 1 \mathrm{H}), 1.50(\mathrm{~s}, 3 \mathrm{H})$.

${ }^{13}$ C NMR $\quad\left(126 \mathrm{MHz}, \mathrm{CDCl}_{3}\right) \delta 195.6,159.4,125.2,90.3,71.2,64.3,62.8,61.8,57.7,46.7,29.0$.

HRMS (ESI-TOF, m/z) calcd. for $\mathrm{C}_{11} \mathrm{H}_{14} \mathrm{ClO}_{4}[\mathrm{M}+\mathrm{H}]^{+}$calc.: 245.0581; found: 245.0570.

IR (ATR, neat, $\left.\mathrm{cm}^{-1}\right) 3078(\mathrm{w}), 2975(\mathrm{w}), 2944(\mathrm{w}), 2854(\mathrm{w}), 1711(\mathrm{~s}), 1617(\mathrm{~s}), 1439(\mathrm{~m})$, 1368 (m), 1290 (m), 1208 (m), 1177 (m), 1123 (s), 1043 (s), 974 (m), 929 (m), 690 (m), $599(\mathrm{~m}), 501(\mathrm{~m})$.

mp $\quad 90-91^{\circ} \mathrm{C}$.

Synthesis of Methoxy-enol Ether $\mathbf{3 5}^{\prime}$ : Employing a procedure identical to that used for the conversion of

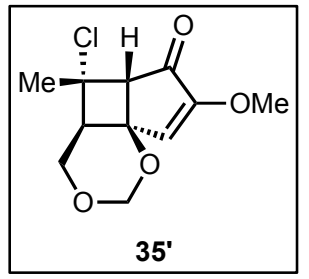

21 to 35 and subsequent purification, 21' (0.543 g, $2.40 \mathrm{mmol}, 1.0$ equiv.) was methylated with potassium carbonate $(1.50 \mathrm{~g}, 14.0 \mathrm{mmol}, 6.0$ equiv.) and dimethylsulfate $(1.10 \mathrm{~mL}, 12.0 \mathrm{mmol}, 5.0$ equiv. $)$ in 9:1 acetone:water $(30 \mathrm{~mL})$ to give 35' (0.432 g, $1.77 \mathrm{mmol}, 74 \%)$.

$\mathbf{R}_{\mathbf{f}}$ $0.4\left(\mathrm{SiO}_{2}\right.$, hexanes:EtOAc $\left.=1: 1, \mathrm{UV}, \mathrm{KMnO}_{4}\right)$.

${ }^{1}$ H NMR $\quad\left(500 \mathrm{MHz}, \mathrm{CDCl}_{3}\right) \delta 6.48(\mathrm{~s}, 1 \mathrm{H}), 5.02(\mathrm{dd}, J=6.1,1.1 \mathrm{~Hz}, 1 \mathrm{H}), 4.75(\mathrm{~d}, J=6.1 \mathrm{~Hz}, 1 \mathrm{H})$, $4.29(\mathrm{dd}, J=13.0,1.1 \mathrm{~Hz}, 1 \mathrm{H}), 3.99(\mathrm{dd}, J=13.0,6.1 \mathrm{~Hz}, 1 \mathrm{H}), 3.78(\mathrm{~s}, 3 \mathrm{H}), 3.04(\mathrm{~s}, 1 \mathrm{H})$, $2.66(\mathrm{~d}, J=6.1 \mathrm{~Hz}, 1 \mathrm{H}), 2.02(\mathrm{~s}, 3 \mathrm{H})$.

${ }^{13} \mathbf{C ~ N M R ~} \quad\left(126 \mathrm{MHz}, \mathrm{CDCl}_{3}\right) \delta{ }^{13} \mathrm{C} \mathrm{NMR}\left(126 \mathrm{MHz}, \mathrm{CDCl}_{3}\right) \delta 194.5,159.9,125.8,90.3,72.4,62.9$, $60.3,60.0,57.8,50.4,28.7$.

HRMS (ESI-TOF, m/z) calcd. for $\mathrm{C}_{11} \mathrm{H}_{14} \mathrm{ClO}_{4}[\mathrm{M}+\mathrm{H}]^{+}$calc.: 245.0581; found: 245.0570.

IR $\quad\left(A T R\right.$, neat, $\left.\mathrm{cm}^{-1}\right) 3080(\mathrm{w}), 2949(\mathrm{w}), 2867(\mathrm{w}), 1716(\mathrm{~s}), 1617(\mathrm{~s}), 1439(\mathrm{w}), 1318(\mathrm{~m})$, 1234 (m), 1111 (s), 1039 (s), 982 (s), 803 (m), 706 (m), 609 (m).

mp $\quad 143-145^{\circ} \mathrm{C}$.

Synthesis of Tropolone-Adduct 19: To a solution of compound 21 (3.11 g, $13.5 \mathrm{mmol}, 1.0$ equiv) in dichloromethane $(135 \mathrm{~mL}, 0.1 \mathrm{M})$ at $0{ }^{\circ} \mathrm{C}$ was added boron trifluoride diethyl etherate $(10.0 \mathrm{~mL}, 80.8 \mathrm{mmol}$, 6.0 equiv.) dropwise. The reaction was slowly warmed to room temperature and stirred for two days, after 


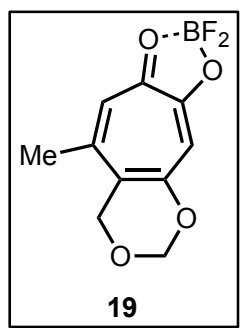

which the starting material was shown to have been consumed by TLC (silica gel, 1:1 hexanes:EtOAc). The reaction was quenched with saturated aqueous sodium bicarbonate $(50 \mathrm{~mL})$ and transferred to a separatory funnel. The dichloromethane layer was drained, and the aqueous layer was extracted with ethyl acetate $(4 \times 50 \mathrm{~mL})$. The combined organic layers were washed with brine $(50 \mathrm{~mL})$, dried over magnesium sulfate, filtered, and concentrated to a brown solid. The residue was purified by column chromatography (silica gel, $\mathrm{CH}_{2} \mathrm{Cl}_{2}$ ) to afford compound 19 as a colorless solid (1.91 g, $7.88 \mathrm{mmol}, 59 \%)$. Compound 21' (0.782 g, $3.39 \mathrm{mmol})$ may be converted to compound 19 (0.356 g, $1.47 \mathrm{mmol}, 43 \%)$ using an analogous procedure.

$\mathbf{R}_{\mathbf{r}}$ $0.3\left(\mathrm{SiO}_{2}\right.$, hexanes:EtOAc $\left.=2: 3, \mathrm{CH}_{2} \mathrm{Cl}_{2}=0.59, \mathrm{UV}, \mathrm{KMnO}_{4}\right)$

${ }^{1}$ H NMR $\quad\left(500 \mathrm{MHz},\left(\mathrm{CD}_{3}\right)_{2} \mathrm{CO}\right) \delta 7.57(\mathrm{~s}, 1 \mathrm{H}), 7.31(\mathrm{~s}, 1 \mathrm{H}), 5.50(\mathrm{~s}, 2 \mathrm{H}), 5.08(\mathrm{~s}, 2 \mathrm{H}), 2.60(\mathrm{~s}, 3 \mathrm{H})$.

${ }^{13}$ C NMR $\quad\left(126 \mathrm{MHz},\left(\mathrm{CD}_{3}\right)_{2} \mathrm{CO}\right) \delta 169.03,168.96168 .0,157.3,128.6,122.5,115.6,92.6,67.6,25.1$.

${ }^{19}$ F NMR $\quad\left(470 \mathrm{MHz},\left(\mathrm{CD}_{3}\right)_{2} \mathrm{CO}\right.$, Internal Standard $\left.\mathrm{PhCF}_{3}\right) \delta-142.4$

HRMS (EI-TOF, m/z) calcd. for $\mathrm{C}_{10} \mathrm{H}_{9} \mathrm{BF}_{2} \mathrm{O}_{4}[\mathrm{M}]^{+}$calc.: 241.0598; found: 241.0589.

IR (ATR, neat, $\left.\mathrm{cm}^{-1}\right) 3042(\mathrm{w}), 2922(\mathrm{w}), 1714(\mathrm{w}), 1608(\mathrm{~m}), 1452(\mathrm{~s}), 1335$ (s), $1214(\mathrm{~s})$, 1005 (s), 979 (s), 858 (s), 761 (s).

mp $\quad 203-204{ }^{\circ} \mathrm{C}$.

Synthesis of Methoxy-Tropolone 20: For photochemical approach: To a 1-L quartz vessel was added a

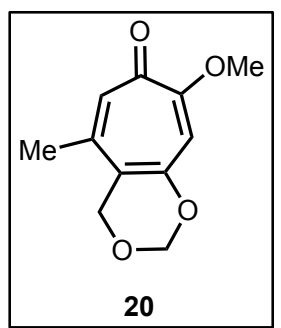
solution of 35 (1.10 g, $4.48 \mathrm{mmol}$.) in benzene $(0.45 \mathrm{~L}, 0.010 \mathrm{M})$. The vessel was sealed with a rubber septum and the solution was degassed with nitrogen for $20 \mathrm{~min}$, then the reaction was irradiated using six UV lamps for $18 \mathrm{~h}$ (for light setup, see $\mathbf{2 2} \rightarrow \mathbf{3 3}$ and Picture S1). Thereafter, the solution became a suspension that was concentrated under reduced pressure to about $100 \mathrm{~mL}$ of benzene, then treated with a saturated aqueous sodium bicarbonate $(25 \mathrm{~mL})$, which led to dissolution of the solid. The aqueous layer was extracted with ethyl acetate $(5 \times 25$ $\mathrm{mL})$ then a 4:1 mixture of ethyl acetate:isopropyl alcohol $(2 \times 25 \mathrm{~mL})$. The combined organic extracts were washed with brine, dried over magnesium sulfate, filtered, and concentrated. The crude product was purified by column chromatography (EtOAc to 9:1 EtOAc: $i$-PrOH) to give $\mathbf{2 0}$ as a tan solid $(0.627 \mathrm{~g}, 2.96$ mmol, 66\%). A mixture of compounds 35 and $\mathbf{3 5}^{\prime}$ (2.20 g, $\left.8.98 \mathrm{mmol}\right)$ in benzene (0.70 L, $\left.0.013 \mathrm{M}\right)$ may 
be converted to compound $\mathbf{2 0}(1.14 \mathrm{~g}, 5.47 \mathrm{mmol}, 61 \%)$ using an analogous procedure. Compound $\mathbf{2 0}$ was recrystallized from dichloromethane to produce crystals suitable for X-ray diffraction analysis.

For silver salt approach: To a solution of $\mathbf{3 5}(0.050 \mathrm{~g}, 0.20 \mathrm{mmol}, 1.00$ equiv.) in dichloromethane $(2.0 \mathrm{~mL})$ cooled to $0{ }^{\circ} \mathrm{C}$ was added $\mathrm{AgBF}_{4}(0.080 \mathrm{~g}, 0.41 \mathrm{mmol}, 2.0$ equiv.). The reaction was allowed to warm to room temperature overnight and thereafter, the heterogeneous reaction was filtered to remove residual salts and directly concentrated and purified by column chromatography (EtOAc to 9:1 EtOAc:i-PrOH) to give 20 as a tan solid (0.033 g, $0.16 \mathrm{mmol}, 78 \%)$. Notably, running this reaction on scales larger than $50 \mathrm{mg}$ led to inconsistent yields.

$\mathbf{R}_{\mathrm{f}}$ $0.4\left(\mathrm{SiO}_{2}, \mathrm{EtOAc}: i-\mathrm{PrOH}=9: 1, \mathrm{UV}, \mathrm{KMnO}_{4}\right)$.

${ }^{1}$ H NMR $\quad\left(500 \mathrm{MHz}, \mathrm{CDCl}_{3}\right) \delta 6.97(\mathrm{~s}, 1 \mathrm{H}), 6.44(\mathrm{~s}, 1 \mathrm{H}), 5.19(\mathrm{~s}, 2 \mathrm{H}), 4.68(\mathrm{~s}, 2 \mathrm{H}), 3.90(\mathrm{~s}, 3 \mathrm{H})$, $2.16(\mathrm{~s}, 3 \mathrm{H})$.

${ }^{13}$ C NMR $\quad\left(126 \mathrm{MHz}, \mathrm{CDCl}_{3}\right) \delta 177.7,162.3,155.8,145.1,132.1,116.4,109.7,90.4,65.8,56.2,23.9$.

HRMS (ESI-TOF, m/z) calcd. for $\mathrm{C}_{11} \mathrm{H}_{13} \mathrm{O}_{4}[\mathrm{M}+\mathrm{H}]^{+}$calc.: 209.0814; found: 209.0807.

IR ATR, neat, $\left.\mathrm{cm}^{-1}\right) 3004(\mathrm{w}), 2907$ (w), 2848 (w), 1608 (s), 1562 (s), 1494 (s), 1431 (m), 1280 (s), 1181 (s), 1016 (s), 974 (s), 963 (s), 893 (m), 673 (m), 607 (m), 549 (s).

mp $\quad 146-150^{\circ} \mathrm{C}$.

Synthesis of Hydroxyketone 40: To a solution of (-)-kobusone (24, 20 g, 90 mmol, 1.0 equiv. $)^{9}$ in

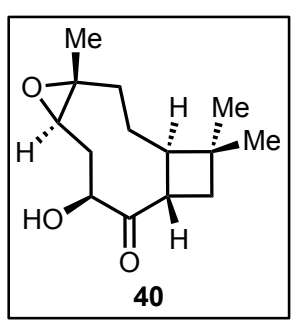
anhydrous THF $(450 \mathrm{~mL})$ cooled to $-78{ }^{\circ} \mathrm{C}$ was added dropwise freshly prepared KHMDS (198 mL, 0.5 M in PhMe, $99 \mathrm{mmol}, 1.10$ equiv.) — the use of a freshly prepared solution is crucial, as commercial solutions led to severely diminished yields. The resulting yellow solution was stirred at $-78{ }^{\circ} \mathrm{C}$ for a half hour and thereafter treated dropwise with a solution of Davis' oxaziridine (28.2 g, $108 \mathrm{mmol}, 1.2$ equiv.) in anhydrous THF (50 $\mathrm{mL})$. The contents were stirred an additional three hours at $-78^{\circ} \mathrm{C}$ and thereafter quenched with saturated aqueous ammonium chloride $(300 \mathrm{~mL})$. After warming to room temperature, the THF was removed in vacuo. The aqueous layer was extracted with ethyl acetate $(3 \times 300 \mathrm{~mL})$, and the combined organics were washed with brine $(800 \mathrm{~mL})$, dried over magnesium sulfate, filtered, and concentrated. The crude oil was purified via flash chromatography (silica gel, hexane:EtOAc $=10: 1$ to 1:1) to afford the $\mathbf{4 0}$ as a white solid 
(18.6 g, $78.0 \mathrm{mmol}, 87 \%$ ). Crystals suitable for X-ray crystallographic analysis were grown from dichloromethane and methanol.

$\mathbf{R}_{\mathbf{f}} \quad 0.5\left(\mathrm{SiO}_{2}\right.$, hexanes:EtOAc $\left.=1: 1, \mathrm{KMnO}_{4}\right)$.

${ }^{1} \mathbf{H}$ NMR $\quad\left(500 \mathrm{MHz}, \mathrm{CDCl}_{3}\right) \delta 4.73-4.68(\mathrm{~m}, 1 \mathrm{H}), 3.43(\mathrm{q}, J=9.5 \mathrm{~Hz}, 1 \mathrm{H}), 2.83(\mathrm{dt}, J=12.1,5.0$ $\mathrm{Hz}, 2 \mathrm{H}), 2.67-2.60(\mathrm{~m}, 1 \mathrm{H}), 2.20(\mathrm{t}, J=10.3 \mathrm{~Hz}, 1 \mathrm{H}), 2.15(\mathrm{dt}, J=13.0,3.8 \mathrm{~Hz}, 1 \mathrm{H})$, $1.96(\mathrm{t}, J=10.4 \mathrm{~Hz}, 1 \mathrm{H}), 1.85(\mathrm{dd}, J=10.9,8.8 \mathrm{~Hz}, 1 \mathrm{H}), 1.73-1.67(\mathrm{~m}, 1 \mathrm{H}), 1.53$ (dddd, $J=15.3,13.3,11.1,4.6 \mathrm{~Hz}, 1 \mathrm{H}), 1.42(\mathrm{ddd}, J=13.2,11.2,7.8 \mathrm{~Hz}, 1 \mathrm{H}), 1.22(\mathrm{~d}, J=0.6$ $\mathrm{Hz}, 3 \mathrm{H}), 1.08(\mathrm{~s}, 6 \mathrm{H}), 1.02(\mathrm{td}, J=13.2,4.8 \mathrm{~Hz}, 1 \mathrm{H})$.

${ }^{13} \mathbf{C ~ N M R ~} \quad\left(126 \mathrm{MHz}, \mathrm{CDCl}_{3}\right) \delta 215.2,71.4,60.1,59.9,47.9,47.5,38.3,37.6,35.6,35.0,29.8,26.5$, 21.8, 16.6 .

HRMS (ESI-TOF, m/z) calcd. For $\mathrm{C}_{14} \mathrm{H}_{22} \mathrm{O}_{3}[\mathrm{M}+\mathrm{Na}]^{+}$calc.: 261.1461; found: 261.1455.

IR

(ATR, neat, $\left.\mathrm{cm}^{-1}\right) 3469$ (w), 2935 (s), 2866 (w), 1697 (s), 1448 (m), 1340 (m), 1164 (s), $1066(\mathrm{~m})$.

mp $\quad 97-99^{\circ} \mathrm{C}$.

$[\boldsymbol{\alpha}]_{\mathbf{D}}{ }^{23}-4.4\left(\mathrm{c}=1.00\right.$ in $\left.\mathrm{CHCl}_{3}\right)$.

Synthesis of Silyl Ether S1: To a solution of hydroxyketone 40 (16.35 g, 68.6 mmol, 1.0 equiv.) and

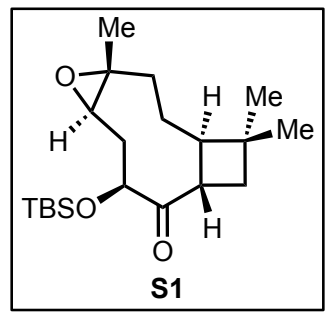
imidazole (20.7 g, $137.2 \mathrm{mmol}, 3.0$ equiv.) in anhydrous DMF (275 mL) was added TBSCl (14.0 g, $205.8 \mathrm{mmol}, 2.0$ equiv.). The resulting yellow solution was heated at $100{ }^{\circ} \mathrm{C}$ for 12 hours. Thereafter, the reaction was cooled in an ice bath and quenched with saturated aqueous sodium bicarbonate $(275 \mathrm{~mL})$ and the aqueous phase was extracted with diethyl ether $(3 \times 300 \mathrm{~mL})$. The combined organics were washed with brine $(900 \mathrm{~mL})$, dried over magnesium sulfate, filtered, and concentrated. The crude oil was purified via flash chromatography ( silica gel, hexanes:EtOAc $=20: 1)$ to afford $\mathbf{S 1}$ as a clear oil $(19.74 \mathrm{~g}, 78.0 \mathrm{mmol}, 81 \%)$.

$\mathbf{R}_{\mathbf{f}} \quad 0.4\left(\mathrm{SiO}_{2}\right.$, hexanes:EtOAc $\left.=10: 1, \mathrm{KMnO}_{4}\right)$.

${ }^{1}$ H NMR $\quad\left(500 \mathrm{MHz}, \mathrm{CDCl}_{3}\right) \delta 4.31(\mathrm{dd}, J=7.2,1.0 \mathrm{~Hz}, 1 \mathrm{H}), 3.92(\mathrm{dt}, J=9.4,8.3 \mathrm{~Hz}, 1 \mathrm{H}), 2.65$ (ddd, $J=13.9,7.2,5.3 \mathrm{~Hz}, 1 \mathrm{H}), 2.47(\mathrm{dd}, J=10.7,5.3 \mathrm{~Hz}, 1 \mathrm{H}), 2.12(\mathrm{dt}, J=13.1,3.7 \mathrm{~Hz}$, $1 \mathrm{H}), 2.03(\mathrm{dd}, J=10.6,9.4 \mathrm{~Hz}, 1 \mathrm{H}), 1.91(\mathrm{ddd}, J=11.3,6.6,3.2 \mathrm{~Hz}, 1 \mathrm{H}), 1.62-1.52(\mathrm{~m}$, $3 \mathrm{H}), 1.41(\mathrm{~s}, 3 \mathrm{H}), 1.36(\mathrm{ddd}, J=13.9,10.7,1.0 \mathrm{~Hz}, 1 \mathrm{H}), 1.05(\mathrm{~s}, 3 \mathrm{H}), 1.04(\mathrm{~s}, 3 \mathrm{H}), 0.94-$ $0.84(\mathrm{~m}, 10 \mathrm{H}), 0.04(\mathrm{~s}, 3 \mathrm{H}), 0.00(\mathrm{~s}, 3 \mathrm{H})$.

${ }^{13}$ C NMR $\quad\left(126 \mathrm{MHz}, \mathrm{CDCl}_{3}\right) \delta 217.2,75.9,60.2,60.1,51.9,41.0,39.7,36.1,34.2,34.1,29.3,26.5$, $25.8,23.0,18.2,18.0,-4.9,-5.1$.

HRMS (ESI-TOF, m/z) calcd. For $\mathrm{C}_{20} \mathrm{H}_{36} \mathrm{O}_{3} \mathrm{Si}[\mathrm{M}+\mathrm{Na}]^{+}$calc.: 375.2326; found: 375.2322 .

IR

ATR, neat, $\left.\mathrm{cm}^{-1}\right) 2952(\mathrm{w}), 2930(\mathrm{w}), 2858(\mathrm{w}), 1703(\mathrm{w}), 1471(\mathrm{w}), 1254(\mathrm{w}), 111(\mathrm{~m})$, 
$1050(\mathrm{~m}), 907(\mathrm{~m}), 829(\mathrm{~m}), 777(\mathrm{~m})$.

$[\boldsymbol{\alpha}]_{\mathbf{D}}{ }^{23}-73.8\left(\mathrm{c}=1.00\right.$ in $\left.\mathrm{CHCl}_{3}\right)$.

Synthesis of Allylic Ether 23: To a flask containing a solution of methyltriphenylphosphonium bromide

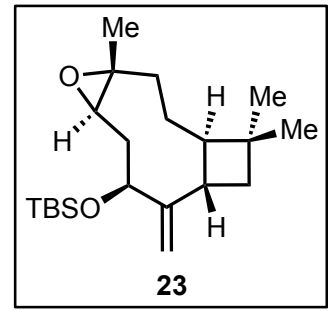

(31.6 g, $84.0 \mathrm{mmol}, 1.5$ equiv., $95 \%)$ in anhydrous $\mathrm{THF}(200 \mathrm{~mL})$ at $0{ }^{\circ} \mathrm{C}$ was added dropwise $n$-butyllithium (49.0 mL, 1.6 M in hexanes, $78.4 \mathrm{mmol}, 1.4$ equiv.). The resulting heterogeneous orange solution was stirred at this temperature for 30 minutes and thereafter treated dropwise with a solution of silyl ether S1 (19.74 g,

$56.0 \mathrm{mmol}, 1.00$ equiv. $)$ in anhydrous THF $(100 \mathrm{~mL})$. The contents were warmed to room temperature and allowed to stir an additional $12 \mathrm{~h}$. Thereafter, the reaction was quenched with saturated aqueous ammonium chloride $(100 \mathrm{~mL})$, THF was removed in vacuo and extracted with ethyl acetate $(3 \times 300 \mathrm{~mL})$. The combined organics were washed with saturated aqueous brine $(900 \mathrm{~mL})$, dried over magnesium sulfate, filtered, and concentrated. The crude material was purified via flash chromatography (silica gel, hexanes:EtOAc $=20: 1$, $\left.\mathrm{KMnO}_{4}\right)$ to afford 23 as a colorless oil $(18.47 \mathrm{~g}, 52.7 \mathrm{mmol}, 94 \%)$.

$\mathbf{R}_{\mathbf{f}} \quad 0.5\left(\mathrm{SiO}_{2}\right.$, hexanes:EtOAc $\left.=10: 1, \mathrm{KMnO}_{4}\right)$.

${ }^{1}$ H NMR $\quad\left(500 \mathrm{MHz} \mathrm{CDCl}_{3}\right) \delta 5.07(\mathrm{~s}, 1 \mathrm{H}), 5.05(\mathrm{~s}, 1 \mathrm{H}), 4.57(\mathrm{dd}, J=6.2,3.4 \mathrm{~Hz}, 1 \mathrm{H}), 3.09-2.98$ (m, 1H), $2.75(\mathrm{dd}, J=10.8,4.5 \mathrm{~Hz}, 1 \mathrm{H}), 2.44$ (dddd, $J=13.4,6.2,4.6,0.8 \mathrm{~Hz}, 1 \mathrm{H}), 2.09$ (dt, $J=12.9,3.6 \mathrm{~Hz}, 1 \mathrm{H}), 1.76(\mathrm{ddd}, J=10.9,8.8,0.8 \mathrm{~Hz}, 1 \mathrm{H}), 1.71-1.64(\mathrm{~m}, 2 \mathrm{H}), 1.58$ $(\mathrm{dtd}, J=14.7,4.1,1.9 \mathrm{~Hz}, 1 \mathrm{H}), 1.53-1.40(\mathrm{~m}, 2 \mathrm{H}), 1.30(\mathrm{~s}, 3 \mathrm{H}), 1.03(\mathrm{~s}, 3 \mathrm{H}), 1.01$ (s, $3 \mathrm{H}), 0.97-0.90(\mathrm{~m}, 1 \mathrm{H}), 0.89(\mathrm{~s}, 9 \mathrm{H}), 0.03(\mathrm{~s}, 3 \mathrm{H}),-0.02(\mathrm{~s}, 3 \mathrm{H})$.

${ }^{13} \mathbf{C ~ N M R ~} \quad\left(126 \mathrm{MHz}, \mathrm{CDCl}_{3}\right) \delta 155.9,110.9,71.9,61.3,60.5,53.8,41.3,39.9,38.6,38.4,33.3,30.2$, $27.5,25.9,22.7,18.3,17.8,-4.8,-5.0$.

HRMS (ESI-TOF, m/z) calcd. For $\mathrm{C}_{21} \mathrm{H}_{38} \mathrm{O}_{2} \mathrm{Si}[\mathrm{M}+\mathrm{Na}]^{+}$calc.: 373.2533; found: 373.2528 .

IR (ATR, neat, $\left.\mathrm{cm}^{-1}\right) 2952(\mathrm{w}), 2928(\mathrm{w}), 2858(\mathrm{w}), 1472(\mathrm{w}), 1387(\mathrm{w}), 1255(\mathrm{w}), 1046(\mathrm{w})$, $830(\mathrm{~m}), 775(\mathrm{~m})$.

$[\alpha]_{\mathbf{D}}{ }^{23}-47.5\left(\mathrm{c}=1.00\right.$ in $\left.\mathrm{CHCl}_{3}\right)$.

Synthesis of Epoxide 41: Procedure done in accordance to the previous literature report. ${ }^{10}$ To a flask

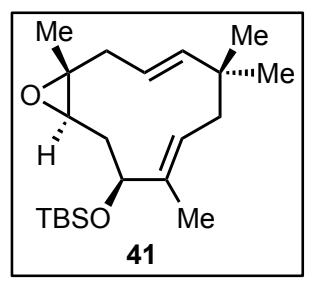
charged with $23(18.47 \mathrm{~g}, 52.68 \mathrm{mmol})$ and $\mathrm{Co}\left(\mathrm{sal}^{t-\mathrm{Bu}, t-\mathrm{Bu}}\right) \mathrm{Cl}(0.673 \mathrm{~g}, 1.05 \mathrm{mmol}$, 0.02 equiv.) was added benzene $(263 \mathrm{~mL})$ and was thereafter degassed with argon for one hour at $0{ }^{\circ} \mathrm{C}$. After warming to room temperature, degassed $\mathrm{PhSiH}_{3}(0.26 \mathrm{~mL}$, 2.10 mmol, 0.04 equiv.) was added to the reaction, which immediately turned from 
dark green to bright red. The contents were allowed to stir at room temperature 24 hours. Thereafter, solvent was removed in vacuo and the resulting crude oil was purified via flash chromatography (silica gel, hexanes:EtOAc $=20: 1)$ to afford 41 as a dark red solid $(17.56 \mathrm{~g}, 50.1 \mathrm{mmol}, 95 \%)$.

$\mathbf{R}_{\mathbf{f}} \quad 0.6\left(\mathrm{SiO}_{2}\right.$, hexanes:EtOAc $\left.=10: 1, \mathrm{KMnO}_{4}\right)$.

${ }^{1}$ H NMR $\quad\left(500 \mathrm{MHz}, \mathrm{CDCl}_{3}\right) \delta 5.27(\mathrm{~m}, 2 \mathrm{H}), 5.19(\mathrm{~d}, J=16.1 \mathrm{~Hz}, 1 \mathrm{H}), 4.22(\mathrm{dd}, J=7.7,5.0 \mathrm{~Hz}$, $1 \mathrm{H}), 2.56-2.46(\mathrm{~m}, 2 \mathrm{H}), 2.20(\mathrm{~d}, J=13.8 \mathrm{~Hz}, 1 \mathrm{H}), 2.04(\mathrm{t}, J=7.1 \mathrm{~Hz}, 1 \mathrm{H}), 1.93(\mathrm{dd}, J=$ 13.8, 6.2 Hz, 1H), 1.70 (dd, $J=12.8,9.1 \mathrm{~Hz}, 1 \mathrm{H}), 1.58-1.47(\mathrm{~m}, 4 \mathrm{H}), 1.29$ (s, 3H), 1.11 $(\mathrm{s}, 3 \mathrm{H}), 1.09(\mathrm{~s}, 3 \mathrm{H}), 0.87(\mathrm{~s}, 9 \mathrm{H}), 0.03(\mathrm{~s}, 3 \mathrm{H}), 0.00(\mathrm{~s}, 3 \mathrm{H})$.

${ }^{13} \mathrm{C}$ NMR $\quad\left(126 \mathrm{MHz}, \mathrm{DMSO}-d 6,80{ }^{\circ} \mathrm{C}\right) \delta 141.6,134.6,122.9,122.4,75.3,62.4,58.5,40.6,40.1$, $35.3,33.3,27.6,26.7,25.3,17.9,17.4,10.9,-5.44,-5.39$.

HRMS (ESI-TOF, m/z) calcd. For $\mathrm{C}_{21} \mathrm{H}_{38} \mathrm{O}_{2} \mathrm{Si}\left[\mathrm{M}+\mathrm{NH}_{4}\right]^{+}$calc.: 368.2979; found: 368.2965 .

IR $\quad\left(A T R\right.$, neat, $\left.\mathrm{cm}^{-1}\right) 2954(\mathrm{w}), 2927(\mathrm{w}), 2856(\mathrm{w}), 1470(\mathrm{w}), 1249(\mathrm{w}), 1069(\mathrm{~m}), 1047(\mathrm{~m})$, $900(\mathrm{~m}), 833(\mathrm{~m}), 779(\mathrm{~m})$.

mp $\quad 40-42{ }^{\circ} \mathrm{C}$.

$[\alpha]_{\mathbf{D}}{ }^{23} \quad-77.2\left(\mathrm{c}=1.00\right.$ in $\left.\mathrm{CHCl}_{3}\right)$.

Synthesis of Epoxy-Alcohol 42: To a solution of epoxide 41 (6.7 g, 19.2 mmol, 1.0 equiv.) in anhydrous

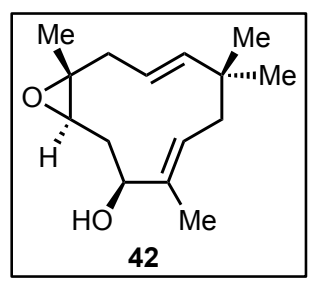
THF $(38 \mathrm{~mL})$ cooled to $0{ }^{\circ} \mathrm{C}$ was added dropwise TBAF $(28.8 \mathrm{~mL}, 1.0 \mathrm{M}$ in THF, $28.8 \mathrm{mmol}, 1.5$ equiv.). The contents were warmed to room temperature and stirred 24 hours. Thereafter, the reaction was quenched with saturated aqueous ammonium chloride $(20 \mathrm{~mL})$ and THF was removed in vacuo. The organics were extracted with ethyl acetate $(3 \times 100 \mathrm{~mL})$ and the combined organics were washed with brine $(250$ $\mathrm{mL}$ ), dried over magnesium sulfate, filtered, and concentrated. The crude oil was purified via flash chromatography $\left(\mathrm{SiO}_{2}\right.$, hexanes:EtOAc $\left.=4: 1-2: 1, \mathrm{KMnO}_{4}\right)$ to provide the title compound as a white solid (4.5 g, $19.0 \mathrm{mmol}, 99 \%)$.

$\mathbf{R}_{\mathbf{f}} \quad 0.1\left(\mathrm{SiO}_{2}\right.$, hexanes:EtOAc $\left.=4: 1, \mathrm{KMnO}_{4}\right)$.

${ }^{1}$ H NMR $\quad\left(500 \mathrm{MHz}, \mathrm{CDCl}_{3}\right) \delta 5.38-5.31(\mathrm{~m}, 1 \mathrm{H}), 5.29(\mathrm{dd}, J=8.8,5.5 \mathrm{~Hz}, 1 \mathrm{H}), 5.24(\mathrm{~d}, J=16.1$ $\mathrm{Hz}, 1 \mathrm{H}), 4.34(\mathrm{ddd}, J=8.3,4.4,2.9 \mathrm{~Hz}, 1 \mathrm{H}), 2.57-2.51(\mathrm{~m}, 2 \mathrm{H}), 2.34-2.26(\mathrm{~m}, 1 \mathrm{H})$, $2.12(\mathrm{dd}, J=13.7,9.5 \mathrm{~Hz}, 1 \mathrm{H}), 1.96(\mathrm{dd}, J=13.9,5.5 \mathrm{~Hz}, 1 \mathrm{H}), 1.75(\mathrm{dd}, J=12.9,8.8 \mathrm{~Hz}$, $1 \mathrm{H}), 1.64-1.58(\mathrm{~m}, 1 \mathrm{H}), 1.57(\mathrm{~d}, J=1.4 \mathrm{~Hz}, 3 \mathrm{H}), 1.52(\mathrm{~d}, J=3.1 \mathrm{~Hz}, 1 \mathrm{H}), 1.32(\mathrm{~s}, 3 \mathrm{H})$, 1.15 (s, 3H), 1.13 (s, 3H).

${ }^{13}$ C NMR $\quad\left(126 \mathrm{MHz}, \mathrm{CDCl}_{3}\right) \delta 142.6,135.1,124.9,123.1,76.0,63.9,60.3,41.2,40.9,36.3,32.4$, 28.6, 27.2, 18.7, 11.6. 
Note: While all the carbon signals are present in the spectrum, the broadening of peaks, due to the conformations of the macrocycle, was not able to be resolved with temperature variations during the experiment as seen in other macrocycles.

HRMS (ESI-TOF, m/z) calcd. For $\mathrm{C}_{15} \mathrm{H}_{24} \mathrm{O}_{2}[\mathrm{M}+\mathrm{Na}]^{+}$calc.: 259.1669; found: 259.1667.

IR $\quad\left(\right.$ ATR, neat, $\left.\mathrm{cm}^{-1}\right) 3427$ (br), $2958(\mathrm{~s}), 1449(\mathrm{~m}), 1385(\mathrm{~m}), 1021(\mathrm{~m}), 972(\mathrm{~m}), 899(\mathrm{w})$.

mp $\quad 83-85^{\circ} \mathrm{C}$.

$[\alpha]_{\mathbf{D}}{ }^{23} \quad-150.6\left(\mathrm{c}=1.00\right.$ in $\left.\mathrm{CHCl}_{3}\right)$.

Synthesis of Triene S2: Procedure done in accordance to the previous literature report. ${ }^{11}$ To a pressure

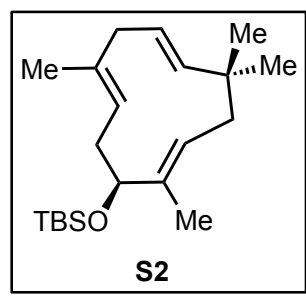
tube charged with epoxide 41 (3.54 g, 1.10 mmol, 1.0 equiv.), and rhenium(VII) oxide (0.122 g, $0.252 \mathrm{mmol}, 0.025$ equiv.) was added anhydrous PhMe (33 mL) followed by $\mathrm{P}(\mathrm{OPh})_{3}(2.8 \mathrm{~mL}, 10.6 \mathrm{mmol}, 1.05$ equiv.). The resulting mixture was heated at $100{ }^{\circ} \mathrm{C}$ for three hours and thereafter cooled to room temperature. The solvent was removed in vacuo and the crude material was filtered through a short pad of silica rinsing with hexanes and thereafter purified via flash chromatography (silica gel, hexanes:EtOAc $=40: 1-20: 1$ ) to afford $\mathbf{S 2}$ as a pale yellow oil (2.93 g, $8.76 \mathrm{mmol}, 87 \%)$.

$\mathbf{R}_{\mathbf{f}} \quad 0.8\left(\mathrm{SiO}_{2}\right.$, hexanes:EtOAc $\left.=25: 1, \mathrm{KMnO}_{4}\right)$.

${ }^{1}$ H NMR $\quad\left(500 \mathrm{MHz}, \mathrm{CDCl}_{3}\right) \delta 5.52(\mathrm{dt}, J=15.8,7.4 \mathrm{~Hz}, 1 \mathrm{H}), 5.14(\mathrm{~d}, J=15.8 \mathrm{~Hz}, 1 \mathrm{H}), 4.91(\mathrm{~d}, J$ $=10.9 \mathrm{~Hz}, 1 \mathrm{H}), 4.82(\mathrm{t}, J=8.0 \mathrm{~Hz}, 1 \mathrm{H}), 3.95(\mathrm{dd}, J=9.1,6.2 \mathrm{~Hz}, 1 \mathrm{H}), 2.46(\mathrm{~d}, J=7.4 \mathrm{~Hz}$, 2H), 2.27 (ddd, $J=13.4,7.0 \mathrm{~Hz}, 1 \mathrm{H}), 2.12(\mathrm{t}, J=12.4 \mathrm{~Hz}, 1 \mathrm{H}), 2.05$ (ddd, $J=13.2,8.9$ $\mathrm{Hz}, 1 \mathrm{H}), 1.80(\mathrm{~d}, J=12.1 \mathrm{~Hz}, 1 \mathrm{H}), 1.64-1.59(\mathrm{~s}, 3 \mathrm{H}), 1.39$ (t, $J=1.3 \mathrm{~Hz}, 3 \mathrm{H}), 1.05(\mathrm{~s}$, $3 \mathrm{H}), 1.03(\mathrm{~s}, 3 \mathrm{H}), 0.84(\mathrm{~s}, 9 \mathrm{H}), 0.00(\mathrm{~s}, 3 \mathrm{H}),-0.04(\mathrm{~s}, 3 \mathrm{H})$.

${ }^{13} \mathbf{C}$ NMR $\quad\left(126 \mathrm{MHz}\right.$, DMSO- $\left.d 6,90{ }^{\circ} \mathrm{C}\right) \delta 140.5,138.5,134.9,126.5,123.1,122.2,77.9,41.3,39.5$, $36.1,32.5,28.6,25.2,24.2,17.3,17.2,10.1,-5.3$.

HRMS (ESI-TOF, m/z) calcd. For $\mathrm{C}_{21} \mathrm{H}_{38} \mathrm{OSi}[\mathrm{M}+\mathrm{Na}]^{+}$calc.: 357.2584; found: 357.2579 .

IR (ATR, neat, $\left.\mathrm{cm}^{-1}\right) 2955(\mathrm{w}), 2928(\mathrm{w}), 2856(\mathrm{w}), 1472(\mathrm{w}), 1251(\mathrm{w}), 1070(\mathrm{~m}), 1053(\mathrm{~m})$, $967(\mathrm{w}), 834(\mathrm{~m}), 773(\mathrm{~m})$.

$[\alpha]_{\mathbf{D}}{ }^{23} \quad-59.2\left(\mathrm{c}=1.00\right.$ in $\left.\mathrm{CHCl}_{3}\right)$. 
Synthesis of (-)-10-Hydroxyhumulene 11 from Triene S2: To a solution of S2 (1.70 g, $4.85 \mathrm{mmol}, 1.0$

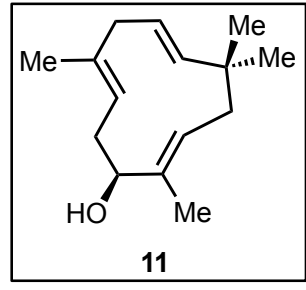
equiv.) in anhydrous THF $(5.1 \mathrm{~mL})$ cooled to $0{ }^{\circ} \mathrm{C}$ was added dropwise TBAF $(9.7$ $\mathrm{mL}, 1.0 \mathrm{M}$ in THF, $9.7 \mathrm{mmol}, 2.0$ equiv.). The contents were warmed to room temperature and stirred 48 hours. Thereafter, the reaction was quenched with saturated aqueous ammonium chloride $(20 \mathrm{~mL})$ and THF was removed in vacuo. The organics were extracted with ethyl acetate $(3 \times 20 \mathrm{~mL})$ and the combined organics were washed with brine $(60 \mathrm{~mL})$, dried over magnesium sulfate, filtered, and concentrated. The crude oil was purified via flash chromatography (silica gel, hexanes:EtOAc $=15: 1$ to $6: 1)$ to afford $\mathbf{1 1}$ as a white solid $(1.05 \mathrm{~g}, 4.44 \mathrm{mmol}$, $92 \%)$.

Synthesis of 10-Hydroxyhumulene 11 from Epoxide 41: To a vial charged with epoxide 41 (0.050 g, $0.14 \mathrm{mmol}, 1.0$ equiv.), and rhenium(VII) oxide ( $0.0021 \mathrm{~g}, 0.0043 \mathrm{mmol}, 0.03$ equiv.) was added anhydrous $\mathrm{PhMe}(0.71 \mathrm{~mL})$ followed by triphenyl phosphite $(0.039 \mathrm{~mL}, 0.14 \mathrm{mmol}, 1.00$ equiv., $97 \%)$. The resulting mixture was heated at $100{ }^{\circ} \mathrm{C}$ for three hours and thereafter cooled to room temperature. The solvent was removed in vacuo and the crude material was treated with TBAF $(0.7 \mathrm{~mL}, 1.0 \mathrm{M}$ in THF, $0.7 \mathrm{mmol}, 5.0$ equiv.). The contents were heated at $50{ }^{\circ} \mathrm{C}$ for 36 hours and thereafter cooled to room temperature and quenched with saturated aqueous ammonium chloride $(2 \mathrm{~mL})$. The organics were extracted with ethyl acetate $(3 \times 4 \mathrm{~mL})$ and the combined organics were washed with brine $(10 \mathrm{~mL})$, dried over magnesium sulfate, filtered, and concentrated. The crude oil was purified via flash chromatography (silica gel, hexanes:EtOAc $=20: 1$ to $6: 1)$ to afford 11 as a white solid $(0.023 \mathrm{~g}, 0.10 \mathrm{mmol}, 73 \%)$.

$\mathbf{R}_{\mathbf{f}} \quad 0.4\left(\mathrm{SiO}_{2}\right.$, hexanes:EtOAc $\left.=4: 1, \mathrm{KMnO}_{4}\right)$.

${ }^{1}$ H NMR $\quad\left(400 \mathrm{MHz}, \mathrm{CDCl}_{3}\right) \delta 5.55(\mathrm{~m}, 1 \mathrm{H}), 5.17(\mathrm{~d}, J=15.8,1 \mathrm{H}), 5.10-5.02(\mathrm{~m}, 1 \mathrm{H}), 4.87(\mathrm{~m}$, $1 \mathrm{H}), 4.07(\mathrm{dd}, J=9.2,6.2 \mathrm{~Hz}, 1 \mathrm{H}), 2.55-2.55(\mathrm{~m}, 3 \mathrm{H}), 2.24-2.03(\mathrm{~m}, 2 \mathrm{H}), 1.85(\mathrm{dd}, J=$ 13.8, 3.4 Hz, 1H), $1.66(\mathrm{~s}, J=1.5 \mathrm{~Hz}, 3 \mathrm{H}), 1.48(\mathrm{t}, J=1.3 \mathrm{~Hz}, 3 \mathrm{H}), 1.08(\mathrm{~s}, 3 \mathrm{H}), 1.07$ (s, $3 \mathrm{H})$.

${ }^{13} \mathrm{C}$ NMR $\quad\left(126 \mathrm{MHz}, \mathrm{DMSO}-d 6,80{ }^{\circ} \mathrm{C}\right) \delta 140.5,137.9,135.8,126.6,122.9,122.6,76.4,41.4,39.5$, $36.2,31.9,28.8,24.2,17.3,10.3$.

HRMS (ESI-TOF, m/z) calcd. For $\mathrm{C}_{15} \mathrm{H}_{24} \mathrm{O}[\mathrm{M}+\mathrm{Na}]^{+}$calc.: 243.1725; found: 243.1724.

IR $\quad\left(A T R\right.$, neat, $\left.\mathrm{cm}^{-1}\right) 3335(\mathrm{br}), 2956(\mathrm{~s}), 2952$ (s), $2862(\mathrm{~s}), 1659(\mathrm{~m}), 1446(\mathrm{~m}), 1383(\mathrm{~m})$, $1362(\mathrm{~m}), 1017(\mathrm{~m}), 966(\mathrm{~m}), 823(\mathrm{~m})$.

mp $\quad 55-56^{\circ} \mathrm{C}$. 
$[\alpha]_{\mathbf{D}}{ }^{23}-121.5\left(\mathrm{c}=1.00\right.$ in $\left.\mathrm{CHCl}_{3}\right) ;-21.1(\mathrm{c}=0.04$ in $\mathrm{MeOH})$.

Note: This value is opposite but nearly equal of that reported by $\mathrm{Hu}^{12}-[\alpha]_{D}^{20}=+17.5, c 0.04, \mathrm{MeOH}$. Thus, we claim that they actually have the enantiomer of the compound they have reported.

Synthesis of Monocycloadduct 43: To a 10-mL microwave vial containing a stir bar was added tropolone

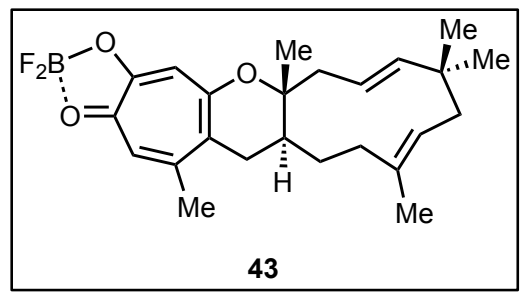
19 (0.100 g, 0.413 mmol, 1.0 equiv.), $\alpha$-humulene (12, $0.253 \mathrm{~g}, 1.24$ mmol, 3.0 equiv.), a crystal of BHT, and mesitylene (4.1 mL, $0.1 \mathrm{M})$. The vial was sealed and heated at $200{ }^{\circ} \mathrm{C}$ for eight hours in a microwave reactor. Tropolone 19 was then determined to be mostly consumed by

TLC (silica gel, 1:1 hexanes:EtOAc). The brown solution was transferred to a recovery flask and concentrated under reduced pressure. The residue was then loaded onto silica gel and purified by flash column chromatography (silica gel, 3:1 hexanes:EtOAc to EtOAc) to give recovered $19(0.0229 \mathrm{~g}, 0.0946$ mmol) and compound 43 (0.0878 g, $0.211 \mathrm{mmol}, 51 \%, 66 \% \mathrm{brsm})$.

$\mathbf{R}_{\mathbf{f}}$ $0.3\left(\mathrm{SiO}_{2}\right.$, hexanes:EtOAc $\left.=2: 1, \mathrm{UV}, \mathrm{KMnO}_{4}\right)$.

${ }^{1}$ H NMR $\quad\left(500 \mathrm{MHz}, \mathrm{CDCl}_{3}\right) \delta 7.33(\mathrm{~s}, 1 \mathrm{H}), 7.25(\mathrm{~s}, 1 \mathrm{H}), 5.18(\mathrm{dd}, J=15.8,2.1 \mathrm{~Hz}, 1 \mathrm{H}), 5.02(\mathrm{dd}, J$ $=12.2,4.1 \mathrm{~Hz}, 1 \mathrm{H}), 4.98(\mathrm{ddd}, J=15.8,10.6,2.1 \mathrm{~Hz}, 1 \mathrm{H}), 2.95(\mathrm{dd}, J=17.9,5.1 \mathrm{~Hz}, 1 \mathrm{H})$, $2.66(\mathrm{dt}, J=14.8,2.1 \mathrm{~Hz}, 1 \mathrm{H}), 2.60(\mathrm{~s}, 3 \mathrm{H}), 2.40(\mathrm{dd}, J=17.9,12.8 \mathrm{~Hz}, 1 \mathrm{H}), 2.34(\mathrm{dd}, J=$ $14.8,10.6 \mathrm{~Hz}, 1 \mathrm{H}), 2.21-2.14(\mathrm{~m}, 2 \mathrm{H}), 1.90(\mathrm{ddd}, J=12.8,8.0,5.1 \mathrm{~Hz}, 1 \mathrm{H}), 1.82(\mathrm{dd}, J$ $=12.0,11.5 \mathrm{~Hz}, 1 \mathrm{H}), 1.77(\mathrm{dd}, J=12.2,4.1 \mathrm{~Hz}, 1 \mathrm{H}), 1.63(\mathrm{~s}, 3 \mathrm{H}), 1.37(\mathrm{dd}, J=13.9,11.5$ $\mathrm{Hz}, 1 \mathrm{H}), 1.23$ (dd, $J=15.0,8.0 \mathrm{~Hz}, 1 \mathrm{H}), 1.19(\mathrm{~s}, 3 \mathrm{H}), 1.06$ (s, 3H), 1.00 (s, 3H).

${ }^{13} \mathbf{C ~ N M R ~} \quad\left(126 \mathrm{MHz}, \mathrm{CDCl}_{3}\right) \delta 168.8,167.6,167.6,156.4,144.2,136.1,128.8,123.8,120.7,119.0$, $115.7,85.1,42.7,41.5,38.4,37.8,34.5,31.6,30.1,29.8,28.1,24.3,20.6,17.2$.

${ }^{19}$ F NMR $\quad\left(470 \mathrm{MHz}, \mathrm{CDCl}_{3}\right.$, Internal Standard $\left.\mathrm{PhCF}_{3}\right) \delta-143.0$

HRMS (EI-TOF, $\mathrm{m} / \mathrm{z}$ ) calcd. for $\mathrm{C}_{24} \mathrm{H}_{31} \mathrm{BF}_{2} \mathrm{O}_{3}[\mathrm{M}-\mathrm{H}]^{+}$calc.: 415.23706; found: 415.23662.

IR (ATR, neat, $\left.\mathrm{cm}^{-1}\right) 2954(\mathrm{w}), 2932(\mathrm{w}), 1618(\mathrm{~m}), 1524(\mathrm{w}), 1371(\mathrm{~m}), 1360(\mathrm{~m}), 1195(\mathrm{~m})$, 1046 (s), $986(\mathrm{~m}), 903(\mathrm{~m}), 836(\mathrm{~m}), 734(\mathrm{~m}), 562(\mathrm{w}), 518(\mathrm{w}), 469(\mathrm{w})$.

mp $\quad 252-255^{\circ} \mathrm{C}$.

Monocycloadduct 44: To a 35-mL microwave vial containing a stir bar was added tropolone $20(0.150 \mathrm{~g}$,

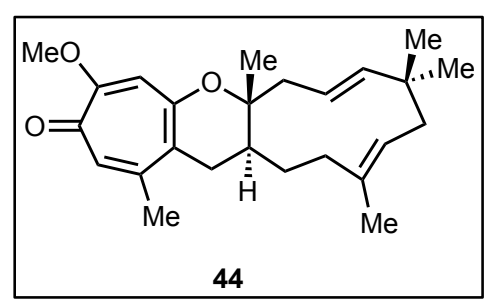
0.720 mmol, 1.0 eq.), $\alpha$-humulene (12, $0.442 \mathrm{~g}, 3.60 \mathrm{mmol}, 3.0$ eq.), a crystal of BHT, and mesitylene (7.2 mL, 0.1 M). The vial was sealed and heated at $200{ }^{\circ} \mathrm{C}$ for 16 hours in a microwave reactor. Tropolone 20 was then determined to be consumed by TLC (silica gel, 9:1 EtOAc:i-PrOH). The brown solution was transferred to a recovery flask and concentrated 
under reduced pressure. The residue was then loaded onto silica gel and purified by flash column chromatography (silica gel, 1:3 hexanes:EtOAc to pure EtOAc) to give compound $\mathbf{4 4}(0.225 \mathrm{~g}, 0.588 \mathrm{mmol}$, $82 \%)$.

$\mathbf{R}_{\mathbf{f}} \quad 0.6\left(\mathrm{SiO}_{2}, \mathrm{EtOAc}, \mathrm{UV}, \mathrm{KMnO}_{4}\right)$.

${ }^{1}$ H NMR $\quad\left(500 \mathrm{MHz}, \mathrm{CDCl}_{3}\right) \delta 7.01(\mathrm{~s}, 1 \mathrm{H}), 6.50(\mathrm{~s}, 1 \mathrm{H}), 5.14(\mathrm{dd}, J=15.8,1.6 \mathrm{~Hz}, 1 \mathrm{H}), 5.08(\mathrm{ddd}$, $J=16.0,9.9,2.3 \mathrm{~Hz}, 1 \mathrm{H}), 5.02(\mathrm{dd}, J=11.5,3 \mathrm{~Hz}, 1 \mathrm{H}), 3.89$ (s, 3H), 2.77 (dd, $J=17.5$, $5.4 \mathrm{~Hz}, 1 \mathrm{H}), 2.53(\mathrm{~d}, J=14.5 \mathrm{~Hz}, 1 \mathrm{H}), 2.30(\mathrm{~s}, 3 \mathrm{H}), 2.30-2.24(\mathrm{~m}, 1 \mathrm{H}), 2.20-2.09(\mathrm{~m}$, $3 \mathrm{H}), 1.83-1.73(\mathrm{~m}, 3 \mathrm{H}), 1.61(\mathrm{~s}, 3 \mathrm{H}), 1.32(\mathrm{dd}, J=13.8,10.9 \mathrm{~Hz}, 1 \mathrm{H}), 1.13(\mathrm{dt}, J=14.2$, $8.3 \mathrm{~Hz}, 1 \mathrm{H}), 1.08(\mathrm{~s}, 3 \mathrm{H}), 1.05(\mathrm{~s}, 3 \mathrm{H}), 1.03(\mathrm{~s}, 3 \mathrm{H})$.

${ }^{13}$ C NMR $\quad\left(126 \mathrm{MHz}, \mathrm{CDCl}_{3}\right) \delta 177.6,161.6,156.8,148.5,143.1,136.4,130.5,123.4,120.1,117.5$, $111.3,81.3,56.0,42.7,41.5,38.4,38.0,35.1,30.6,30.3,30.3,26.8,24.4,20.1,17.2$.

HRMS (EI-TOF, m/z) calcd. for $\mathrm{C}_{25} \mathrm{H}_{35} \mathrm{O}_{3}[\mathrm{M}+\mathrm{H}]^{+}$calc.: 383.2586; found: 383.2579.

IR (ATR, neat, $\left.\mathrm{cm}^{-1}\right) 2929(\mathrm{w}), 2857(\mathrm{w}), 1737(\mathrm{w}), 1602(\mathrm{~s}), 1566(\mathrm{~s}), 1486(\mathrm{~s}), 1454(\mathrm{~m})$, 1426 (m), 1266 (m), 1177 (s), 1151 (s), 1011 (s), 875 (m), 729 (w).

mp

$147-150{ }^{\circ} \mathrm{C}$.

Synthesis of Dehydroxyepolone B 15' from 43: To a solution of monocycloadduct $43(0.0318 \mathrm{~g}, 73.6$

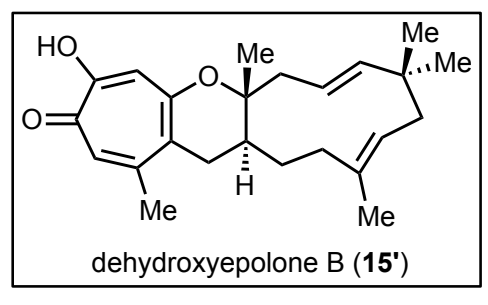

mmol, 1.0 equiv. $)$ in methanol $(0.74 \mathrm{~mL}, 0.1 \mathrm{M})$ was added potassium carbonate (0.102 g, $0.736 \mathrm{mmol}, 10.0$ equiv.). The suspension was vigorously stirred until the starting material was consumed. After 24 hours, the reaction was transferred to a separatory funnel containing $\mathrm{pH}=$ 7 phosphate buffer $(2 \mathrm{~mL})$ and washed with ethyl acetate $(2 \mathrm{~mL})$. The aqueous layer was drained and acidified with citric acid until a neutral $\mathrm{pH}$ was obtained. The aqueous layer was transferred back into the separatory funnel and extracted with the previously retained organic extract. The aqueous layer was subsequently washed with ethyl acetate $(3 \times 2 \mathrm{~mL})$, then the combined organic extracts were washed with brine (4 mL), dried over magnesium sulfate, filtered, and concentrated under reduced pressure to give $\mathbf{1 5}^{\prime}$ as an orange solid (0.0283 $\mathrm{g}, 0.0736 \mathrm{mmol}, 99 \%)$.

Synthesis of 15' from 44: To a 4-mL vial containing a magnetic stir bar and 44 (0.0250 g, $0.0654 \mathrm{mmol}$, 1.0 equiv. $)$ was added methanol $(0.65 \mathrm{~mL})$ then a solution of sodium hydroxide $(1.0 \mathrm{M}, 0.65 \mathrm{~mL}, 0.65$ mmol, 10 equiv.). The reaction was heated at $100{ }^{\circ} \mathrm{C}$ for eight hours, after which the reaction was checked by TLC (EtOAc) and the starting material spot became an undefined streak. The reaction was quenched 
with $\mathrm{pH}=7$ phosphate buffer $(1.0 \mathrm{~mL})$, then $1 \mathrm{M} \mathrm{HCl}(0.65 \mathrm{~mL})$ was added to neutralize the $\mathrm{pH}$ of the crude reaction. The aqueous mixture was transferred to a separatory funnel and extracted with ethyl acetate $(3 \times$ $1 \mathrm{~mL})$, then the combined organic extracts were washed with brine $(2 \mathrm{~mL})$, dried over magnesium sulfate, filtered, and concentrated, to give $\mathbf{1 5}^{\prime}$ as an orange solid $(0.0193 \mathrm{~g}, 0.052 \mathrm{mmol}, 80 \%)$.

$\mathbf{R}_{\mathbf{f}}$

${ }^{1}$ H NMR

${ }^{13}$ C NMR

HRMS

IR

$\mathbf{m p}$

$0.4\left(\mathrm{SiO}_{2}, \mathrm{CH}_{2} \mathrm{Cl}_{2}: \mathrm{MeOH}=19: 1, \mathrm{UV}, \mathrm{KMnO}_{4}\right)$.

$\left(500 \mathrm{MHz}, \mathrm{CDCl}_{3}\right) \delta 7.13(\mathrm{~s}, 1 \mathrm{H}), 7.01(\mathrm{~s}, 1 \mathrm{H}), 5.14(\mathrm{dd}, J=15.9,1.6 \mathrm{~Hz}, 1 \mathrm{H}), 5.07$ (ddd, $J=16.0,10.1,2.6 \mathrm{~Hz}, 1 \mathrm{H}), 5.02(\mathrm{dd}, J=11.5,3.0 \mathrm{~Hz}, 1 \mathrm{H}), 2.82(\mathrm{dd}, J=17.4,5.2 \mathrm{~Hz}, 1 \mathrm{H})$, $2.56(\mathrm{~d}, J=14.3 \mathrm{~Hz}, 1 \mathrm{H}), 2.40(\mathrm{~s}, 3 \mathrm{H}), 2.28(\mathrm{dd}, J=14.5,10.1 \mathrm{~Hz}, 1 \mathrm{H}), 2.22(\mathrm{dd}, J=17.4$, $12.2 \mathrm{~Hz}, 1 \mathrm{H}), 2.20-2.11(\mathrm{~m}, 2 \mathrm{H}), 1.86-1.74(\mathrm{~m}, 3 \mathrm{H}), 1.62(\mathrm{~s}, 3 \mathrm{H}), 1.34(\mathrm{dd}, J=13.8$, $11.0 \mathrm{~Hz}, 1 \mathrm{H}), 1.20-1.12(\mathrm{~m}, 1 \mathrm{H}), 1.11(\mathrm{~s}, 3 \mathrm{H}), 1.05(\mathrm{~s}, 3 \mathrm{H}), 1.02(\mathrm{~s}, 3 \mathrm{H})$.

$\left(126 \mathrm{MHz}, \mathrm{CDCl}_{3}\right) \delta 172.7,163.3,161.5,150.2,143.3,136.4,124.6,123.7,120.9,120.2$, $113.6,82.0,43.0,41.7,38.5,38.1,35.2,31.2,30.3,29.9,27.5,24.6,20.4,17.4$.

(EI-TOF, m/z) calcd. for $\mathrm{C}_{24} \mathrm{H}_{32} \mathrm{O}_{3}[\mathrm{M}]^{+}$calc.: 368.23514; found: 368.23532 .

(ATR, neat, $\left.\mathrm{cm}^{-1}\right) 3180(\mathrm{w}), 2925(\mathrm{~m}), 2856(\mathrm{~m}), 1733(\mathrm{w}), 1588(\mathrm{~m}), 1438(\mathrm{~s}), 1367(\mathrm{~m})$, 1280 (m), 1756 (s), 1134 (s), 1046 (s), 890 (m), 747 (w).

$181-184{ }^{\circ} \mathrm{C}$.

Synthesis of Biscycloadducts 45 and S3: To a 50-mL screw-cap tube containing a stir bar was added 44 ( $0.287 \mathrm{~g}, 0.749 \mathrm{mmol}, 1.0$ equiv.) and a crystal of BHT. The solids were dissolved in mesitylene ( $7.5 \mathrm{~mL})$,

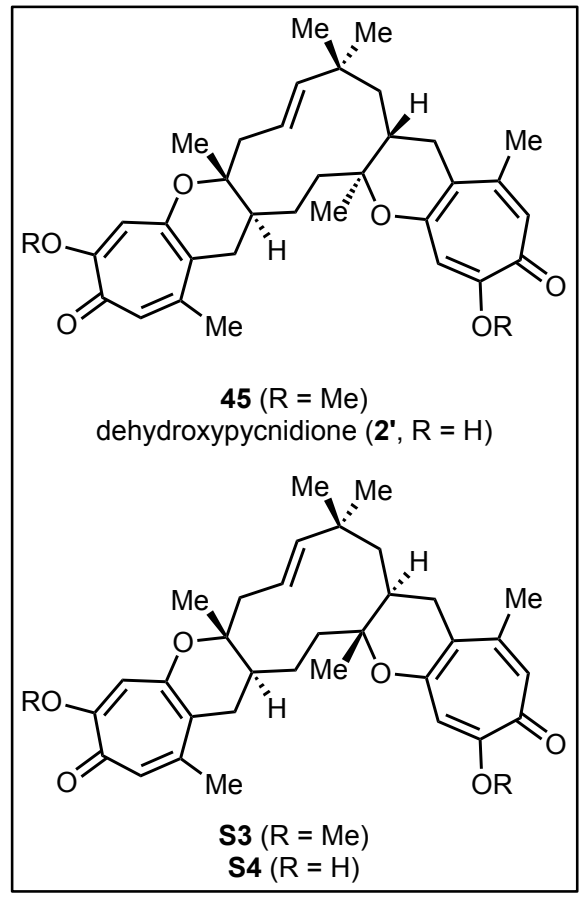
the tube was sealed with a septum cap, the septum was vented with two 18-gauge needles, and the vessel was placed in an aluminum block preheated to $160{ }^{\circ} \mathrm{C}$. A syringe pump was positioned next to the hotplate. Separately, a solution of tropolone $20(0.156 \mathrm{~g}, 0.749$ mmol, 1.0 equiv.) in ethanol $(8 \mathrm{~mL})$ was prepared. 20 does not readily dissolve in ethanol so sonication and gentle heating is required to achieve full dissolution. The solution of $\mathbf{2 0}$ was drawn into a $10-\mathrm{mL}$ Luer lock glass syringe with a Teflon-tipped plunger and using an 18-gauge metal needle, then the syringe was fitted into syringe pump with the needle positioned about three inches above the reaction level. It is important that this type of syringe is used with the ethanolic solution, as other syringe and solvent combinations cause the solution to leak. The solution of $\mathbf{2 0}$ was added dropwise to the reaction over about 16 hours, then the reaction was monitored by LCMS and TLC (2:1 EtOAc: $i-\mathrm{PrOH})$. About 24 hours after the addition of 20 was complete, it was determined to be fully consumed. At this time, the reaction was cooled to room 
temperature, then transferred to a recovery flask and concentrated by rotary evaporation. The crude reaction was purified by column chromatography (silica gel, EtOAc to 1:1 EtOAc:i-PrOH gradient) to give recovered $44(0.127 \mathrm{~g}, 0.332 \mathrm{mmol})$ and a mixture of $\mathbf{4 5}$ and $\mathbf{S 3}$ (1:1 ratio, $0.0544 \mathrm{~g}, 0.097 \mathrm{mmol}, 13 \%$, $23 \% \mathrm{brsm})$. The two isomers can be separated by preparative $\mathrm{HPLC}\left(\mathrm{C}_{18}, 50 \%\right.$ to $95 \% \mathrm{MeCN}$ in $\left.\mathrm{H}_{2} \mathrm{O}\right)$.

45

$\mathbf{R}_{\mathbf{f}} \quad 0.3\left(\mathrm{SiO}_{2}, \mathrm{EtOAc}: i-\mathrm{PrOH}=2: 1, \mathrm{UV}, \mathrm{KMnO}_{4}\right)$.

${ }^{1}$ H NMR $\quad\left(500 \mathrm{MHz}, \mathrm{CDCl}_{3}\right) \delta 7.02(\mathrm{~s}, 1 \mathrm{H}), 7.01(\mathrm{~s}, 1 \mathrm{H}), 6.47(\mathrm{~s}, 1 \mathrm{H}), 6.45(\mathrm{~s}, 1 \mathrm{H}), 5.56(\mathrm{~d}, J=15.7$ $\mathrm{Hz}, 1 \mathrm{H}), 5.47$ (ddd, $J=15.2,9.2,5.1 \mathrm{~Hz}, 1 \mathrm{H}), 3.91(\mathrm{~s}, 3 \mathrm{H}), 3.89(\mathrm{~s}, 3 \mathrm{H}), 2.75$ (dd, $J=17.2$, $5.3 \mathrm{~Hz}, 1 \mathrm{H}), 2.70-2.61(\mathrm{~m}, 2 \mathrm{H}), 2.42-2.35(\mathrm{~m}, 1 \mathrm{H}), 2.34(\mathrm{~s}, 3 \mathrm{H}), 2.29(\mathrm{~d}, J=1.0 \mathrm{~Hz}$, $3 \mathrm{H}), 2.28-2.22(\mathrm{~m}, 2 \mathrm{H}), 1.99(\mathrm{dd}, J=13.4,6.2 \mathrm{~Hz}, 1 \mathrm{H}), 1.91(\mathrm{~d}, J=13.5 \mathrm{~Hz}, 1 \mathrm{H}), 1.89$ $-1.83(\mathrm{~m}, 1 \mathrm{H}), 1.69-1.52(\mathrm{~m}, 3 \mathrm{H}), 1.49(\mathrm{dt}, J=11.7,6.2 \mathrm{~Hz}, 1 \mathrm{H}), 1.26(\mathrm{dt}, J=12.1,3.0$ $\mathrm{Hz}, 1 \mathrm{H}), 1.23(\mathrm{~s}, 3 \mathrm{H}), 1.13(\mathrm{~s}, 3 \mathrm{H}), 1.11(\mathrm{~s}, 3 \mathrm{H}), 1.08(\mathrm{~s}, 3 \mathrm{H})$.

${ }^{13}$ C NMR $\quad\left(126 \mathrm{MHz}, \mathrm{CDCl}_{3}\right) \delta 177.7177 .7,161.8,161.7,156.2,156.1,148.3,148.0,144.1,130.8$, $130.8,121.3,117.7,117.3,111.1,111.0,81.4,80.3,56.1,56.0,48.5,47.3,40.8,38.0,36.7$, $33.7,31.8,31.1,30.4,26.9,26.8,24.8,23.0,20.9,19.0$.

HRMS (ESI-TOF, m/z) calcd. for $\mathrm{C}_{35} \mathrm{H}_{45} \mathrm{O}_{6}[\mathrm{M}+\mathrm{H}]^{+}$calc.: 561.3216; found: 561.3203.

IR $\left(A T R\right.$, neat, $\left.\mathrm{cm}^{-1}\right) 2930(\mathrm{w}), 2851(\mathrm{w}), 1716(\mathrm{w}), 1600(\mathrm{~m}), 1562(\mathrm{~m}), 1481(\mathrm{~m}), 1377(\mathrm{w})$, $1182(\mathrm{~m}), 1140$ (m), 1010 (m), $984(\mathrm{~m}), 914$ (m), $879(\mathrm{~m}), 726$ (s), $643(\mathrm{~m})$.

mp $\quad 259-263{ }^{\circ} \mathrm{C}$.

S3

$\mathbf{R}_{\mathbf{f}} \quad 0.2\left(\mathrm{SiO}_{2}, \mathrm{EtOAc}: i-\mathrm{PrOH}=2: 1, \mathrm{UV}, \mathrm{KMnO}_{4}\right)$.

${ }^{1}$ H NMR $\quad\left(500 \mathrm{MHz}, \mathrm{CDCl}_{3}\right) \delta 7.08(\mathrm{~s}, 1 \mathrm{H}), 7.06(\mathrm{~s}, 1 \mathrm{H}), 6.50(\mathrm{~s}, 1 \mathrm{H}), 6.47(\mathrm{~s}, 1 \mathrm{H}), 5.86(\mathrm{~d}, J=15.9$ $\mathrm{Hz}, 1 \mathrm{H}$ ), 5.68 (ddd, $J=15.4,8.8,6.4 \mathrm{~Hz}, 1 \mathrm{H}), 3.94$ (s, 3H), 3.93 (s, 3H), 2.76 (dd, $J=17.6$, $5.6 \mathrm{~Hz}, 1 \mathrm{H}), 2.69-2.54(\mathrm{~m}, 3 \mathrm{H}), 2.41(\mathrm{~s}, 3 \mathrm{H}), 2.37-2.30(\mathrm{~m}, 1 \mathrm{H}) 2.34(\mathrm{~s}, 3 \mathrm{H}), 2.20-$ $2.13(\mathrm{~m}, 1 \mathrm{H}), 2.12-2.01(\mathrm{~m}, 1 \mathrm{H}), 1.98-1.90(\mathrm{~m}, 2 \mathrm{H}), 1.90-1.80(\mathrm{~m}, 2 \mathrm{H}), 1.69(\mathrm{~d}, J=$ $11.7 \mathrm{~Hz}, 1 \mathrm{H}), 1.62(\mathrm{~d}, J=14.0 \mathrm{~Hz}, 1 \mathrm{H}), 1.32(\mathrm{~s}, 3 \mathrm{H}), 1.23(\mathrm{~s}, 3 \mathrm{H}), 1.20-1.13(\mathrm{~m}, 1 \mathrm{H})$, $1.19(\mathrm{~s}, 3 \mathrm{H}), 1.16(\mathrm{~s}, 3 \mathrm{H})$.

${ }^{13}$ C NMR $\quad\left(126 \mathrm{MHz} \mathrm{CDCl}_{3}\right) \delta 177.6,177.6,161.8,161.7,156.3,156.1,148.3,148.1,144.6,130.8$, $130.8,121.2,118.1,117.5,111.4,110.8,80.8,80.0,56.0,56.0,45.8,36.6,36.0,33.7,33.6$, $30.6,30.4,30.1,26.9,26.7,26.7,21.1,20.9,19.4,19.0$.

HRMS (ESI-TOF, m/z) calcd. for $\mathrm{C}_{35} \mathrm{H}_{45} \mathrm{O}_{6}[\mathrm{M}+\mathrm{H}]^{+}$calc.: 561.3216; found: 561.3210.

IR (ATR, neat, $\left.\mathrm{cm}^{-1}\right) 2927(\mathrm{w}), 2844(\mathrm{w}), 1713(\mathrm{w}), 1601(\mathrm{~m}), 1560(\mathrm{~m}), 1481(\mathrm{~m}), 1180(\mathrm{~m})$, 1139 (m), 1004 (m), $914(\mathrm{~m}), 725$ (s), 641 (m).

mp $\quad 280^{\circ} \mathrm{C}($ decomp).

Synthesis of ( \pm )-Dehydroxypycnidione 2' from 45: To a 4-mL vial containing a stir bar was added 45 $(0.0033 \mathrm{~g}, 0.0059 \mathrm{mmol}, 1.0$ equiv. $)$ which was suspended in methanol $(0.20 \mathrm{~mL})$ and $1 \mathrm{M}$ sodium hydroxide ( $0.20 \mathrm{~mL}, 0.20 \mathrm{mmol}, 34$ equiv.) The suspension was stirred at $100^{\circ} \mathrm{C}$ and gradually became homogenous. 
The reaction was monitored by LCMS and the starting material was determined to be consumed after about 5 hours. The reaction was diluted with $\mathrm{pH} 7$ phosphate buffer, then $1 \mathrm{M}$ hydrochloric acid $(0.20 \mathrm{~mL})$ was added. The reaction was transferred to separatory funnel and extracted with ethyl acetate $(3 \times 1 \mathrm{~mL})$. The combined organic extracts were washed with brine, dried over magnesium sulfate, filtered, and concentrated to give $\mathbf{2}^{\prime}$ as an orange solid $(0.0024 \mathrm{~g}, 0.0045 \mathrm{mmol}, 77 \%)$.

$\mathbf{R}_{\mathbf{f}} \quad 0.3\left(\mathrm{SiO}_{2}, \mathrm{CH}_{2} \mathrm{Cl}_{2}: \mathrm{MeOH}=19: 1, \mathrm{UV}, \mathrm{KMnO}_{4}\right)$.

${ }^{1}$ H NMR $\quad\left(500 \mathrm{MHz}, \mathrm{CDCl}_{3}\right) \delta 7.13(\mathrm{~s}, 1 \mathrm{H}), 7.11(\mathrm{~s}, 1 \mathrm{H}), 6.97(\mathrm{~s}, 1 \mathrm{H}), 6.96(\mathrm{~s}, 1 \mathrm{H}), 5.52(\mathrm{~d}, J=15.6$ $\mathrm{Hz}, 1 \mathrm{H}), 5.44(\mathrm{ddd}, J=15.5,9.8,4.7 \mathrm{~Hz}, 1 \mathrm{H}), 2.77(\mathrm{dd}, J=17.1,5.2 \mathrm{~Hz}, 1 \mathrm{H}), 2.70-2.62$ $(\mathrm{m}, 2 \mathrm{H}), 2.44(\mathrm{~s}, 3 \mathrm{H}), 2.38(\mathrm{~s}, 3 \mathrm{H}), 2.36-2.24(\mathrm{~m}, 3 \mathrm{H}), 2.03-1.94(\mathrm{~m}, 2 \mathrm{H}), 1.87-1.76$ $(\mathrm{m}, 2 \mathrm{H}), 1.64(\mathrm{t}, J=13.0 \mathrm{~Hz}, 1 \mathrm{H}), 1.56(\mathrm{dd}, J=14.9,2.6 \mathrm{~Hz}, 1 \mathrm{H}), 1.47(\mathrm{dt}, J=11.9,5.7$ $\mathrm{Hz}, 1 \mathrm{H}), 1.36-1.27(\mathrm{~m}, 1 \mathrm{H}), 1.23(\mathrm{~s}, 3 \mathrm{H}), 1.13(\mathrm{~s}, 2 \mathrm{H}), 1.12(\mathrm{~s}, 3 \mathrm{H}), 1.10-1.08(\mathrm{~m}, 1 \mathrm{H})$, $1.07(\mathrm{~s}, 3 \mathrm{H})$.

${ }^{13} \mathbf{C}$ NMR $\quad\left(126 \mathrm{MHz} \mathrm{CDCl}_{3}\right) \delta 172.7,172.0,164.0,163.2,160.7,160.7,150.0,149.3,143.7,124.6$, $124.2,121.8,121.3,120.3,113.7,113.0,82.0,80.8,48.5,46.9,40.9,38.0,36.8,34.1,32.5$, $31.4,30.3,29.9,27.4,27.3,24.8,21.2,18.9$.

HRMS (ESI-TOF, m/z) calcd. for $\mathrm{C}_{33} \mathrm{H}_{41} \mathrm{O}_{6}[\mathrm{M}+\mathrm{H}]^{+}$calc.: 533.2903; found: 533.2900.

IR $\quad\left(A T R\right.$, neat, $\left.\mathrm{cm}^{-1}\right) 2922.5(\mathrm{~m}), 2851(\mathrm{w}), 1738(\mathrm{~s}), 1591(\mathrm{w}), 1439(\mathrm{~s}), 1375(\mathrm{~s}), 1285(\mathrm{~m})$, $1174(\mathrm{~s}), 891(\mathrm{~m}), 730(\mathrm{~m})$.

mp $\quad 230-235^{\circ} \mathrm{C}($ decomp).

Synthesis of ( \pm )-8,9-epi,epi-Dehydroxypycnidione S4 from S3: Employing a procedure that is identical to that used for the conversion of 45 to 2', $\mathbf{S 3}$ (0.0042 g, $0.0075 \mathrm{mmol}, 1.0$ equiv.) was hydrolyzed in methanol $(0.22 \mathrm{~mL})$ and $1 \mathrm{M}$ sodium hydroxide $(0.22 \mathrm{~mL}, 0.22 \mathrm{mmol}, 29$ equiv. $)$ at $100{ }^{\circ} \mathrm{C}$ to give $\mathbf{S 4}$ (0.0031 g, $0.0058 \mathrm{mmol}, 77 \%)$.

$\quad \mathbf{R}_{\mathbf{f}} \quad 0.3\left(\mathrm{SiO}_{2}, \mathrm{CH}_{2} \mathrm{Cl}_{2}: \mathrm{MeOH}=19: 1, \mathrm{UV}, \mathrm{KMnO}_{4}\right)$.

${ }^{1}$ H NMR $\quad\left(500 \mathrm{MHz}, \mathrm{CDCl}_{3}\right) \delta 7.13(\mathrm{~s}, 1 \mathrm{H}), 7.10(\mathrm{~s}, 1 \mathrm{H}), 6.94(\mathrm{~s}, 1 \mathrm{H}), 6.93(\mathrm{~s}, 1 \mathrm{H}), 5.72(\mathrm{~d}, J=15.9$ $\mathrm{Hz}, 1 \mathrm{H}), 5.61-5.53(\mathrm{~m}, 1 \mathrm{H}), 2.71(\mathrm{dd}, J=17.4,5.6 \mathrm{~Hz}, 1 \mathrm{H}), 2.62-2.51(\mathrm{~m}, 3 \mathrm{H}), 2.43(\mathrm{~s}$, $3 \mathrm{H}), 2.36(\mathrm{~s}, 3 \mathrm{H}), 2.12(\mathrm{~d}, J=11.2 \mathrm{~Hz}, 1 \mathrm{H}), 2.03-1.86(\mathrm{~m}, 3 \mathrm{H}), 1.83-1.69(\mathrm{~m}, 3 \mathrm{H}), 1.55$ $(\mathrm{d}, J=14.3 \mathrm{~Hz}, 1 \mathrm{H}), 1.37-1.26(\mathrm{~m}, 2 \mathrm{H}), 1.23(\mathrm{~s}, 3 \mathrm{H}), 1.15(\mathrm{~s}, 3 \mathrm{H}), 1.11(\mathrm{~s}, 3 \mathrm{H}), 1.10(\mathrm{~s}$, $3 \mathrm{H})$.

${ }^{13}$ C NMR $\quad\left(126 \mathrm{MHz}, \mathrm{CDCl}_{3}\right) \delta 172.8,172.4,163.2,160.7,160.7,150.5,149.7,144.5,133.6,133.5$, $124.8,124.5,121.1,113.5,113.2,81.3,80.4,45.5,36.3,33.9,32.1,31.4,30.1,29.9,29.5$, $27.4,27.2,23.8,22.8,21.7,19.5,14.3$.

HRMS (ESI-TOF, m/z) calcd. for $\mathrm{C}_{33} \mathrm{H}_{41} \mathrm{O}_{6}[\mathrm{M}+\mathrm{H}]^{+}$calc.: 533.2903; found: 533.2908.

IR (ATR, neat, $\left.\mathrm{cm}^{-1}\right) 3016(\mathrm{w}), 2970$ (s), 2924 (w), 1738 (s), 1693 (w), 1439 (m), 1366 (w), $1217(\mathrm{~m}), 894(\mathrm{w}), 723(\mathrm{w}), 528(\mathrm{w})$. 
Synthesis of Compounds 46 and 47: To a $35-\mathrm{mL}$ microwave vial was added methoxytropolone 20 (0.212 g, $1.02 \mathrm{mmol}, 1.0$ equiv.), alcohol 11 (2.49 g, $3.05 \mathrm{mmol}, 3.0$ equiv.), a crystal of BHT, and the mixture was suspended in mesitylene $(10.0 \mathrm{~mL}, 0.1 \mathrm{M})$. The vial was sealed and heated at $200{ }^{\circ} \mathrm{C}$ for 16 hours in a

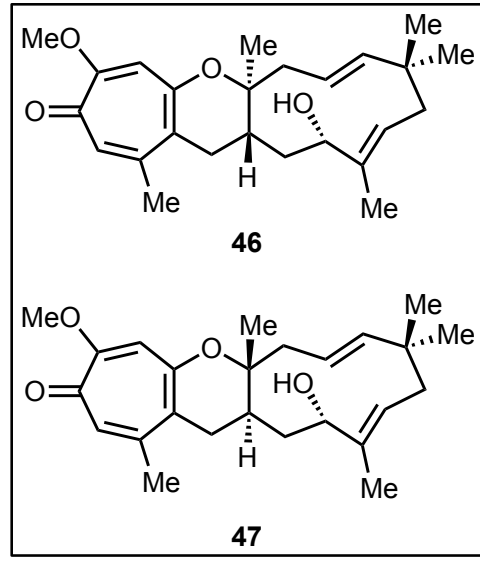

microwave reactor. Analysis by of the reaction by LCMS showed full consumption of $\mathbf{2 0}$ and formation of two products. The brown suspension was transferred to a recovery flask and concentrated under reduced pressure. The residue was then loaded onto silica gel and purified by flash column chromatography (silica gel, 3:1 hexanes:EtOAc to EtOAc to 7:1 EtOAc: $i-\mathrm{PrOH})$ to give recovered excess $\mathbf{1 1}$ and cycloadducts $\mathbf{4 6}$ and $\mathbf{4 7}$ as a 1.4:1 mixture (0.362 g, $0.908 \mathrm{mmol}, 89 \%)$. Compounds $\mathbf{4 6}$ and $\mathbf{4 7}$

were then separated using a Biotage Isolera One chromatography system (SNAP Ultra $\mathrm{C}_{18} 30 \mathrm{~g}$ column, 4:1 $\mathrm{H}_{2} \mathrm{O}: \mathrm{MeCN}$ to $1: 4 \mathrm{H}_{2} \mathrm{O}: \mathrm{MeCN}$ gradient).

46

$\mathbf{R}_{\mathbf{f}} \quad 0.4\left(\mathrm{SiO}_{2}, \mathrm{EtOAc}: i-\mathrm{PrOH}=9: 1, \mathrm{UV}, \mathrm{KMnO}_{4}\right)$.

${ }^{1}$ H NMR $\quad\left(500 \mathrm{MHz}, \mathrm{CDCl}_{3}\right) \delta 7.01(\mathrm{~s}, 1 \mathrm{H}), 6.50(\mathrm{~s}, 1 \mathrm{H}), 5.43(\mathrm{dd}, J=11.8,4.9 \mathrm{~Hz}, 1 \mathrm{H}), 5.16(\mathrm{dd}, J$ $=16.0,1.5 \mathrm{~Hz}, 1 \mathrm{H}), 5.09(\mathrm{ddd}, J=16.0,9.6,1.9 \mathrm{~Hz}, 1 \mathrm{H}), 4.35(\mathrm{~d}, J=6.7 \mathrm{~Hz}, 1 \mathrm{H}), 3.89(\mathrm{~s}$, $3 \mathrm{H}), 3.16(\mathrm{dd}, J=17.7,5.4 \mathrm{~Hz}, 1 \mathrm{H}), 2.56(\mathrm{~d}, J=14.8 \mathrm{~Hz}, 1 \mathrm{H}), 2.30(\mathrm{~s}, 3 \mathrm{H}), 2.23-2.15$ $(\mathrm{m}, 3 \mathrm{H}), 2.00(\mathrm{dt}, J=12.0,6.0 \mathrm{~Hz}, 1 \mathrm{H}), 1.84(\mathrm{dd}, J=12.4,4.8 \mathrm{~Hz}, 1 \mathrm{H}), 1.68(\mathrm{~d}, J=4.4$ $\mathrm{Hz}, 1 \mathrm{H}), 1.54$ (s, 3H), $1.58-1.46(\mathrm{~m}, 2 \mathrm{H}), 1.09$ (s, 3H), 1.09 (s, 3H), 1.07 (s, 3H).

${ }^{13}$ C NMR $\quad\left(126 \mathrm{MHz}, \mathrm{CDCl}_{3}\right) \delta 177.6,161.6,156.5,148.8,141.8,139.5,130.6,120.9,120.6,118.7$, $111.4,81.4,73.6,56.0,42.5,40.9,38.9,34.8,33.8,30.2,29.1,26.9,24.2,20.4,16.5$.

HRMS (ESI-TOF, m/z) calcd. for $\mathrm{C}_{25} \mathrm{H}_{35} \mathrm{O}_{4}[\mathrm{M}+\mathrm{H}]^{+}$calc.: 399.2535; found: 399.2522.

IR (ATR, neat, $\left.\mathrm{cm}^{-1}\right) 3369(\mathrm{br}), 2935(\mathrm{w}), 1599(\mathrm{~m}), 1547(\mathrm{~m}), 1480(\mathrm{~m}), 1272(\mathrm{~m}), 1179(\mathrm{~s})$, 1132 (s), $1011(\mathrm{~s}), 881(\mathrm{~m}), 726(\mathrm{~s}), 643(\mathrm{~m}), 564(\mathrm{~m})$.

mp $\quad 148-152{ }^{\circ} \mathrm{C}$.

$[\alpha]_{\mathbf{D}}{ }^{23}+130.0\left(\mathrm{c}=1.0\right.$ in $\left.\mathrm{CHCl}_{3}\right)$.

47

$\mathbf{R}_{\mathbf{f}} \quad 0.4\left(\mathrm{SiO}_{2}, \mathrm{EtOAc}: i-\mathrm{PrOH}=9: 1, \mathrm{UV}, \mathrm{KMnO}_{4}\right)$.

${ }^{1}$ H NMR $\quad\left(500 \mathrm{MHz}, \mathrm{CDCl}_{3}\right) \delta 7.01(\mathrm{~s}, 1 \mathrm{H}), 6.48(\mathrm{~s}, 1 \mathrm{H}), 5.22-5.16(\mathrm{~m}, 2 \mathrm{H}), 5.06(\mathrm{ddd}, J=15.9$, $10.4,2.5 \mathrm{~Hz}, 1 \mathrm{H}), 3.96(\mathrm{~d}, J=9.9 \mathrm{~Hz}, 1 \mathrm{H}), 3.89(\mathrm{~s}, 3 \mathrm{H}), 2.86(\mathrm{dd}, J=17.6,5.4 \mathrm{~Hz}, 1 \mathrm{H})$, $2.56(\mathrm{dt}, J=14.7,2.3 \mathrm{~Hz}, 1 \mathrm{H}), 2.31(\mathrm{~s}, 3 \mathrm{H}), 2.32-2.20(\mathrm{~m}, 4 \mathrm{H}), 1.79(\mathrm{dd}, J=12.8,4.7$ 
$\mathrm{Hz}, 1 \mathrm{H}), 1.74$ (dd, $J=13.6,9.9 \mathrm{~Hz}, 1 \mathrm{H}), 1.65(\mathrm{~s}, 3 \mathrm{H}), 1.16-1.10(\mathrm{~m}, 1 \mathrm{H}), 1.10(\mathrm{~s}, 3 \mathrm{H})$, $1.07(\mathrm{~s}, 3 \mathrm{H}), 1.03(\mathrm{~s}, 3 \mathrm{H})$.

${ }^{13} \mathbf{C ~ N M R ~} \quad\left(126 \mathrm{MHz} \mathrm{CDCl}_{3}\right) \delta 177.6,161.7,156.7,148.5,142.3,138.7,130.6,123.7,120.5,117.2$, $111.2,81.0,78.1,56.0,42.6,40.7,39.0,38.8,33.6,30.9,30.3,26.9,24.2,20.1,10.7$.

HRMS (ESI-TOF, m/z) calcd. for $\mathrm{C}_{25} \mathrm{H}_{35} \mathrm{O}_{4}[\mathrm{M}+\mathrm{H}]^{+}$calc.: 399.2535; found: 399.2522.

IR (ATR, neat, $\left.\mathrm{cm}^{-1}\right) 3366(\mathrm{br}), 2941(\mathrm{w}), 1599(\mathrm{~m}), 1548(\mathrm{~m}), 1478(\mathrm{~m}), 1178(\mathrm{~s}), 1134(\mathrm{~s})$, $1009(\mathrm{~s}), 879(\mathrm{~m}), 727(\mathrm{~s}), 642(\mathrm{~m}), 552(\mathrm{~m})$.

mp $\quad 156-160^{\circ} \mathrm{C}$.

$[\boldsymbol{\alpha}]_{\mathbf{D}}{ }^{23} \quad-121.4\left(\mathrm{c}=1.00\right.$ in $\left.\mathrm{CHCl}_{3}\right)$.

Synthesis of Carbamate 48: To a 4-mL vial containing a solution of $47(0.0200 \mathrm{~g}, 0.0502 \mathrm{mmol}, 1.0$

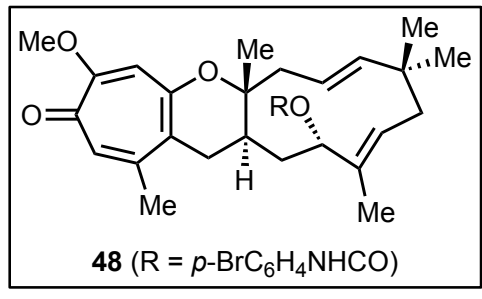

equiv.) in anhydrous dichloromethane $(0.50 \mathrm{~mL}, 0.1 \mathrm{M})$ was added triethylamine $(0.014 \mathrm{~mL}, 0.0102 \mathrm{~g}, 0.100 \mathrm{mmol}, 2.0$ equiv.) followed by p-bromophenyl isocyanate $(0.0149 \mathrm{~g}, 0.0753 \mathrm{mmol}, 1.5$ equiv.). The reaction gradually became a yellow suspension and stirring was continued

overnight. The starting material was determined to be consumed in exchange for two products by TLC (EtOAc). The reaction was quenched with saturated aqueous ammonium chloride $(1 \mathrm{~mL})$, then the mixture was transferred to a separatory funnel and the vial was rinsed with ethyl acetate $(1 \mathrm{~mL})$. The layers were separated, and the aqueous layer was washed with ethyl acetate $(3 \times 1 \mathrm{~mL})$. The combined organic extracts were washed with brine $(2 \mathrm{~mL})$, dried over magnesium sulfate, filtered, and concentrated. The crude material was purified by column chromatography (silica gel, EtOAc) to give 48 (0.0216 g, $0.0362 \mathrm{mmol}$, $72 \%$ ). The two products were separated by prep-TLC (silica gel, EtOAc), and the major, later eluting product was recrystallized from methanol to produce crystals suitable for X-ray diffraction. Through single crystal X-ray analysis, the structure was unambiguously proven.

$\mathbf{R}_{\mathbf{f}} \quad 0.3\left(\mathrm{SiO}_{2}, \mathrm{EtOAc}, \mathrm{UV}, \mathrm{KMnO}_{4}\right)$.

${ }^{1}$ H NMR $\quad\left(500 \mathrm{MHz}, \mathrm{CDCl}_{3}\right) \delta 7.41(\mathrm{~d}, J=8.0 \mathrm{~Hz}, 2 \mathrm{H}), 7.29(\mathrm{~d}, J=8.0 \mathrm{~Hz}, 2 \mathrm{H}), 7.04(\mathrm{~s}, 1 \mathrm{H}), 6.65$ $(\mathrm{s}, 1 \mathrm{H}), 6.49(\mathrm{~s}, 1 \mathrm{H}), 5.33(\mathrm{dd}, J=11.9,3.6 \mathrm{~Hz}, 1 \mathrm{H}), 5.19$ (d, $J=15.9 \mathrm{~Hz}, 1 \mathrm{H}), 5.11$ (ddd, $J=15.9,10.0,2.0 \mathrm{~Hz}, 1 \mathrm{H}), 4.88(\mathrm{~d}, J=9.9 \mathrm{~Hz}, 1 \mathrm{H}), 3.90$ (s, 3H), 3.14 (dd, $J=17.9,5.4$ $\mathrm{Hz}, 1 \mathrm{H}), 2.59$ (d, $J=14.8 \mathrm{~Hz}, 1 \mathrm{H}), 2.37$ (s, 3H), 2.29 (dd, $J=15.8,10.8 \mathrm{~Hz}, 1 \mathrm{H}), 2.24$ (dd, $J=11.7,8.4 \mathrm{~Hz}, 1 \mathrm{H}), 1.85(\mathrm{dd}, J=13.0,4.6 \mathrm{~Hz}, 1 \mathrm{H}), 1.81-1.72(\mathrm{~m}, 2 \mathrm{H}), 1.67(\mathrm{~s}, 3 \mathrm{H})$, $1.29-1.22(\mathrm{~m}, 2 \mathrm{H}), 1.10(\mathrm{~s}, 3 \mathrm{H}), 1.09(\mathrm{~s}, 3 \mathrm{H}), 1.07(\mathrm{~s}, 3 \mathrm{H})$. 
${ }^{13} \mathbf{C}$ NMR $\quad\left(126 \mathrm{MHz} \mathrm{CDCl}_{3}\right) \delta 177.7,161.7,156.4,152.6,148.7,142.5,137.1,134.8,132.2,130.8$ $125.4,120.5,120.2,117.3,116.1,111.2,80.6,56.0,42.7,40.6,38.6,37.0,33.7,30.5,30.4$, $29.8,26.9,24.3,20.1,11.6$.

HRMS (ESI-TOF, m/z) calcd. for $\mathrm{C}_{32} \mathrm{H}_{39} \mathrm{BrNO}_{5}[\mathrm{M}+\mathrm{H}]^{+}$calc.: 596.2012; found: 596.2005.

IR $\quad\left(\right.$ ATR, neat, $\left.\mathrm{cm}^{-1}\right) 3366$ (br), $2922(\mathrm{~m}), 1721(\mathrm{~m}), 1598(\mathrm{~s}), 1538(\mathrm{~s}), 1474$ (s), 1398 (s), $1308(\mathrm{~m}), 1276(\mathrm{~m}), 1178(\mathrm{~s}), 1136(\mathrm{~s}), 1012(\mathrm{~s}), 824(\mathrm{~m}), 504(\mathrm{~s})$. 1480 (m), 1272 (m), 1179 (s), 1132 (s), 1011 (s), 881 (m), 726 (s), 643 (m), 564 (m).

mp $\quad 220-223^{\circ} \mathrm{C}$.

$[\alpha]_{\mathbf{D}}{ }^{23}-83.0\left(\mathrm{c}=0.46\right.$ in $\left.\mathrm{CHCl}_{3}\right)$.

Synthesis of (+)-Isoepolone B (ent-16): To a 4-mL vial containing compound 46 (0.0100 g, $0.025 \mathrm{mmol}$,

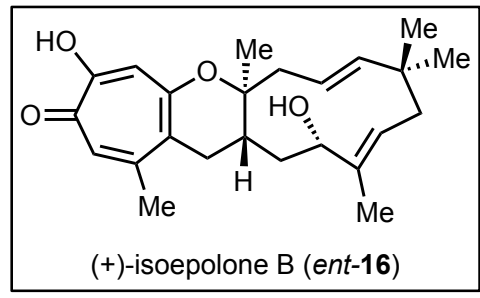

1.0 equiv.) was added methanol $(0.25 \mathrm{~mL})$ and $1 \mathrm{M}$ sodium hydroxide (0.25 mL, $0.25 \mathrm{mmol}, 10.0$ equiv.). The suspension was heated to $100{ }^{\circ} \mathrm{C}$ and vigorously stirred, eventually becoming homogenous. Consumption of the starting material was observed after six hours, then the reaction was

transferred to a separatory funnel containing $\mathrm{pH}=7$ phosphate buffer $(1 \mathrm{~mL})$ and rinsed with ethyl acetate $(1 \mathrm{~mL}) .1 \mathrm{M}$ Hydrochloric acid $(0.25 \mathrm{~mL})$ was added to the separatory funnel to neutralize residual sodium hydroxide. The $\mathrm{pH}$ of the aqueous layer was measured and determined to be about $\mathrm{pH}=3$. The aqueous layer was extracted with ethyl acetate $(3 \times 2 \mathrm{~mL})$, then the combined organic extracts were washed with brine $(2 \mathrm{~mL})$, dried over magnesium sulfate, filtered, and concentrated under reduced pressure to give ent16 as an orange solid $(0.0095 \mathrm{~g}, 0.025 \mathrm{mmol}, 99 \%$.).

$\mathbf{R}_{\mathbf{f}} \quad 0.4\left(\mathrm{SiO}_{2}, \mathrm{CH}_{2} \mathrm{Cl}_{2}=19: 1, \mathrm{UV}, \mathrm{KMnO}_{4}\right)$.

${ }^{1}$ H NMR $\quad\left(500 \mathrm{MHz}, \mathrm{CDCl}_{3}\right) \delta 7.11(\mathrm{~s}, 1 \mathrm{H}), 7.00(\mathrm{~s}, 1 \mathrm{H}), 5.42(\mathrm{dd}, J=11.7,5.1 \mathrm{~Hz}, 1 \mathrm{H}), 5.16(\mathrm{dd}, J$ $=15.9,1.8 \mathrm{~Hz}, 1 \mathrm{H}), 5.07$ (ddd, $J=15.9,9.8,2.4 \mathrm{~Hz}, 1 \mathrm{H}), 4.36(\mathrm{~d}, J=6.6 \mathrm{~Hz}, 1 \mathrm{H}), 3.23$ $(\mathrm{dd}, J=17.6,5.3 \mathrm{~Hz}, 1 \mathrm{H}), 2.58(\mathrm{dt}, J=14.8,2.1 \mathrm{~Hz}, 1 \mathrm{H}), 2.39(\mathrm{~s}, 3 \mathrm{H}), 2.26(\mathrm{dd}, J=17.7$, $11.8 \mathrm{~Hz}, 1 \mathrm{H}), 2.23-2.16(\mathrm{~m}, 2 \mathrm{H}), 2.02(\mathrm{dt}, J=12.0,6.1 \mathrm{~Hz}, 1 \mathrm{H}), 1.85(\mathrm{dd}, J=12.6,5.1$ $\mathrm{Hz}, 1 \mathrm{H}), 1.56-1.52(\mathrm{~m}, 1 \mathrm{H}), 1.55(\mathrm{~s}, 3 \mathrm{H}), 1.50(\mathrm{~d}, J=14.2 \mathrm{~Hz}, 1 \mathrm{H}), 1.12(\mathrm{~s}, 3 \mathrm{H}), 1.09$ (s, $3 \mathrm{H}), 1.04(\mathrm{~s}, 3 \mathrm{H})$.

${ }^{13}$ C NMR $\quad\left(126 \mathrm{MHz}, \mathrm{CDCl}_{3}\right) \delta 172.4,163.4,161.2,150.1,142.0,139.4,124.4,122.0,120.9,120.8$, $113.5,82.1,73.8,42.8,40.9,38.9,34.7,34.2,30.0,29.3,27.4,24.3,20.6,16.5$.

HRMS EI-TOF, m/z) calcd. for $\mathrm{C}_{24} \mathrm{H}_{32} \mathrm{O}_{4}[\mathrm{M}]^{+}$calc.: 384.2301; found: 384.2320 .

IR $\quad\left(\right.$ ATR, neat, $\left.\mathrm{cm}^{-1}\right) 3365(\mathrm{br}), 3187(\mathrm{br}), 2932(\mathrm{w}), 2861(\mathrm{w}), 1591(\mathrm{~m}), 1525(\mathrm{w}), 1443(\mathrm{~s})$, $1375(\mathrm{~m}), 1280(\mathrm{~m}), 1176(\mathrm{~s}), 1131(\mathrm{~s}), 1092$ (s), $1031(\mathrm{~m}), 983(\mathrm{~m}), 891(\mathrm{~m}), 729(\mathrm{~s}), 568$ (w), 469 (w). 
mp $\quad 107-109^{\circ} \mathrm{C}$.

$[\alpha]_{\mathbf{D}}{ }^{23}+65.8\left(\mathrm{c}=1.00\right.$ in $\left.\mathrm{CHCl}_{3}\right)$.

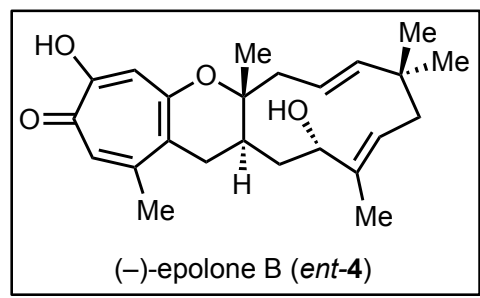

Synthesis of (-)-Epolone B (ent-4): Employing a procedure identical to that which converted 46 to (+)-isoepolone B (16), $47(0.0103 \mathrm{~g}, 0.0258$ mmol, 1.0 equiv. $)$ was heated to $100{ }^{\circ} \mathrm{C}$ in $1 \mathrm{M}$ sodium hydroxide $(0.26$ $\mathrm{mL}, 0.26 \mathrm{mmol}, 10.0$ equiv. $)$ in methanol $(0.26 \mathrm{~mL})$ to give ent -4 as an

orange solid (0.0099 g, $0.0285 \mathrm{mmol}, 99 \%)$.

$\mathbf{R}_{\mathbf{f}}$

$0.3\left(\mathrm{SiO}_{2}, \mathrm{CH}_{2} \mathrm{Cl}_{2}=19: 1, \mathrm{UV}, \mathrm{KMnO}_{4}\right)$.

${ }^{1}$ H NMR $\quad\left(500 \mathrm{MHz}, \mathrm{CDCl}_{3}\right) \delta 7.12(\mathrm{~s}, 1 \mathrm{H}), 6.99(\mathrm{~s}, 1 \mathrm{H}), 5.23-5.15(\mathrm{~m}, 2 \mathrm{H}), 5.05(\mathrm{ddd}, J=15.9$, $10.4,2.6 \mathrm{~Hz}, 1 \mathrm{H}), 3.97(\mathrm{~d}, J=9.8 \mathrm{~Hz}, 1 \mathrm{H}), 2.90(\mathrm{dd}, J=17.0,4.0 \mathrm{~Hz}, 1 \mathrm{H}), 2.58(\mathrm{~d}, J=$ $14.8 \mathrm{~Hz}, 1 \mathrm{H}), 2.40(\mathrm{~s}, 3 \mathrm{H}), 2.39-2.33(\mathrm{~m}, 1 \mathrm{H}), 2.28-2.22(\mathrm{~m}, 2 \mathrm{H}), 1.82-1.72(\mathrm{~m}, J=$ 23.1, 13.1, 7.2 Hz, 2H), $1.70-1.66(\mathrm{~m}, 1 \mathrm{H}), 1.65(\mathrm{~s}, 3 \mathrm{H}), 1.18-1.14(\mathrm{~m}, 1 \mathrm{H}), 1.12(\mathrm{~s}$, $3 \mathrm{H}), 1.07(\mathrm{~s}, 3 \mathrm{H}), 1.01(\mathrm{~s}, 3 \mathrm{H})$.

${ }^{13}$ C NMR $\quad\left(126 \mathrm{MHz}, \mathrm{CDCl}_{3}\right) \delta 172.8,163.1,161.1,150.1,142.3,138.5,124.6,124.0,120.4,120.2$, $113.3,81.5,78.1,42.7,40.8,38.8,38.8,33.6,31.5,30.2,27.5,24.2,20.3,10.8$.

HRMS (EI-TOF, m/z) calcd. for $\mathrm{C}_{24} \mathrm{H}_{32} \mathrm{O}_{4}[\mathrm{M}]^{+}$calc.: 384.23006; found: 384.23107.

IR $\left(\right.$ ATR, neat, $\left.\mathrm{cm}^{-1}\right) 3377(\mathrm{br}), 3195(\mathrm{br}), 2935(\mathrm{~m}), 2862(\mathrm{w}), 1590(\mathrm{~m}), 1443(\mathrm{~s}), 1376(\mathrm{~m})$, 1233 (m), 1159 (s), 1135 (m), 1051 (m), 1001 (m), 754 (m).

mp $\quad 169-173^{\circ} \mathrm{C}$.

$[\boldsymbol{\alpha}]_{\mathbf{D}}{ }^{23}-84.0\left(\mathrm{c}=0.35\right.$ in $\left.\mathrm{CHCl}_{3}\right)$.

Synthesis of Biscycloadduct 51: To a 50-mL screw-cap tube was added 46 ( $0.249 \mathrm{~g}, 0.624 \mathrm{mmol}, 1.0$

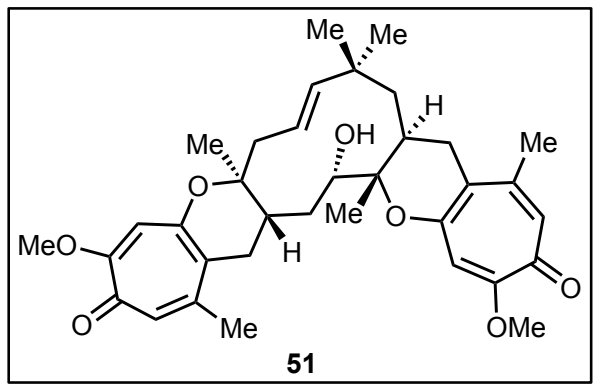
equiv.) and a crystal of BHT. The solids were dissolved in mesitylene $(7.5 \mathrm{~mL})$, the tube was sealed with a septum cap, the septum was vented with two 18-gauge needles, and the vessel was placed in an aluminum block preheated to $160^{\circ} \mathrm{C}$. A syringe pump was positioned next to the hotplate. Separately, a solution of tropolone $(0.130 \mathrm{~g}, 0.624 \mathrm{mmol}, 1.0$ equiv. $)$ in ethanol $(8 \mathrm{~mL})$ was prepared. $\mathbf{2 0}$ does not readily dissolve in ethanol so sonication and gentle heating is required to achieve full dissolution. The solution of $\mathbf{2 0}$ was drawn into a 10 -mL Luer lock glass syringe with a Teflon-tipped plunger and using an 18-gauge metal needle, then the syringe was fitted into syringe pump with the needle positioned about three inches above 
the reaction level. It is important that this type of syringe is used with the ethanolic solution, as other syringe and solvent combinations cause the solution to leak. The solution of $\mathbf{2 0}$ was added dropwise to the reaction over about 16 hours, then the reaction was monitored by LCMS and TLC (2:1 EtOAc:i-PrOH). About 24 hours after the addition of $\mathbf{2 0}$ was complete, it was determined to be fully consumed. At this time, the reaction was cooled to room temperature, then transferred to a recovery flask and concentrated by rotary evaporation. The crude reaction was purified by column chromatography (silica gel, EtOAc to 1:1 EtOAc:iPrOH gradient) to give recovered $46(0.120 \mathrm{~g}, 0.301 \mathrm{mmol})$ and $\mathbf{5 1}(0.0847 \mathrm{~g}, 0.147 \mathrm{mmol}, 24 \%, 46 \%$ brsm).

${ }^{1}$ H NMR $\quad\left(500 \mathrm{MHz}, \mathrm{CDCl}_{3}\right) \delta 7.01(\mathrm{~s}, 1 \mathrm{H}), 6.92(\mathrm{~s}, 1 \mathrm{H}), 6.43(\mathrm{~s}, 1 \mathrm{H}), 6.41(\mathrm{~s}, 1 \mathrm{H}), 5.74(\mathrm{~d}, J=15.7$ $\mathrm{Hz}, 1 \mathrm{H}), 5.35(\mathrm{ddd}, J=15.4,10.5,4.3 \mathrm{~Hz}, 1 \mathrm{H}), 4.94(\mathrm{~s}, 1 \mathrm{H}), 4.13(\mathrm{dt}, J=11.4,5.4 \mathrm{~Hz}$, $1 \mathrm{H}), 3.94(\mathrm{~s}, 3 \mathrm{H}), 3.91(\mathrm{~s}, 3 \mathrm{H}), 2.89(\mathrm{t}, J=13.1 \mathrm{~Hz}, 1 \mathrm{H}), 2.72-2.63(\mathrm{~m}, 3 \mathrm{H}), 2.59(\mathrm{dd}, J$ $=17.5,5.7 \mathrm{~Hz}, 1 \mathrm{H}), 2.38(\mathrm{t}, J,=11.7 \mathrm{~Hz}, 1 \mathrm{H}), 2.34-2.36(\mathrm{~m}, 2 \mathrm{H}), 2.31(\mathrm{~s}, 3 \mathrm{H}), 2.30(\mathrm{~s}$, $3 \mathrm{H}), 2.13-2.03(\mathrm{~m}, 2 \mathrm{H}), 1.96(\mathrm{dt}, J=9.4,4.2 \mathrm{~Hz}, 1 \mathrm{H}), 1.42(\mathrm{~s}, 3 \mathrm{H}), 1.15(\mathrm{~s}, 3 \mathrm{H}), 1.14(\mathrm{~s}$, $3 \mathrm{H}), 1.06(\mathrm{~s}, 3 \mathrm{H}), 0.90(\mathrm{dd}, J=14.6,4.2 \mathrm{~Hz}, 1 \mathrm{H})$.

${ }^{13}$ C NMR $\quad\left(126 \mathrm{MHz} \mathrm{CDCl}_{3}\right) \delta 177.7,176.8,161.7,161.1,156.7,155.8,149.7,148.8,148.4,130.7$, 129.6, 120.6, 118.5, 117.8, 111.6, 111.1, 82.1, 81.0, 75.1, 56.2, 56.1, 49.2, 45.2, 37.4, 34.9, $33.1,31.8,31.5,31.4,30.4,27.2,26.7,21.8,19.4,18.7$.

HRMS (ESI-TOF, m/z) calcd. for $\mathrm{C}_{35} \mathrm{H}_{45} \mathrm{O}_{7}[\mathrm{M}+\mathrm{H}]^{+}$calc.: 577.3165; found: 577.3151.

IR (ATR, neat, $\left.\mathrm{cm}^{-1}\right) 3348(\mathrm{br}), 2927(\mathrm{w}), 2855(\mathrm{w}), 1715(\mathrm{w}), 1599(\mathrm{~m}), 1547(\mathrm{~m}), 1478(\mathrm{~m})$, 1455 (m), 1375 (w), 1184 (s), 1152 (s), 1068, 1011 (m), 879 (m), 733 (w), 666 (w).

mp $\quad 238-240{ }^{\circ} \mathrm{C}$.

$[\alpha]_{\mathbf{D}}{ }^{23}+160.0\left(\mathrm{c}=0.47\right.$ in $\left.\mathrm{CHCl}_{3}\right)$.

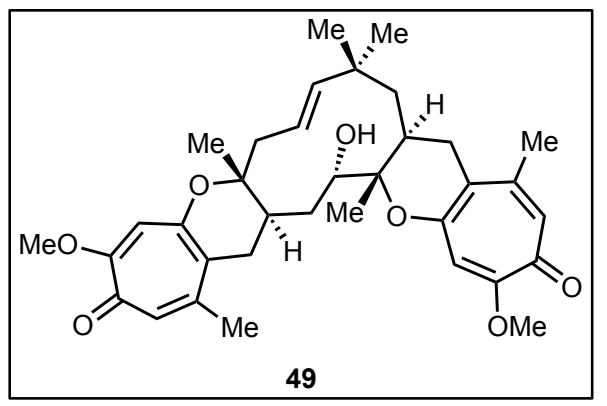

Synthesis of Biscycloadduct 49: Employing a procedure identical to that used for the preparation and purification of biscycloadduct 51, 47 (0.172 g, $0.432 \mathrm{mmol}, 1.0$ equiv.) was reacted with tropolone $\mathbf{2 0}(0.0900,0.432 \mathrm{mmol}, 1.0$ equiv. $)$ to give recovered $47(0.0853 \mathrm{~g}, 0.214 \mathrm{mmol})$ and $49(0.0655 \mathrm{~g}, 0.114$

mmol, $26 \%, 52 \%$ brsm).

$\mathbf{R}_{\mathbf{f}}$

${ }^{1}$ H NMR
$0.2\left(\mathrm{SiO}_{2}, \mathrm{EtOAc}: i-\mathrm{PrOH}=2: 1, \mathrm{UV}, \mathrm{KMnO}_{4}\right)$.

$\left(500 \mathrm{MHz}, \mathrm{CDCl}_{3}\right) \delta 7.10(\mathrm{~s}, 1 \mathrm{H}), 7.03(\mathrm{~s}, 1 \mathrm{H}), 6.49(\mathrm{~s}, 1 \mathrm{H}), 6.44(\mathrm{~s}, 1 \mathrm{H}), 5.49$ (ddd, $J=$ $13.8,7.9,5.6 \mathrm{~Hz}, 1 \mathrm{H}), 5.43(\mathrm{~d}, J=15.8 \mathrm{~Hz}, 1 \mathrm{H}), 3.91(\mathrm{~s}, 3 \mathrm{H}), 3.89(\mathrm{~s}, 3 \mathrm{H}), 3.71(\mathrm{t}, J=4.0$ $\mathrm{Hz}, 1 \mathrm{H}), 2.98-2.90(\mathrm{~m}, 2 \mathrm{H}), 2.65(\mathrm{dd}, J=14.2,5.4 \mathrm{~Hz}, 1 \mathrm{H}), 2.53-2.43$ (m, 3H), $2.38-$ 
$2.26(\mathrm{~m}, 1 \mathrm{H}), 2.33(\mathrm{~s}, 3 \mathrm{H}), 2.31(\mathrm{~s}, 3 \mathrm{H}), 2.19-2.11(\mathrm{~m}, 1 \mathrm{H}), 1.90$ (dddd, $J=25.9,12.5$, 6.0, 3.2 Hz, 1H), $1.57(\mathrm{~d}, J=15.1 \mathrm{~Hz}, 1 \mathrm{H}), 1.17(\mathrm{~s}, 3 \mathrm{H}), 1.16(\mathrm{~s}, 3 \mathrm{H}), 1.09(\mathrm{~s}, 3 \mathrm{H}), 1.08(\mathrm{~s}$, $3 \mathrm{H}), 1.07-1.03(\mathrm{~m}, 1 \mathrm{H}), 0.93(\mathrm{dd}, J=14.8,5.2 \mathrm{~Hz}, 1 \mathrm{H})$.

${ }^{13} \mathbf{C ~ N M R ~} \quad\left(126 \mathrm{MHz}, \mathrm{CDCl}_{3}\right) \delta 177.7,177.7,162.6,161.7,156.3,156.3,148.5,146.6,143.5,132.6$, $130.9,122.7,117.5,117.5,111.2,110.7,87.6,79.8,78.4,56.3,56.0,45.8,44.1,40.1,37.7$, $36.3,33.0,32.9,30.7,29.8,29.4,26.8,24.0,18.8,16.1$.

HRMS (ESI-TOF, m/z) calcd. for $\mathrm{C}_{35} \mathrm{H}_{45} \mathrm{O}_{7}[\mathrm{M}+\mathrm{H}]^{+}$calc.: 577.3165; found: 577.3152 .

IR $\left(\right.$ ATR, neat, $\left.\mathrm{cm}^{-1}\right) 3410(\mathrm{br}), 2960(\mathrm{~m}), 2931(\mathrm{~m}), 2869(\mathrm{~m}), 1715(\mathrm{w}), 1601(\mathrm{~s}), 1556(\mathrm{~s})$, $1483(\mathrm{~s}), 1430(\mathrm{~m}), 1266(\mathrm{~m}), 1183(\mathrm{~s}), 1151$ (s), $1009(\mathrm{~m}), 881(\mathrm{w}), 750(\mathrm{~s}), 662(\mathrm{w})$.

mp $\quad 204-206^{\circ} \mathrm{C}$

$[\alpha]_{\mathbf{D}}{ }^{23}+33.0\left(\mathrm{c}=0.49\right.$ in $\left.\mathrm{CHCl}_{3}\right)$.

Synthesis of 10-Epi-(-)-Pycnidione (52): To a 4-mL vial was added 51 (0.010 g, 0.0173 mmol. 1.0 equiv.)

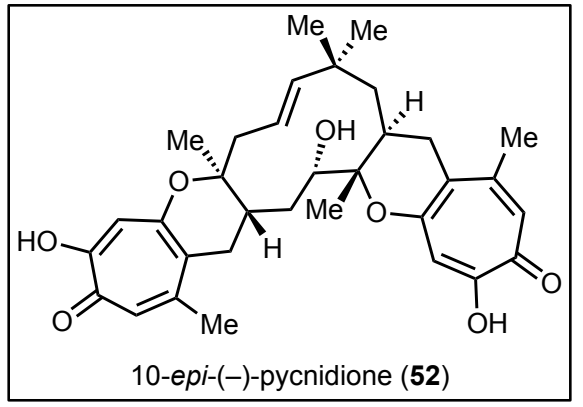

buffer, then $1 \mathrm{M}$ hydrochloric acid $(0.50 \mathrm{~mL})$ was added. The reaction was transferred to separatory funnel and extracted with ethyl acetate $(3 \times 1 \mathrm{~mL})$. The combined organic extracts were washed with brine, dried over magnesium sulfate, filtered, and concentrated to give $\mathbf{5 2}$ as an orange solid $(0.0077 \mathrm{~g}, 0.014 \mathrm{mmol}$, $81 \%)$.

$\mathbf{R}_{\mathbf{f}} \quad 0.2\left(\mathrm{SiO}_{2}, \mathrm{CH}_{2} \mathrm{Cl}_{2}: \mathrm{MeOH}=19: 1, \mathrm{UV}, \mathrm{KMnO}_{4}\right)$.

${ }^{1}$ H NMR $\quad\left(500 \mathrm{MHz}, \mathrm{CD}_{2} \mathrm{Cl}_{2}\right) \delta 7.07(\mathrm{~s}, 1 \mathrm{H}), 7.03(\mathrm{~s}, 1 \mathrm{H}), 6.91(\mathrm{~s}, 1 \mathrm{H}), 6.90(\mathrm{~s}, 1 \mathrm{H}), 5.67(\mathrm{~d}, J=15.8$ $\mathrm{Hz}, 1 \mathrm{H}), 5.40(\mathrm{ddd}, J=15.5,10.5,4.5 \mathrm{~Hz}, 1 \mathrm{H}), 4.02-3.96(\mathrm{~m}, 1 \mathrm{H}), 2.85-2.63(\mathrm{~m}, 4 \mathrm{H})$, $2.55-2.45(\mathrm{~m}, 2 \mathrm{H}), 2.40(\mathrm{~s}, 3 \mathrm{H}), 2.37(\mathrm{~s}, 3 \mathrm{H}), 2.40-2.25(\mathrm{~m}, 3 \mathrm{H}), 2.02-1.95(\mathrm{~m}, 1 \mathrm{H})$, $1.37(\mathrm{~s}, 3 \mathrm{H}), 1.22(\mathrm{~s}, 3 \mathrm{H}), 1.14(\mathrm{~s}, 3 \mathrm{H}), 1.05(\mathrm{~s}, 3 \mathrm{H}), 1.00-0.90(\mathrm{~m}, 1 \mathrm{H})$.

${ }^{13}$ C NMR $\quad\left(126 \mathrm{MHz}, \mathrm{CD}_{2} \mathrm{Cl}_{2}\right) \delta 169.6,169.5,166.41,162.40,161.38,160.6,160.4,150.4,149.5$, $148.1,124.4,123.9,122.4,121.9,114.9,113.2,82.1,75.8,74.5,49.3,45.2,37.8,35.4$, $34.1,32.1,31.7,30.9,27.5,27.4,22.2,19.6,19.2$.

HRMS (ESI-TOF, m/z) calcd. for $\mathrm{C}_{33} \mathrm{H}_{41} \mathrm{O}_{7}[\mathrm{M}]^{+}$calc.: 549.2852; found: 549.2852.

IR $\quad\left(A T R\right.$, neat, $\left.\mathrm{cm}^{-1}\right) 3452($ br), $3199(\mathrm{br}), 2923(\mathrm{~s}), 2852(\mathrm{~m}), 1716(\mathrm{w}), 1594(\mathrm{~m}), 1448(\mathrm{~s})$, $1377(\mathrm{~m}), 1279(\mathrm{~m}), 1178(\mathrm{~s}), 1074(\mathrm{~m}), 908(\mathrm{w}), 734(\mathrm{w})$.

mp: $\quad 180-185^{\circ} \mathrm{C}$. 
$[\boldsymbol{\alpha}]_{\mathbf{D}}{ }^{23}+95.2\left(\mathrm{c}=0.29\right.$ in $\left.\mathrm{CHCl}_{3}\right)$.

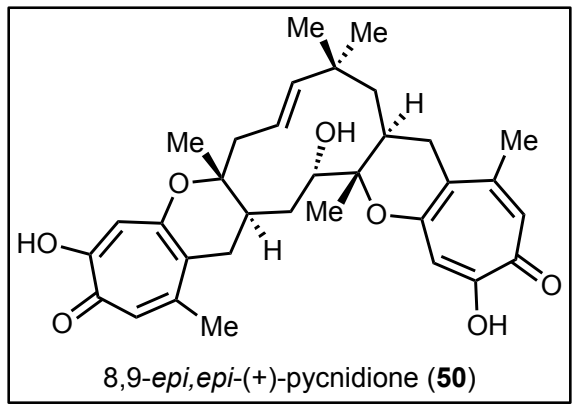

Synthesis of 8,9-Epi,epi-(+)-Pycnidione (50): Employing a procedure that is identical to that used for the conversion of $\mathbf{5 1}$ to 52, 49 (0.0039 g, 0.0067 mmol. 1.0 equiv.) was hydrolyzed in methanol $(0.20 \mathrm{~mL})$ and $1 \mathrm{M}$ sodium hydroxide $(0.20 \mathrm{~mL}, 30$ equiv. $)$ at $100{ }^{\circ} \mathrm{C}$ to give $\mathbf{5 0}$ as an orange solid $(0.0033 \mathrm{~g}, 0.0060 \mathrm{mmol}$,

$89 \%)$.

$\mathbf{R}_{\mathbf{f}}$

$0.2\left(\mathrm{SiO}_{2}, \mathrm{CH}_{2} \mathrm{Cl}_{2}: \mathrm{MeOH}=19: 1, \mathrm{UV}, \mathrm{KMnO}_{4}\right)$.

${ }^{1}$ H NMR $\quad\left(500 \mathrm{MHz}, \mathrm{CDCl}_{3}\right) \delta 7.18(\mathrm{~s}, 1 \mathrm{H}), 7.14(\mathrm{~s}, 1 \mathrm{H}), 6.99(\mathrm{~s}, 2 \mathrm{H}), 5.46(\mathrm{ddd}, J=14.3,8.9,5.1$ $\mathrm{Hz}, 1 \mathrm{H}), 5.36(\mathrm{~d}, J=15.8 \mathrm{~Hz}, 1 \mathrm{H}), 3.66(\mathrm{t}, J=4.5 \mathrm{~Hz}, 1 \mathrm{H}), 3.00(\mathrm{dd}, J=17.8,4.9 \mathrm{~Hz}, 1 \mathrm{H})$, $2.66(\mathrm{dd}, J=14.4,5.1 \mathrm{~Hz}, 1 \mathrm{H}), 2.60-2.49(\mathrm{~m}, 2 \mathrm{H}), 2.47-2.31(\mathrm{~m}, 3 \mathrm{H}), 2.43(\mathrm{~s}, 3 \mathrm{H}), 2.42$ $(\mathrm{s}, 3 \mathrm{H}), 2.10-2.02(\mathrm{~m}, 1 \mathrm{H}), 1.89(\mathrm{td}, J=11.8,4.2 \mathrm{~Hz}, 1 \mathrm{H}), 1.84-1.81(\mathrm{~m}, 1 \mathrm{H}), 1.51(\mathrm{~d}$, $J=14.7 \mathrm{~Hz}, 1 \mathrm{H}), 1.18(\mathrm{~s}, 3 \mathrm{H}), 1.15(\mathrm{~s}, 3 \mathrm{H}), 1.09(\mathrm{~s}, 3 \mathrm{H}), 1.05(\mathrm{~s}, 3 \mathrm{H}), 0.95-0.84(\mathrm{~m}, 1 \mathrm{H})$.

${ }^{13}$ C NMR $\quad\left(126 \mathrm{MHz}, \mathrm{CDCl}_{3}\right) \delta 172.7,169.6,167.5,163.3,162.7,160.9,150.5,145.9,143.8,129.4$, $124.8,122.6,122.1,120.8,117.2,113.5,88.7,80.1,78.1,45.5,40.2,37.8,36.2,33.1,32.6$, $30.5,29.8,29.6,27.4,26.8,23.9,19.2,16.4$.

HRMS (ESI-TOF, m/z) calcd. for $\mathrm{C}_{33} \mathrm{H}_{41} \mathrm{O}_{7}[\mathrm{M}]^{+}$calc.: 549.2852; found: 549.2847.

IR (ATR, neat, $\left.\mathrm{cm}^{-1}\right) 3397(\mathrm{br}), 3211(\mathrm{br}), 2925(\mathrm{~m}), 2855(\mathrm{w}), 1709(\mathrm{w}), 1588(\mathrm{~m}), 1443(\mathrm{~s})$, $1376(\mathrm{~m}), 1262$ (s), 1069 (m), $908(\mathrm{w}), 732(\mathrm{~m})$.

mp $\quad 220-225^{\circ} \mathrm{C}($ decomp.).

$[\boldsymbol{\alpha}]_{\mathbf{D}}{ }^{23}-14.8\left(\mathrm{c}=0.19\right.$ in $\left.\mathrm{CHCl}_{3}\right)$, 


\section{Computational Chemistry}

\section{General Computational Methods}

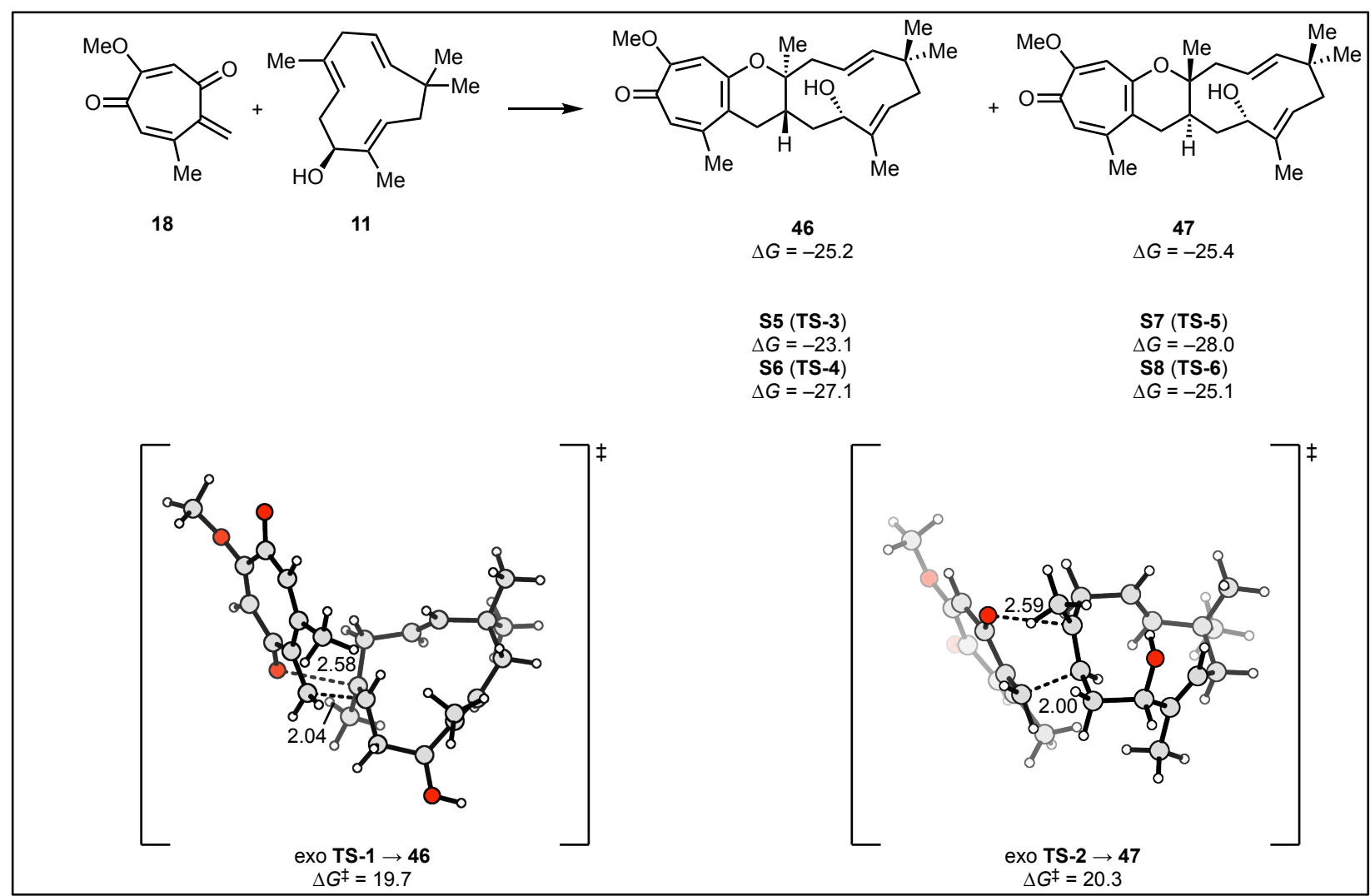

Figure S15. Computed cycloaddition and transition states to $\mathbf{4 6}$ and 47.

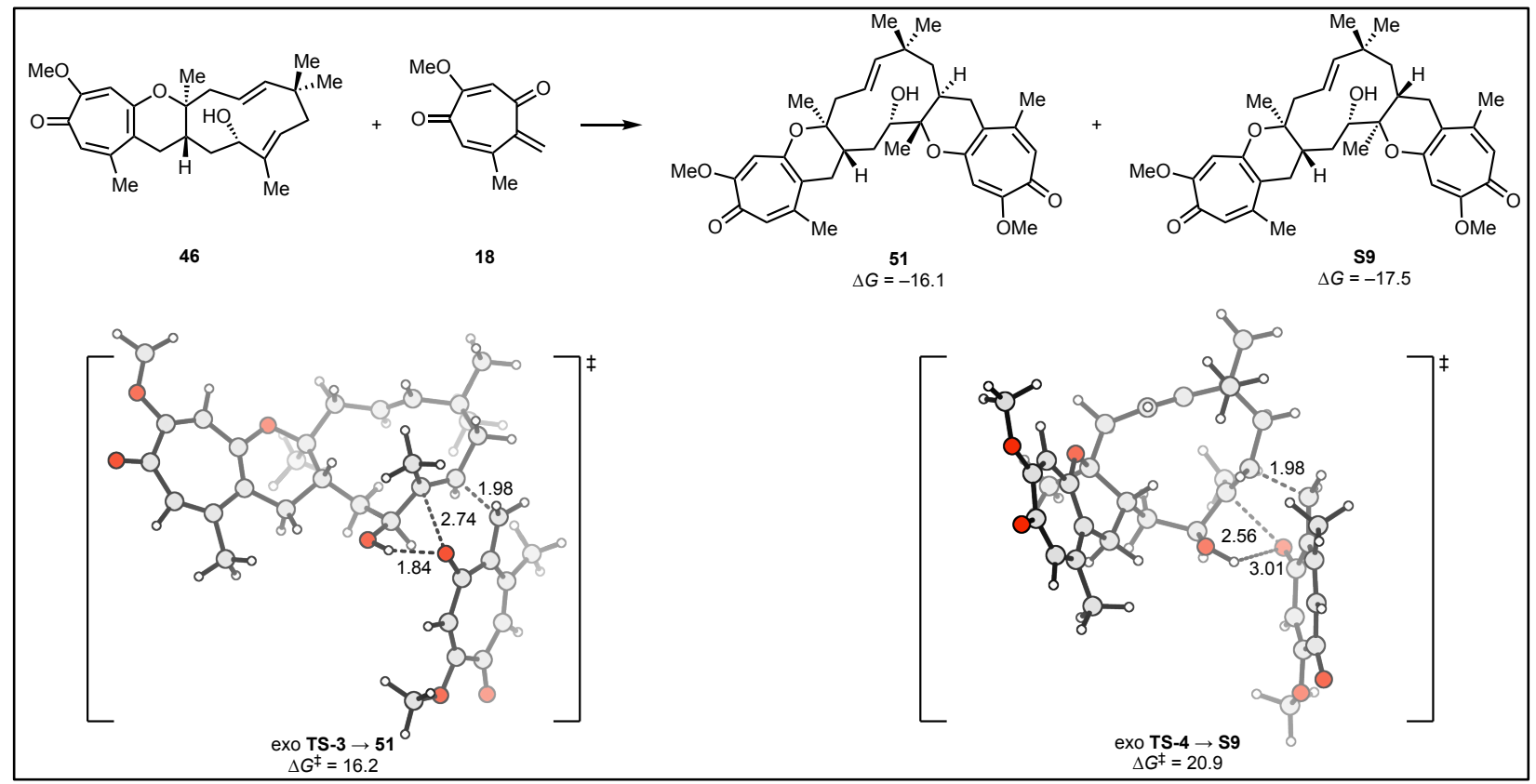

Figure S16. Computed cycloaddition and transition states to $\mathbf{5 1}$ and S9. 


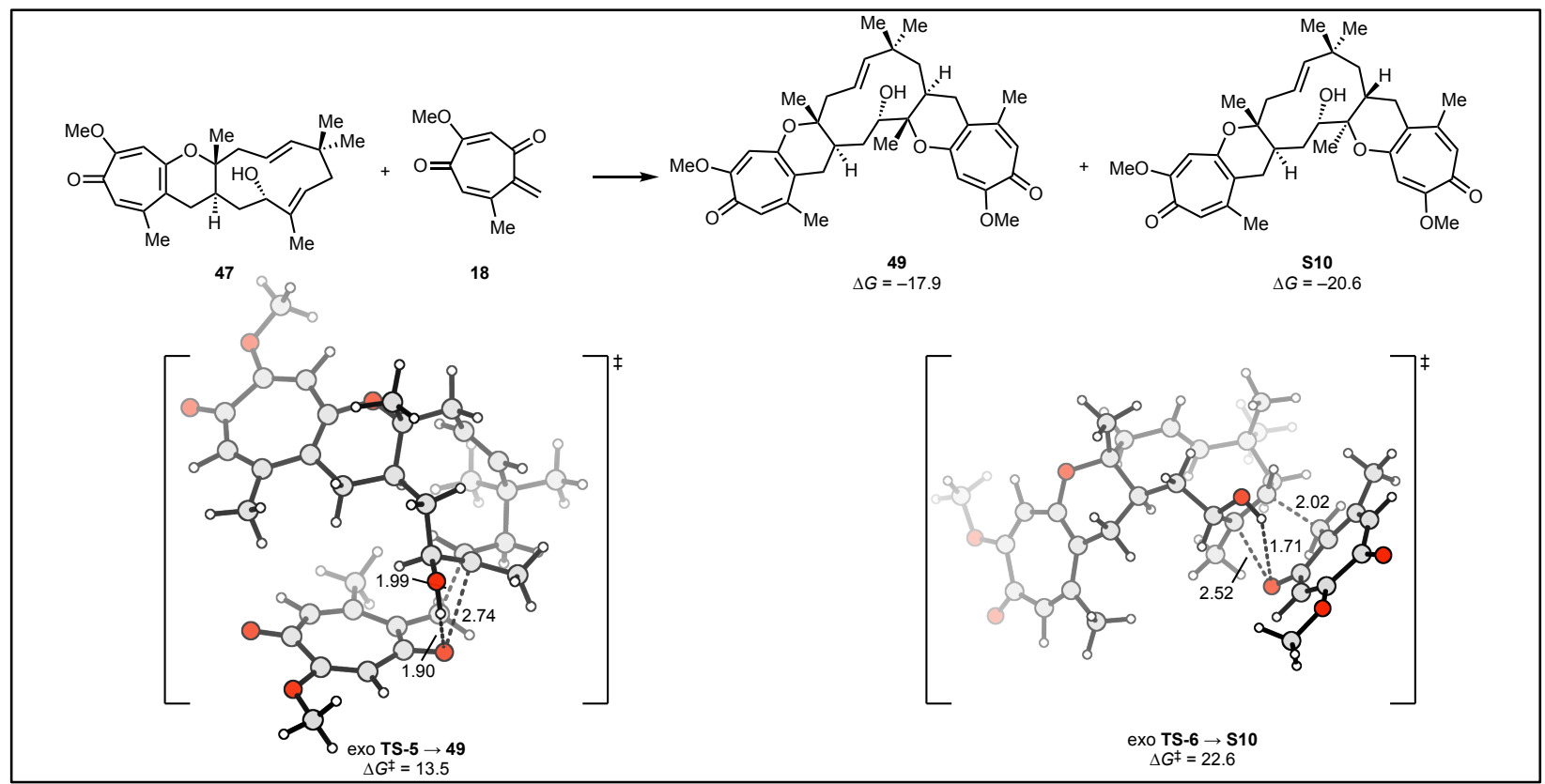

Figure S17. Computed cycloaddition and transition states to 49 and S10.

Conformational searches were completed with CREST conformer-rotamer ensemble sampling tool, version 2.7.1 and xtb version 6.2 RC2 (SAW190805). ${ }^{13}$ Output conformer geometries were optimized with quantum mechanics at the $\omega$ B97X-D/6-31G(d) level of theory as implemented in Gaussian 16 Rev. A.03 (sse4). ${ }^{14}$ Single point energetics were calculated at the $\omega$ B97X-D/def2-QZVPP-CPCM(solvent) level of theory with either an implicit water or mesitylene solvent. ${ }^{15}$ Thermochemistry was calculated using GoodVibes version 3.0.0 at $1 \mathrm{M}$ with quasiharmonic approximations to entropy and enthalpy. ${ }^{16}$ All reported structures are stationary points on the energy surface and characterized as transition states or minima by means of analytical frequency calculations.

Sample Gaussian input file for transition state optimization and frequency calculations:

$$
\begin{aligned}
& \% \text { nproc }=8 \\
& \% \text { mem }=16 \mathrm{~GB} \\
& \text { \#p wb97xd 6-31G(d) opt=(noeigen,calcfc,ts) freq } \\
& \text { Generated Title for TS-6a } \\
& 01 \\
& \begin{array}{llll}
\text { C } & -1.475932 & 0.033461 & 1.115819
\end{array} \\
& \begin{array}{llll}
\text { C } & -1.913117 & -1.434493 & 1.188844
\end{array} \\
& \begin{array}{llll}
\text { C } & -3.250619 & -1.581474 & 0.500999
\end{array} \\
& \begin{array}{llll}
\text { C } & -4.164596 & -0.644647 & 0.906009
\end{array} \\
& \begin{array}{llll}
\text { C } & -5.526379 & -0.424297 & 0.528065
\end{array} \\
& \begin{array}{llll}
\text { C } & -6.307271 & -1.052081 & -0.391178
\end{array} \\
& \begin{array}{llll}
\text { O } & -7.581290 & -0.704941 & -0.615500
\end{array} \\
& \begin{array}{llll}
\text { C } & -5.923808 & -2.168561 & -1.304496
\end{array} \\
& \begin{array}{llll}
\text { O } & -6.742895 & -2.562094 & -2.126376
\end{array} \\
& \begin{array}{llll}
\text { C } & -4.620461 & -2.809417 & -1.220754
\end{array}
\end{aligned}
$$




\begin{tabular}{|c|c|c|c|}
\hline C & -3.486390 & -2.598679 & -0.489005 \\
\hline $\mathrm{C}$ & -2.346410 & -3.563798 & -0.756515 \\
\hline $\mathrm{H}$ & -1.476813 & -3.050422 & -1.183993 \\
\hline $\mathrm{H}$ & -2.650039 & -4.339574 & -1.461587 \\
\hline $\mathrm{H}$ & -2.015111 & -4.056600 & 0.163964 \\
\hline $\mathrm{H}$ & -4.591903 & -3.635707 & -1.926393 \\
\hline $\mathrm{H}$ & -5.960111 & 0.394635 & 1.090469 \\
\hline $\mathrm{O}$ & -3.802939 & 0.225224 & 1.896336 \\
\hline $\mathrm{C}$ & -2.523365 & 0.905576 & 1.846198 \\
\hline $\mathrm{C}$ & -2.205584 & 1.159456 & 3.315056 \\
\hline $\mathrm{H}$ & -3.067959 & 1.633974 & 3.791579 \\
\hline $\mathrm{H}$ & -1.339420 & 1.815102 & 3.434576 \\
\hline $\mathrm{H}$ & -2.003494 & 0.219446 & 3.837388 \\
\hline $\mathrm{C}$ & -2.778568 & 2.221385 & 1.072276 \\
\hline $\mathrm{C}$ & -1.537713 & 3.015597 & 0.780514 \\
\hline $\mathrm{C}$ & -1.017888 & 3.158558 & -0.438269 \\
\hline $\mathrm{C}$ & 0.245250 & 3.891246 & -0.828216 \\
\hline $\mathrm{C}$ & 0.944453 & 4.536517 & 0.373610 \\
\hline $\mathrm{H}$ & 1.200138 & 3.806522 & 1.148148 \\
\hline $\mathrm{H}$ & 0.305330 & 5.299349 & 0.830695 \\
\hline $\mathrm{H}$ & 1.871648 & 5.025604 & 0.053788 \\
\hline $\mathrm{C}$ & -0.138657 & 4.995316 & -1.832410 \\
\hline $\mathrm{H}$ & -0.634974 & 4.574113 & -2.714353 \\
\hline $\mathrm{H}$ & 0.750628 & 5.538821 & -2.172585 \\
\hline $\mathrm{H}$ & -0.823346 & 5.714371 & -1.371120 \\
\hline $\mathrm{C}$ & 1.220078 & 2.921469 & -1.561587 \\
\hline $\mathrm{H}$ & 0.729410 & 2.533585 & -2.462192 \\
\hline $\mathrm{H}$ & 2.057334 & 3.536348 & -1.914406 \\
\hline $\mathrm{C}$ & 1.732755 & 1.773274 & -0.706431 \\
\hline $\mathrm{H}$ & 2.214515 & 2.064771 & 0.224917 \\
\hline $\mathrm{C}$ & 1.055904 & 0.563428 & -0.630711 \\
\hline $\mathrm{C}$ & 0.171879 & 0.073284 & -1.736462 \\
\hline $\mathrm{H}$ & -0.700111 & 0.719082 & -1.881681 \\
\hline $\mathrm{H}$ & 0.731099 & 0.049084 & -2.677730 \\
\hline $\mathrm{H}$ & -0.177329 & -0.943655 & -1.543135 \\
\hline $\mathrm{C}$ & 1.085304 & -0.218041 & 0.677313 \\
\hline $\mathrm{O}$ & 2.261824 & 0.011754 & 1.424584 \\
\hline $\mathrm{H}$ & 2.987387 & -0.482599 & 1.024525 \\
\hline $\mathrm{C}$ & -0.043845 & 0.248696 & 1.613554 \\
\hline $\mathrm{H}$ & 0.109213 & -0.276688 & 2.563525 \\
\hline $\mathrm{H}$ & 0.146449 & 1.308840 & 1.804016 \\
\hline $\mathrm{H}$ & 0.977805 & -1.286154 & 0.448954 \\
\hline $\mathrm{H}$ & -1.557734 & 2.715993 & -1.279323 \\
\hline $\mathrm{H}$ & -1.035823 & 3.462341 & 1.637780 \\
\hline $\mathrm{H}$ & -3.270714 & 1.952119 & 0.129199 \\
\hline $\mathrm{H}$ & -3.492973 & 2.818701 & 1.652831 \\
\hline $\mathrm{H}$ & -1.988327 & -1.739744 & 2.242931 \\
\hline $\mathrm{H}$ & -1.149274 & -2.065339 & 0.734256 \\
\hline $\mathrm{H}$ & -1.525786 & 0.319569 & 0.062201 \\
\hline $\mathrm{C}$ & -8.168325 & 0.326563 & 0.146188 \\
\hline $\mathrm{H}$ & -8.160607 & 0.087645 & 1.216951 \\
\hline $\mathrm{H}$ & -7.658360 & 1.284401 & -0.016967 \\
\hline $\mathrm{H}$ & -9.198040 & 0.399997 & -0.203391 \\
\hline $\mathrm{C}$ & 3.414765 & 1.303325 & -1.717346 \\
\hline $\mathrm{C}$ & 4.262322 & 0.413134 & -1.038004 \\
\hline $\mathrm{C}$ & 3.776026 & -0.948505 & -1.073671 \\
\hline $\mathrm{C}$ & 4.421823 & -2.125328 & -0.492222 \\
\hline $\mathrm{C}$ & 5.600988 & -2.290186 & 0.150753 \\
\hline $\mathrm{O}$ & 6.043832 & -3.480520 & 0.572238 \\
\hline
\end{tabular}




$\begin{array}{rrrr}\mathrm{C} & 6.602020 & -1.231688 & 0.533671 \\ \mathrm{O} & 7.663538 & -1.593682 & 1.017613 \\ \mathrm{C} & 6.314793 & 0.193301 & 0.415631 \\ \mathrm{C} & 5.359890 & 0.911876 & -0.231799 \\ \mathrm{C} & 5.481611 & 2.419198 & -0.112986 \\ \mathrm{H} & 6.270541 & 2.691326 & 0.590350 \\ \mathrm{H} & 4.546549 & 2.868149 & 0.240827 \\ \mathrm{H} & 5.725338 & 2.877247 & -1.078637 \\ \mathrm{H} & 7.088747 & 0.755457 & 0.931006 \\ \mathrm{H} & 3.808251 & -3.001212 & -0.672791 \\ \mathrm{O} & 2.644544 & -1.147732 & -1.579013 \\ \mathrm{H} & 3.735431 & 2.332065 & -1.828464 \\ \mathrm{H} & 2.870802 & 0.903336 & -2.566753 \\ \mathrm{C} & 5.271751 & -4.635766 & 0.325353 \\ \mathrm{H} & 5.842304 & -5.464552 & 0.744066 \\ \mathrm{H} & 5.125618 & -4.794462 & -0.749877 \\ \mathrm{H} & 4.294688 & -4.575556 & 0.820631\end{array}$

\section{Benchmark of Computational Methods}

In order to validate our computational methods, we performed a small benchmark of density functionals and implicit solvent models. All structures were optimized with $\omega$ B97X-D and a small, Pople-style basis set $6-31 \mathrm{G}(\mathrm{d})$. Calculations at this level of theory reproduced all experiments. However, it is unusual to report such low-level of theory calculations when higher accuracy methods are readily available. We report the quasiharmonic corrected $\Delta \Delta G^{\ddagger}$ at $298 \mathrm{~K}$ for TS-1 and TS-2 in Table S1 at various levels of theory. We chose to evaluate the popular functionals $\omega$ B97X-D and M06-2X on the basis that they perform best in reproducing experimental barriers for pericyclic reactions. ${ }^{17}$ Some studies have shown that adding empirical dispersion (D3) to M06-2X gave better results and therefore included these results as well. We employed the recommended mean field Alhrichs' def2 basis sets as well as smaller, old-fashioned splitvalence Pople style basis sets. For solvent models, we chose to evaluate the CPCM and SMD methods for implicit solvation. ${ }^{18}$ Using the various combinations of these functionals, basis sets, and implicit solvent methods, we found that the $\Delta \Delta G^{\ddagger}$ ranges from -1.9 to +2.6 . In the main text, we chose to report the energies at the $\omega B$ 97X-D/def2-QZVPP-CPCM-(mesitylene) and $\omega$ B97X-D/def2-QZVPP-CPCM- $\left(\mathrm{H}_{2} \mathrm{O}\right)$ levels of theory as this method (1) is the recommended functional and basis set for calculating pericyclic reactions and (2) performed quite well in reproducing experiment.

Table S1. Benchmark of energetics using density functional theory and implicit solvent methods.

*Using transition state theory at $298 \mathrm{~K}$, the experimental d.r. of $1.4: 1$ corresponds to a $\Delta \Delta G^{\ddagger}=+0.3$. 
Singlepoint Method

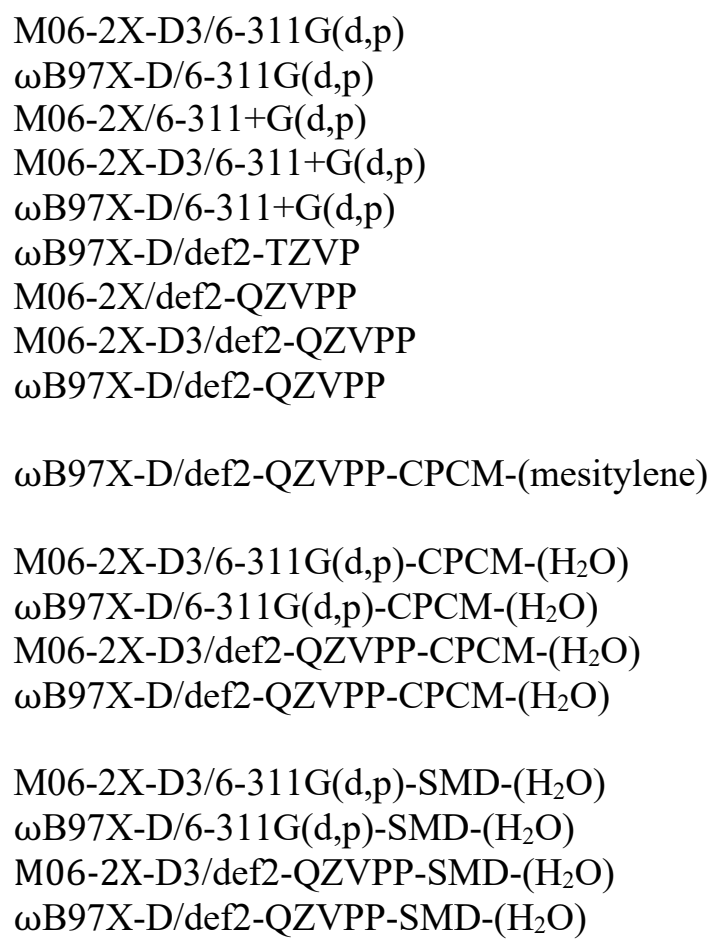

$$
\begin{aligned}
& -0.5 \\
& -0.8 \\
& -1.6 \\
& -1.4 \\
& -1.9 \\
& -1.5 \\
& -1.2 \\
& -1.0 \\
& -1.6 \\
& \\
& +0.6 \\
& \\
& +1.4 \\
& +1.2 \\
& +1.1 \\
& +0.5 \\
& \\
& +2.6 \\
& +2.5 \\
& +2.3 \\
& +2.0
\end{aligned}
$$




\section{Cartesian Coordinates of Calculated Structures}

\section{1}

$\begin{array}{lr}\mathrm{E} & -661.021842 \\ \mathrm{H} & -660.639718 \\ \mathrm{G} & -660.701177 \\ \text { Imag. Freq. } & 1.000\end{array}$

Cartesian coordinates

$\begin{array}{lrrr}\mathrm{C} & -1.536441 & 0.980078 & -0.862502 \\ \mathrm{C} & -1.263329 & 2.047597 & -0.108053 \\ \mathrm{C} & -2.178028 & 2.610715 & 0.946069 \\ \mathrm{H} & -3.155119 & 2.122573 & 0.967761 \\ \mathrm{H} & -2.345829 & 3.681299 & 0.770743 \\ \mathrm{H} & -1.727066 & 2.524201 & 1.943886 \\ \mathrm{C} & 0.093130 & 2.746574 & -0.224852 \\ \mathrm{C} & 1.197138 & 1.775082 & -0.581705 \\ \mathrm{C} & 1.665371 & 0.888176 & 0.293695 \\ \mathrm{C} & 2.549711 & -0.309048 & 0.031000 \\ \mathrm{C} & 2.987826 & -0.403564 & -1.433750 \\ \mathrm{H} & 3.611858 & -1.291342 & -1.586873 \\ \mathrm{H} & 2.132050 & -0.470039 & -2.113342 \\ \mathrm{H} & 3.576912 & 0.474167 & -1.721704 \\ \mathrm{C} & 3.797714 & -0.230697 & 0.926329 \\ \mathrm{H} & 4.413352 & 0.634388 & 0.657391 \\ \mathrm{H} & 4.410846 & -1.133927 & 0.821588 \\ \mathrm{H} & 3.520333 & -0.131798 & 1.982326 \\ \mathrm{C} & 1.739413 & -1.583649 & 0.439617 \\ \mathrm{C} & 0.410466 & -1.700250 & -0.253159 \\ \mathrm{C} & -0.804044 & -1.493099 & 0.269690 \\ \mathrm{C} & -1.086371 & -1.183797 & 1.715386 \\ \mathrm{H} & -2.039841 & -1.630456 & 2.013989 \\ \mathrm{H} & -0.307803 & -1.551622 & 2.387992 \\ \mathrm{H} & -1.171025 & -0.098731 & 1.858801 \\ \mathrm{C} & -2.011868 & -1.442539 & -0.651206 \\ \mathrm{O} & -3.049294 & -2.324296 & -0.248200 \\ \mathrm{H} & -2.672827 & -3.212351 & -0.218811 \\ \mathrm{H} & -1.675269 & -1.697465 & -1.669627 \\ \mathrm{H} & -2.633462 & -0.030670 & -0.696438 \\ \mathrm{H} & -3.193590 & 0.121910 & 0.231352 \\ \mathrm{H} & -3.360704 & -0.007912 & -1.517620 \\ \mathrm{H} & 0.463321 & -1.899341 & -1.324726 \\ & 1.605832 & -1.565598 & 1.527070 \\ \mathrm{H} & 2.361722 & -2.461282 & 0.214335 \\ \mathrm{H} & 1.302063 & 0.951108 & 1.323818 \\ \mathrm{H} & 0.314755 & 3.211316 & 0.745155 \\ \mathrm{H} & & 3.572323 & -0.946493 \\ \mathrm{H} & 0.692684 & -1.599247\end{array}$




\section{8}

$\begin{array}{lr}\text { E } & -612.476006 \\ H & -612.280353 \\ \text { G } & -612.333063 \\ \text { Imag. Freq. } & 1.000\end{array}$

Cartesian coordinates

\begin{tabular}{|c|c|c|}
\hline & & \\
\hline 1.527805 & 2.602769 & \\
\hline & & \\
\hline-1.373968 & & \\
\hline & & \\
\hline 971 & -0.96 & \\
\hline & & \\
\hline & -1 & \\
\hline 1.4 & -1. & \\
\hline & & \\
\hline 3.1 & & \\
\hline & -0 . & \\
\hline & & \\
\hline & -2. & \\
\hline & -1.6 & \\
\hline 2.1 & -3 & -0 . \\
\hline 3.1 & -2.3 & \\
\hline & -2.6 & -0.3 \\
\hline-0.8 & 2.47 & -0.23605 \\
\hline & 0.0348 & -0.42890 \\
\hline-3.370214 & -0.524009 & -1.30915 \\
\hline & -0.660066 & \\
\hline-4.498100 & 0.722712 & -0.7207 \\
\hline
\end{tabular}


$\begin{array}{lr}\text { E } & -1273.581388 \\ \mathrm{H} & -1272.997954 \\ \mathrm{G} & -1273.085354 \\ \text { Imag. Freq. } & 1.000\end{array}$

Cartesian coordinates

\begin{tabular}{|c|c|c|c|}
\hline & -0.705107 & -1.193208 & 0.597097 \\
\hline & 0.510012 & -1.993746 & 0.127017 \\
\hline & 1.778030 & -1.178426 & 0.004123 \\
\hline & 1.836130 & 0.030963 & 0.653404 \\
\hline & 2.893584 & 0.994524 & 0.702907 \\
\hline & 4.075704 & 1.100970 & 0.042310 \\
\hline & 4.769750 & 2.222253 & 0.327290 \\
\hline & 4.593126 & 0.186633 & -1.000888 \\
\hline $\mathrm{O}$ & 5.539279 & 0.538067 & -1.707913 \\
\hline U & 4.034286 & -1.142506 & -1.151614 \\
\hline & 2.862114 & -1.733292 & -0.766212 \\
\hline & 2.669286 & -3.158140 & -1.253479 \\
\hline & 3.592848 & -3.550154 & -1.683565 \\
\hline & 2.362704 & -3.825011 & -0.441144 \\
\hline & 1.892365 & -3.213108 & -2.024527 \\
\hline & 4.685581 & -1.737564 & -1.787271 \\
\hline $\mathrm{H}$ & 2.685745 & 018 & \\
\hline $\mathrm{O}$ & 0.816849 & 0.496479 & 1.423073 \\
\hline & -0.311325 & -0.317327 & 1.7 \\
\hline & 0.085815 & -1.130152 & 3.030842 \\
\hline & 0.361230 & -0.457950 & 3.848709 \\
\hline & -0.742522 & -1.762636 & 3.365964 \\
\hline & 0.945554 & -1.772537 & 2.818546 \\
\hline & -1.371218 & & \\
\hline C & -1.773956 & 1.554712 & 0.967073 \\
\hline C & -3.024996 & 1.735184 & 0.555151 \\
\hline $\mathrm{C}$ & -3.467755 & 2.278103 & -0.785382 \\
\hline C & -2.287843 & 2.721762 & -1.656490 \\
\hline F & -1.603504 & 1.893764 & -1.870157 \\
\hline & -1.712629 & 3.515589 & -1.168057 \\
\hline & -2.652733 & 3.113278 & -2.612532 \\
\hline & -4.428351 & 3.460677 & -0.588204 \\
\hline 1 & -4.852897 & 3.781963 & -1.546964 \\
\hline$\Pi$ & -5.258444 & 3.189128 & 0.074589 \\
\hline & -3.906180 & 4.313986 & -0.142408 \\
\hline & -4.246248 & 1.120341 & -1.502946 \\
\hline & -3.531093 & -0.197576 & -1.400305 \\
\hline & -3.776323 & -1.162698 & -0.507478 \\
\hline & -4.919006 & -1.159761 & 0.475254 \\
\hline & -5.676630 & -0.409374 & 0.242832 \\
\hline & -4.566876 & -0.959410 & 1.495738 \\
\hline & -5.413398 & -2.138630 & 0.498336 \\
\hline & -2.832051 & -2.342585 & -0.341784 \\
\hline & -2.030963 & -2.593340 & -1.480213 \\
\hline & -2.622097 & -2.636947 & -2.242137 \\
\hline & -1.890272 & -2.140758 & 0.864102 \\
\hline & -2.501053 & -1.784710 & 1.700591 \\
\hline & -1.505082 & -3.121197 & 1.168906 \\
\hline & -3.444806 & -3.233344 & -0.120894 \\
\hline & & & -2.03 \\
\hline
\end{tabular}




$\begin{array}{rrrr}\mathrm{H} & -4.388831 & 1.404742 & -2.553465 \\ \mathrm{H} & -5.245843 & 1.054413 & -1.060476 \\ \mathrm{H} & -3.839317 & 1.355209 & 1.176751 \\ \mathrm{H} & -0.943816 & 1.922260 & 0.368045 \\ \mathrm{H} & -2.243988 & 0.260340 & 2.617537 \\ \mathrm{H} & -0.917632 & 1.380471 & 2.937871 \\ \mathrm{H} & 0.245322 & -2.438675 & -0.834431 \\ \mathrm{H} & 0.675720 & -2.839550 & 0.811534 \\ \mathrm{H} & -0.986933 & -0.504446 & -0.206786 \\ \mathrm{C} & 6.196158 & 2.209763 & 0.341576 \\ \mathrm{H} & 6.579360 & 1.279529 & 0.773113 \\ \mathrm{H} & 6.602863 & 2.334496 & -0.660556 \\ \mathrm{H} & 6.477419 & 3.046875 & 0.984120\end{array}$


S5

$\begin{array}{lr}\text { E } & -1273.575602 \\ \mathrm{H} & -1272.991683 \\ \mathrm{G} & -1273.078806 \\ \text { Imag. Freq. } & 1.000\end{array}$

Cartesian coordinates

$\begin{array}{rrrr}\mathrm{C} & 0.350075 & 0.763175 & 0.444717 \\ \mathrm{C} & -0.898714 & 1.616545 & 0.687645 \\ \mathrm{C} & -2.208828 & 0.944264 & 0.349576 \\ \mathrm{C} & -2.220711 & -0.423006 & 0.272396 \\ \mathrm{C} & -3.286223 & -1.329755 & -0.057189 \\ \mathrm{C} & -4.586036 & -1.125019 & -0.387836 \\ \mathrm{O} & -5.424732 & -2.131452 & -0.678650 \\ \mathrm{C} & -5.310956 & 0.174086 & -0.480350 \\ \mathrm{O} & -6.491038 & 0.170165 & -0.812603 \\ \mathrm{C} & -4.647708 & 1.425699 & -0.164933 \\ \mathrm{C} & -3.372398 & 1.780990 & 0.171635 \\ \mathrm{C} & -3.156173 & 3.269112 & 0.382404 \\ \mathrm{H} & -2.672046 & 3.476364 & 1.342470 \\ \mathrm{H} & -2.513909 & 3.691603 & -0.398867 \\ \mathrm{H} & -4.105543 & 3.807040 & 0.359632 \\ \mathrm{H} & -5.367616 & 2.236062 & -0.245611 \\ \mathrm{H} & -2.932687 & -2.354038 & -0.030112 \\ \mathrm{O} & -1.112471 & -1.177993 & 0.497533 \\ \mathrm{C} & 0.136415 & -0.657261 & 0.998802 \\ \mathrm{C} & 0.069421 & -0.694958 & 2.525557 \\ \mathrm{H} & -0.159298 & -1.710456 & 2.861488 \\ \mathrm{H} & 1.018948 & -0.387575 & 2.974499 \\ \mathrm{H} & -0.715395 & -0.030262 & 2.899101 \\ \mathrm{C} & 1.109081 & -1.723824 & 0.436021 \\ \mathrm{C} & 2.581129 & -1.544463 & 0.671004 \\ \mathrm{C} & 3.480548 & -1.582754 & -0.310985 \\ \mathrm{C} & 4.964453 & -1.304869 & -0.234805 \\ \mathrm{C} & 5.729316 & -2.435646 & -0.941154 \\ \mathrm{H} & 5.381765 & -2.563771 & -1.972952 \\ \mathrm{H} & 5.588799 & -3.387242 & -0.417226 \\ \mathrm{H} & 6.803310 & -2.217617 & -0.971851 \\ \mathrm{C} & 5.466167 & -1.183579 & 1.207888 \\ \mathrm{H} & 6.541601 & -0.973706 & 1.219011 \\ \mathrm{H} & 5.298707 & -2.117036 & 1.756200 \\ \mathrm{H} & 4.963226 & -0.378859 & 1.753691 \\ \mathrm{C} & 5.227683 & 0.029224 & -1.016321 \\ \mathrm{C} & 4.413850 & 1.159130 & -0.461103 \\ \mathrm{C} & 3.209317 & 1.552135 & -0.888678 \\ \mathrm{C} & 2.575390 & 1.099696 & -2.179130 \\ \mathrm{H} & 2.059009 & 0.140200 & -2.059980 \\ \mathrm{H} & 3.317071 & 0.975675 & -2.973464 \\ \mathrm{H} & 1.828934 & 1.825755 & -2.512248 \\ \mathrm{C} & 2.368809 & 2.390880 & 0.065426 \\ \mathrm{O} & 1.456577 & 3.264660 & -0.579467 \\ \mathrm{H} & 1.966590 & 3.864118 & -1.137430 \\ \mathrm{C} & 1.573943 & 1.500086 & 1.035344 \\ \mathrm{H} & 2.298060 & 0.797805 & 1.453014 \\ \mathrm{H} & 1.233374 & 2.129625 & 1.866738 \\ \mathrm{H} & 3.062361 & 2.976492 & 0.689590 \\ \mathrm{H} & 4.771937 & 1.575082 & 0.482285 \\ & & & \end{array}$

S48 
H $\quad 4.991758 \quad-0.141534 \quad-2.073385$

$\begin{array}{llll}\mathrm{H} & 6.301704 & 0.249614 & -0.959549\end{array}$

$\mathrm{H} \quad 3.118682-1.761542-1.326343$

$\mathrm{H} \quad 2.904391 \quad-1.388016 \quad 1.699588$

$\mathrm{H} \quad 0.776195 \quad-2.680730 \quad 0.861278$

$\mathrm{H} \quad 0.920934 \quad-1.784488 \quad-0.642039$

$\mathrm{H} \quad-0.766754 \quad 2.530182 \quad 0.104333$

$\begin{array}{llll}\mathrm{H} & -0.913335 & 1.939100 & 1.739842\end{array}$

$\mathrm{H} \quad 0.468814 \quad 0.656302 \quad-0.638756$

$\begin{array}{llll}\text { C } & -4.956412 & -3.460000 & -0.652858\end{array}$

$\mathrm{H} \quad-4.147952 \quad-3.615095 \quad-1.378627$

$\mathrm{H} \quad-4.605020 \quad-3.742930 \quad 0.347636$

H $\quad-5.811926 \quad-4.078477 \quad-0.924885$ 
$\begin{array}{lr}\text { E } & -1273.582460 \\ \text { H } & -1272.998691 \\ \text { G } & -1273.085761 \\ \text { Imag. Freq. } & 1.000\end{array}$

Cartesian coordinates

$\begin{array}{rrrr}\mathrm{C} & -0.630941 & -1.191400 & 0.638443 \\ \mathrm{C} & 0.577546 & -2.016533 & 0.194837 \\ \mathrm{C} & 1.843863 & -1.211584 & -0.001441 \\ \mathrm{C} & 1.909621 & 0.036256 & 0.561752 \\ \mathrm{C} & 2.936566 & 1.039133 & 0.493691 \\ \mathrm{C} & 4.140684 & 1.069232 & -0.130771 \\ \mathrm{O} & 4.954865 & 2.134665 & -0.079898 \\ \mathrm{C} & 4.776485 & -0.010206 & -0.937878 \\ \mathrm{O} & 5.874709 & 0.196790 & -1.441756 \\ \mathrm{C} & 4.125373 & -1.295316 & -1.114144 \\ \mathrm{C} & 2.917295 & -1.820504 & -0.752983 \\ \mathrm{C} & 2.674558 & -3.245986 & -1.216343 \\ \mathrm{H} & 3.578296 & -3.671107 & -1.656457 \\ \mathrm{H} & 2.363183 & -3.893303 & -0.389918 \\ \mathrm{H} & 1.882555 & -3.288135 & -1.972751 \\ \mathrm{H} & 4.780688 & -1.946099 & -1.687317 \\ \mathrm{H} & 2.643233 & 1.918366 & 1.055747 \\ \mathrm{O} & 0.899800 & 0.551738 & 1.316042 \\ \mathrm{C} & -0.214713 & -0.238462 & 1.771405 \\ \mathrm{C} & 0.213870 & -0.968467 & 3.044167 \\ \mathrm{H} & 1.071311 & -1.620549 & 2.853758 \\ \mathrm{H} & 0.504793 & -0.244024 & 3.810526 \\ \mathrm{H} & -0.603740 & -1.580283 & 3.438635 \\ \mathrm{C} & -1.270485 & 0.843517 & 2.092588 \\ \mathrm{C} & -1.703018 & 1.572328 & 0.853698 \\ \mathrm{C} & -2.963540 & 1.730914 & 0.462218 \\ \mathrm{C} & -3.437504 & 2.182771 & -0.901187 \\ \mathrm{C} & -2.278319 & 2.566527 & -1.826870 \\ \mathrm{H} & -1.693407 & 3.391431 & -1.405853 \\ \mathrm{H} & -2.665337 & 2.893056 & -2.798475 \\ \mathrm{H} & -1.598130 & 1.725717 & -1.999896 \\ \mathrm{C} & -4.395085 & 3.375979 & -0.762267 \\ \mathrm{H} & -5.209381 & 3.149727 & -0.063859 \\ \mathrm{H} & -4.842022 & 3.631861 & -1.730478 \\ \mathrm{H} & -3.864394 & 4.257588 & -0.387058 \\ \mathrm{C} & -4.230207 & 0.979457 & -1.521600 \\ \mathrm{H} & -5.218004 & 0.941309 & -1.050231 \\ \mathrm{H} & -4.400572 & 1.195041 & -2.584317 \\ \mathrm{C} & -3.508979 & -0.328321 & -1.352640 \\ \mathrm{H} & -2.649420 & -0.489772 & -2.001878 \\ \mathrm{C} & -3.726759 & -1.232507 & -0.391581 \\ \mathrm{C} & -4.840765 & -1.164218 & 0.621338 \\ \mathrm{H} & -5.323980 & -2.142460 & 0.733388 \\ \mathrm{H} & -4.461717 & -0.883357 & 1.612765 \\ \mathrm{H} & -5.612680 & -0.440111 & 0.354746 \\ \mathrm{C} & -2.776270 & -2.399403 & -0.176363 \\ \mathrm{O} & -2.002080 & -2.719144 & -1.315948 \\ \mathrm{H} & -2.611805 & -2.819589 & -2.057559 \\ \mathrm{H} & -3.381710 & -3.275203 & 0.113563 \\ \mathrm{C} & -1.806253 & -2.123114 & 0.991597 \\ & & & \end{array}$

S50 


$\begin{array}{rrrr}\mathrm{H} & -2.397921 & -1.718536 & 1.819757 \\ \mathrm{H} & -1.410512 & -3.082606 & 1.345345 \\ \mathrm{H} & -3.763319 & 1.397399 & 1.127716 \\ \mathrm{H} & -0.888211 & 1.894113 & 0.208935 \\ \mathrm{H} & -2.131133 & 0.386377 & 2.594462 \\ \mathrm{H} & -0.802660 & 1.527670 & 2.812537 \\ \mathrm{H} & 0.293806 & -2.517825 & -0.732439 \\ \mathrm{H} & 0.757047 & -2.820553 & 0.924845 \\ \mathrm{H} & -0.932765 & -0.556814 & -0.201966 \\ \mathrm{C} & 4.558168 & 3.282629 & 0.634273 \\ \mathrm{H} & 5.373502 & 3.996976 & 0.518786 \\ \mathrm{H} & 3.635235 & 3.711881 & 0.223965 \\ \mathrm{H} & 4.413675 & 3.063090 & 1.699822\end{array}$




\section{7}

$\begin{array}{lr}\text { E } & -1273.580519 \\ \text { H } & -1272.996945 \\ \text { G } & -1273.084357 \\ \text { Imag. Freq. } & 1.000\end{array}$

Cartesian coordinates

$\begin{array}{rrrr}\mathrm{C} & 0.673228 & -1.119084 & 0.490843 \\ \mathrm{C} & -0.514621 & -1.934142 & -0.020639 \\ \mathrm{C} & -1.815012 & -1.162320 & -0.092010 \\ \mathrm{C} & -1.901638 & 0.019982 & 0.605261 \\ \mathrm{C} & -2.945337 & 0.999492 & 0.616870 \\ \mathrm{C} & -4.209014 & 1.009950 & 0.119460 \\ \mathrm{O} & -4.897639 & 2.137669 & 0.386236 \\ \mathrm{C} & -4.910151 & -0.118873 & -0.534876 \\ \mathrm{O} & -6.132088 & -0.071540 & -0.685954 \\ \mathrm{C} & -4.169206 & -1.261620 & -1.031064 \\ \mathrm{C} & -2.892097 & -1.724616 & -0.867043 \\ \mathrm{C} & -2.588718 & -3.029943 & -1.581094 \\ \mathrm{H} & -3.485356 & -3.433162 & -2.054636 \\ \mathrm{H} & -2.200520 & -3.788584 & -0.893361 \\ \mathrm{H} & -1.834548 & -2.887876 & -2.363996 \\ \mathrm{H} & -4.835883 & -1.889705 & -1.616410 \\ \mathrm{H} & -2.665324 & 1.903762 & 1.149429 \\ \mathrm{O} & -0.888255 & 0.489544 & 1.378297 \\ \mathrm{C} & 0.254860 & -0.314940 & 1.729470 \\ \mathrm{C} & -0.126299 & -1.195569 & 2.918219 \\ \mathrm{H} & 0.715762 & -1.828308 & 3.216293 \\ \mathrm{H} & -0.974774 & -1.842299 & 2.675704 \\ \mathrm{H} & -0.411325 & -0.571456 & 3.770083 \\ \mathrm{C} & 1.292620 & 0.749686 & 2.149274 \\ \mathrm{C} & 1.716549 & 1.601971 & 0.988072 \\ \mathrm{C} & 2.976022 & 1.792347 & 0.605941 \\ \mathrm{C} & 3.453260 & 2.409849 & -0.689968 \\ \mathrm{C} & 2.296417 & 2.908701 & -1.562094 \\ \mathrm{H} & 1.610587 & 2.099461 & -1.834866 \\ \mathrm{H} & 1.716190 & 3.679473 & -1.043435 \\ \mathrm{H} & 2.684827 & 3.348276 & -2.487470 \\ \mathrm{C} & 4.406811 & 3.578303 & -0.395064 \\ \mathrm{H} & 5.221855 & 3.265295 & 0.267871 \\ \mathrm{H} & 3.872756 & 4.401090 & 0.092141 \\ \mathrm{H} & 4.852766 & 3.960128 & -1.321294 \\ \mathrm{C} & 4.254381 & 1.299417 & -1.453934 \\ \mathrm{C} & 3.523395 & -0.012744 & -1.488737 \\ \mathrm{C} & 3.749888 & -1.056510 & -0.683208 \\ \mathrm{C} & 4.903672 & -1.154436 & 0.285778 \\ \mathrm{H} & 5.145231 & -2.204158 & 0.472869 \\ \mathrm{H} & 4.656209 & -0.703109 & 1.254897 \\ \mathrm{H} & 5.801217 & -0.654767 & -0.086940 \\ \mathrm{C} & 2.734002 & -2.179065 & -0.590800 \\ \mathrm{O} & 3.336569 & -3.463840 & -0.537346 \\ \mathrm{H} & 3.904249 & -3.548548 & -1.313575 \\ \mathrm{H} & 2.059496 & -2.122327 & -1.460909 \\ \mathrm{C} & 1.892195 & -2.034109 & 0.691818 \\ \mathrm{H} & 2.535499 & -1.673110 & 1.500989 \\ \mathrm{H} & 1.563619 & -3.037700 & 0.984375 \\ & 2.654278 & -0.057816 & -2.146755\end{array}$

S52 


$\begin{array}{rrrr}\mathrm{H} & 5.225110 & 1.179879 & -0.960664 \\ \mathrm{H} & 4.457709 & 1.663680 & -2.469488 \\ \mathrm{H} & 3.775849 & 1.381042 & 1.225868 \\ \mathrm{H} & 0.898594 & 2.003068 & 0.393146 \\ \mathrm{H} & 2.159547 & 0.259325 & 2.606287 \\ \mathrm{H} & 0.814009 & 1.354947 & 2.929835 \\ \mathrm{H} & -0.253932 & -2.317791 & -1.012347 \\ \mathrm{H} & -0.643959 & -2.825769 & 0.610472 \\ \mathrm{H} & 0.927608 & -0.373618 & -0.273051 \\ \mathrm{C} & -5.879425 & 2.628678 & -0.525649 \\ \mathrm{H} & -5.567092 & 2.478754 & -1.563652 \\ \mathrm{H} & -6.841928 & 2.145520 & -0.367155 \\ \mathrm{H} & -5.941696 & 3.699299 & -0.319079\end{array}$


$\begin{array}{lr}\text { E } & -1273.581902 \\ \mathrm{H} & -1272.998256 \\ \mathrm{G} & -1273.085693 \\ \text { Imag. Freq. } & 1.000\end{array}$

Cartesian coordinates

$\begin{array}{rrrr}\mathrm{C} & 0.613841 & -1.117674 & 0.555213 \\ \mathrm{C} & -0.558161 & -1.982736 & 0.092675 \\ \mathrm{C} & -1.860215 & -1.227014 & -0.069075 \\ \mathrm{C} & -1.972062 & -0.001254 & 0.536011 \\ \mathrm{C} & -3.043358 & 0.956406 & 0.510744 \\ \mathrm{C} & -4.245053 & 0.963121 & -0.119287 \\ \mathrm{O} & -5.104646 & 1.987961 & -0.025515 \\ \mathrm{C} & -4.828835 & -0.104142 & -0.981095 \\ \mathrm{O} & -5.920029 & 0.088788 & -1.504198 \\ \mathrm{C} & -4.140767 & -1.367393 & -1.176636 \\ \mathrm{C} & -2.916537 & -1.859812 & -0.825425 \\ \mathrm{C} & -2.631326 & -3.270231 & -1.311085 \\ \mathrm{H} & -1.855777 & -3.275944 & -2.085993 \\ \mathrm{H} & -3.527668 & -3.722294 & -1.739040 \\ \mathrm{H} & -2.281072 & -3.916720 & -0.499807 \\ \mathrm{H} & -4.775467 & -2.027720 & -1.761895 \\ \mathrm{H} & -2.789178 & 1.822419 & 1.110991 \\ \mathrm{O} & -0.979811 & 0.532244 & 1.299709 \\ \mathrm{C} & 0.168346 & -0.227891 & 1.723859 \\ \mathrm{C} & -0.218239 & -1.021675 & 2.970709 \\ \mathrm{H} & -1.059083 & -1.691385 & 2.767282 \\ \mathrm{H} & -0.517184 & -0.339069 & 3.771603 \\ \mathrm{H} & 0.625102 & -1.623719 & 3.323676 \\ \mathrm{H} & -0.691206 & -2.818602 & 0.795033 \\ \mathrm{H} & -0.276651 & -2.446783 & -0.858489 \\ \mathrm{H} & 0.870279 & -0.429399 & -0.259866 \\ \mathrm{C} & -4.764737 & 3.116858 & 0.747009 \\ \mathrm{H} & -5.611509 & 3.797767 & 0.660246 \\ \mathrm{H} & -4.615922 & 2.851656 & 1.801399 \\ \mathrm{H} & -3.860583 & 3.607038 & 0.364446 \\ \mathrm{C} & 1.186892 & 0.879315 & 2.075495 \\ \mathrm{C} & 1.616471 & 1.646943 & 0.858614 \\ \mathrm{C} & 2.878964 & 1.828276 & 0.482153 \\ \mathrm{C} & 3.368087 & 2.350721 & -0.850508 \\ \mathrm{C} & 4.301859 & 3.551627 & -0.632839 \\ \mathrm{H} & 5.110675 & 3.301932 & 0.063758 \\ \mathrm{H} & 3.749911 & 4.401949 & -0.218171 \\ \mathrm{H} & 4.756904 & 3.867434 & -1.579254 \\ \mathrm{C} & 2.218541 & 2.764642 & -1.775129 \\ \mathrm{H} & 1.620915 & 3.565274 & -1.325988 \\ \mathrm{H} & 1.547097 & 1.927677 & -1.994561 \\ \mathrm{H} & 2.615378 & 3.136922 & -2.726052 \\ \mathrm{C} & 4.195230 & 1.195971 & -1.514439 \\ \mathrm{C} & 3.480696 & -0.124655 & -1.461184 \\ \mathrm{C} & 3.706204 & -1.100983 & -0.574886 \\ \mathrm{C} & 4.844383 & -1.108107 & 0.417447 \\ \mathrm{H} & 5.745259 & -0.636321 & 0.017219 \\ \mathrm{H} & 5.088879 & -2.136320 & 0.697012 \\ \mathrm{H} & 4.579190 & -0.576879 & 1.340220 \\ \mathrm{C} & 2.703759 & -2.227863 & -0.414268\end{array}$




$\begin{array}{lrrr}\mathrm{O} & 3.322381 & -3.496277 & -0.255906 \\ \mathrm{H} & 3.902592 & -3.630913 & -1.015636 \\ \mathrm{C} & 1.840000 & -1.999980 & 0.841330 \\ \mathrm{H} & 1.518218 & -2.983622 & 1.201392 \\ \mathrm{H} & 2.466743 & -1.573217 & 1.631359 \\ \mathrm{H} & 2.042305 & -2.245663 & -1.295911 \\ \mathrm{H} & 2.624288 & -0.231772 & -2.128541 \\ \mathrm{H} & 5.158684 & 1.127048 & -0.997704 \\ \mathrm{H} & 4.411555 & 1.484761 & -2.551330 \\ \mathrm{H} & 3.674591 & 1.479515 & 1.144385 \\ \mathrm{H} & 0.802400 & 1.985971 & 0.221201 \\ \mathrm{H} & 0.689821 & 1.535185 & 2.801991 \\ \mathrm{H} & 2.053461 & 0.437251 & 2.579901\end{array}$




\section{S8}

$\begin{array}{lr}\text { E } & -1273.577746 \\ \text { H } & -1272.994259 \\ \text { G } & -1273.081484 \\ \text { Imag. Freq. } & 1.000\end{array}$

Cartesian coordinates

$\begin{array}{lrrr}\mathrm{C} & -0.507863 & 0.847703 & 0.993810 \\ \mathrm{C} & 0.644574 & 1.860822 & 1.024632 \\ \mathrm{C} & 1.870279 & 1.246346 & 0.390539 \\ \mathrm{C} & 2.158860 & 0.006576 & 0.896277 \\ \mathrm{C} & 3.215553 & -0.912898 & 0.608941 \\ \mathrm{C} & 4.236821 & -0.841951 & -0.286164 \\ \mathrm{O} & 5.150666 & -1.812925 & -0.419018 \\ \mathrm{C} & 4.519962 & 0.249706 & -1.263392 \\ \mathrm{O} & 5.464290 & 0.117912 & -2.033572 \\ \mathrm{C} & 3.715489 & 1.460940 & -1.310587 \\ \mathrm{C} & 2.612491 & 1.914815 & -0.644140 \\ \mathrm{C} & 2.125689 & 3.294150 & -1.046794 \\ \mathrm{H} & 1.115041 & 3.252979 & -1.470099 \\ \mathrm{H} & 2.782092 & 3.735867 & -1.798702 \\ \mathrm{H} & 2.092620 & 3.973125 & -0.187792 \\ \mathrm{H} & 4.129496 & 2.124898 & -2.065165 \\ \mathrm{H} & 3.142890 & -1.797924 & 1.230724 \\ \mathrm{O} & 1.369522 & -0.468154 & 1.904939 \\ \mathrm{C} & -0.076999 & -0.426041 & 1.766653 \\ \mathrm{C} & -0.556400 & -0.471169 & 3.212245 \\ \mathrm{H} & -0.067902 & -1.303726 & 3.726381 \\ \mathrm{H} & -1.638022 & -0.612159 & 3.275225 \\ \mathrm{H} & -0.295999 & 0.453388 & 3.736278 \\ \mathrm{C} & -0.453267 & -1.711145 & 0.989500 \\ \mathrm{C} & -1.908593 & -1.843381 & 0.639468 \\ \mathrm{C} & -2.379988 & -1.806831 & -0.606586 \\ \mathrm{C} & -3.825460 & -1.855949 & -1.055937 \\ \mathrm{C} & -4.784695 & -2.175516 & 0.096303 \\ \mathrm{H} & -4.740636 & -1.426819 & 0.893428 \\ \mathrm{H} & -4.549250 & -3.150039 & 0.537642 \\ \mathrm{H} & -5.816344 & -2.212028 & -0.270958 \\ \mathrm{C} & -3.970130 & -2.936925 & -2.140191 \\ \mathrm{H} & -3.283479 & -2.754718 & -2.975109 \\ \mathrm{H} & -4.990873 & -2.950235 & -2.539993 \\ \mathrm{H} & -3.749913 & -3.929394 & -1.732382 \\ \mathrm{C} & -4.190794 & -0.472948 & -1.693170 \\ \mathrm{H} & -3.619831 & -0.353260 & -2.621379 \\ \mathrm{H} & -5.252087 & -0.503705 & -1.974531 \\ \mathrm{C} & -3.910759 & 0.654878 & -0.748313 \\ \mathrm{H} & -4.532510 & 0.706264 & 0.144556 \\ \mathrm{C} & -2.870381 & 1.489926 & -0.810212 \\ \mathrm{C} & -1.913575 & 1.554345 & -1.979279 \\ \mathrm{H} & -1.429015 & 0.590795 & -2.173279 \\ \mathrm{H} & -2.428202 & 1.848813 & -2.901054 \\ \mathrm{H} & -1.118516 & 2.286072 & -1.802048 \\ \mathrm{C} & -2.545003 & 2.331697 & 0.418744 \\ \mathrm{O} & -3.699719 & 2.822572 & 1.077122 \\ \mathrm{H} & -4.264230 & 3.221088 & 0.403228 \\ \mathrm{C} & -1.830389 & 1.465292 & 1.467885 \\ \mathrm{H} & -1.668302 & 2.070731 & 2.368165 \\ & & & \end{array}$

S56 


$\begin{array}{rrrr}\mathrm{H} & -2.549572 & 0.682542 & 1.727672 \\ \mathrm{H} & -1.900575 & 3.176086 & 0.122273 \\ \mathrm{H} & -1.660894 & -1.694631 & -1.422614 \\ \mathrm{H} & -2.598507 & -1.956633 & 1.474744 \\ \mathrm{H} & 0.143597 & -1.719471 & 0.068430 \\ \mathrm{H} & -0.125225 & -2.566701 & 1.594414 \\ \mathrm{H} & 0.856477 & 2.131086 & 2.069610 \\ \mathrm{H} & 0.336428 & 2.776780 & 0.520374 \\ \mathrm{H} & -0.631451 & 0.542713 & -0.049466 \\ \mathrm{C} & 5.086490 & -2.949392 & 0.413097 \\ \mathrm{H} & 5.172433 & -2.675937 & 1.472166 \\ \mathrm{H} & 4.155481 & -3.508649 & 0.256056 \\ \mathrm{H} & 5.935247 & -3.570740 & 0.127451\end{array}$


$\begin{array}{lr}\text { E } & -1886.131618 \\ \mathrm{H} & -1885.346694 \\ \mathrm{G} & -1885.461563 \\ \text { Imag. Freq. } & 1.000\end{array}$

Cartesian coordinates

$\begin{array}{lrrr}\mathrm{C} & 1.644926 & 0.419063 & 0.261135 \\ \mathrm{C} & 2.252429 & -0.980544 & 0.364943 \\ \mathrm{C} & 3.753368 & -1.038330 & 0.186179 \\ \mathrm{C} & 4.380230 & 0.055301 & -0.361288 \\ \mathrm{C} & 5.776563 & 0.315994 & -0.536294 \\ \mathrm{C} & 6.882768 & -0.461648 & -0.410111 \\ \mathrm{O} & 8.036978 & 0.165297 & -0.714113 \\ \mathrm{C} & 6.911584 & -1.914217 & -0.121741 \\ \mathrm{O} & 7.943251 & -2.556025 & -0.325985 \\ \mathrm{C} & 5.751704 & -2.577765 & 0.440734 \\ \mathrm{C} & 4.431949 & -2.246670 & 0.582180 \\ \mathrm{C} & 3.565429 & -3.319679 & 1.216753 \\ \mathrm{H} & 4.142453 & -4.226491 & 1.405359 \\ \mathrm{H} & 2.717490 & -3.587279 & 0.577222 \\ \mathrm{H} & 3.154478 & -2.979949 & 2.174462 \\ \mathrm{H} & 6.031978 & -3.580048 & 0.754640 \\ \mathrm{H} & 5.977174 & 1.334816 & -0.854836 \\ \mathrm{O} & 3.699533 & 1.160471 & -0.765304 \\ \mathrm{C} & 2.268688 & 1.171937 & -0.924622 \\ \mathrm{C} & 1.939243 & 0.545451 & -2.279531 \\ \mathrm{H} & 2.423430 & 1.113288 & -3.079374 \\ \mathrm{H} & 0.860685 & 0.536355 & -2.464258 \\ \mathrm{H} & 2.301373 & -0.485615 & -2.332025 \\ \mathrm{C} & 1.988938 & 2.691509 & -0.901888 \\ \mathrm{C} & 0.561661 & 3.127697 & -1.070138 \\ \mathrm{C} & -0.132400 & 3.714515 & -0.097120 \\ \mathrm{C} & -1.577614 & 4.155113 & -0.095309 \\ \mathrm{C} & -2.224946 & 4.107569 & -1.482835 \\ \mathrm{H} & -3.265264 & 4.447670 & -1.425838 \\ \mathrm{H} & -2.227111 & 3.102023 & -1.915997 \\ \mathrm{H} & -1.693021 & 4.765638 & -2.177989 \\ \mathrm{C} & -1.624239 & 5.606449 & 0.418869 \\ \mathrm{H} & -2.656720 & 5.972374 & 0.458979 \\ \mathrm{H} & -1.051751 & 6.268906 & -0.238735 \\ \mathrm{H} & -1.199856 & 5.683102 & 1.426815 \\ \mathrm{C} & -2.348674 & 3.271472 & 0.922910 \\ \mathrm{H} & -1.795871 & 3.307594 & 1.869520 \\ \mathrm{H} & -3.308250 & 3.763559 & 1.130237 \\ \mathrm{C} & -2.624582 & 1.807408 & 0.505402 \\ \mathrm{H} & -2.137337 & 1.609461 & -0.453663 \\ \mathrm{C} & -4.124568 & 1.595086 & 0.292595 \\ \mathrm{C} & -4.523959 & 0.155380 & 0.048255 \\ \mathrm{C} & -3.636448 & -0.835423 & 0.392880 \\ \mathrm{C} & -3.755698 & -2.255682 & 0.273005 \\ \mathrm{C} & -4.664065 & -3.054401 & -0.346677 \\ \mathrm{O} & -4.377289 & -4.367872 & -0.283877 \\ \mathrm{C} & -5.829085 & -2.605615 & -1.145301 \\ \mathrm{O} & -6.389239 & -3.393282 & -1.907274 \\ \mathrm{C} & -6.355196 & -1.264344 & -0.975482 \\ & -5.832503 & -0.092253 & -0.504372\end{array}$

S58 


$\begin{array}{rrrr}\mathrm{C} & -6.746179 & 1.114421 & -0.622417 \\ \mathrm{H} & -7.760455 & 0.808699 & -0.885093 \\ \mathrm{H} & -6.794822 & 1.679310 & 0.314077 \\ \mathrm{H} & -6.397833 & 1.804696 & -1.399622 \\ \mathrm{H} & -7.348551 & -1.206582 & -1.413791 \\ \mathrm{H} & -2.937884 & -2.792015 & 0.745114 \\ \mathrm{O} & -2.435002 & -0.563935 & 0.971160 \\ \mathrm{C} & -2.100464 & 0.741312 & 1.482232 \\ \mathrm{C} & -2.714061 & 0.864947 & 2.877614 \\ \mathrm{H} & -2.498701 & 1.841997 & 3.319478 \\ \mathrm{H} & -3.799346 & 0.736915 & 2.833861 \\ \mathrm{H} & -2.298762 & 0.100684 & 3.535056 \\ \mathrm{C} & -0.552170 & 0.684617 & 1.551404 \\ \mathrm{O} & -0.124131 & -0.217209 & 2.559512 \\ \mathrm{H} & -0.478359 & -1.083273 & 2.312845 \\ \mathrm{C} & 0.105343 & 0.322545 & 0.216732 \\ \mathrm{H} & -0.199001 & -0.697369 & -0.045022 \\ \mathrm{H} & -0.292337 & 0.961109 & -0.568800 \\ \mathrm{H} & -0.195309 & 1.666339 & 1.879574 \\ \mathrm{H} & -4.672947 & 1.997506 & 1.156566 \\ \mathrm{H} & -4.426263 & 2.214813 & -0.558942 \\ \mathrm{H} & 0.377358 & 3.882880 & 0.855644 \\ \mathrm{H} & 0.096801 & 2.939923 & -2.037953 \\ \mathrm{H} & 2.378706 & 3.074343 & 0.048097 \\ \mathrm{H} & 2.608112 & 3.130155 & -1.694833 \\ \mathrm{H} & 1.769784 & -1.639136 & -0.372892 \\ \mathrm{H} & 1.967093 & -1.371754 & 1.345608 \\ \mathrm{H} & 1.941714 & 0.974067 & 1.160931 \\ \mathrm{C} & -5.412267 & -5.351632 & -0.293186 \\ \mathrm{H} & -6.266276 & -5.032795 & 0.312003 \\ \mathrm{H} & -5.747811 & -5.561065 & -1.307252 \\ \mathrm{H} & -4.958653 & -6.235560 & 0.159155 \\ \mathrm{C} & 9.253611 & -0.187070 & -0.056942 \\ \mathrm{H} & 9.083218 & -0.403501 & 1.002270 \\ \mathrm{H} & 9.721872 & -1.049115 & -0.528720 \\ \mathrm{H} & 9.886077 & 0.698682 & -0.146233 \\ & & & \end{array}$


$\begin{array}{lr}\text { E } & -1886.130277 \\ \mathrm{H} & -1885.344872 \\ \mathrm{G} & -1885.457336 \\ \text { Imag. Freq. } & 1.000\end{array}$

Cartesian coordinates

$\begin{array}{lrrr}\mathrm{C} & 1.782915 & -0.095768 & -1.268106 \\ \mathrm{C} & 2.531697 & -1.403962 & -1.034492 \\ \mathrm{C} & 3.859538 & -1.212223 & -0.326190 \\ \mathrm{C} & 4.373981 & 0.061888 & -0.233869 \\ \mathrm{C} & 5.503954 & 0.540523 & 0.500845 \\ \mathrm{C} & 6.508814 & -0.088065 & 1.164549 \\ \mathrm{O} & 7.416107 & 0.745700 & 1.709312 \\ \mathrm{C} & 6.759094 & -1.547517 & 1.205504 \\ \mathrm{O} & 7.853598 & -1.967490 & 1.583814 \\ \mathrm{C} & 5.716036 & -2.486504 & 0.841636 \\ \mathrm{C} & 4.507095 & -2.383823 & 0.209446 \\ \mathrm{C} & 3.767061 & -3.696774 & 0.025197 \\ \mathrm{H} & 2.835610 & -3.711383 & 0.603096 \\ \mathrm{H} & 4.376419 & -4.538243 & 0.358885 \\ \mathrm{H} & 3.497945 & -3.869292 & -1.021987 \\ \mathrm{H} & 6.035991 & -3.491521 & 1.104639 \\ \mathrm{H} & 5.571659 & 1.624632 & 0.506456 \\ \mathrm{O} & 3.774602 & 1.136084 & -0.820072 \\ \mathrm{C} & 2.763867 & 0.944867 & -1.826401 \\ \mathrm{C} & 3.442817 & 0.516232 & -3.128711 \\ \mathrm{H} & 4.066447 & -0.370102 & -2.985049 \\ \mathrm{H} & 4.084805 & 1.323099 & -3.494374 \\ \mathrm{H} & 2.696215 & 0.292826 & -3.897384 \\ \mathrm{C} & 2.154532 & 2.350928 & -2.008394 \\ \mathrm{C} & 1.228993 & 2.765247 & -0.901827 \\ \mathrm{C} & -0.052334 & 3.072199 & -1.098625 \\ \mathrm{C} & -1.126482 & 3.319948 & -0.065133 \\ \mathrm{C} & -1.811513 & 4.664250 & -0.362201 \\ \mathrm{H} & -2.204201 & 4.690723 & -1.385254 \\ \mathrm{H} & -2.649806 & 4.832560 & 0.323931 \\ \mathrm{H} & -1.105426 & 5.493771 & -0.250185 \\ \mathrm{C} & -0.574589 & 3.345232 & 1.363741 \\ \mathrm{H} & 0.156179 & 4.151905 & 1.485737 \\ \mathrm{H} & -1.387294 & 3.517501 & 2.079003 \\ \mathrm{H} & -0.080045 & 2.406830 & 1.637235 \\ \mathrm{C} & -2.181109 & 2.189223 & -0.234379 \\ \mathrm{H} & -3.006188 & 2.375918 & 0.466034 \\ \mathrm{H} & -2.618582 & 2.299049 & -1.230076 \\ \mathrm{C} & -1.617095 & 0.767351 & -0.017052 \\ \mathrm{C} & -2.100356 & 0.187019 & 1.324322 \\ \mathrm{C} & -3.568492 & -0.154261 & 1.225611 \\ \mathrm{C} & -3.844256 & -0.955568 & 0.146516 \\ \mathrm{C} & -5.052656 & -1.568614 & -0.281549 \\ \mathrm{C} & -6.333745 & -1.428856 & 0.162212 \\ \mathrm{O} & -7.232252 & -2.170389 & -0.508586 \\ \mathrm{C} & -6.812797 & -0.450523 & 1.169389 \\ \mathrm{O} & -8.014539 & -0.189941 & 1.235845 \\ \mathrm{C} & -5.895881 & 0.167965 & 2.112797 \\ \mathrm{C} & -4.538159 & 0.329495 & 2.164849 \\ \mathrm{C} & -4.010159 & 1.129261 & 3.340226 \\ & & & \end{array}$

S60 


$\begin{array}{rrrr}\mathrm{H} & -3.250402 & 0.568822 & 3.895345 \\ \mathrm{H} & -4.814425 & 1.383794 & 4.032958 \\ \mathrm{H} & -3.547020 & 2.066116 & 3.008068 \\ \mathrm{H} & -6.469164 & 0.658761 & 2.895565 \\ \mathrm{H} & -4.926762 & -2.257162 & -1.112143 \\ \mathrm{O} & -2.778275 & -1.337137 & -0.622045 \\ \mathrm{C} & -1.880618 & -0.299294 & -1.121834 \\ \mathrm{C} & -2.507175 & 0.192855 & -2.421853 \\ \mathrm{H} & -1.939253 & 1.011449 & -2.872264 \\ \mathrm{H} & -2.538184 & -0.637257 & -3.131634 \\ \mathrm{H} & -3.529014 & 0.543104 & -2.246846 \\ \mathrm{C} & -0.579537 & -1.099866 & -1.376546 \\ \mathrm{O} & -0.840557 & -2.257553 & -2.139407 \\ \mathrm{H} & -1.600211 & -2.688029 & -1.721929 \\ \mathrm{C} & 0.516525 & -0.298455 & -2.104645 \\ \mathrm{H} & 0.750976 & -0.847501 & -3.022462 \\ \mathrm{H} & 0.130113 & 0.676377 & -2.409502 \\ \mathrm{H} & -0.215679 & -1.394489 & -0.376962 \\ \mathrm{H} & -1.890517 & 0.902853 & 2.118386 \\ \mathrm{H} & -1.529527 & -0.723259 & 1.553385 \\ \mathrm{H} & -0.529928 & 0.867367 & 0.047096 \\ \mathrm{H} & -0.423645 & 3.078396 & -2.127996 \\ \mathrm{H} & 1.646968 & 2.747726 & 0.103446 \\ \mathrm{H} & 1.624450 & 2.383711 & -2.967102 \\ \mathrm{H} & 3.002634 & 3.042184 & -2.088557 \\ \mathrm{H} & 2.675388 & -1.919944 & -1.994793 \\ \mathrm{H} & 1.893888 & -2.068838 & -0.444107 \\ \mathrm{H} & 1.481833 & 0.293019 & -0.285921 \\ \mathrm{C} & 8.115521 & 0.392389 & 2.902250 \\ \mathrm{H} & 7.466065 & -0.146432 & 3.598653 \\ \mathrm{H} & 8.989509 & -0.217674 & 2.681207 \\ \mathrm{H} & 8.408430 & 1.346171 & 3.346267 \\ \mathrm{C} & -8.438259 & -2.619042 & 0.111018 \\ \mathrm{H} & -8.266981 & -2.902269 & 1.153481 \\ \mathrm{H} & -9.211415 & -1.854153 & 0.067795 \\ \mathrm{H} & -8.730715 & -3.501889 & -0.460819 \\ & & & \end{array}$




\section{S10}

$\begin{array}{lr}\text { E } & -1886.128911 \\ \mathrm{H} & -1885.343763 \\ \mathrm{G} & -1885.457117 \\ \text { Imag. Freq. } & 1.000\end{array}$

Cartesian coordinates

$\begin{array}{lrrr}\mathrm{C} & 1.641274 & 0.169615 & -0.284388 \\ \mathrm{C} & 2.310717 & -0.986315 & 0.457077 \\ \mathrm{C} & 3.808684 & -1.043936 & 0.226793 \\ \mathrm{C} & 4.430355 & 0.050290 & -0.329500 \\ \mathrm{C} & 5.804146 & 0.244094 & -0.677870 \\ \mathrm{C} & 6.871509 & -0.596004 & -0.720057 \\ \mathrm{O} & 8.003256 & -0.028974 & -1.183295 \\ \mathrm{C} & 6.867812 & -2.044689 & -0.413479 \\ \mathrm{O} & 7.816500 & -2.748164 & -0.763019 \\ \mathrm{C} & 5.785995 & -2.623261 & 0.360360 \\ \mathrm{C} & 4.499212 & -2.244453 & 0.626456 \\ \mathrm{C} & 3.681391 & -3.249341 & 1.417772 \\ \mathrm{H} & 4.316892 & -4.045946 & 1.808872 \\ \mathrm{H} & 3.170213 & -2.776353 & 2.262524 \\ \mathrm{H} & 2.910715 & -3.716838 & 0.793914 \\ \mathrm{H} & 6.078509 & -3.610713 & 0.709275 \\ \mathrm{H} & 6.017792 & 1.258455 & -1.001319 \\ \mathrm{O} & 3.758969 & 1.201883 & -0.612711 \\ \mathrm{C} & 2.459913 & 1.451378 & -0.034407 \\ \mathrm{C} & 2.692932 & 1.811033 & 1.436142 \\ \mathrm{H} & 3.279267 & 2.733554 & 1.494196 \\ \mathrm{H} & 1.761849 & 1.954439 & 1.986086 \\ \mathrm{H} & 3.255478 & 1.023980 & 1.944054 \\ \mathrm{C} & 1.975119 & 2.664692 & -0.852528 \\ \mathrm{C} & 0.756289 & 3.358932 & -0.318927 \\ \mathrm{C} & -0.390555 & 3.483012 & -0.984188 \\ \mathrm{C} & -1.671949 & 4.097021 & -0.437904 \\ \mathrm{C} & -2.693833 & 4.229750 & -1.576173 \\ \mathrm{H} & -2.896943 & 3.265258 & -2.055883 \\ \mathrm{H} & -2.321045 & 4.909195 & -2.350645 \\ \mathrm{H} & -3.643404 & 4.630933 & -1.203745 \\ \mathrm{C} & -1.403928 & 5.493728 & 0.147169 \\ \mathrm{H} & -2.335046 & 5.943253 & 0.511731 \\ \mathrm{H} & -0.977830 & 6.156952 & -0.612346 \\ \mathrm{H} & -0.702978 & 5.451709 & 0.987563 \\ \mathrm{C} & -2.222878 & 3.196713 & 0.696147 \\ \mathrm{H} & -1.472249 & 3.192640 & 1.494353 \\ \mathrm{H} & -3.106494 & 3.686827 & 1.127424 \\ \mathrm{C} & -2.593059 & 1.749798 & 0.294326 \\ \mathrm{H} & -2.183087 & 1.534240 & -0.698208 \\ \mathrm{C} & -4.110755 & 1.582060 & 0.196027 \\ \mathrm{C} & -4.564568 & 0.151202 & -0.008504 \\ \mathrm{C} & -3.680703 & -0.862873 & 0.273694 \\ \mathrm{C} & -3.846313 & -2.279627 & 0.169941 \\ \mathrm{C} & -4.820164 & -3.056054 & -0.373976 \\ \mathrm{O} & -4.563866 & -4.376410 & -0.326323 \\ \mathrm{C} & -6.031176 & -2.579391 & -1.082675 \\ \mathrm{O} & -6.670636 & -3.355265 & -1.792593 \\ \mathrm{C} & -6.505374 & -1.222949 & -0.882999 \\ \mathrm{C} & -5.917116 & -0.063464 & -0.460369\end{array}$

S62 


$\begin{array}{rrrr}\mathrm{C} & -6.804816 & 1.166938 & -0.517439 \\ \mathrm{H} & -6.496494 & 1.845035 & -1.321818 \\ \mathrm{H} & -6.769003 & 1.736141 & 0.417043 \\ \mathrm{H} & -7.843477 & 0.887656 & -0.702892 \\ \mathrm{H} & -7.527047 & -1.139724 & -1.245568 \\ \mathrm{H} & -3.009160 & -2.836057 & 0.580898 \\ \mathrm{O} & -2.432496 & -0.622502 & 0.761232 \\ \mathrm{C} & -2.038015 & 0.674484 & 1.243674 \\ \mathrm{C} & -2.574744 & 0.832335 & 2.668512 \\ \mathrm{H} & -2.333818 & 1.821210 & 3.070167 \\ \mathrm{H} & -3.660763 & 0.707288 & 2.687618 \\ \mathrm{H} & -2.125880 & 0.083565 & 3.321120 \\ \mathrm{C} & -0.485845 & 0.580772 & 1.252148 \\ \mathrm{O} & -0.058765 & -0.322730 & 2.259331 \\ \mathrm{H} & -0.409885 & -1.190083 & 2.012876 \\ \mathrm{H} & -0.114218 & 1.555862 & 1.569917 \\ \mathrm{C} & 0.108772 & 0.205804 & -0.111203 \\ \mathrm{H} & -0.265069 & -0.793137 & -0.369254 \\ \mathrm{H} & -0.294465 & 0.876408 & -0.870534 \\ \mathrm{H} & -4.580534 & 2.002459 & 1.096654 \\ \mathrm{H} & -4.462983 & 2.204734 & -0.632238 \\ \mathrm{H} & -0.447520 & 3.062899 & -1.990421 \\ \mathrm{H} & 0.840450 & 3.773221 & 0.686837 \\ \mathrm{H} & 2.817359 & 3.368152 & -0.877253 \\ \mathrm{H} & 1.803591 & 2.340228 & -1.885818 \\ \mathrm{H} & 1.853585 & -1.919173 & 0.105211 \\ \mathrm{H} & 2.076751 & -0.921063 & 1.524977 \\ \mathrm{H} & 1.785065 & -0.020811 & -1.357794 \\ \mathrm{C} & 9.280597 & -0.452920 & -0.709544 \\ \mathrm{H} & 9.256769 & -0.653643 & 0.366246 \\ \mathrm{H} & 9.620722 & -1.344345 & -1.233698 \\ \mathrm{H} & 9.944644 & 0.391887 & -0.903690 \\ \mathrm{C} & -5.621885 & -5.332109 & -0.248226 \\ \mathrm{H} & -6.417204 & -4.987792 & 0.419807 \\ \mathrm{H} & -6.041304 & -5.536901 & -1.231547 \\ \mathrm{H} & -5.157686 & -6.225894 & 0.173035 \\ & & & \end{array}$


$\begin{array}{lr}\text { E } & -1886.126559 \\ \mathrm{H} & -1885.341329 \\ \mathrm{G} & -1885.454754 \\ \text { Imag. Freq. } & 1.000\end{array}$

Cartesian coordinates

$\begin{array}{lrrr}\mathrm{C} & -1.651406 & 0.293133 & 0.311006 \\ \mathrm{C} & -2.282692 & -1.097054 & 0.392696 \\ \mathrm{C} & -3.784168 & -1.112769 & 0.204286 \\ \mathrm{C} & -4.379244 & -0.004175 & -0.348389 \\ \mathrm{C} & -5.758326 & 0.247662 & -0.635091 \\ \mathrm{C} & -6.900124 & -0.423399 & -0.330889 \\ \mathrm{O} & -8.027384 & 0.194425 & -0.734041 \\ \mathrm{C} & -7.007745 & -1.665868 & 0.468659 \\ \mathrm{O} & -8.100503 & -2.011580 & 0.918879 \\ \mathrm{C} & -5.845829 & -2.511451 & 0.665005 \\ \mathrm{C} & -4.496268 & -2.306670 & 0.585624 \\ \mathrm{C} & -3.639932 & -3.491734 & 0.994556 \\ \mathrm{H} & -2.860015 & -3.702852 & 0.255731 \\ \mathrm{H} & -3.137653 & -3.309533 & 1.951741 \\ \mathrm{H} & -4.249057 & -4.390219 & 1.108308 \\ \mathrm{H} & -6.163814 & -3.481037 & 1.040477 \\ \mathrm{H} & -5.913774 & 1.176139 & -1.176255 \\ \mathrm{O} & -3.666300 & 1.081285 & -0.755365 \\ \mathrm{C} & -2.234882 & 1.049908 & -0.894633 \\ \mathrm{C} & -1.906412 & 0.392782 & -2.236388 \\ \mathrm{H} & -0.826182 & 0.313203 & -2.392505 \\ \mathrm{H} & -2.329847 & -0.614427 & -2.289241 \\ \mathrm{H} & -2.333550 & 0.979535 & -3.054988 \\ \mathrm{C} & -1.915450 & 2.564222 & -0.901311 \\ \mathrm{C} & -0.476813 & 2.961649 & -1.071584 \\ \mathrm{C} & 0.179896 & 3.737849 & -0.208191 \\ \mathrm{C} & 1.645914 & 4.116946 & -0.201683 \\ \mathrm{C} & 1.750454 & 5.585076 & 0.248537 \\ \mathrm{H} & 1.206326 & 6.243073 & -0.437548 \\ \mathrm{H} & 1.331095 & 5.721408 & 1.252283 \\ \mathrm{H} & 2.796561 & 5.909905 & 0.274607 \\ \mathrm{C} & 2.301121 & 3.983973 & -1.581147 \\ \mathrm{H} & 2.282344 & 2.960117 & -1.968079 \\ \mathrm{H} & 1.791115 & 4.622466 & -2.310140 \\ \mathrm{H} & 3.349866 & 4.297971 & -1.531137 \\ \mathrm{C} & 2.372462 & 3.243522 & 0.864798 \\ \mathrm{H} & 3.346577 & 3.712529 & 1.056100 \\ \mathrm{H} & 1.819462 & 3.326908 & 1.805016 \\ \mathrm{C} & 2.608268 & 1.756661 & 0.493143 \\ \mathrm{C} & 4.103148 & 1.538707 & 0.237212 \\ \mathrm{C} & 4.513837 & 0.100179 & 0.016941 \\ \mathrm{C} & 3.636684 & -0.883804 & 0.404112 \\ \mathrm{C} & 3.705711 & -2.299895 & 0.211772 \\ \mathrm{C} & 4.691835 & -3.124266 & -0.227997 \\ \mathrm{O} & 4.359652 & -4.429466 & -0.227189 \\ \mathrm{C} & 6.084582 & -2.736866 & -0.553885 \\ \mathrm{O} & 6.949843 & -3.607258 & -0.657317 \\ \mathrm{C} & 6.430593 & -1.348791 & -0.790189 \\ \mathrm{C} & 5.808367 & -0.151725 & -0.563619 \\ & 6.615383 & 1.074061 & -0.952980\end{array}$

S64 


$\begin{array}{rrrr}\mathrm{H} & 6.132572 & 1.624316 & -1.769181 \\ \mathrm{H} & 7.614410 & 0.792527 & -1.289722 \\ \mathrm{H} & 6.726793 & 1.770141 & -0.114843 \\ \mathrm{H} & 7.442496 & -1.297254 & -1.183797 \\ \mathrm{H} & 2.789180 & -2.808724 & 0.496080 \\ \mathrm{O} & 2.448724 & -0.603779 & 0.998260 \\ \mathrm{C} & 2.109190 & 0.701190 & 1.501464 \\ \mathrm{C} & 2.732241 & 0.857160 & 2.886919 \\ \mathrm{H} & 3.824861 & 0.851384 & 2.835256 \\ \mathrm{H} & 2.421632 & 0.023737 & 3.523474 \\ \mathrm{H} & 2.402910 & 1.786430 & 3.356986 \\ \mathrm{C} & 0.560347 & 0.578844 & 1.626044 \\ \mathrm{O} & -0.000813 & 1.737744 & 2.207560 \\ \mathrm{H} & -0.127892 & 2.376409 & 1.494024 \\ \mathrm{C} & -0.113337 & 0.178410 & 0.300998 \\ \mathrm{H} & 0.298801 & 0.785360 & -0.504161 \\ \mathrm{H} & 0.186522 & -0.848158 & 0.074004 \\ \mathrm{H} & 0.383053 & -0.219594 & 2.356508 \\ \mathrm{H} & 4.370764 & 2.142081 & -0.636096 \\ \mathrm{H} & 4.678004 & 1.965559 & 1.072222 \\ \mathrm{H} & 2.092991 & 1.539028 & -0.447849 \\ \mathrm{H} & -0.382356 & 4.138525 & 0.642078 \\ \mathrm{H} & 0.038395 & 2.581263 & -1.953604 \\ \mathrm{H} & -2.308447 & 2.985723 & 0.031201 \\ \mathrm{H} & -2.514328 & 2.994112 & -1.714850 \\ \mathrm{H} & -2.022463 & -1.521213 & 1.368734 \\ \mathrm{H} & -1.805402 & -1.754097 & -0.349106 \\ \mathrm{H} & -1.959737 & 0.858429 & 1.198301 \\ \mathrm{C} & -9.183540 & -0.555430 & -1.105386 \\ \mathrm{H} & -8.909237 & -1.449404 & -1.674158 \\ \mathrm{H} & -9.761151 & -0.849323 & -0.230804 \\ \mathrm{H} & -9.757650 & 0.117266 & -1.745753 \\ \mathrm{C} & 4.925477 & -5.335226 & -1.174574 \\ \mathrm{H} & 5.034303 & -4.865713 & -2.156791 \\ \mathrm{H} & 5.895462 & -5.699455 & -0.841131 \\ \mathrm{H} & 4.204264 & -6.152373 & -1.242721\end{array}$




\section{TS-1}

$\begin{array}{lr}\text { E } & -1273.494156 \\ \mathrm{H} & -1272.914515 \\ \mathrm{G} & -1273.006134 \\ \text { Imag. Freq. } & -375.155\end{array}$

Cartesian coordinates

$\begin{array}{rrrr}\mathrm{C} & 0.994565 & -1.377872 & -0.201852 \\ \mathrm{C} & 0.654111 & -0.828606 & -1.425494 \\ \mathrm{C} & 0.987029 & -1.470248 & -2.733178 \\ \mathrm{H} & 1.976120 & -1.126725 & -3.069661 \\ \mathrm{H} & 0.252210 & -1.204954 & -3.495023 \\ \mathrm{H} & 1.023701 & -2.559768 & -2.655453 \\ \mathrm{C} & 0.096400 & 0.573490 & -1.428404 \\ \mathrm{C} & 1.259482 & 1.536138 & -1.355823 \\ \mathrm{C} & 1.729054 & 2.054419 & -0.223205 \\ \mathrm{C} & 3.039880 & 2.786688 & -0.034463 \\ \mathrm{C} & 3.665915 & 3.229448 & -1.362337 \\ \mathrm{H} & 3.935263 & 2.383762 & -2.003617 \\ \mathrm{H} & 2.975331 & 3.868047 & -1.923372 \\ \mathrm{H} & 4.579605 & 3.803112 & -1.172197 \\ \mathrm{C} & 2.819434 & 4.024194 & 0.848509 \\ \mathrm{H} & 2.186848 & 4.758557 & 0.338447 \\ \mathrm{H} & 3.775069 & 4.504630 & 1.089155 \\ \mathrm{H} & 2.330065 & 3.755025 & 1.791667 \\ \mathrm{C} & 4.004957 & 1.805230 & 0.712243 \\ \mathrm{C} & 3.989009 & 0.433440 & 0.100463 \\ \mathrm{C} & 3.702610 & -0.725720 & 0.700638 \\ \mathrm{C} & 3.491298 & -0.903955 & 2.182511 \\ \mathrm{H} & 3.848212 & -1.891687 & 2.490737 \\ \mathrm{H} & 4.018637 & -0.151703 & 2.773436 \\ \mathrm{H} & 2.426104 & -0.847320 & 2.447626 \\ \mathrm{C} & 3.467729 & -1.957246 & -0.151991 \\ \mathrm{O} & 4.294029 & -3.050688 & 0.220798 \\ \mathrm{H} & 5.209463 & -2.751451 & 0.159403 \\ \mathrm{H} & 3.646484 & -1.689067 & -1.205371 \\ \mathrm{C} & 2.024264 & -2.473545 & -0.022399 \\ \mathrm{H} & 1.926510 & -2.919009 & 0.974365 \\ \mathrm{H} & 1.884273 & -3.289076 & -0.739275 \\ \mathrm{H} & 4.122998 & 0.409784 & -0.981760 \\ \mathrm{H} & 3.704724 & 1.755624 & 1.764460 \\ \mathrm{H} & 5.014641 & 2.239449 & 0.689949 \\ \mathrm{H} & 1.186771 & 1.849730 & 0.703808 \\ \mathrm{H} & 1.790469 & 1.699944 & -2.292099 \\ \mathrm{H} & -0.550852 & 0.699839 & -0.549191 \\ \mathrm{H} & -0.510175 & 0.743433 & -2.321855 \\ \mathrm{H} & 0.962102 & -0.669115 & 0.625018 \\ \mathrm{C} & -2.377743 & -1.178978 & -0.894332 \\ \mathrm{C} & -3.608533 & -0.437923 & -1.163088 \\ \mathrm{C} & -2.180439 & -0.807093 & 1.663021 \\ \mathrm{C} & -4.399153 & 0.337733 & -0.392419 \\ \mathrm{C} & -3.165581 & 0.106847 & 1.879073 \\ \mathrm{C} & -4.150779 & 0.754678 & 1.023494 \\ \mathrm{H} & -3.916144 & -0.527404 & -2.201211 \\ \mathrm{O} & -3.254737 & 0.504260 & 2.886608 \\ \mathrm{O} & -5.813131 & 1.681886 & 1.483046 \\ & -540063 & 0.912271 & -1.034865\end{array}$

S66 


$\begin{array}{lrrr}\mathrm{C} & -1.352705 & -1.173841 & 2.880832 \\ \mathrm{H} & -1.675494 & -0.610579 & 3.758241 \\ \mathrm{H} & -1.441719 & -2.240488 & 3.115425 \\ \mathrm{H} & -0.288453 & -0.961563 & 2.721179 \\ \mathrm{C} & -1.820986 & -1.456737 & 0.420330 \\ \mathrm{C} & -0.721370 & -2.324493 & 0.353751 \\ \mathrm{H} & -0.283117 & -2.702535 & 1.270516 \\ \mathrm{H} & -0.694839 & -3.008030 & -0.486969 \\ \mathrm{O} & -1.762843 & -1.615790 & -1.889931 \\ \mathrm{C} & -6.698696 & 1.056747 & -0.380691 \\ \mathrm{H} & -6.880347 & 0.237085 & 0.321700 \\ \mathrm{H} & -6.758835 & 2.005649 & 0.150786 \\ \mathrm{H} & -7.443352 & 1.014336 & -1.178986\end{array}$




\section{TS-2}

$\begin{array}{lr}\text { E } & -1273.496099 \\ \mathrm{H} & -1272.916106 \\ \mathrm{G} & -1273.005494 \\ \text { Imag. Freq. } & -385.332\end{array}$

Cartesian coordinates

$\begin{array}{rrrr}\mathrm{C} & 2.398160 & -1.277642 & -0.263384 \\ \mathrm{C} & 3.599801 & -0.600274 & -0.750736 \\ \mathrm{C} & 1.929957 & 0.099427 & 1.882478 \\ \mathrm{C} & 4.369435 & 0.379126 & -0.227838 \\ \mathrm{C} & 2.958217 & 0.987645 & 1.870935 \\ \mathrm{C} & 4.152793 & 1.146736 & 1.048204 \\ \mathrm{H} & 3.887715 & -1.024231 & -1.706460 \\ \mathrm{H} & 2.964909 & 1.742741 & 2.652380 \\ \mathrm{O} & 4.996096 & 1.968386 & 1.376434 \\ \mathrm{O} & 5.476140 & 0.840789 & -0.828996 \\ \mathrm{C} & 0.903908 & 0.304229 & 2.981152 \\ \mathrm{H} & 0.906298 & -0.527756 & 3.694668 \\ \mathrm{H} & -0.109445 & 0.382536 & 2.570136 \\ \mathrm{H} & 1.107861 & 1.219258 & 3.540184 \\ \mathrm{C} & 1.710219 & -1.025052 & 0.992583 \\ \mathrm{C} & 0.658835 & -1.930024 & 1.218886 \\ \mathrm{H} & 0.165781 & -1.921909 & 2.185290 \\ \mathrm{H} & 0.764647 & -2.910604 & 0.766961 \\ \mathrm{O} & 1.899423 & -2.126291 & -1.032815 \\ \mathrm{C} & -1.023200 & -1.493995 & 0.226168 \\ \mathrm{C} & -0.602546 & -1.450101 & -1.095719 \\ \mathrm{C} & -0.690579 & -2.631302 & -2.013034 \\ \mathrm{H} & -0.133082 & -2.457896 & -2.934585 \\ \mathrm{H} & -1.732890 & -2.868340 & -2.258784 \\ \mathrm{H} & -0.263832 & -3.515799 & -1.529118 \\ \mathrm{C} & -0.134258 & -0.106224 & -1.595772 \\ \mathrm{C} & -1.302875 & 0.848209 & -1.716934 \\ \mathrm{C} & -1.659764 & 1.711900 & -0.766541 \\ \mathrm{C} & -2.931279 & 2.531828 & -0.696078 \\ \mathrm{C} & -2.587321 & 3.961652 & -0.250122 \\ \mathrm{H} & -3.499803 & 4.549812 & -0.098304 \\ \mathrm{H} & -2.027239 & 3.958645 & 0.692160 \\ \mathrm{H} & -1.975678 & 4.468914 & -1.003942 \\ \mathrm{C} & -3.663214 & 2.596980 & -2.042167 \\ \mathrm{H} & -4.544581 & 3.242046 & -1.958798 \\ \mathrm{H} & -4.005956 & 1.615688 & -2.385513 \\ \mathrm{H} & -3.010509 & 3.012849 & -2.817127 \\ \mathrm{C} & -3.858178 & 1.884529 & 0.392020 \\ \mathrm{C} & -3.992319 & 0.405165 & 0.181646 \\ \mathrm{C} & -3.662296 & -0.580174 & 1.020151 \\ \mathrm{C} & -3.325178 & -0.384046 & 2.476775 \\ \mathrm{H} & -3.703208 & 0.565515 & 2.861141 \\ \mathrm{H} & -3.770036 & -1.183560 & 3.081783 \\ \mathrm{H} & -2.035120 & -2.493076 & 0.753392 \\ \mathrm{C} & -2.243716 & -0.413470 & 2.665702 \\ \mathrm{O} & -3.488816 & -2.002983 & 0.497642 \\ \mathrm{H} & -3.820000 & -2.137861 & -0.867484 \\ & -254509 & -1.517492 & -1.349097 \\ \mathrm{H} & -3.167464 & -2.686242 & 1.022571 \\ & & -3.471640 & 0.277054\end{array}$

S68 


$\begin{array}{rrrr}\mathrm{H} & -1.895673 & -2.643388 & 1.830187 \\ \mathrm{H} & -4.287492 & 0.117329 & -0.824368 \\ \mathrm{H} & -4.833076 & 2.389184 & 0.342022 \\ \mathrm{H} & -3.436820 & 2.102709 & 1.379630 \\ \mathrm{H} & -1.021732 & 1.793354 & 0.118215 \\ \mathrm{H} & -1.918963 & 0.731794 & -2.607902 \\ \mathrm{H} & 0.401174 & -0.211660 & -2.543168 \\ \mathrm{H} & 0.576133 & 0.294591 & -0.853431 \\ \mathrm{H} & -1.131492 & -0.509935 & 0.676198 \\ \mathrm{C} & 5.887485 & 0.269498 & -2.050691 \\ \mathrm{H} & 5.134647 & 0.417103 & -2.835137 \\ \mathrm{H} & 6.805182 & 0.790365 & -2.324211 \\ \mathrm{H} & 6.091153 & -0.802877 & -1.941545\end{array}$




\section{TS-3}

$\begin{array}{lr}\text { E } & -1886.060833 \\ \mathrm{H} & -1885.278919 \\ \mathrm{G} & -1885.394622 \\ \text { Imag. Freq. } & -349.848\end{array}$

Cartesian coordinates

$\begin{array}{lrrr}\mathrm{C} & 1.388887 & 0.257413 & 0.415439 \\ \mathrm{C} & 1.963714 & -1.151073 & 0.599214 \\ \mathrm{C} & 3.445642 & -1.277902 & 0.336271 \\ \mathrm{C} & 4.202207 & -0.139096 & 0.392929 \\ \mathrm{C} & 5.606767 & 0.063012 & 0.166214 \\ \mathrm{C} & 6.604830 & -0.792206 & -0.170517 \\ \mathrm{O} & 7.871865 & -0.389112 & -0.349473 \\ \mathrm{C} & 6.511421 & -2.264012 & -0.388862 \\ \mathrm{O} & 7.521889 & -2.878449 & -0.710865 \\ \mathrm{C} & 5.256909 & -2.970972 & -0.205917 \\ \mathrm{C} & 3.975611 & -2.598037 & 0.084368 \\ \mathrm{C} & 2.973113 & -3.736798 & 0.144197 \\ \mathrm{H} & 2.399715 & -3.723437 & 1.076994 \\ \mathrm{H} & 2.250671 & -3.673472 & -0.677645 \\ \mathrm{H} & 3.476991 & -4.702162 & 0.071287 \\ \mathrm{H} & 5.425199 & -4.033318 & -0.362354 \\ \mathrm{H} & 5.865470 & 1.108013 & 0.292505 \\ \mathrm{O} & 3.666769 & 1.079038 & 0.679924 \\ \mathrm{C} & 2.304173 & 1.290443 & 1.096712 \\ \mathrm{C} & 2.270084 & 1.200486 & 2.622210 \\ \mathrm{H} & 2.994258 & 1.899701 & 3.049936 \\ \mathrm{H} & 1.278508 & 1.444875 & 3.016012 \\ \mathrm{H} & 2.532299 & 0.193523 & 2.960249 \\ \mathrm{C} & 2.095572 & 2.738843 & 0.595446 \\ \mathrm{C} & 0.733239 & 3.341714 & 0.755580 \\ \mathrm{C} & 0.027131 & 3.811983 & -0.271213 \\ \mathrm{C} & -1.370469 & 4.380780 & -0.260053 \\ \mathrm{C} & -1.352544 & 5.756784 & -0.949001 \\ \mathrm{H} & -0.944849 & 5.686219 & -1.963958 \\ \mathrm{H} & -0.734090 & 6.462816 & -0.385317 \\ \mathrm{H} & -2.365398 & 6.170239 & -1.019822 \\ \mathrm{C} & -1.931740 & 4.544463 & 1.157235 \\ \mathrm{H} & -2.952038 & 4.942316 & 1.117164 \\ \mathrm{H} & -1.320729 & 5.245542 & 1.735378 \\ \mathrm{H} & -1.962367 & 3.600046 & 1.710412 \\ \mathrm{C} & -2.286656 & 3.442104 & -1.101707 \\ \mathrm{C} & -2.313516 & 2.013972 & -0.593963 \\ \mathrm{C} & -1.434991 & 1.042239 & -1.050652 \\ \mathrm{C} & -0.781032 & 1.105329 & -2.391482 \\ \mathrm{H} & -0.054841 & 1.925400 & -2.429046 \\ \mathrm{H} & -1.523346 & 1.279807 & -3.178218 \\ \mathrm{H} & -0.265093 & 0.169910 & -2.608105 \\ \mathrm{C} & -1.130494 & -0.111075 & -0.124065 \\ \mathrm{O} & -0.720052 & -1.272652 & -0.786797 \\ \mathrm{H} & -1.429392 & -1.462836 & -1.433552 \\ \mathrm{C} & -0.068967 & 0.268044 & 0.929638 \\ \mathrm{H} & -0.348367 & 1.242386 & 1.334541 \\ \mathrm{H} & -0.156440 & -0.449836 & 1.753053 \\ \mathrm{H} & -2.070323 & -0.292948 & 0.437064 \\ \mathrm{H} & -2.578771 & 1.920173 & 0.459674\end{array}$




$\begin{array}{rrrr}\mathrm{H} & -1.957784 & 3.461987 & -2.147187 \\ \mathrm{H} & -3.293740 & 3.877603 & -1.088182 \\ \mathrm{H} & 0.479510 & 3.763543 & -1.264480 \\ \mathrm{H} & 0.330587 & 3.397819 & 1.766396 \\ \mathrm{H} & 2.840398 & 3.354106 & 1.117833 \\ \mathrm{H} & 2.372337 & 2.747384 & -0.464685 \\ \mathrm{H} & 1.392772 & -1.807264 & -0.058111 \\ \mathrm{H} & 1.743067 & -1.494147 & 1.621593 \\ \mathrm{H} & 1.400051 & 0.489944 & -0.657408 \\ \mathrm{C} & -4.071158 & 1.317712 & -1.273302 \\ \mathrm{C} & -4.479684 & 0.129183 & -0.639520 \\ \mathrm{C} & -3.878542 & -1.051479 & -1.218757 \\ \mathrm{C} & -4.122911 & -2.436344 & -0.835308 \\ \mathrm{C} & -4.913719 & -2.997045 & 0.108135 \\ \mathrm{O} & -5.004498 & -4.318976 & 0.290892 \\ \mathrm{C} & -5.796695 & -2.303735 & 1.111217 \\ \mathrm{O} & -6.474582 & -2.996941 & 1.854774 \\ \mathrm{C} & -5.840444 & -0.853271 & 1.251420 \\ \mathrm{C} & -5.312316 & 0.182276 & 0.547881 \\ \mathrm{C} & -5.668026 & 1.565435 & 1.063063 \\ \mathrm{H} & -6.214121 & 1.500445 & 2.005673 \\ \mathrm{H} & -6.302086 & 2.104004 & 0.349096 \\ \mathrm{H} & -4.775758 & 2.178503 & 1.236583 \\ \mathrm{H} & -6.469177 & -0.600596 & 2.100798 \\ \mathrm{H} & -3.541452 & -3.097641 & -1.468107 \\ \mathrm{O} & -3.012136 & -0.883382 & -2.119709 \\ \mathrm{H} & -3.768192 & 1.235619 & -2.311184 \\ \mathrm{H} & -4.591409 & 2.235271 & -1.026011 \\ \mathrm{C} & 8.200633 & 0.971702 & -0.190584 \\ \mathrm{H} & 7.655482 & 1.602504 & -0.904247 \\ \mathrm{H} & 7.995539 & 1.317908 & 0.830473 \\ \mathrm{H} & 9.270358 & 1.040660 & -0.388763 \\ \mathrm{C} & -4.252162 & -5.188987 & -0.527797 \\ \mathrm{H} & -4.496452 & -6.195031 & -0.187389 \\ \mathrm{H} & -4.528488 & -5.080501 & -1.583540 \\ \mathrm{H} & -3.175774 & -5.012604 & -0.412175\end{array}$




\section{TS-4}

$\begin{array}{lr}\text { E } & -1886.052965 \\ \mathrm{H} & -1885.271301 \\ \mathrm{G} & -1885.386542 \\ \text { Imag. Freq. } & -403.741\end{array}$

Cartesian coordinates

$\begin{array}{rrrr}\mathrm{C} & -1.165627 & 0.308493 & -1.334155 \\ \mathrm{C} & -1.494858 & -1.183563 & -1.308149 \\ \mathrm{C} & -2.831737 & -1.506325 & -0.673499 \\ \mathrm{C} & -3.732286 & -0.487754 & -0.499454 \\ \mathrm{C} & -5.024513 & -0.477443 & 0.127556 \\ \mathrm{C} & -5.752389 & -1.452472 & 0.728062 \\ \mathrm{O} & -6.954722 & -1.222961 & 1.276389 \\ \mathrm{C} & -5.398808 & -2.892641 & 0.877736 \\ \mathrm{O} & -6.180809 & -3.635690 & 1.459100 \\ \mathrm{C} & -4.161118 & -3.421588 & 0.332942 \\ \mathrm{C} & -3.078712 & -2.881347 & -0.300128 \\ \mathrm{C} & -1.981537 & -3.870261 & -0.651483 \\ \mathrm{H} & -2.303742 & -4.894422 & -0.456117 \\ \mathrm{H} & -1.695092 & -3.801159 & -1.706058 \\ \mathrm{H} & -1.077352 & -3.686994 & -0.059853 \\ \mathrm{H} & -4.127128 & -4.492812 & 0.513748 \\ \mathrm{H} & -5.454115 & 0.517241 & 0.094063 \\ \mathrm{O} & -3.482179 & 0.786222 & -0.921463 \\ \mathrm{C} & -2.399775 & 1.084322 & -1.819619 \\ \mathrm{C} & -2.830087 & 0.717745 & -3.239855 \\ \mathrm{H} & -3.124203 & -0.333420 & -3.305597 \\ \mathrm{H} & -3.687797 & 1.327274 & -3.539533 \\ \mathrm{H} & -2.015436 & 0.893697 & -3.950123 \\ \mathrm{C} & -2.259758 & 2.613461 & -1.681253 \\ \mathrm{C} & -1.703740 & 3.003679 & -0.345257 \\ \mathrm{C} & -0.650823 & 3.796863 & -0.170122 \\ \mathrm{C} & 0.072754 & 4.083157 & 1.123048 \\ \mathrm{C} & -0.572406 & 3.386237 & 2.326267 \\ \mathrm{H} & -1.601192 & 3.731577 & 2.472641 \\ \mathrm{H} & -0.010918 & 3.614156 & 3.239152 \\ \mathrm{H} & -0.600989 & 2.297681 & 2.210287 \\ \mathrm{C} & 0.088319 & 5.601621 & 1.371478 \\ \mathrm{H} & 0.515976 & 6.138399 & 0.516709 \\ \mathrm{H} & 0.686169 & 5.846472 & 2.257394 \\ \mathrm{H} & -0.928052 & 5.976249 & 1.530802 \\ \mathrm{C} & 1.549275 & 3.613583 & 0.966222 \\ \mathrm{H} & 2.056406 & 4.275367 & 0.255215 \\ \mathrm{H} & 2.042836 & 3.777082 & 1.932484 \\ \mathrm{C} & 1.704950 & 2.165924 & 0.521706 \\ \mathrm{H} & 1.123181 & 1.434285 & 1.080011 \\ \mathrm{C} & 1.848628 & 1.841771 & -0.823649 \\ \mathrm{C} & 2.292189 & 2.824486 & -1.862330 \\ \mathrm{H} & 2.588230 & 2.315086 & -2.781918 \\ \mathrm{C} & 0.109638 & 0.573656 & -2.154321 \\ \mathrm{H} & 1.491201 & 3.534171 & -2.099490 \\ \mathrm{C} & 3.156343 & 3.393721 & -1.510347 \\ \mathrm{O} & 1.427405 & 0.474972 & -1.337793 \\ & 1.243828 & -0.471317 & -0.308578 \\ & 2.108107 & -0.719540 & 0.044342 \\ & 0.216507 & 0.139922 & -2.021968 \\ & & & \end{array}$

S72 


$\begin{array}{rrrr}\mathrm{H} & 0.071358 & 1.559347 & -2.629914 \\ \mathrm{H} & 0.162117 & -0.154060 & -2.972054 \\ \mathrm{H} & -0.209009 & 4.272582 & -1.048476 \\ \mathrm{H} & -2.184329 & 2.532502 & 0.509769 \\ \mathrm{H} & -1.632055 & 3.005251 & -2.489749 \\ \mathrm{H} & -3.264903 & 3.029722 & -1.825009 \\ \mathrm{H} & -0.687796 & -1.681282 & -0.768571 \\ \mathrm{H} & -1.460544 & -1.581086 & -2.334063 \\ \mathrm{H} & -0.987705 & 0.628217 & -0.302132 \\ \mathrm{C} & -7.497127 & 0.077319 & 1.250767 \\ \mathrm{H} & -8.458860 & 0.004581 & 1.758734 \\ \mathrm{H} & -6.855257 & 0.790495 & 1.783450 \\ \mathrm{H} & -7.653204 & 0.427082 & 0.222303 \\ \mathrm{C} & 3.444266 & 1.703785 & 1.336984 \\ \mathrm{C} & 3.884176 & 0.376110 & 1.146019 \\ \mathrm{C} & 4.403818 & 0.160858 & -0.187798 \\ \mathrm{C} & 4.987861 & -1.072730 & -0.711616 \\ \mathrm{C} & 5.200506 & -2.287121 & -0.155461 \\ \mathrm{O} & 5.798211 & -3.294178 & -0.805453 \\ \mathrm{C} & 4.803222 & -2.760072 & 1.217608 \\ \mathrm{O} & 5.127199 & -3.889869 & 1.551976 \\ \mathrm{C} & 4.005419 & -1.946925 & 2.128334 \\ \mathrm{C} & 3.617597 & -0.642133 & 2.141086 \\ \mathrm{C} & 2.813568 & -0.210425 & 3.353124 \\ \mathrm{H} & 1.860753 & 0.244662 & 3.059504 \\ \mathrm{H} & 2.590999 & -1.062992 & 3.996930 \\ \mathrm{H} & 3.361898 & 0.525060 & 3.952855 \\ \mathrm{H} & 3.728912 & -2.548916 & 2.989514 \\ \mathrm{H} & 5.308515 & -0.913776 & -1.735367 \\ \mathrm{O} & 4.288337 & 1.101798 & -1.009497 \\ \mathrm{H} & 3.144882 & 2.001609 & 2.335567 \\ \mathrm{H} & 3.984172 & 2.463714 & 0.781200 \\ \mathrm{C} & 6.267615 & -3.089819 & -2.119906 \\ \mathrm{H} & 7.014037 & -2.287016 & -2.156411 \\ \mathrm{H} & 5.444698 & -2.852218 & -2.805582 \\ \mathrm{H} & 6.728035 & -4.032094 & -2.416681\end{array}$




\section{TS-5}

$\begin{array}{cr}\text { E } & -1886.066873 \\ \text { H } & -1885.284968 \\ \text { G } & -1885.399232 \\ \text { Imag. Freq. } & -358.324\end{array}$

Cartesian coordinates

$\begin{array}{rrrr}\mathrm{C} & -0.579505 & 0.736347 & 1.448595 \\ \mathrm{C} & -0.775362 & -0.778059 & 1.445821 \\ \mathrm{C} & -2.109143 & -1.206461 & 0.866034 \\ \mathrm{C} & -3.096998 & -0.264978 & 0.736928 \\ \mathrm{C} & -4.413837 & -0.365594 & 0.170314 \\ \mathrm{C} & -5.041679 & -1.370460 & -0.490294 \\ \mathrm{O} & -6.285277 & -1.250408 & -0.978538 \\ \mathrm{C} & -4.511943 & -2.731663 & -0.790774 \\ \mathrm{O} & -5.167135 & -3.475152 & -1.510930 \\ \mathrm{C} & -3.278383 & -3.198221 & -0.183362 \\ \mathrm{C} & -2.259539 & -2.598790 & 0.499280 \\ \mathrm{C} & -1.123957 & -3.521346 & 0.900397 \\ \mathrm{H} & -0.233753 & -3.367897 & 0.281219 \\ \mathrm{H} & -1.413069 & -4.566573 & 0.780412 \\ \mathrm{H} & -0.825616 & -3.372084 & 1.942925 \\ \mathrm{H} & -3.165336 & -4.262004 & -0.376158 \\ \mathrm{H} & -4.951650 & 0.567852 & 0.290149 \\ \mathrm{O} & -2.946406 & 1.024060 & 1.163299 \\ \mathrm{C} & -1.841057 & 1.422537 & 1.989401 \\ \mathrm{C} & -2.158377 & 1.058289 & 3.438675 \\ \mathrm{H} & -2.342233 & -0.014687 & 3.544653 \\ \mathrm{H} & -3.056808 & 1.588672 & 3.767760 \\ \mathrm{H} & -1.330665 & 1.334781 & 4.099575 \\ \mathrm{H} & -0.653778 & -1.166475 & 2.466998 \\ \mathrm{H} & 0.031559 & -1.233628 & 0.860595 \\ \mathrm{H} & -0.506027 & 1.057902 & 0.404556 \\ \mathrm{C} & -6.993147 & -0.045845 & -0.795439 \\ \mathrm{H} & -7.965911 & -0.202525 & -1.261745 \\ \mathrm{H} & -7.130406 & 0.181350 & 0.269437 \\ \mathrm{H} & -6.485680 & 0.795869 & -1.283739 \\ \mathrm{C} & -1.811473 & 2.957670 & 1.810252 \\ \mathrm{C} & -1.415502 & 3.338135 & 0.415016 \\ \mathrm{C} & -0.323801 & 4.025519 & 0.087515 \\ \mathrm{C} & 0.219922 & 4.242864 & -1.308444 \\ \mathrm{C} & 0.320877 & 5.750189 & -1.595310 \\ \mathrm{H} & 0.897474 & 6.262450 & -0.816530 \\ \mathrm{H} & -0.674846 & 6.204075 & -1.631571 \\ \mathrm{H} & 0.815728 & 5.931854 & -2.556823 \\ \mathrm{C} & -0.652057 & 3.585312 & -2.384683 \\ \mathrm{H} & -1.669466 & 3.989635 & -2.371726 \\ \mathrm{H} & -0.725586 & 2.499674 & -2.252200 \\ \mathrm{H} & -0.230331 & 3.775535 & -3.377643 \\ \mathrm{C} & 1.656941 & 3.638705 & -1.366108 \\ \mathrm{C} & 1.748683 & 2.269670 & -0.712304 \\ \mathrm{C} & 2.348260 & 2.101303 & 0.529289 \\ \mathrm{C} & 3.297456 & 3.105983 & 1.100247 \\ \mathrm{H} & 4.135903 & 3.273438 & 0.415446 \\ \mathrm{C} & 3.695029 & 2.755070 & 2.052186 \\ & 2.798213 & 4.070125 & 1.257149 \\ & 1.998798 & 0.899094 & 1.369199\end{array}$




$\begin{array}{rrrr}\mathrm{O} & 3.001993 & 0.565221 & 2.289467 \\ \mathrm{H} & 3.811546 & 0.441025 & 1.757887 \\ \mathrm{C} & 0.704565 & 1.137118 & 2.182867 \\ \mathrm{H} & 0.816643 & 0.534624 & 3.090324 \\ \mathrm{H} & 0.671828 & 2.183220 & 2.504262 \\ \mathrm{H} & 1.815913 & 0.061699 & 0.668877 \\ \mathrm{H} & 0.887605 & 1.629496 & -0.893489 \\ \mathrm{H} & 2.353942 & 4.328621 & -0.881137 \\ \mathrm{H} & 1.961098 & 3.600470 & -2.419851 \\ \mathrm{H} & 0.301226 & 4.429920 & 0.888531 \\ \mathrm{H} & -2.048944 & 2.920155 & -0.364615 \\ \mathrm{H} & -2.821516 & 3.318384 & 2.040588 \\ \mathrm{H} & -1.133970 & 3.404156 & 2.545733 \\ \mathrm{C} & 2.891064 & 1.181937 & -1.919135 \\ \mathrm{C} & 2.922217 & -0.182964 & -1.567759 \\ \mathrm{C} & 3.904203 & -0.472423 & -0.545340 \\ \mathrm{C} & 4.284488 & -1.792280 & -0.053406 \\ \mathrm{C} & 3.693754 & -3.002979 & -0.165124 \\ \mathrm{O} & 4.189149 & -4.115824 & 0.388641 \\ \mathrm{C} & 2.384676 & -3.323843 & -0.830408 \\ \mathrm{O} & 1.888983 & -4.422950 & -0.619959 \\ \mathrm{C} & 1.707399 & -2.395145 & -1.725050 \\ \mathrm{C} & 1.910173 & -1.093262 & -2.067224 \\ \mathrm{C} & 0.924396 & -0.527609 & -3.072540 \\ \mathrm{H} & 0.370941 & 0.325951 & -2.663526 \\ \mathrm{H} & 0.191895 & -1.280353 & -3.368042 \\ \mathrm{H} & 1.436186 & -0.182420 & -3.977898 \\ \mathrm{H} & 0.862633 & -2.898800 & -2.187364 \\ \mathrm{H} & 5.182233 & -1.714985 & 0.550309 \\ \mathrm{O} & 4.491207 & 0.499592 & -0.001172 \\ \mathrm{H} & 3.805232 & 1.743453 & -1.760309 \\ \mathrm{H} & 2.325346 & 1.475817 & -2.796371 \\ \mathrm{C} & 5.409739 & -4.053108 & 1.094967 \\ \mathrm{H} & 5.615746 & -5.074199 & 1.415339 \\ \mathrm{H} & 6.224163 & -3.696300 & 0.452764 \\ \mathrm{H} & 5.326287 & -3.402501 & 1.974035\end{array}$




\section{TS-6}

$\begin{array}{lr}\text { E } & -1886.050100 \\ \mathrm{H} & -1885.268571 \\ \mathrm{G} & -1885.383450 \\ \text { Imag. Freq. } & -409.184\end{array}$

Cartesian coordinates

$\begin{array}{rrrr}\mathrm{C} & -1.475932 & 0.033461 & 1.115819 \\ \mathrm{C} & -1.913117 & -1.434493 & 1.188844 \\ \mathrm{C} & -3.250619 & -1.581474 & 0.500999 \\ \mathrm{C} & -4.164596 & -0.644647 & 0.906009 \\ \mathrm{C} & -5.526379 & -0.424297 & 0.528065 \\ \mathrm{C} & -6.307271 & -1.052081 & -0.391178 \\ \mathrm{O} & -7.581290 & -0.704941 & -0.615500 \\ \mathrm{C} & -5.923808 & -2.168561 & -1.304496 \\ \mathrm{O} & -6.742895 & -2.562094 & -2.126376 \\ \mathrm{C} & -4.620461 & -2.809417 & -1.220754 \\ \mathrm{C} & -3.486390 & -2.598679 & -0.489005 \\ \mathrm{C} & -2.346410 & -3.563798 & -0.756515 \\ \mathrm{H} & -1.476813 & -3.050422 & -1.183993 \\ \mathrm{H} & -2.650039 & -4.339574 & -1.461587 \\ \mathrm{H} & -2.015111 & -4.056600 & 0.163964 \\ \mathrm{H} & -4.591903 & -3.635707 & -1.926393 \\ \mathrm{H} & -5.960111 & 0.394635 & 1.090469 \\ \mathrm{O} & -3.802939 & 0.225224 & 1.896336 \\ \mathrm{C} & -2.523365 & 0.905576 & 1.846198 \\ \mathrm{C} & -2.205584 & 1.159456 & 3.315056 \\ \mathrm{H} & -3.067959 & 1.633974 & 3.791579 \\ \mathrm{H} & -1.339420 & 1.815102 & 3.434576 \\ \mathrm{H} & -2.003494 & 0.219446 & 3.837388 \\ \mathrm{C} & -2.778568 & 2.221385 & 1.072276 \\ \mathrm{C} & -1.537713 & 3.015597 & 0.780514 \\ \mathrm{C} & -1.017888 & 3.158558 & -0.438269 \\ \mathrm{C} & 0.245250 & 3.891246 & -0.828216 \\ \mathrm{C} & 0.944453 & 4.536517 & 0.373610 \\ \mathrm{H} & 1.200138 & 3.806522 & 1.148148 \\ \mathrm{H} & 0.305330 & 5.299349 & 0.830695 \\ \mathrm{H} & 1.871648 & 5.025604 & 0.053788 \\ \mathrm{C} & -0.138657 & 4.995316 & -1.832410 \\ \mathrm{H} & -0.634974 & 4.574113 & -2.714353 \\ \mathrm{H} & 0.750628 & 5.538821 & -2.172585 \\ \mathrm{H} & -0.823346 & 5.714371 & -1.371120 \\ \mathrm{C} & 1.220078 & 2.921469 & -1.561587 \\ \mathrm{H} & 0.729410 & 2.533585 & -2.462192 \\ \mathrm{H} & 2.057334 & 3.536348 & -1.914406 \\ \mathrm{C} & 1.732755 & 1.773274 & -0.706431 \\ \mathrm{H} & 2.214515 & 2.064771 & 0.224917 \\ \mathrm{C} & 1.055904 & 0.563428 & -0.630711 \\ \mathrm{C} & 0.171879 & 0.073284 & -1.736462 \\ \mathrm{H} & -0.700111 & 0.719082 & -1.881681 \\ \mathrm{H} & 0.731099 & 0.049084 & -2.677730 \\ \mathrm{H} & -0.177329 & -0.943655 & -1.543135 \\ \mathrm{C} & 1.085304 & -0.218041 & 0.677313 \\ \mathrm{O} & 2.261824 & 0.011754 & 1.424584 \\ \mathrm{C} & 2.987387 & -0.482599 & 1.024525 \\ \mathrm{H} & -0.043845 & 0.248696 & 1.613554 \\ & 0.109213 & -0.276688 & 2.563525\end{array}$




$\begin{array}{rrrr}\mathrm{H} & 0.146449 & 1.308840 & 1.804016 \\ \mathrm{H} & 0.977805 & -1.286154 & 0.448954 \\ \mathrm{H} & -1.557734 & 2.715993 & -1.279323 \\ \mathrm{H} & -1.035823 & 3.462341 & 1.637780 \\ \mathrm{H} & -3.270714 & 1.952119 & 0.129199 \\ \mathrm{H} & -3.492973 & 2.818701 & 1.652831 \\ \mathrm{H} & -1.988327 & -1.739744 & 2.242931 \\ \mathrm{H} & -1.149274 & -2.065339 & 0.734256 \\ \mathrm{H} & -1.525786 & 0.319569 & 0.062201 \\ \mathrm{C} & -8.168325 & 0.326563 & 0.146188 \\ \mathrm{H} & -8.160607 & 0.087645 & 1.216951 \\ \mathrm{H} & -7.658360 & 1.284401 & -0.016967 \\ \mathrm{H} & -9.198040 & 0.399997 & -0.203391 \\ \mathrm{C} & 3.414765 & 1.303325 & -1.717346 \\ \mathrm{C} & 4.262322 & 0.413134 & -1.038004 \\ \mathrm{C} & 3.776026 & -0.948505 & -1.073671 \\ \mathrm{C} & 4.421823 & -2.125328 & -0.492222 \\ \mathrm{C} & 5.600988 & -2.290186 & 0.150753 \\ \mathrm{O} & 6.043832 & -3.480520 & 0.572238 \\ \mathrm{C} & 6.602020 & -1.231688 & 0.533671 \\ \mathrm{O} & 7.663538 & -1.593682 & 1.017613 \\ \mathrm{C} & 6.314793 & 0.193301 & 0.415631 \\ \mathrm{C} & 5.359890 & 0.911876 & -0.231799 \\ \mathrm{C} & 5.481611 & 2.419198 & -0.112986 \\ \mathrm{H} & 6.270541 & 2.691326 & 0.590350 \\ \mathrm{H} & 4.546549 & 2.868149 & 0.240827 \\ \mathrm{H} & 5.725338 & 2.877247 & -1.078637 \\ \mathrm{H} & 7.088747 & 0.755457 & 0.931006 \\ \mathrm{H} & 3.808251 & -3.001212 & -0.672791 \\ \mathrm{O} & 2.644544 & -1.147732 & -1.579013 \\ \mathrm{H} & 3.735431 & 2.332065 & -1.828464 \\ \mathrm{H} & 2.870802 & 0.903336 & -2.566753 \\ \mathrm{C} & 5.271751 & -4.635766 & 0.325353 \\ \mathrm{H} & 5.842304 & -5.464552 & 0.744066 \\ \mathrm{H} & 5.125618 & -4.794462 & -0.749877 \\ \mathrm{H} & 4.294688 & -4.575556 & 0.820631\end{array}$




\section{Computational Methods for DFT-GIAO NMR Predictions}

All calculations were carried out in ORCA 4.2.1 release version. ${ }^{19}$ Geometry optimizations were carried

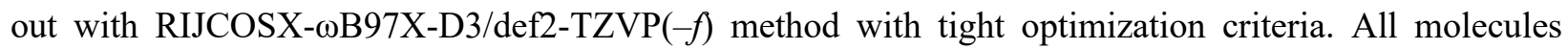
studied were verified to be stationary points by means of analytical frequency calculations

Sample input for optimization:

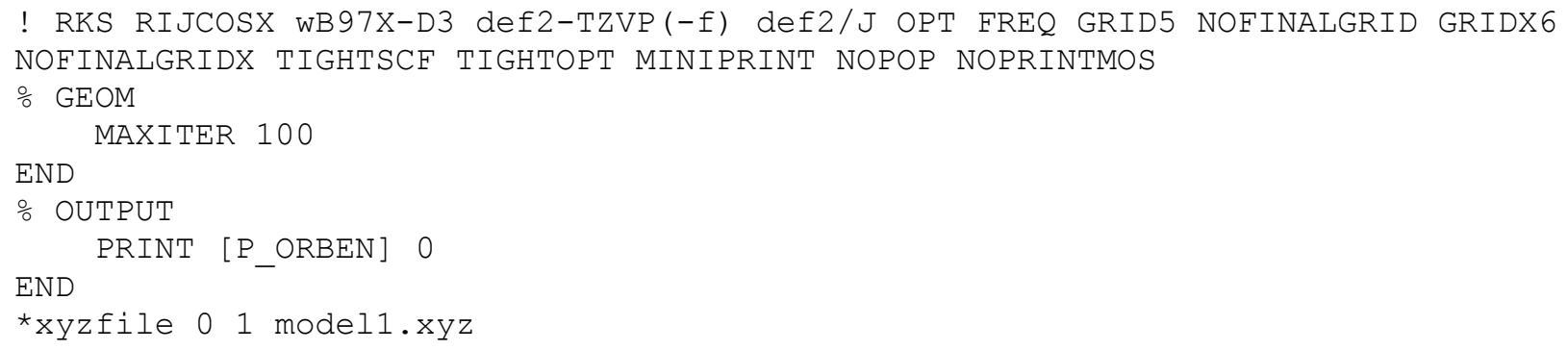

Isotropic shieldings were computed with GIAO approach and implicit solvation with CPCM model, with PBE0 / pcSseg-2. ${ }^{20}$ Auxiliary basis set was generated with ORCA autoaux procedure.

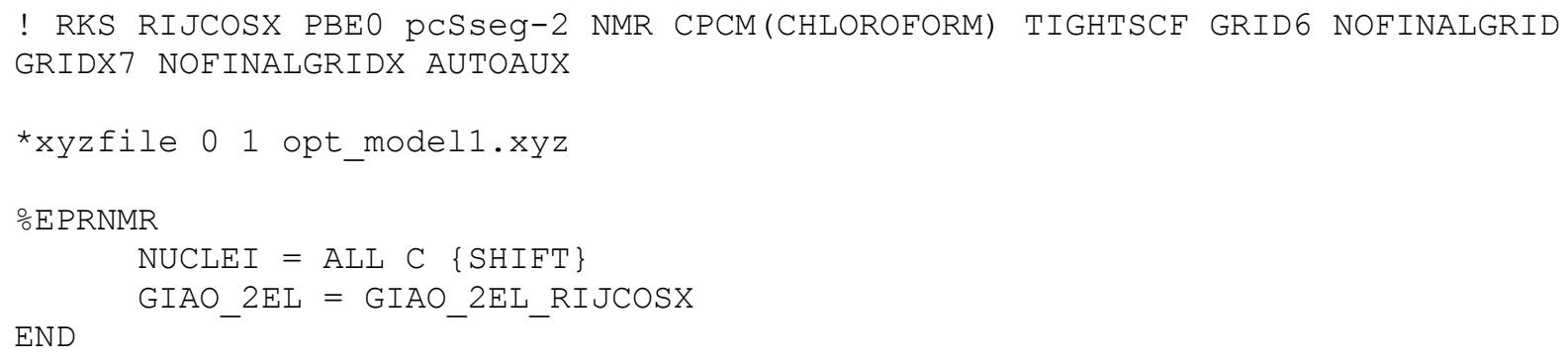

The following statistics were used for the purposes of NMR analysis:

$$
\begin{aligned}
\text { MAE } & =\frac{1}{N} \sum_{i=1}^{N}\left|\delta_{i}^{\mathrm{pred}}-\delta_{i}^{\mathrm{obs}}\right| \\
\mathrm{RMSE} & =\sqrt{\frac{1}{N} \sum_{i=1}^{N}\left(\delta_{i}^{\mathrm{pred}}-\delta_{i}^{\mathrm{obs}}\right)^{2}} \\
\operatorname{MAX} & =\max _{i=1 \ldots N}\left|\delta_{i}^{\mathrm{pred}}-\delta_{i}^{\mathrm{obs}}\right|
\end{aligned}
$$

$N$, total number of resonances compared; $\delta_{i}$ is the corresponding chemical shift, MAE, mean absolute error; RMS, root-mean square error; MAX, maximum absolute error.

According to a popular scheme, conversion from isotropic shieldings $\sigma i$ to chemical shifts $\delta i$ was carried out using a linear regression on a small molecule calibration set: 


$$
\begin{gathered}
k, b=\operatorname{argmin}\left\{\sum_{i=1}^{N}\left[\delta_{i}^{\mathrm{obs}}-\left(k \sigma_{i}^{\mathrm{calcd}}+b\right)\right]^{2}\right\} \\
\delta_{i}^{\mathrm{pred}}=k \sigma_{i}^{\text {calcd }}+b
\end{gathered}
$$

Linear correction parameters were determined to be:

$$
\delta_{i}^{\text {pred }}=k \sigma_{i}^{\text {calcd }}+b=-0.9127 \sigma_{i}^{\text {calcd }}+170.87
$$

Cartesian coordinates of deoxypycnidione (in $\AA$ ):

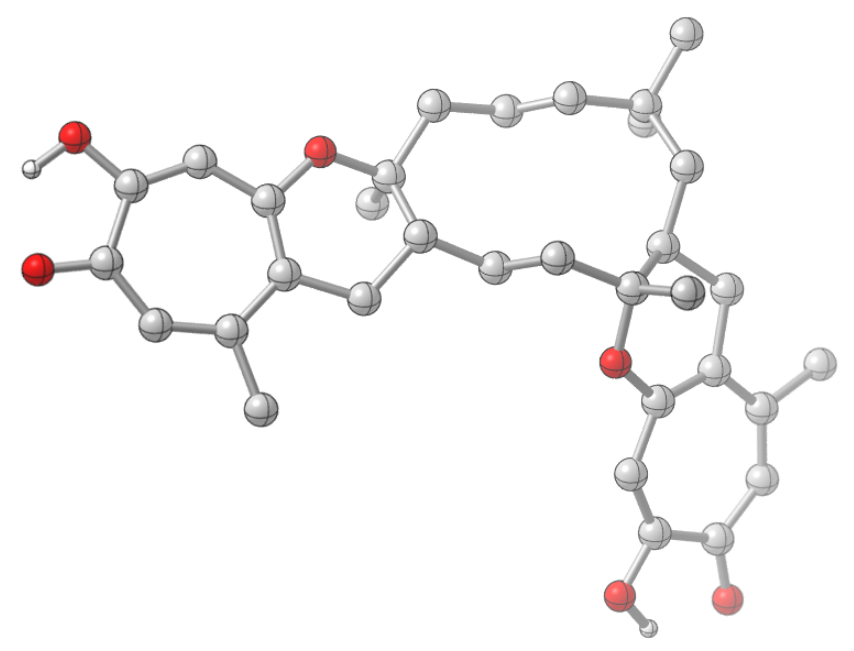

$\begin{array}{rrrr}\mathrm{H} & 3.748850 & 9.901200 & 3.251170 \\ \mathrm{O} & 4.773710 & 11.830950 & 4.693350 \\ \mathrm{O} & -4.556650 & 6.790740 & 4.782060 \\ \mathrm{O} & 1.015410 & 6.545430 & 5.791720 \\ \mathrm{O} & 3.618270 & 16.084610 & 5.649190 \\ \mathrm{H} & 3.986170 & 16.912470 & 6.036460 \\ \mathrm{C} & 5.382820 & 12.861670 & 5.322220 \\ \mathrm{C} & 5.679330 & 7.120390 & 5.871310 \\ \mathrm{H} & 5.612430 & 6.729320 & 4.855440 \\ \mathrm{C} & 7.234550 & 8.951670 & 5.248770 \\ \mathrm{H} & 8.292830 & 9.221870 & 5.321340 \\ \mathrm{H} & 7.098590 & 8.594640 & 4.224380 \\ \mathrm{C} & 7.322740 & 11.401480 & 5.786530 \\ \mathrm{H} & 7.824030 & 11.185030 & 6.731330 \\ \mathrm{H} & 8.119150 & 11.430100 & 5.032890 \\ \mathrm{C} & 4.295790 & 9.611610 & 4.154160 \\ \mathrm{H} & 4.721050 & 8.625070 & 3.956320 \\ \mathrm{C} & 3.318480 & 9.546460 & 5.323450\end{array}$




\begin{tabular}{|c|c|c|c|}
\hline & 2.890550 & 10.539450 & 5.4666 \\
\hline & 3.861800 & 9.324430 & 6.239020 \\
\hline & 2.179220 & 8.528850 & 5.125810 \\
\hline & 2.322890 & 8.010790 & \\
\hline & 6.396070 & 10.225780 & 5.488660 \\
\hline & 5.763580 & 10.081600 & 6.367390 \\
\hline & 2.096570 & 7.418720 & 6.179250 \\
\hline & 6.640640 & 12.749040 & \\
\hline & -3.362730 & 7.088660 & 4.915320 \\
\hline & 7.014120 & 7.743370 & 6.193220 \\
\hline & 7.148660 & 8.156070 & 7.657450 \\
\hline & 7.055050 & 7.289240 & 8.314710 \\
\hline & 6.394390 & 8.88 & \\
\hline & 8.130290 & 8.602950 & 7.833340 \\
\hline & -0.201120 & 7.041690 & 5.449820 \\
\hline & 4.600590 & 7.033080 & 6.634100 \\
\hline & 4.631590 & 7.396980 & 7.658050 \\
\hline & 5.447620 & 10.605060 & 4.343370 \\
\hline & -2.892080 & 8.424930 & 4.767980 \\
\hline & -3.708270 & 9.102120 & 4.542920 \\
\hline & -1.140020 & 5.972440 & 5.480410 \\
\hline & -0.701520 & 5.016930 & 5.743320 \\
\hline & 7.360380 & 13.820030 & \\
\hline & 4.481760 & 13.964580 & 5.313700 \\
\hline & 3.533000 & 13.744310 & 4.838550 \\
\hline & 5.793050 & 15.843610 & 6.429830 \\
\hline & 0.841540 & & \\
\hline & 0.804890 & 9.974730 & 5.903440 \\
\hline & 0.825360 & 9.861770 & 4.166670 \\
\hline & 6.988450 & 15.120730 & 6.709630 \\
\hline & 7.721310 & 15.756390 & 7.193360 \\
\hline & 5.658430 & 17.039880 & 6.717530 \\
\hline & -0.379160 & 8.365940 & 5.118750 \\
\hline & 1.809530 & 7.931580 & 7.586990 \\
\hline & 0.832230 & 8.411890 & 7.639010 \\
\hline & 2.557880 & 8.657410 & 7.907060 \\
\hline & 1.810150 & 7.100120 & 8.292760 \\
\hline & -3.137970 & 4.813980 & 5.325220 \\
\hline & -4.065390 & 5.085220 & 5.133140 \\
\hline & -2.469660 & 5.959850 & 5.243140 \\
\hline & 8.103110 & 6.710180 & 5.867390 \\
\hline & 9.097890 & 7.125160 & 6.046450 \\
\hline & 8.051020 & 6.401810 & 4.820290 \\
\hline & 7.988460 & 5.818360 & 6.486420 \\
\hline & 3.299180 & 6.460740 & 6.157300 \\
\hline
\end{tabular}




$\begin{array}{rrrr}\mathrm{H} & 3.019990 & 5.604820 & 6.779490 \\ \mathrm{H} & 3.411420 & 6.086770 & 5.136610 \\ \mathrm{C} & -1.641670 & 8.979630 & 4.833460 \\ \mathrm{C} & 6.165070 & 10.840920 & 3.017930 \\ \mathrm{H} & 5.448080 & 11.202280 & 2.280630 \\ \mathrm{H} & 6.608060 & 9.920170 & 2.637640 \\ \mathrm{H} & 6.954250 & 11.587480 & 3.114880 \\ \mathrm{C} & 4.621730 & 15.223480 & 5.779740 \\ \mathrm{C} & -1.609760 & 10.469640 & 4.563260 \\ \mathrm{H} & -1.088110 & 10.687270 & 3.628430 \\ \mathrm{H} & -1.091350 & 11.012800 & 5.354720 \\ \mathrm{H} & -2.618370 & 10.867430 & 4.483020 \\ \mathrm{C} & 8.754340 & 13.486930 & 6.979580 \\ \mathrm{H} & 8.722910 & 12.758570 & 7.793060 \\ \mathrm{H} & 9.254780 & 14.378040 & 7.350440 \\ \mathrm{H} & 9.365090 & 13.056540 & 6.183770\end{array}$

Computed chemical shift statistics:

$\begin{array}{lrrrrrr}\text { Nucleus } & \sigma^{\text {calcd }} / \mathrm{ppm} & \delta^{\text {pred }} / \text { ppm } & \text { Exp NMR 1 } & \text { Exp NMR 2 } & \text { Error 1 } & \text { Error 2 } \\ 43 & 1.845 & 169.2 & 172.72 & 172.76 & -3.5 & -3.6 \\ 26 & 1.851 & 169.2 & 172.02 & 172.37 & -2.8 & -3.2 \\ 57 & 13.955 & 158.1 & 164.04 & 163.16 & -5.9 & -5.0 \\ 70 & 14.081 & 158.0 & 163.23 & 160.69 & -5.2 & -2.7 \\ 6 & 14.909 & 157.3 & 160.72 & 160.66 & -3.5 & -3.4 \\ 32 & 15.401 & 156.8 & 160.66 & 150.49 & -3.8 & 6.3 \\ 65 & 20.569 & 152.1 & 149.99 & 150.49 & 2.1 & 1.6 \\ 40 & 20.633 & 152.0 & 149.29 & 149.72 & 2.7 & 2.3 \\ 7 & 30.112 & 143.4 & 143.67 & 144.51 & -0.3 & -1.1 \\ 36 & 51.854 & 123.5 & 124.56 & 133.64 & -1.0 & -10.1 \\ 47 & 52.699 & 122.8 & 124.2 & 133.53 & -1.4 & -10.8 \\ 33 & 52.746 & 122.7 & 121.77 & 124.81 & 1.0 & -2.1 \\ 50 & 57.279 & 118.6 & 121.31 & 124.5 & -2.7 & -5.9 \\ 25 & 59.304 & 116.7 & 120.28 & 121.08 & -3.5 & -4.3 \\ 38 & 67.328 & 109.4 & 113.67 & 113.48 & -4.3 & -4.1 \\ 41 & 67.846 & 108.9 & 112.98 & 113.15 & -4.0 & -4.2 \\ 24 & 100.158 & 79.5 & 82.01 & 81.29 & -2.6 & -1.8 \\ 35 & 100.294 & 79.3 & 80.77 & 80.36 & -1.4 & -1.0 \\ 62 & 132.512 & 49.9 & 48.51 & 45.54 & 1.4 & 4.4 \\ 9 & 134.823 & 47.8 & 46.86 & 36.29 & 1.0 & 11.5 \\ 20 & 142.672 & 40.7 & 40.92 & 33.95 & -0.3 & 6.7 \\ 15 & 144.081 & 39.4 & 37.95 & 32.08 & 1.4 & 7.3 \\ 27 & 144.464 & 39.0 & 36.82 & 31.41 & 2.2 & 7.6 \\ 44 & 147.565 & 36.2 & 34.05 & 30.11 & 2.1 & 6.1 \\ 12 & 148.262 & 35.6 & 32.48 & 29.85 & 3.1 & 5.7 \\ & & & 581 & & & \\ & & & & & & \end{array}$




\begin{tabular}{|c|c|c|c|c|c|c|}
\hline 58 & 152.335 & 31.8 & 31.39 & 29.52 & 0.4 & 2.3 \\
\hline 22 & 153.454 & 30.8 & 30.28 & 27.41 & 0.5 & 3.4 \\
\hline 75 & 154.325 & 30.0 & 29.85 & 27.25 & 0.2 & 2.8 \\
\hline 71 & 154.515 & 29.8 & 27.4 & 23.81 & 2.4 & 6.0 \\
\hline 17 & 157.087 & 27.5 & 27.32 & 22.85 & 0.2 & 4.6 \\
\hline 28 & 163.637 & 21.5 & 24.82 & 21.71 & -3.3 & -0.2 \\
\hline 66 & 163.875 & 21.3 & 21.24 & 19.54 & 0.1 & 1.8 \\
\hline 51 & 168.618 & 17.0 & 18.92 & 14.28 & -1.9 & 2.7 \\
\hline & & \multirow{3}{*}{\multicolumn{2}{|c|}{ All atoms: }} & MAE & 2.2 & 4.4 \\
\hline & & & & RMSE & 2.7 & 5.2 \\
\hline & & & & MAX & 5.9 & 11.5 \\
\hline & & \multirow{3}{*}{\multicolumn{2}{|c|}{ Aliphatic only: }} & MAE & 1.4 & 4.5 \\
\hline & & & & RMSE & 1.8 & 5.3 \\
\hline & & & & MAX & 3.3 & 11.5 \\
\hline
\end{tabular}

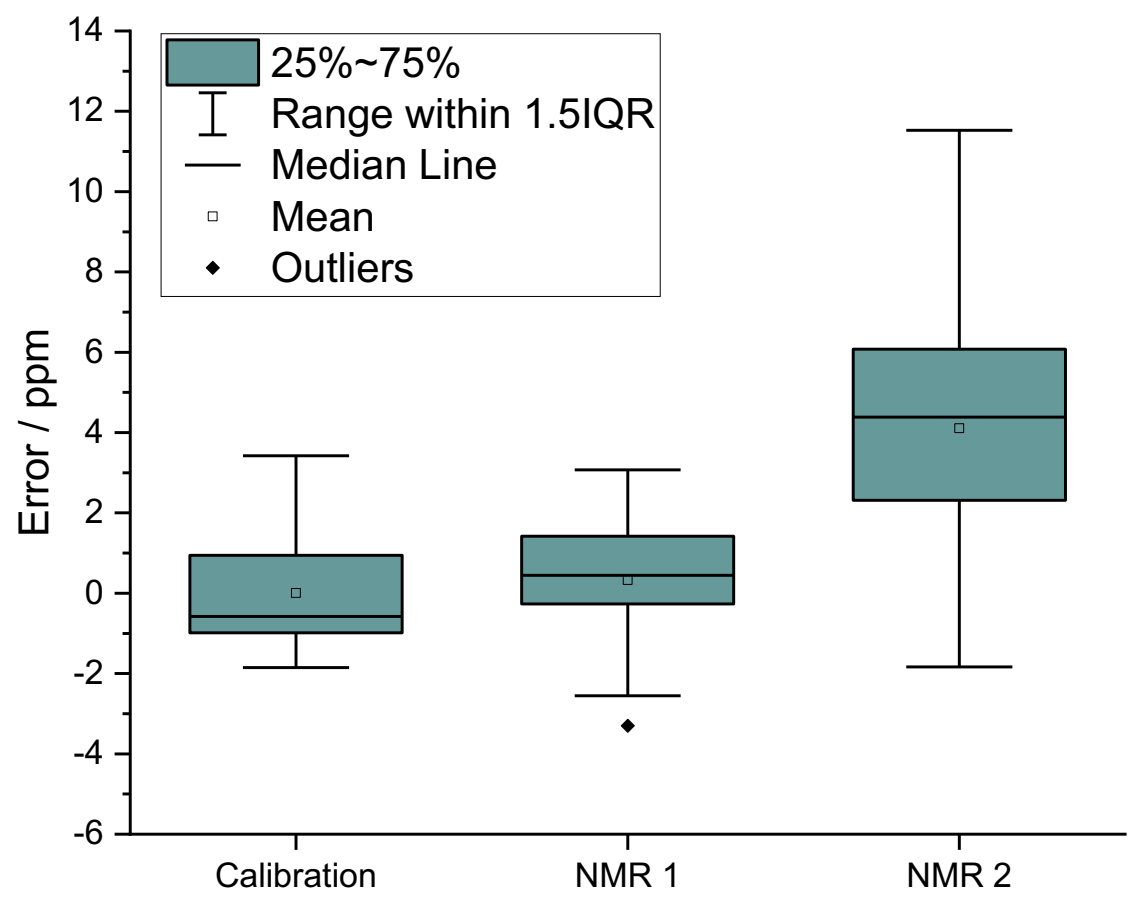

Figure S18. Error distribution comparison for calibration and two models (only aliphatic atoms included). 

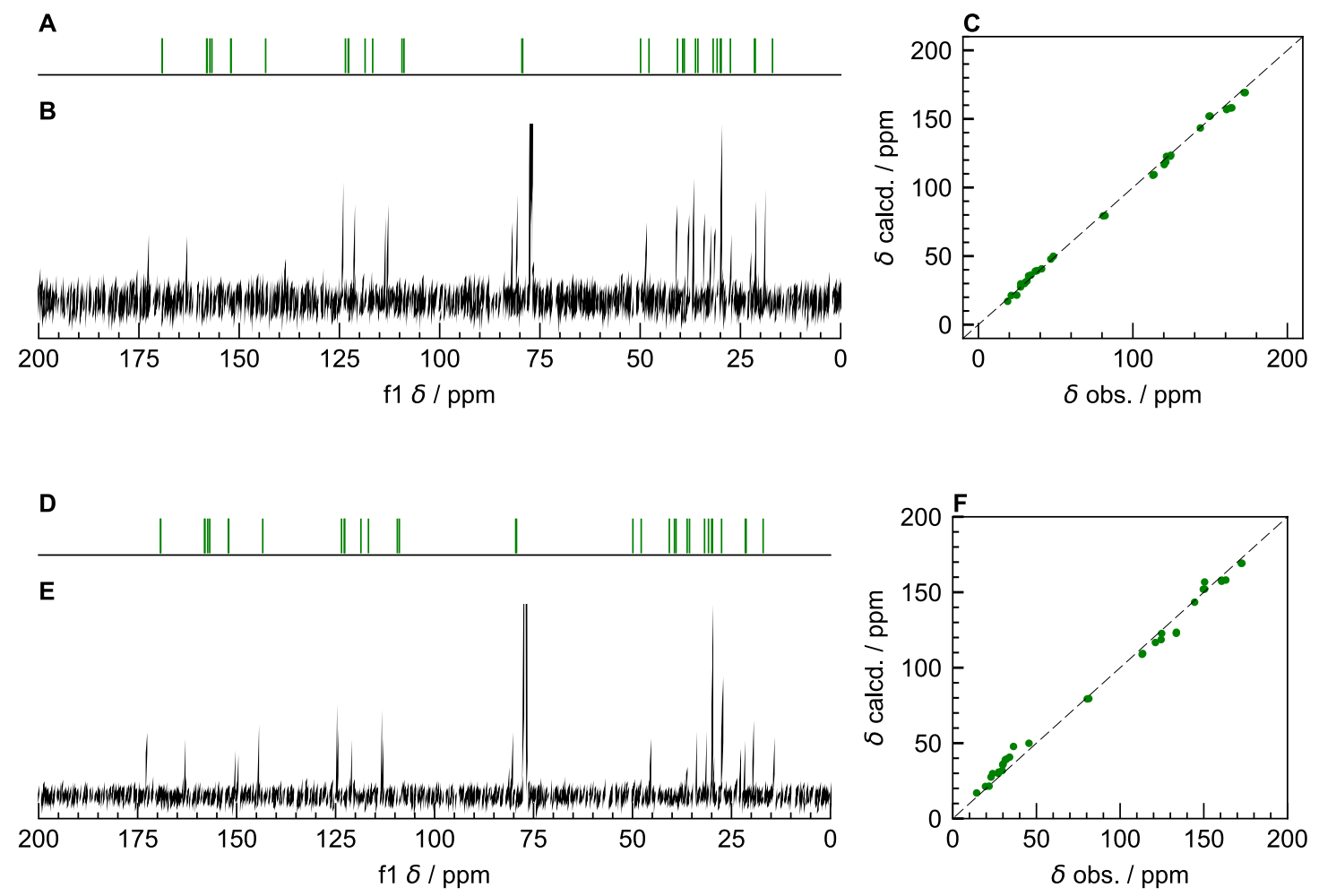

Figure S19. A and D, predicted 13C NMR spectra of deoxypycnidione. B, experimental spectrum of deoxypycnidione. E, experimental spectrum of [diastereomer of deoxypycnidione]. $\mathrm{C}, \mathrm{F}$, scatter plots of predicted/observed $13 \mathrm{C}$ chemical shifts for corresponding experimental spectra. Dashed diagonal line: $y=x$.

\section{Computational Methods for TDDFT-ECD Predictions}

Calculations were performed with ORCA 4.2.1 release version. Two geometries were used: one was taken directly from our X-ray crystallographic experiment, the other one was optimized from the coordinates found in the previously reported X-ray experiment using PBEh- $3 \mathrm{c}^{21}$ method.

Time-Dependent Density Functional Theory (TDDFT) with RIJCOSX- $\omega$ B97X/def2-TZVP was used to compute vertical excitation energies, as well as UV/Vis and ECD intensities. Example of the input:

! RKS RIJCOSX wB97X def2-TZVP def2/J TIGHTSCF GRID5 NOFINALGRID GRIDX 6 NOFINALGRIDX CPCM(Ethanol)

TDDFT

NROOTS 15

TDA FALSE

TRIPLETS FALSE

END

*xyzfile 01 pyc.xyz

In order to bring transition energies (not wavelengths!) in closer correspondence to experiment, they were empirically shifted by $+5627.4 \mathrm{~cm}^{-1}$. This number was obtained in a way that the 
maximum for $\pi \rightarrow \pi^{*}$ transition maxima in the wavelength domain would match the reported experimental value of $255 \mathrm{~nm}$. The predicted line of highest intensity. The following wavelength shifts were therefore obtained:

$$
\begin{aligned}
& 302.1 \mathrm{~nm} \rightarrow 363.9 \mathrm{~nm} \\
& 301.1 \mathrm{~nm} \rightarrow 362.5 \mathrm{~nm} \\
& 300.7 \mathrm{~nm} \rightarrow 362.0 \mathrm{~nm} \\
& 299.7 \mathrm{~nm} \rightarrow 360.5 \mathrm{~nm} \\
& 284.6 \mathrm{~nm} \rightarrow 338.8 \mathrm{~nm} \\
& 281.7 \mathrm{~nm} \rightarrow 334.7 \mathrm{~nm} \\
& 226.1 \mathrm{~nm} \rightarrow 259.1 \mathrm{~nm} \\
& 223.1 \mathrm{~nm} \rightarrow 255.2 \mathrm{~nm} \\
& 236.2 \mathrm{~nm} \rightarrow 272.4 \mathrm{~nm} \\
& 230.3 \mathrm{~nm} \rightarrow 264.5 \mathrm{~nm} \\
& 214.6 \mathrm{~nm} \rightarrow 244.1 \mathrm{~nm} \\
& 210.9 \mathrm{~nm} \rightarrow 239.3 \mathrm{~nm} \\
& 190.1 \mathrm{~nm} \rightarrow 212.8 \mathrm{~nm} \\
& 188.7 \mathrm{~nm} \rightarrow 211.1 \mathrm{~nm} \\
& 181.4 \mathrm{~nm} \rightarrow 202.1 \mathrm{~nm}
\end{aligned}
$$

For plotting of the spectrum, gaussian lineshape with $\sigma=10$ (FWHM of $24 \mathrm{~nm}$ ) was used
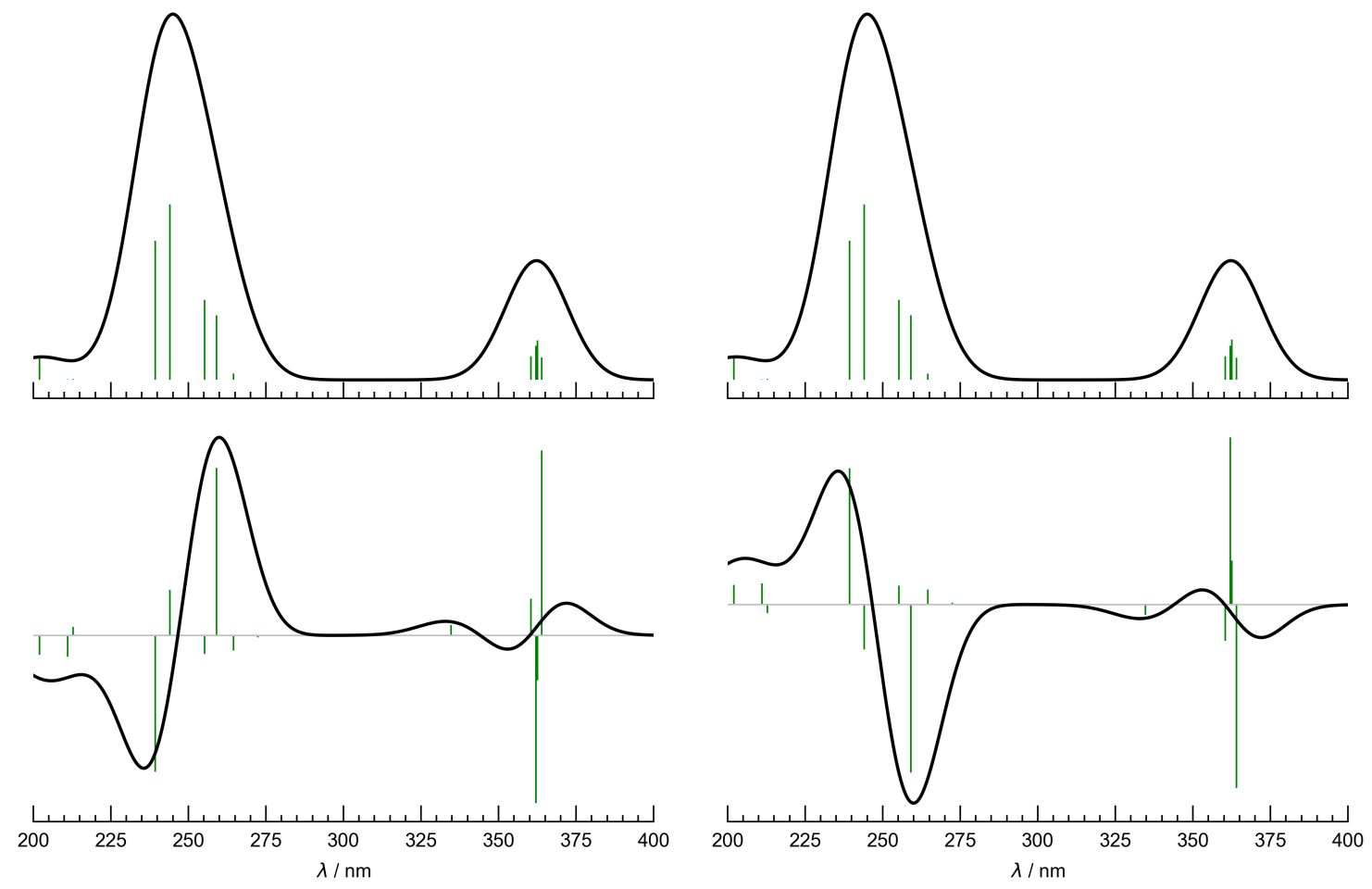

Figure S20. (Left): computed ECD and UV spectrum of pycnidione, geometry obtained experimentally from X-ray diffraction experiment ( $P 6_{1}$ space group).

(Right): same, with inverted absolute stereochemistry (as from $P 6_{5}$ space group). Left spectrum matches the original report perfectly:

Reported: $\quad \mathrm{CD}(\mathrm{EtOH}): 260 \mathrm{~nm}(+63), 238 \mathrm{~nm}(-15)$.

Calculated: $\quad \mathrm{CD}(\mathrm{EtOH}): 260 \mathrm{~nm}(+), 240 \mathrm{~nm}(-)$ 
Cartesian coordinates for pycnidione (in $\AA$ )

\begin{tabular}{|c|c|c|c|}
\hline & 3.586800 & 9.832300 & 3100 \\
\hline & 3.527200 & 9.114200 & 2.381100 \\
\hline & 4.860800 & 11.856700 & 4.503100 \\
\hline & -4.461800 & 6.546200 & 4.600200 \\
\hline & 0.966000 & 6.936500 & 6.292500 \\
\hline & 3.404200 & 15.641200 & 6.455100 \\
\hline & 3.538200 & 16.276000 & 6.974300 \\
\hline & 5.395800 & 12.7 & \\
\hline & 5.636200 & & \\
\hline & 5.458100 & 6.646900 & 5.253600 \\
\hline & 7.203700 & 8.847500 & 5.005300 \\
\hline & 8.154100 & 9.070400 & 4.929600 \\
\hline & 6.915600 & & \\
\hline & 7.384800 & 11.298200 & 5.600400 \\
\hline & 7.922900 & 11.053400 & 6.383700 \\
\hline & 7.995500 & 11.423300 & 4.844300 \\
\hline & 4.274200 & 9.616900 & 4.023400 \\
\hline & 4.618100 & 8.689000 & \\
\hline & 3.281400 & 9.757200 & 5.167200 \\
\hline & 2.873200 & 10.647300 & 5.124900 \\
\hline & 3.766000 & 9.692000 & 6.016300 \\
\hline & 2.164400 & 8.696500 & 5.149400 \\
\hline & 2.348100 & 8.078900 & 4.3 \\
\hline & 6.419200 & 10.149400 & 5.278100 \\
\hline & 5.865400 & 9.995200 & 6.082900 \\
\hline & 2.088700 & 7.865900 & 6.426000 \\
\hline & 6.677600 & $12.5 \mathrm{~s}$ & 5.877700 \\
\hline & -3.274300 & 6.93 & 4.80 \\
\hline & 7.045000 & 7.737400 & 6.074000 \\
\hline & 7.397400 & 8.260600 & 7.470400 \\
\hline & 7.336100 & 7.535500 & 8.112100 \\
\hline & 6.779100 & 8.965500 & 7.716800 \\
\hline & 8.302700 & 8.610900 & 7.465700 \\
\hline & -0.207700 & 7.278200 & 5.695600 \\
\hline & 4.618900 & 7.420500 & 6.799600 \\
\hline & 4.757900 & 7.95 & 7.557200 \\
\hline & 5.481200 & 10.576700 & 4.155700 \\
\hline & -2.846600 & 8.214300 & 4.412900 \\
\hline & -3.534900 & 8.727200 & 4.025800 \\
\hline & -1.098900 & 6.203800 & 5.889200 \\
\hline & -0.737600 & 5.487600 & 6.379000 \\
\hline & 7.379500 & 13.593600 & 6.625800 \\
\hline & 4.438700 & 13.769000 & 5.603600 \\
\hline & 3.622900 & 13.658000 & 5.148200 \\
\hline
\end{tabular}




\begin{tabular}{lrrr}
$\mathrm{C}$ & 5.620700 & 15.385000 & 7.143000 \\
$\mathrm{C}$ & 0.813900 & 9.367300 & 4.879400 \\
$\mathrm{H}$ & 0.713400 & 10.124800 & 5.493900 \\
$\mathrm{H}$ & 0.820100 & 9.726300 & 3.966900 \\
$\mathrm{C}$ & 6.891300 & 14.778600 & 7.137200 \\
$\mathrm{H}$ & 7.546400 & 15.290200 & 7.575800 \\
$\mathrm{O}$ & 5.397200 & 16.427600 & 7.813700 \\
$\mathrm{C}$ & -0.382300 & 8.466900 & 5.030200 \\
$\mathrm{C}$ & 1.861900 & 8.673600 & 7.685700 \\
$\mathrm{H}$ & 1.079900 & 9.234400 & 7.574200 \\
$\mathrm{H}$ & 2.636600 & 9.230600 & 7.856400 \\
$\mathrm{H}$ & 1.723900 & 8.073200 & 8.434600 \\
$\mathrm{O}$ & -2.916300 & 4.825000 & 5.822500 \\
$\mathrm{H}$ & -3.659300 & 4.736400 & 5.464400 \\
$\mathrm{C}$ & -2.401200 & 6.011200 & 5.491200 \\
$\mathrm{C}$ & 8.018300 & 6.594500 & 5.716000 \\
$\mathrm{H}$ & 8.924700 & 6.938600 & 5.686100 \\
$\mathrm{H}$ & 7.782000 & 6.229300 & 4.849000 \\
$\mathrm{H}$ & 7.960300 & 5.897600 & 6.388300 \\
$\mathrm{C}$ & 3.246900 & 6.846700 & 6.574000 \\
$\mathrm{H}$ & 3.037300 & 6.252200 & 7.324600 \\
$\mathrm{H}$ & 3.276200 & 6.296100 & 5.763600 \\
$\mathrm{C}$ & -1.631100 & 8.892900 & 4.468000 \\
$\mathrm{C}$ & 6.187600 & 10.774800 & 2.816100 \\
$\mathrm{H}$ & 5.570400 & 11.165900 & 2.179600 \\
$\mathrm{H}$ & 6.496200 & 9.917000 & 2.483400 \\
$\mathrm{H}$ & 6.947500 & 11.366100 & 2.934500 \\
$\mathrm{C}$ & 4.509100 & 14.881800 & 6.380000 \\
$\mathrm{C}$ & -1.644600 & 10.262700 & 3.866200 \\
$\mathrm{H}$ & -1.088800 & 10.271600 & 3.069400 \\
$\mathrm{H}$ & -1.299000 & 10.902800 & 4.508000 \\
$\mathrm{H}$ & -2.554000 & 10.502400 & 3.627400 \\
$\mathrm{C}$ & 8.830500 & 13.314600 & 6.902800 \\
$\mathrm{H}$ & 8.904900 & 12.586400 & 7.540100 \\
& 9.247200 & 14.109900 & 7.270400 \\
\hline
\end{tabular}




\section{Crystallographic Data}

Single crystal of $\mathrm{C}_{10} \mathrm{H}_{9} \mathrm{BO}_{4} \mathrm{~F}_{2} 19$ was selected and mounted using Cryo-loop (Hampton research) using Paratone-N oil (Exxon) with $\left(\begin{array}{lll}0 & 2 & -1\end{array}\right)$ face roughly perpendicular to the spindle axis on a Bruker APEX-II CCD diffractometer. The crystal was kept at $100.0 \mathrm{~K}$ during data collection. Using Olex $2,{ }^{22}$ the structure was solved with the SHELXT ${ }^{23}$ structure solution program using Intrinsic Phasing and refined with the $\mathrm{XL}^{24}$ refinement package using Least Squares minimisation.

Table S2. $\mathrm{BF}_{2}$-tropolone 19.

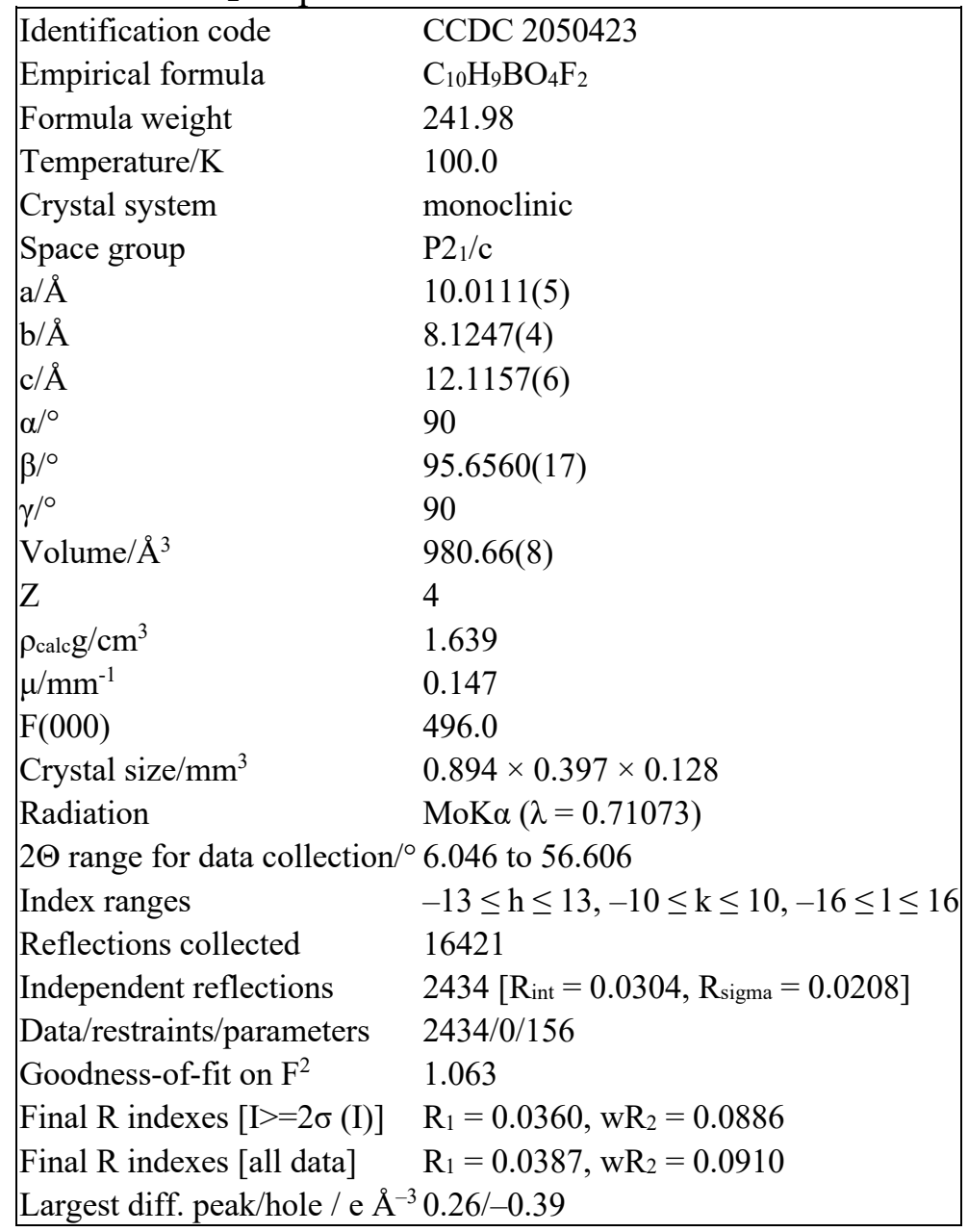

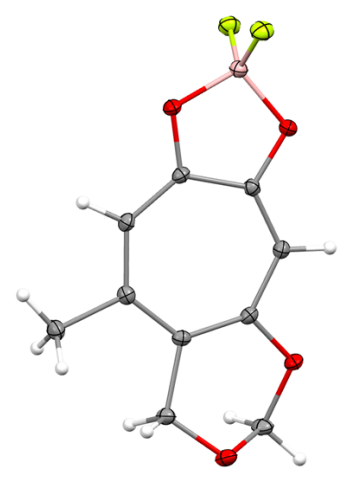


Single crystal of $\mathrm{C}_{11} \mathrm{H}_{12} \mathrm{O}_{4} \mathbf{2 0}$ was selected and Mounted on a Cryo-Loop (Hampton Research) with Paratone-N oil (Exxon) with (-1 4 2) face roughly perpendicular to the spindle axis on a Mo Bruker D8 Venture/Photon 100 diffractometer. The crystal was kept at $100.03 \mathrm{~K}$ during data collection. Using Olex2, ${ }^{22}$ the structure was solved with the $\mathrm{XT}^{23}$ structure solution program using Intrinsic Phasing and refined with the $\mathrm{XL}^{24}$ refinement package using Least Squares minimisation.

Table S3. Methoxy-tropolone 20.

\begin{tabular}{|c|c|}
\hline Identification code & CCDC 2050424 \\
\hline Empirical formula & $\mathrm{C}_{11} \mathrm{H}_{12} \mathrm{O}_{4}$ \\
\hline Formula weight & 208.21 \\
\hline Temperature/K & 100.03 \\
\hline Crystal system & triclinic \\
\hline Space group & $\mathrm{P}-1$ \\
\hline $\mathrm{a} / \AA$ & $7.8779(3)$ \\
\hline $\mathrm{b} / \AA$ & $10.6084(4)$ \\
\hline $\mathrm{c} / \AA$ & $12.5217(4)$ \\
\hline$\alpha /^{\circ}$ & $92.0813(9)$ \\
\hline$\beta /{ }^{\circ}$ & 107.3012(9) \\
\hline$\gamma /{ }^{\circ}$ & $109.5163(8)$ \\
\hline Volume $/ \AA^{3}$ & $930.95(6)$ \\
\hline Z & 4 \\
\hline$\rho_{\text {calc }} \mathrm{g} / \mathrm{cm}^{3}$ & 1.486 \\
\hline$\mu / \mathrm{mm}^{-1}$ & 0.113 \\
\hline $\mathrm{F}(000)$ & 440.0 \\
\hline Crystal size $/ \mathrm{mm}^{3}$ & $0.528 \times 0.39 \times 0.212$ \\
\hline Radiation & $\operatorname{MoK} \alpha(\lambda=0.71073)$ \\
\hline \multicolumn{2}{|c|}{$2 \Theta$ range for data collection $/{ }^{\circ} 4.958$ to 56.742} \\
\hline Index ranges & $-10 \leq \mathrm{h} \leq 10,-14 \leq \mathrm{k} \leq 14,-16 \leq 1 \leq 16$ \\
\hline Reflections collected & 52758 \\
\hline Independent reflections & $52758\left[\mathrm{R}_{\text {int }}=?, \mathrm{R}_{\text {sigma }}=0.0482\right]$ \\
\hline Data/restraints/parameters & $52758 / 0 / 277$ \\
\hline Goodness-of-fit on $\mathrm{F}^{2}$ & 1.048 \\
\hline Final R indexes $[\mathrm{I}>=2 \sigma(\mathrm{I})]$ & $\mathrm{R}_{1}=0.0445, \mathrm{wR}_{2}=0.1049$ \\
\hline Final R indexes [all data] & $\mathrm{R}_{1}=0.0575, \mathrm{wR}_{2}=0.1123$ \\
\hline Largest diff. peak/hole / e $\AA^{-3}$ & $30.41 /-0.23$ \\
\hline
\end{tabular}

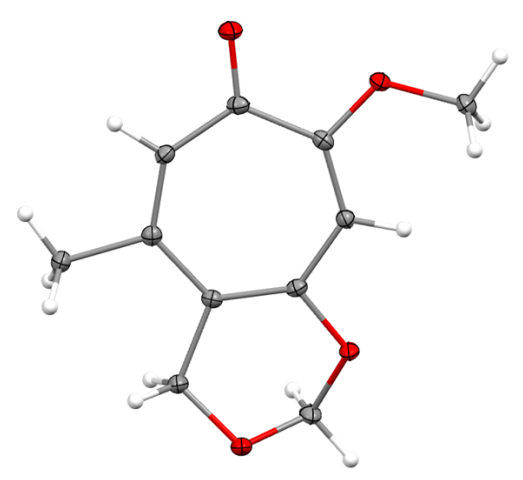


Single crystals of $\mathrm{C}_{15} \mathrm{H}_{24} \mathrm{O}_{2} 42$ were recrystallized from dichloromethane/ethanol co-solvent system. A suitable crystal was selected and the crystal was mounted on a loop with (2 12 2 $)^{2}$ face roughly perpendicular to the spindle axis. on a Bruker D8 Venture/Photon 100 diffractometer. The crystal was kept at $100 \mathrm{~K}$ during data collection. Using Olex $2,{ }^{22}$ the structure was solved with the SHELXT $^{23}$ structure solution program using Intrinsic Phasing and refined with the $\mathrm{XL}^{24}$ refinement package using Least Squares minimisation.

Table S4. Epoxide 42.

\begin{tabular}{|c|c|}
\hline Identification code & CCDC 2050426 \\
\hline Empirical formula & $\mathrm{C}_{15} \mathrm{H}_{24} \mathrm{O}_{2}$ \\
\hline Formula weight & 236.34 \\
\hline Temperature/K & 100 \\
\hline Crystal system & orthorhombic \\
\hline Space group & $\mathrm{P} 2{ }_{1}{ }_{1} 2_{1}$ \\
\hline $\mathrm{a} / \AA$ & $9.7164(3)$ \\
\hline $\mathrm{b} / \AA \AA$ & $12.4263(5)$ \\
\hline $\mathrm{c} / \AA$ & $22.9456(9)$ \\
\hline$\alpha /{ }^{\circ}$ & 90 \\
\hline$\beta /{ }^{\circ}$ & 90 \\
\hline$\gamma /{ }^{\circ}$ & 90 \\
\hline Volume $/ \AA^{3}$ & $2770.43(18)$ \\
\hline Z & 8 \\
\hline$\rho_{\text {calc }} / \mathrm{cm}^{3}$ & 1.133 \\
\hline$\mu / \mathrm{mm}^{-1}$ & 0.073 \\
\hline $\mathrm{F}(000)$ & 1040.0 \\
\hline Crystal size $/ \mathrm{mm}^{3}$ & $0.343 \times 0.306 \times 0.186$ \\
\hline Radiation & $\operatorname{MoK} \alpha(\lambda=0.71073)$ \\
\hline \multicolumn{2}{|c|}{$2 \Theta$ range for data collection $/{ }^{\circ} 5.322$ to 56.754} \\
\hline Index ranges & $-12 \leq \mathrm{h} \leq 12,-16 \leq \mathrm{k} \leq 16,-30 \leq 1 \leq 30$ \\
\hline Reflections collected & 64113 \\
\hline Independent reflections & $6908\left[R_{\text {int }}=0.0538, R_{\text {sigma }}=0.0264\right]$ \\
\hline Data/restraints/parameters & $6908 / 90 / 359$ \\
\hline Goodness-of-fit on $\mathrm{F}^{2}$ & 1.049 \\
\hline Final R indexes $[\mathrm{I}>=2 \sigma(\mathrm{I})]$ & $\mathrm{R}_{1}=0.0391, \mathrm{wR}_{2}=0.0994$ \\
\hline Final $\mathrm{R}$ indexes [all data] & $\mathrm{R}_{1}=0.0436, \mathrm{wR}_{2}=0.1031$ \\
\hline \multicolumn{2}{|c|}{ Largest diff. peak/hole / e $\AA^{-3} 0.45 /-0.26$} \\
\hline Flack parameter & $0.0(3)$ \\
\hline
\end{tabular}

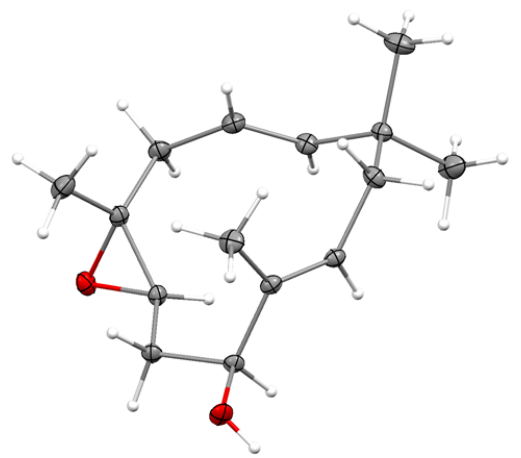


Single crystals of $\mathrm{C}_{14} \mathrm{H}_{22} \mathrm{O}_{3} 40$ were crystallized from dichloromethane/pentane solution. A suitable crystal was selected and mounted on a cryo-loop with $\left(\begin{array}{lll}2 & 1 & 0\end{array}\right)$ face roughly perpendicular to the spindle axis on a Bruker D8 Venture/Photon 100 diffractometer. The crystal was kept at $99.99 \mathrm{~K}$ during data collection. Using Olex2,22 the structure was solved with the SHELXT ${ }^{23}$ structure solution program using Intrinsic Phasing and refined with the $\mathrm{XL}^{24}$ refinement package using Least Squares minimisation.

Table S5. Hydroxyketone 40.

\begin{tabular}{|c|c|}
\hline Identification code & CCDC 2050427 \\
\hline Empirical formula & $\mathrm{C}_{14} \mathrm{H}_{22} \mathrm{O}_{3}$ \\
\hline Formula weight & 238.31 \\
\hline Temperature/K & 99.99 \\
\hline Crystal system & orthorhombic \\
\hline Space group & $\mathrm{P} 2{ }_{1} 2{ }_{1}{ }_{1}$ \\
\hline $\mathrm{a} / \AA$ & $6.3430(2)$ \\
\hline $\mathrm{b} / \AA \AA$ & $7.2691(2)$ \\
\hline $\mathrm{c} / \AA ̊$ & $27.3229(7)$ \\
\hline$\alpha /^{\circ}$ & 90 \\
\hline$\beta /{ }^{\circ}$ & 90 \\
\hline$\gamma /{ }^{\circ}$ & 90 \\
\hline Volume $/ \AA^{3}$ & $1259.80(6)$ \\
\hline $\mathrm{Z}$ & 4 \\
\hline$\rho_{\text {calc }} \mathrm{g} / \mathrm{cm}^{3}$ & 1.256 \\
\hline$\mu / \mathrm{mm}^{-1}$ & 0.694 \\
\hline $\mathrm{F}(000)$ & 520.0 \\
\hline Crystal size $/ \mathrm{mm}^{3}$ & $0.466 \times 0.14 \times 0.11$ \\
\hline Radiation & $\mathrm{CuK} \alpha(\lambda=1.54178)$ \\
\hline \multicolumn{2}{|c|}{$2 \Theta$ range for data collection $/{ }^{\circ} 6.47$ to 136.75} \\
\hline Index ranges & $-7 \leq \mathrm{h} \leq 6,-8 \leq \mathrm{k} \leq 8,-32 \leq 1 \leq 32$ \\
\hline Reflections collected & 14451 \\
\hline Independent reflections & $2317\left[\mathrm{R}_{\text {int }}=0.0331, \mathrm{R}_{\text {sigma }}=0.0203\right.$ \\
\hline Data/restraints/parameters & $2317 / 0 / 160$ \\
\hline Goodness-of-fit on $\mathrm{F}^{2}$ & 1.124 \\
\hline Final $\mathrm{R}$ indexes $[\mathrm{I}>=2 \sigma(\mathrm{I})]$ & $\mathrm{R}_{1}=0.0300, \mathrm{wR}_{2}=0.0771$ \\
\hline Final R indexes [all data] & $\mathrm{R}_{1}=0.0306, \mathrm{wR}_{2}=0.0776$ \\
\hline \multicolumn{2}{|c|}{ Largest diff. peak/hole / e $\AA^{-3} 0.23 /-0.26$} \\
\hline Flack parameter & $0.04(6)$ \\
\hline
\end{tabular}

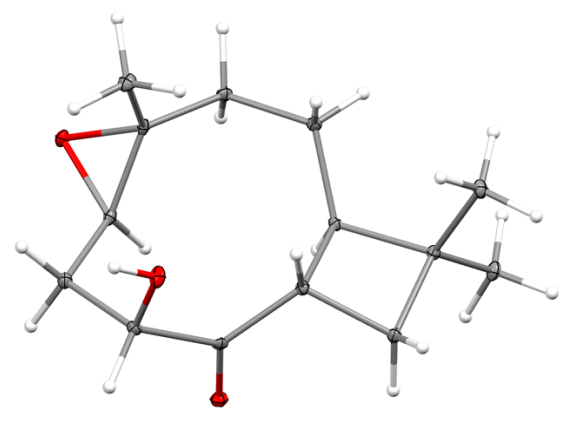


Single crystals of $\mathrm{C}_{33} \mathrm{H}_{42} \mathrm{BrNO}_{6} 48$ were crystallized from methanol. A suitable crystal was selected and mounted on a CryoLoop (Hampton Research) using Paratone-N oil (Exxon) with (1 110$)$ face roughly perpendicular to the spindle axis on a Bruker APEX-II CCD diffractometer. The crystal was kept at 99.99 $\mathrm{K}$ during data collection. Using Olex $2,{ }^{22}$ the structure was solved with the $\mathrm{XT}^{23}$ structure solution program using Intrinsic Phasing and refined with the $\mathrm{XL}^{24}$ refinement package using Least Squares minimisation. Methanol molecules in the solvate were highly ordered. Disordered solvent was modeled using OLEX Bypass procedure. A solvent mask was calculated and 204 electrons were found in a volume of $24 \AA^{3}$ in 1 void per unit cell. This is consistent with the presence of $2.5 \mathrm{H}_{2} \mathrm{O}$ per moiety which accounts for 200 electrons per unit cell.

Table S6. Epolone B carbamate 48.

\begin{tabular}{|c|c|}
\hline Identification code & CCDC 2050428 \\
\hline Empirical formula & $\mathrm{C}_{32} \mathrm{H}_{39} \mathrm{BrNO}_{6} \cdot \mathrm{CH}_{3} \mathrm{OH} \cdot 2.5 \mathrm{H}_{2} \mathrm{O}$ \\
\hline Formula weight & 628.58 \\
\hline Temperature/K & 99.99 \\
\hline Crystal system & orthorhombic \\
\hline Space group & $\mathrm{P} 2{ }_{1}{ }_{1} 2_{1}$ \\
\hline $\mathrm{a} / \AA$ & $13.6047(3)$ \\
\hline $\mathrm{b} / \AA$ & $17.4760(4)$ \\
\hline $\mathrm{c} / \AA$ & $28.4885(8)$ \\
\hline$\alpha /{ }^{\circ}$ & 90 \\
\hline$\beta /{ }^{\circ}$ & 90 \\
\hline$\gamma /{ }^{\circ}$ & 90 \\
\hline Volume $/ \AA^{3}$ & $6773.3(3)$ \\
\hline Z & 8 \\
\hline$\rho_{\text {calc }} \mathrm{g} / \mathrm{cm}^{3}$ & 1.233 \\
\hline$\mu / \mathrm{mm}^{-1}$ & 1.254 \\
\hline $\mathrm{F}(000)$ & 2640.0 \\
\hline Crystal size $/ \mathrm{mm}^{3}$ & $0.622 \times 0.184 \times 0.09$ \\
\hline Radiation & $\operatorname{MoK} \alpha(\lambda=0.71073)$ \\
\hline \multicolumn{2}{|c|}{$2 \Theta$ range for data collection $/{ }^{\circ} 4.054$ to 50.894} \\
\hline Index ranges & $-16 \leq \mathrm{h} \leq 16,-19 \leq \mathrm{k} \leq 21,-34 \leq 1 \leq 34$ \\
\hline Reflections collected & 97186 \\
\hline Independent reflections & $12478\left[\mathrm{R}_{\text {int }}=0.0494, \mathrm{R}_{\text {sigma }}=0.0264\right]$ \\
\hline Data/restraints/parameters & $12478 / 1540 / 1069$ \\
\hline Goodness-of-fit on $\mathrm{F}^{2}$ & 1.052 \\
\hline Final $R$ indexes $[\mathrm{I}>=2 \sigma(\mathrm{I})]$ & $\mathrm{R}_{1}=0.0399, \mathrm{wR}_{2}=0.0960$ \\
\hline Final $\mathrm{R}$ indexes [all data] & $\mathrm{R}_{1}=0.0458, \mathrm{wR}_{2}=0.0992$ \\
\hline \multicolumn{2}{|c|}{ Largest diff. peak/hole / e $\AA^{-3} 0.26 /-0.38$} \\
\hline Flack parameter & $0.0009(19)$ \\
\hline
\end{tabular}

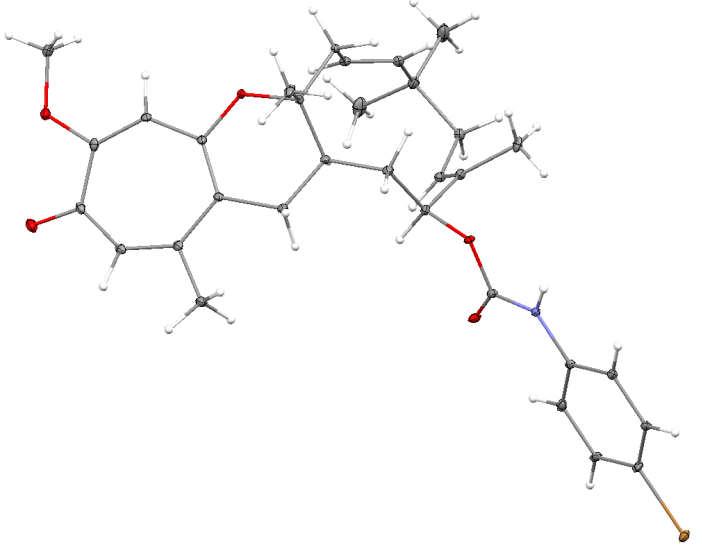


Single crystals of $\mathrm{C}_{35} \mathrm{H}_{43} \mathrm{NO}_{7} 1$ were crystallized from 100:1 acetonitrile-water by cooling down a hot solution. ${ }^{25}$ A suitable crystal was selected and mounted on a cryo-loop uing Krytox oil with (1 -2 1) face roughly perpendicular to the spindle axis. on a Bruker D8 Venture/Photon II diffractometer. The crystal was kept at $250.0 \mathrm{~K}$ during data collection. Using Olex $2^{22}$, the structure was solved with the SHELXT ${ }^{23}$ structure solution program using Intrinsic Phasing and refined with the $\mathrm{XL}^{24}$ refinement package using Least Squares minimisation.

Highly ordered acetonitrile molecules were found in the unit cell, they required no special treatment during refinement. High residual electron density was located in solvent accessible voids (picture). The refinement of atomic positions was not possible. The diffuse contributions to structure factors were removed by SQUEEZE/PLATON program. 535 electrons were found to occupy a volume of $2184 \AA^{3}$. This was in a good agreement with 54 molecules of water per unit cell, or 9 molecules of water per asymmetric unit.

Absolute stereochemistry was assigned based on Bayesian statistics of Bijvoet pair intensities (vide infra). P2 refers to a two-sided Student test $p$-value, assuming the crystal is enantiopure. P3 refers to a three-sided Student test $p$-value, with no assumption about crystal enantiopurity. True and false refers to correct and incorrect absolute stereochemistry, respectively.

Table S7. Pycnidione 1.

\begin{tabular}{|c|c|c|}
\hline Identification code & CCDC 2050425 (This work) & CCDC 1174859 (Ref. 25) \\
\hline Empirical formula & $\mathrm{C}_{33} \mathrm{H}_{40} \mathrm{O}_{7} \cdot \mathrm{CH}_{3} \mathrm{CN} \cdot 9 \mathrm{H}_{2} \mathrm{O}$ & $\mathrm{C}_{33} \mathrm{H}_{40} \mathrm{O}_{7} \cdot 4 \mathrm{H}_{2} \mathrm{O}$ \\
\hline Formula weight & 589.70 & \\
\hline Temperature/K & 250.0 & 295 \\
\hline Crystal system & hexagonal & hexagonal \\
\hline Space group & $P 6_{1}$ & $P 65$ \\
\hline $\mathrm{a} / \AA$ & $22.0172(15)$ & $22.023(8)$ \\
\hline $\mathrm{b} / \AA$ & $22.0172(15)$ & $22.023(8)$ \\
\hline $\mathrm{c} / \AA \AA$ & $15.5019(11)$ & $15.638(7)$ \\
\hline$\alpha /{ }^{\circ}$ & 90 & 90 \\
\hline$\beta /{ }^{\circ}$ & 90 & 90 \\
\hline$\gamma /{ }^{\circ}$ & 120 & 120 \\
\hline Volume $/ \AA^{3}$ & $6507.9(10)$ & 6568.48 \\
\hline $\mathrm{Z}$ & 6 & 6 \\
\hline$\rho_{\text {calc }} g / \mathrm{cm}^{3}$ & 0.903 & 0.887 \\
\hline$\mu / \mathrm{mm}^{-1}$ & 0.505 & \\
\hline $\mathrm{F}(000)$ & 1896.0 & \\
\hline Crystal size $/ \mathrm{mm}^{3}$ & $0.435 \times 0.255 \times 0.212$ & \\
\hline Radiation & $\operatorname{CuK} \alpha(\lambda=1.54178)$ & $\mathrm{CuK} \alpha(\lambda=1.5418)$ \\
\hline \multicolumn{3}{|c|}{$2 \Theta$ range for data collection $/{ }^{\circ} 4.634$ to 137.172} \\
\hline Index ranges & $-26 \leq \mathrm{h} \leq 26,-25 \leq \mathrm{k} \leq 26,-18 \leq 1 \leq 1$ & \\
\hline Reflections collected & 58262 & \\
\hline Independent reflections & $7972\left[R_{\text {int }}=0.0422, R_{\text {sigma }}=0.0252\right]$ & 1686 \\
\hline Data/restraints/parameters & $7972 / 1 / 404$ & \\
\hline Goodness-of-fit on $\mathrm{F}^{2}$ & 1.081 & \\
\hline Final $\mathrm{R}$ indexes $[\mathrm{I}>=2 \sigma(\mathrm{I})]$ & $\mathrm{R}_{1}=0.0294, \mathrm{wR}_{2}=0.0827$ & \\
\hline Final $\mathrm{R}$ indexes [all data] & $\mathrm{R}_{1}=0.0307, \mathrm{wR}_{2}=0.0837$ & \\
\hline \multicolumn{3}{|c|}{ Largest diff. peak/hole / e $\AA^{-3} 0.10 /-0.09$} \\
\hline Flack $x$ parameter & $0.18(7)$ & \\
\hline Hooft $y$ parameter & $0.17(6)$ & \\
\hline Parsons $z$ parameter & $0.17(5)$ & \\
\hline Bayesian P2(True) & 1.000 & \\
\hline Bayesian P3 (True) & 1.000 & \\
\hline
\end{tabular}


Bayesian P3 (Racemic Twin) $4 \times 10^{-5}$

Bayesian P3 (False) $2 \times 10^{-36}$

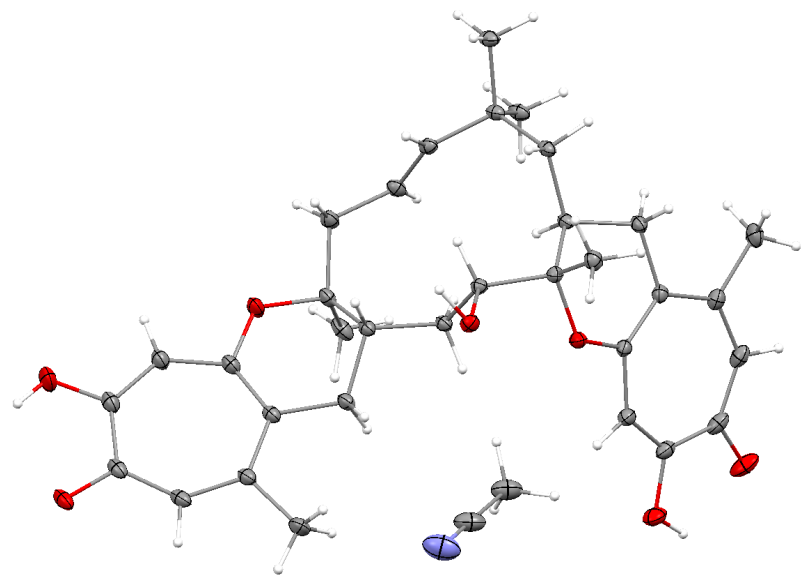




\title{
Comparisons of Natural and Synthetic Compounds
}

\section{Epolone B Comparison}

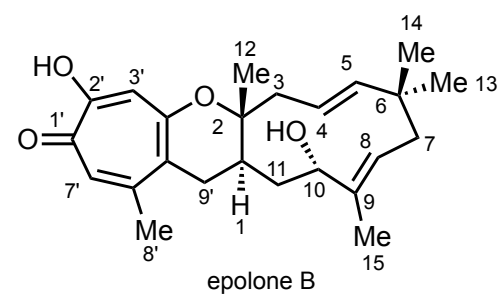

\author{
Epolone B Source \\ Natural (Houck) $)^{26}$ \\ Biosynthetic $(\mathrm{Hu})^{12}$ \\ Synthetic (Sarlah)
}
$[\alpha]_{\mathbf{D}}$ $+85.0\left(\right.$ c $\left.0.33, \mathrm{CH}_{2} \mathrm{Cl}_{2}\right)$
$+141.1\left(c 0.33, \mathrm{CHCl}_{3}\right)$
$-84.0\left(\right.$ c $\left.0.35, \mathrm{CHCl}_{3}\right)$

Table S8. ${ }^{1} \mathrm{H}$ NMR comparison of epolone B.

\begin{tabular}{|c|c|c|c|}
\hline $\begin{array}{c}\text { proton } \\
\#\end{array}$ & $\begin{array}{c}\text { Natural (Houck) } \\
500 \text { MHz, CDCl } \\
\delta^{1}{ }^{1} \mathrm{Hpm} \\
\text { (mult, } J=\text { Hz) }\end{array}$ & $\begin{array}{c}\text { Biosynthetic }(\mathrm{Hu}) \\
600 \mathrm{MHz}, \mathrm{CDCl}_{3}, \\
\delta^{1} \mathrm{H} \text { ppm } \\
(\mathrm{mult}, J=\mathrm{Hz})\end{array}$ & $\begin{array}{c}\text { Synthetic (Sarlah) } \\
500 \mathrm{MHz}, \mathrm{CDCl}_{3}, \\
\delta^{1} \mathrm{H} \text { ppm } \\
\text { (mult, } J=\mathrm{Hz})\end{array}$ \\
\hline $3^{\prime}$ & $6.97(\mathrm{~s})$ & $6.98(\mathrm{~s})$ & $6.99(\mathrm{~s})$ \\
\hline $7^{\prime}$ & $7.10(\mathrm{~s})$ & $7.10(\mathrm{~s})$ & $7.12(\mathrm{~s})$ \\
\hline $8^{\prime}$ & $2.39(\mathrm{~s}, 3 \mathrm{H})$ & $2.38(\mathrm{~s}, 3 \mathrm{H})$ & $2.40(\mathrm{~s}, 3 \mathrm{H})$ \\
\hline \multirow[t]{2}{*}{$9^{\prime}$} & $2.88(\mathrm{dd}, 17,5.5)$ & $2.89(\mathrm{dd}, 17.5,4.9)$ & $2.90(\mathrm{dd}, 17,4.0)$ \\
\hline & $2.36(\mathrm{dd}, 17,12.5)$ & $2.35(\mathrm{~m})$ & $2.36(\mathrm{~m})$ \\
\hline 1 & $1.64(\mathrm{~m})$ & $1.67(\mathrm{~m})$ & $1.68(\mathrm{~m})$ \\
\hline \multirow[t]{2}{*}{3} & $2.56(\mathrm{bd}, 14.5)$ & $2.56(\mathrm{~d}, 14.5)$ & $2.58(\mathrm{bd}, 14.8)$ \\
\hline & $2.23(\mathrm{bd}, 14.5,10)$ & $2.25(\mathrm{~m})$ & $2.25(\mathrm{~m})$ \\
\hline 4 & $5.04(\mathrm{ddd}, 15.5,10,3)$ & $5.03(\mathrm{dd}, 15.7,10.6)$ & $5.05(\mathrm{ddd}, 15.9,10.4,2.6)$ \\
\hline 5 & $5.14(\mathrm{dd}, 15.5,2)$ & $5.16(\mathrm{~m})$ & $5.19(\mathrm{~m})$ \\
\hline \multirow[t]{2}{*}{7} & $2.22(\mathrm{dd}, 12.5,6)$ & $2.21(\mathrm{~m})$ & $2.25(\mathrm{~m})$ \\
\hline & $1.77(\mathrm{dd}, 12.5,5)$ & $1.76(\mathrm{~m})$ & $1.77(\mathrm{~m})$ \\
\hline 8 & $5.20(\mathrm{dd}, 6,5)$ & $5.17(\mathrm{~m})$ & $5.19(\mathrm{~m})$ \\
\hline 10 & $3.94(\mathrm{bd}, 10)$ & $3.96(\mathrm{~d}, 9.9)$ & $3.97(\mathrm{bd}, 9.8)$ \\
\hline \multirow[t]{2}{*}{11} & $1.72(\mathrm{dd}, 14,10)$ & $1.72(\mathrm{~m})$ & $1.77(\mathrm{~m})$ \\
\hline & $1.12(\mathrm{bdd}, 14,8)$ & 1.12 (bdd, 14, 8) & $1.16(\mathrm{~m})$ \\
\hline 12 & $1.10(\mathrm{~s}, 3 \mathrm{H})$ & $1.10(\mathrm{~s}, 3 \mathrm{H})$ & $1.12(\mathrm{~s}, 3 \mathrm{H})$ \\
\hline 13 & $1.06(\mathrm{~s}, 3 \mathrm{H})$ & $1.06(\mathrm{~s}, 3 \mathrm{H})$ & $1.07(\mathrm{~s}, 3 \mathrm{H})$ \\
\hline 14 & $0.99(\mathrm{~s}, 3 \mathrm{H})$ & $0.99(\mathrm{~s}, 3 \mathrm{H})$ & $1.01(\mathrm{~s}, 3 \mathrm{H})$ \\
\hline 15 & $1.63(\mathrm{~s}, 3 \mathrm{H})$ & $1.64(\mathrm{~s}, 3 \mathrm{H})$ & $1.65(\mathrm{~s}, 3 \mathrm{H})$ \\
\hline
\end{tabular}




\section{Epolone B Comparison (cont.)}

Table S9. ${ }^{13} \mathrm{C}$ NMR comparison of epolone B.

\begin{tabular}{|c|c|c|c|}
\hline $\begin{array}{c}\text { carbon } \\
\quad \#\end{array}$ & $\begin{array}{c}\text { Natural (Houck) } \\
100 \mathrm{MHz}, \mathrm{CDCl}_{3}, \\
\delta{ }^{13} \mathrm{C} \mathrm{ppm}\end{array}$ & $\begin{array}{c}\text { Biosynthetic }(\mathrm{Hu}) \\
150 \mathrm{MHz}, \mathrm{CDCl}_{3}, \\
\delta^{13} \mathrm{Cppm}\end{array}$ & $\begin{array}{c}\text { Synthetic (Sarlah) } \\
126 \mathrm{MHz}, \mathrm{CDCl}_{3} \\
\delta^{13} \mathrm{C} \mathrm{ppm}\end{array}$ \\
\hline $1^{\prime}$ & 172.7 & 172.6 & 172.8 \\
\hline $2^{\prime}$ & 163.1 & 163.3 & 163.1 \\
\hline $3^{\prime}$ & 113.0 & 113.5 & 113.3 \\
\hline $4^{\prime}$ & 160.9 & 161.2 & 161.1 \\
\hline $5^{\prime}$ & 119.9 & 120.4 & 120.2 \\
\hline $6^{\prime}$ & 149.8 & 150.1 & 150.1 \\
\hline $\begin{array}{l}0 \\
7^{\prime}\end{array}$ & 124.5 & 124.5 & 124.6 \\
\hline $8^{\prime}$ & 38.7 & 38.7 & 38.8 \\
\hline $\begin{array}{l}8 \\
9^{\prime}\end{array}$ & 31.3 & 31.4 & 31.5 \\
\hline 9. & 33.4 & 33.5 & 33.6 \\
\hline 1 & 81.3 & 81.5 & 81.5 \\
\hline $\begin{array}{l}2 \\
3\end{array}$ & 42.6 & 42.6 & 42.7 \\
\hline 3 & 120.3 & 120.4 & 120.4 \\
\hline $\begin{array}{l}4 \\
5\end{array}$ & 142.2 & 142.3 & 142.3 \\
\hline $\begin{array}{l}5 \\
6\end{array}$ & 38.6 & 38.8 & 38.8 \\
\hline $\begin{array}{l}0 \\
7\end{array}$ & 40.6 & 40.7 & 40.8 \\
\hline 8 & 123.8 & 123.7 & 124.0 \\
\hline $\begin{array}{l}8 \\
9\end{array}$ & 138.4 & 138.7 & 138.5 \\
\hline 10 & 77.9 & 77.9 & 78.1 \\
\hline 11 & 27.2 & 27.4 & 27.5 \\
\hline 12 & 20.1 & 20.3 & 20.3 \\
\hline 13 & 30.0 & 30.2 & 30.2 \\
\hline 14 & 24.1 & 24.2 & 24.2 \\
\hline 15 & 10.6 & 10.8 & 10.8 \\
\hline
\end{tabular}




\section{Isoepolone B Comparison}

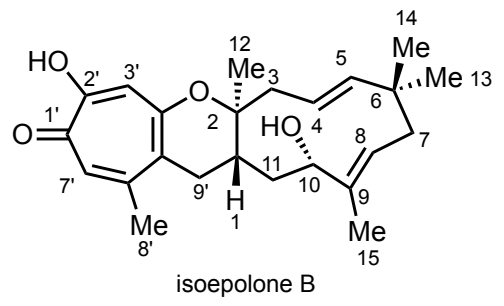

Isoepolone B

Biosynthetic $(\mathrm{Hu})^{12}$

Synthetic (Sarlah)
$[\boldsymbol{\alpha}]_{\mathbf{D}}$

$+101.3\left(c\right.$ $\left.0.08, \mathrm{CHCl}_{3}\right)$

$+65.5\left(c 1.00, \mathrm{CHCl}_{3}\right)$

Table S10. ${ }^{1} \mathrm{H}$ NMR comparison of isoepolone B.

\begin{tabular}{|c|c|c|}
\hline $\begin{array}{c}\text { proton } \\
\quad \#\end{array}$ & $\begin{array}{c}\text { Biosynthetic }(\mathrm{Hu}) \\
600 \mathrm{MHz}, \mathrm{CDCl}_{3}, \\
\delta^{1} \mathrm{H} \text { ppm } \\
(\mathrm{mult}, J=\mathrm{Hz})\end{array}$ & $\begin{array}{c}\text { Synthetic (Sarlah) } \\
500 \mathrm{MHz}, \mathrm{CDCl}_{3}, \\
\delta^{1} \mathrm{H} \mathrm{ppm} \\
(\mathrm{mult}, J=\mathrm{Hz})\end{array}$ \\
\hline $3^{\prime}$ & $7.00(\mathrm{~s})$ & $7.00(\mathrm{~s})$ \\
\hline $7^{\prime}$ & $7.11(\mathrm{~s})$ & $7.11(\mathrm{~s})$ \\
\hline $8^{\prime}$ & $2.39(\mathrm{~s}, 3 \mathrm{H})$ & $2.39(\mathrm{~s}, 3 \mathrm{H})$ \\
\hline \multirow[t]{2}{*}{$9^{\prime}$} & $2.26(\mathrm{~m})$ & $2.90(\mathrm{dd}, 17.7,11.8)$ \\
\hline & $3.22(17.8,4.7)$ & $3.23(\mathrm{dd}, 17.6,5.3)$ \\
\hline 1 & $2.01(\mathrm{~m})$ & $2.02(\mathrm{dt}, 12.0,6.1)$ \\
\hline \multirow[t]{2}{*}{3} & $2.58(\mathrm{~d}, 14.5)$ & $2.58(\mathrm{dt}, 14.8,2.1)$ \\
\hline & $2.18(\mathrm{~m})$ & $2.23-2.16(\mathrm{~m})$ \\
\hline 4 & $5.07(\mathrm{ddd}, 15.7,9.8,1.9)$ & $5.05(\mathrm{ddd}, 15.9,9.8,2.4)$ \\
\hline 5 & $5.16(\mathrm{~d}, 15.9)$ & $5.16(\mathrm{dd}, 15.9,1.8)$ \\
\hline \multirow[t]{2}{*}{7} & $1.84(\mathrm{dd}, 12.5,4.7)$ & $1.85(\mathrm{dd}, 12.6,5.1)$ \\
\hline & $2.22(\mathrm{~m})$ & $2.23-2.16(\mathrm{~m})$ \\
\hline 8 & $5.41(\mathrm{dd}, 11.1,4.7)$ & $5.42(\mathrm{dd}, 11.7,5.1)$ \\
\hline 10 & $4.36(\mathrm{~d}, 6.4)$ & $4.36(\mathrm{~d}, 6.6)$ \\
\hline \multirow[t]{2}{*}{11} & $1.56(\mathrm{~m})$ & $1.56-1.52(\mathrm{~m})$ \\
\hline & $1.50(\mathrm{~m})$ & $1.50(\mathrm{~d}, 14.2)$ \\
\hline 12 & $1.11(\mathrm{~s}, 3 \mathrm{H})$ & $1.12(\mathrm{~s}, 3 \mathrm{H})$ \\
\hline 13 & $1.09(\mathrm{~s}, 3 \mathrm{H})$ & $1.09(\mathrm{~s}, 3 \mathrm{H})$ \\
\hline 14 & $1.04(\mathrm{~s}, 3 \mathrm{H})$ & $1.04(\mathrm{~s}, 3 \mathrm{H})$ \\
\hline 15 & $1.55(\mathrm{~s}, 3 \mathrm{H})$ & $1.55(\mathrm{~s}, 3 \mathrm{H})$ \\
\hline
\end{tabular}




\section{Isoepolone B Comparison (cont.)}

Table S11. ${ }^{13} \mathrm{C}$ NMR comparison of isoepolone B.

\begin{tabular}{|c|c|c|}
\hline $\begin{array}{c}\text { carbon } \\
\#\end{array}$ & $\begin{array}{c}\text { Biosynthetic }(\mathrm{Hu}) \\
150 \mathrm{MHz}, \mathrm{CDCl}_{3}, \\
\delta{ }^{13} \mathrm{C} \mathrm{ppm}\end{array}$ & $\begin{array}{c}\text { Synthetic (Sarlah) } \\
126 \mathrm{MHz}, \mathrm{CDCl}_{3} \\
\delta^{13} \mathrm{C} \mathrm{ppm}\end{array}$ \\
\hline $1^{\prime}$ & 172.4 & 172.4 \\
\hline $2^{\prime}$ & 163.4 & 163.4 \\
\hline $3^{\prime}$ & 113.5 & 113.5 \\
\hline $4^{\prime}$ & 161.2 & 161.2 \\
\hline $5^{\prime}$ & 122.0 & 120.0 \\
\hline $6^{\prime}$ & 150.1 & 150.1 \\
\hline $7^{\prime}$ & 124.3 & 124.4 \\
\hline $8^{\prime}$ & 27.4 & 27.4 \\
\hline $9^{\prime}$ & 34.2 & 34.2 \\
\hline 1 & 29.4 & 29.3 \\
\hline 2 & 82.1 & 81.1 \\
\hline 3 & 42.8 & 42.8 \\
\hline 4 & 120.8 & 120.9 \\
\hline 5 & 141.9 & 142.0 \\
\hline 6 & 38.9 & 38.9 \\
\hline 7 & 40.9 & 40.9 \\
\hline 8 & 120.8 & 120.8 \\
\hline 9 & 134.9 & 139.4 \\
\hline 10 & 73.7 & 73.8 \\
\hline 11 & 34.7 & 34.7 \\
\hline 12 & 20.6 & 20.6 \\
\hline 13 & 30.0 & 30.0 \\
\hline 14 & 24.3 & 24.3 \\
\hline 15 & 16.5 & 16.5 \\
\hline
\end{tabular}




\section{Dehydroxypycnidione Comparison}
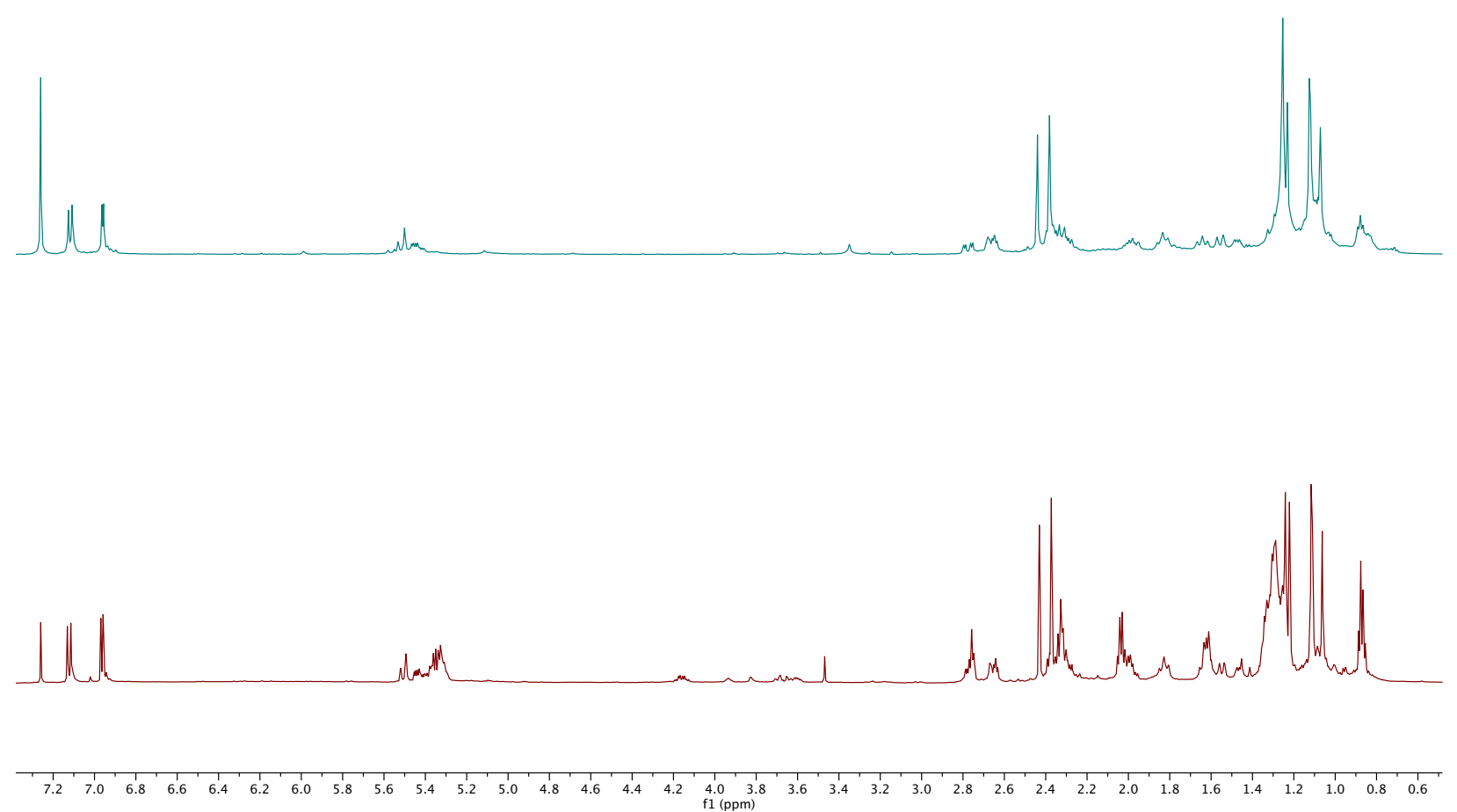

Figure S23. ${ }^{1} \mathrm{H}$ NMR comparison of our synthetic dehydroxypycnidione (top) with natural material reported by the isolation group ${ }^{27}$ (bottom). 


\section{Spectroscopic Data}

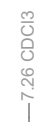<smiles>[X]OC(Cl)=CCOCOC1=CC(=O)CC1</smiles>

22-Z

( $\left.{ }^{1} \mathrm{H} \mathrm{NMR}, \mathrm{CDCl}_{3}, 500 \mathrm{MHz}\right)$
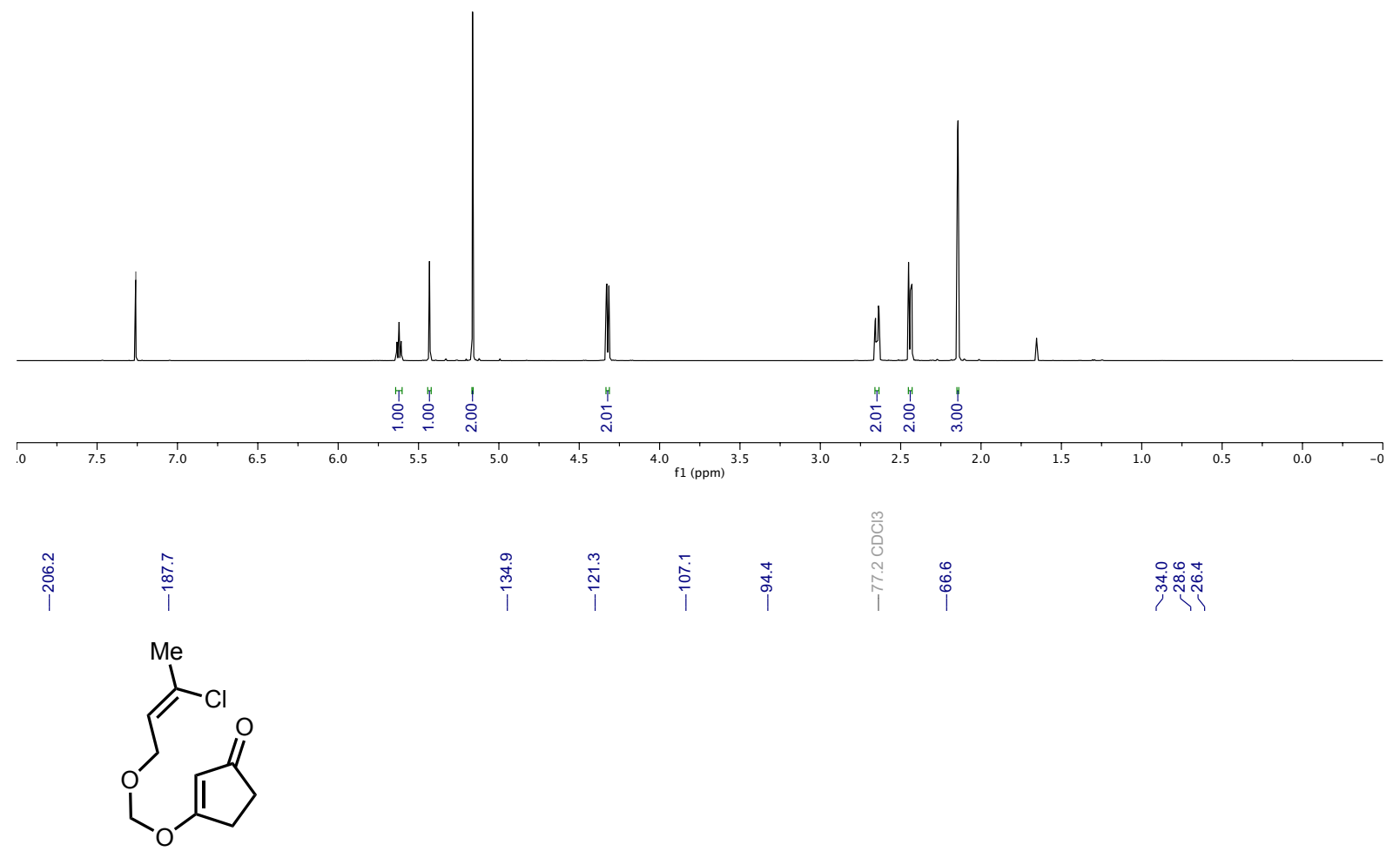

22-Z

$\left({ }^{13} \mathrm{C} N M R, \mathrm{CDCl}_{3}, 126 \mathrm{MHz}\right)$

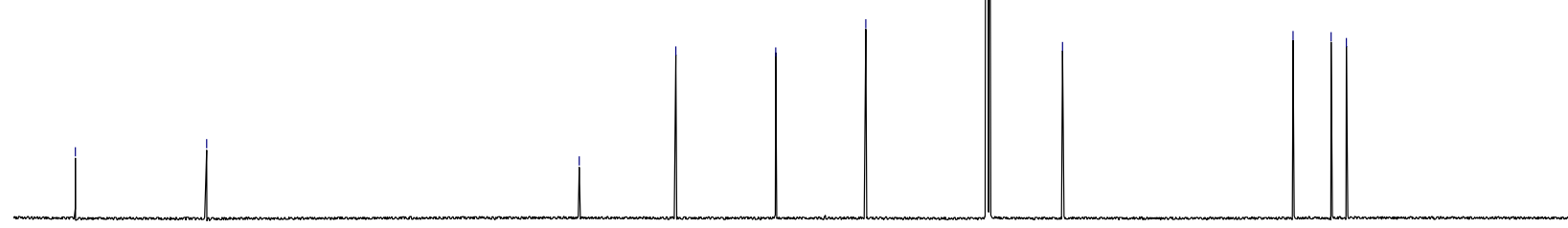

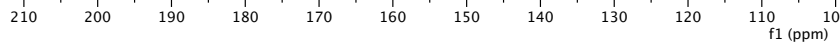




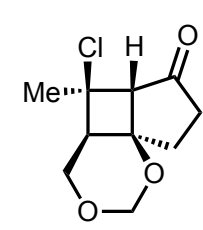

33

( ${ }^{1} \mathrm{H}$ NMR, $\mathrm{CD}_{2} \mathrm{Cl}_{2}, 500 \mathrm{MHz}$ )

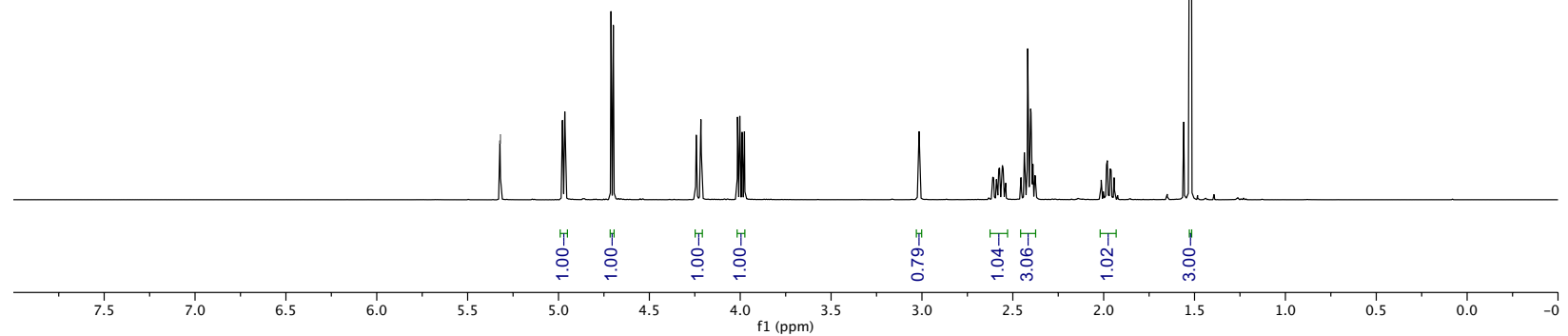

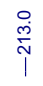
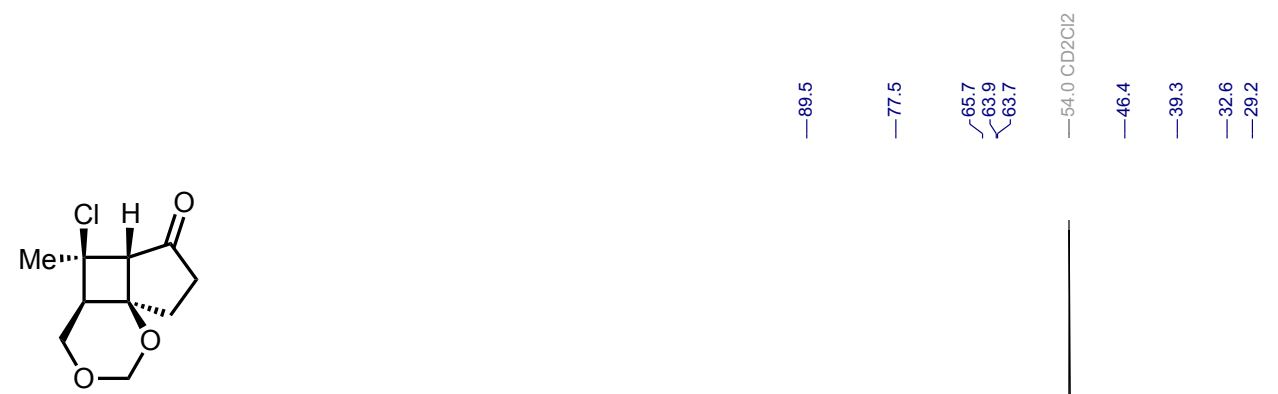

33

$\left({ }^{13} \mathrm{C} \mathrm{NMR}, \mathrm{CD}_{2} \mathrm{Cl}_{2}, 126 \mathrm{MHz}\right)$
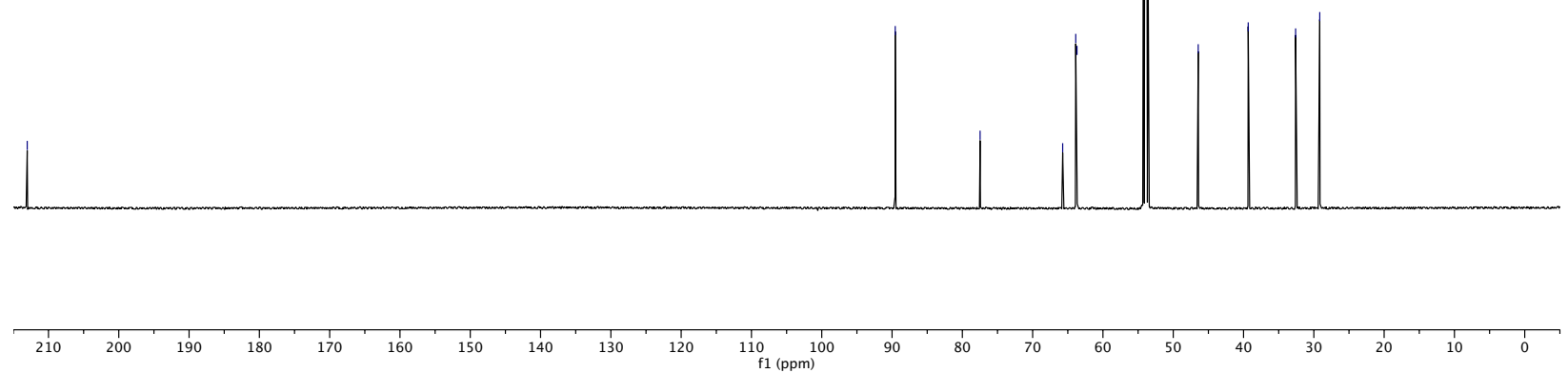


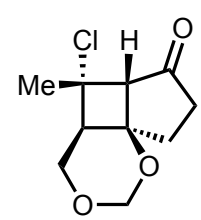

33'

$\left({ }^{1} \mathrm{H} \mathrm{NMR}, \mathrm{CD}_{2} \mathrm{Cl}_{2}, 500 \mathrm{MHz}\right)$

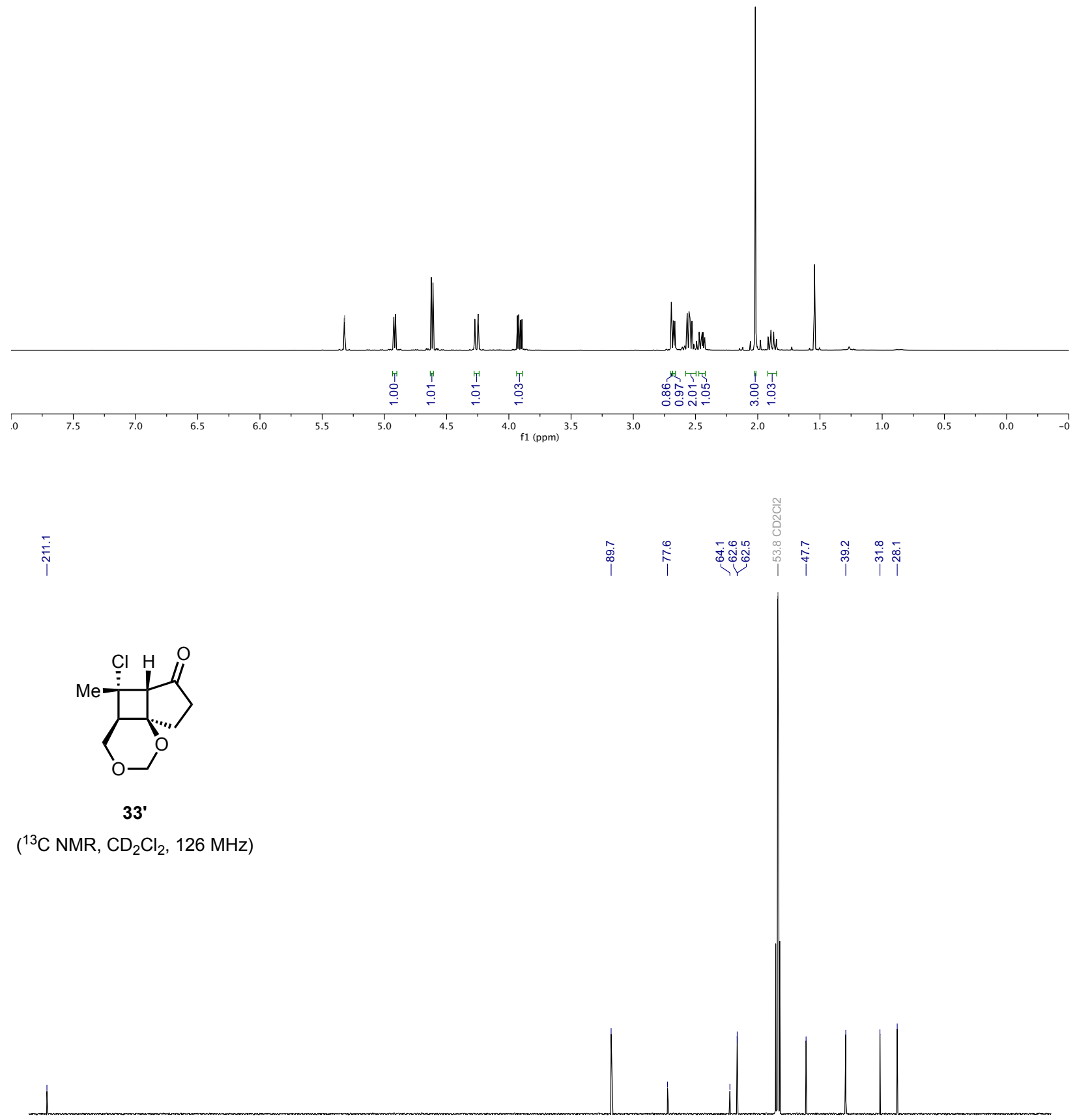

$\begin{array}{lllllllllll}210 & 200 & 190 & 180 & 170 & 160 & 150 & 140 & 130 & 120 & \underset{\mathrm{f} 1(\mathrm{ppm})}{100}\end{array}$ 


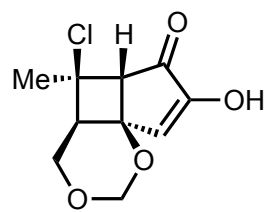

21

$\left({ }^{1} \mathrm{H} N M R, \mathrm{CDCl}_{3}, 500 \mathrm{MHz}\right)$
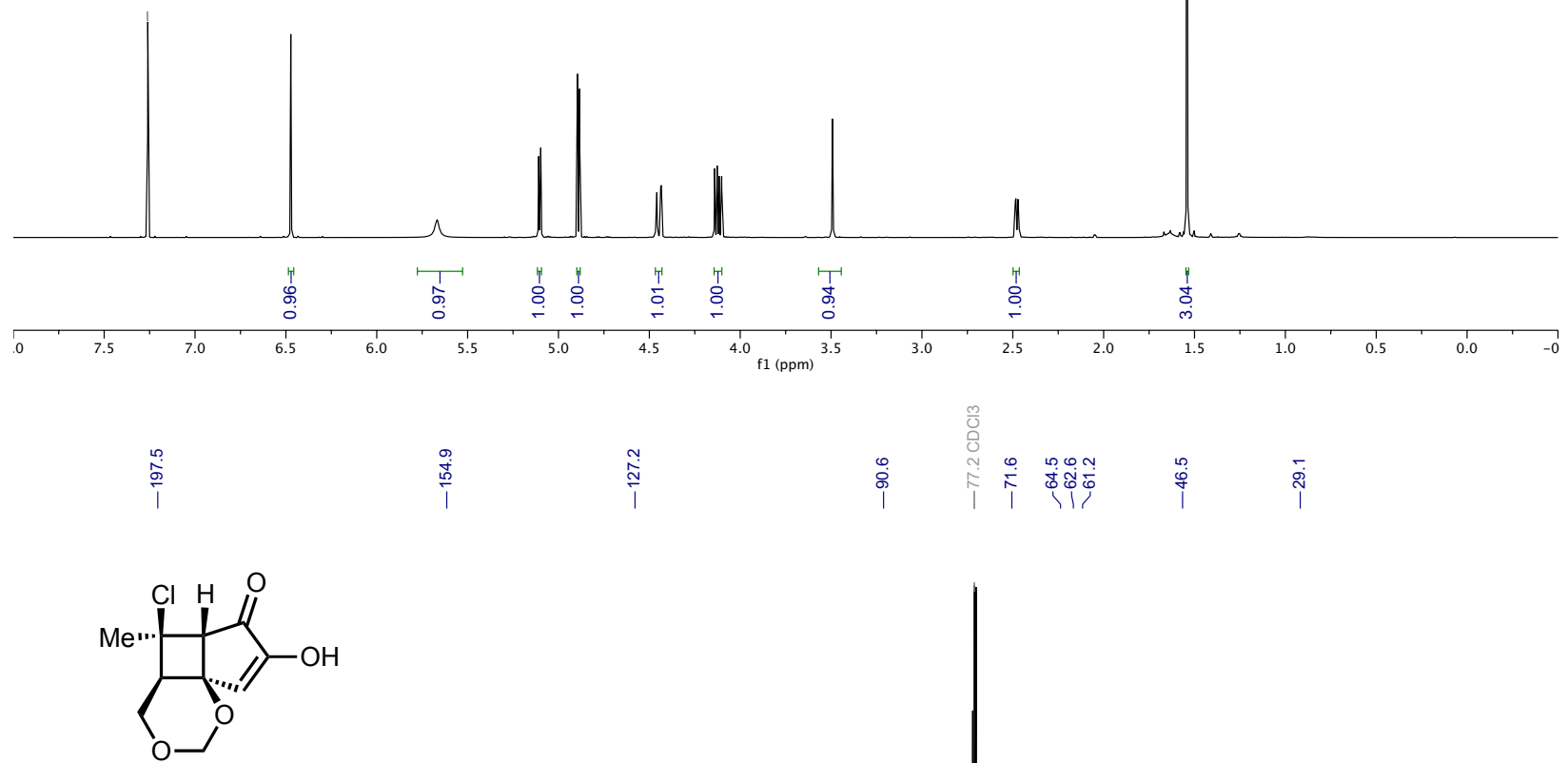

21

$\left({ }^{13} \mathrm{C} \mathrm{NMR}, \mathrm{CDCl}_{3}, 126 \mathrm{MHz}\right)$

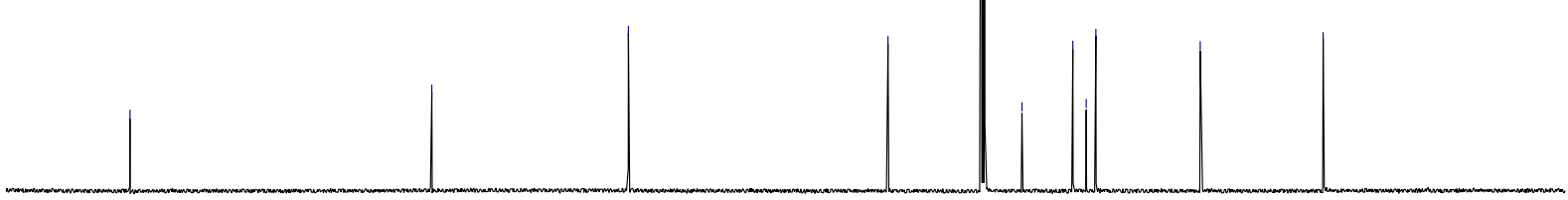




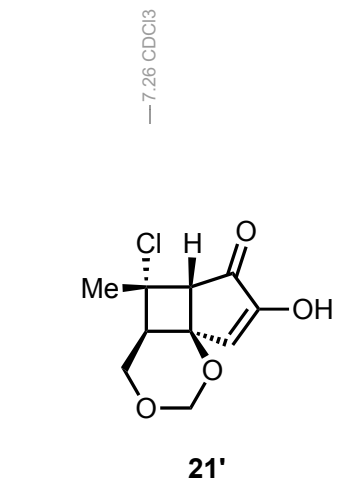

( ${ }^{1} \mathrm{H}$ NMR, $\left.\mathrm{CDCl}_{3}, 500 \mathrm{MHz}\right)$
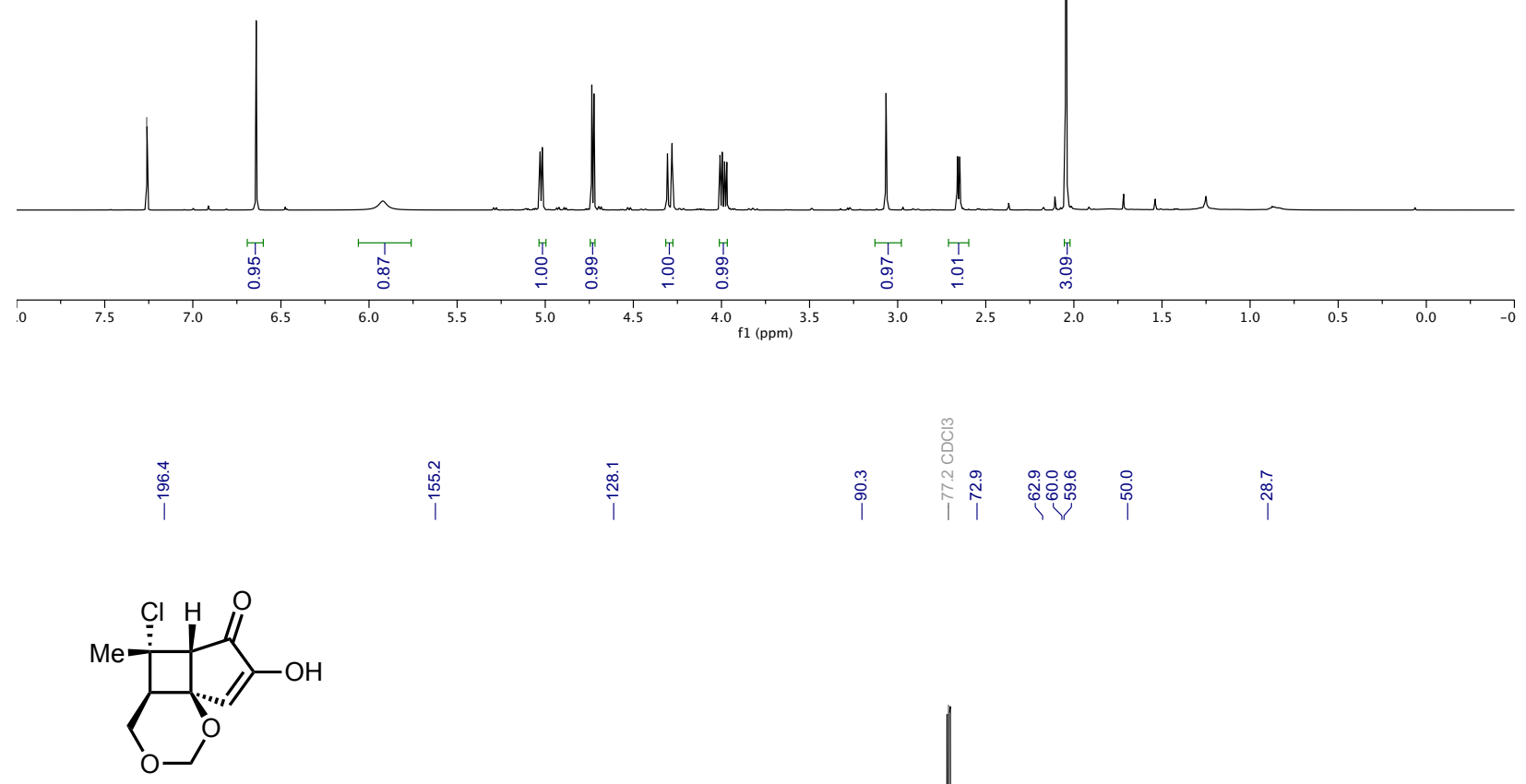

$21^{\prime}$

$\left({ }^{13} \mathrm{C} \mathrm{NMR}, \mathrm{CDCl}_{3}, 126 \mathrm{MHz}\right)$
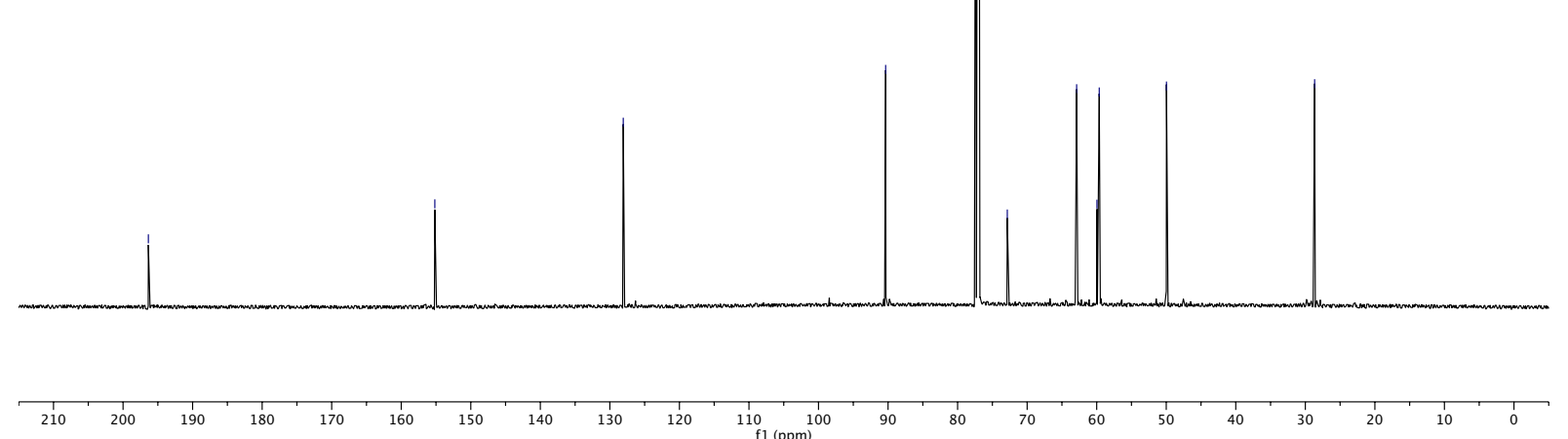


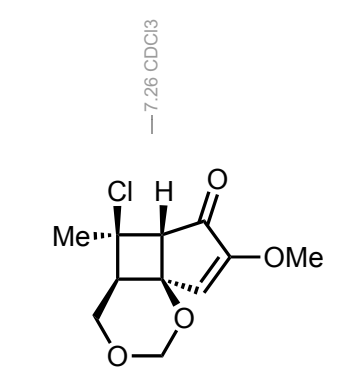

35

( ${ }^{1} \mathrm{H}$ NMR, $\mathrm{CDCl}_{3}, 500 \mathrm{MHz}$ )

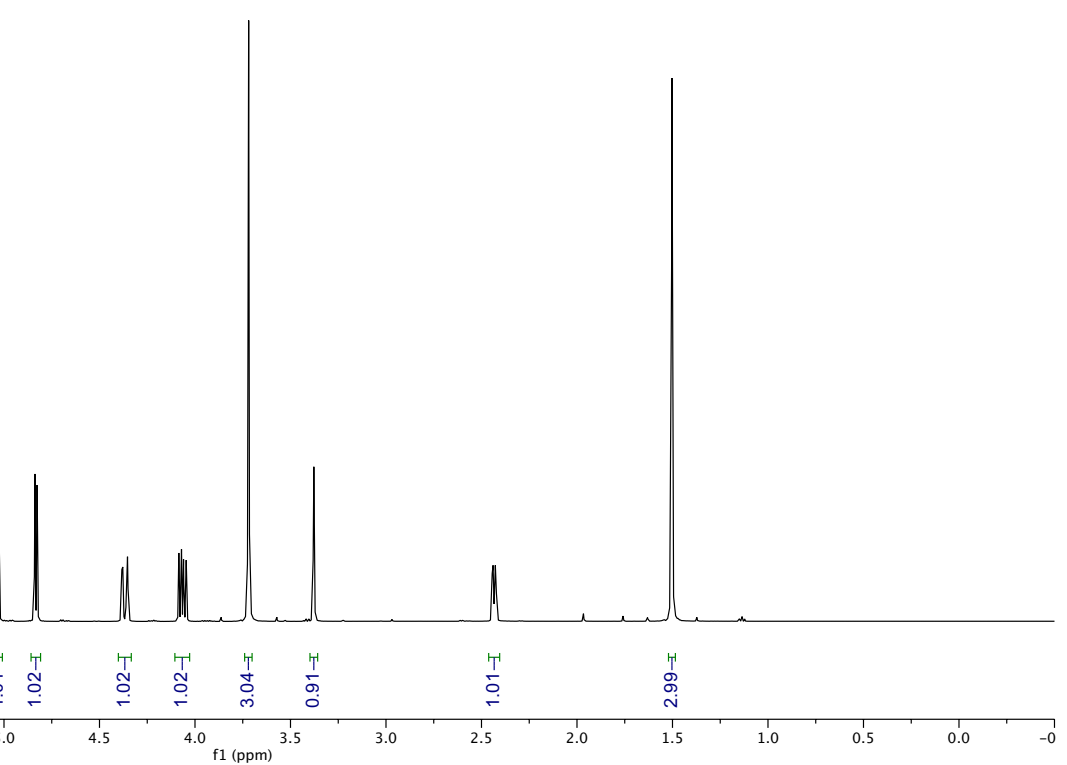

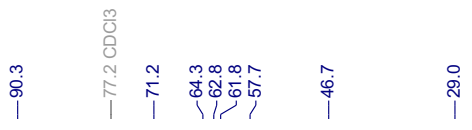

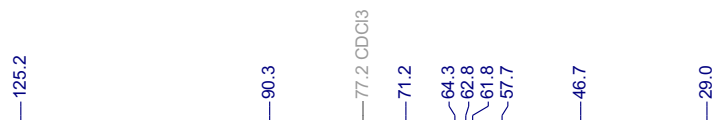

35

$\left({ }^{13} \mathrm{C} \mathrm{NMR}, \mathrm{CDCl}_{3}, 126 \mathrm{MHz}\right)$
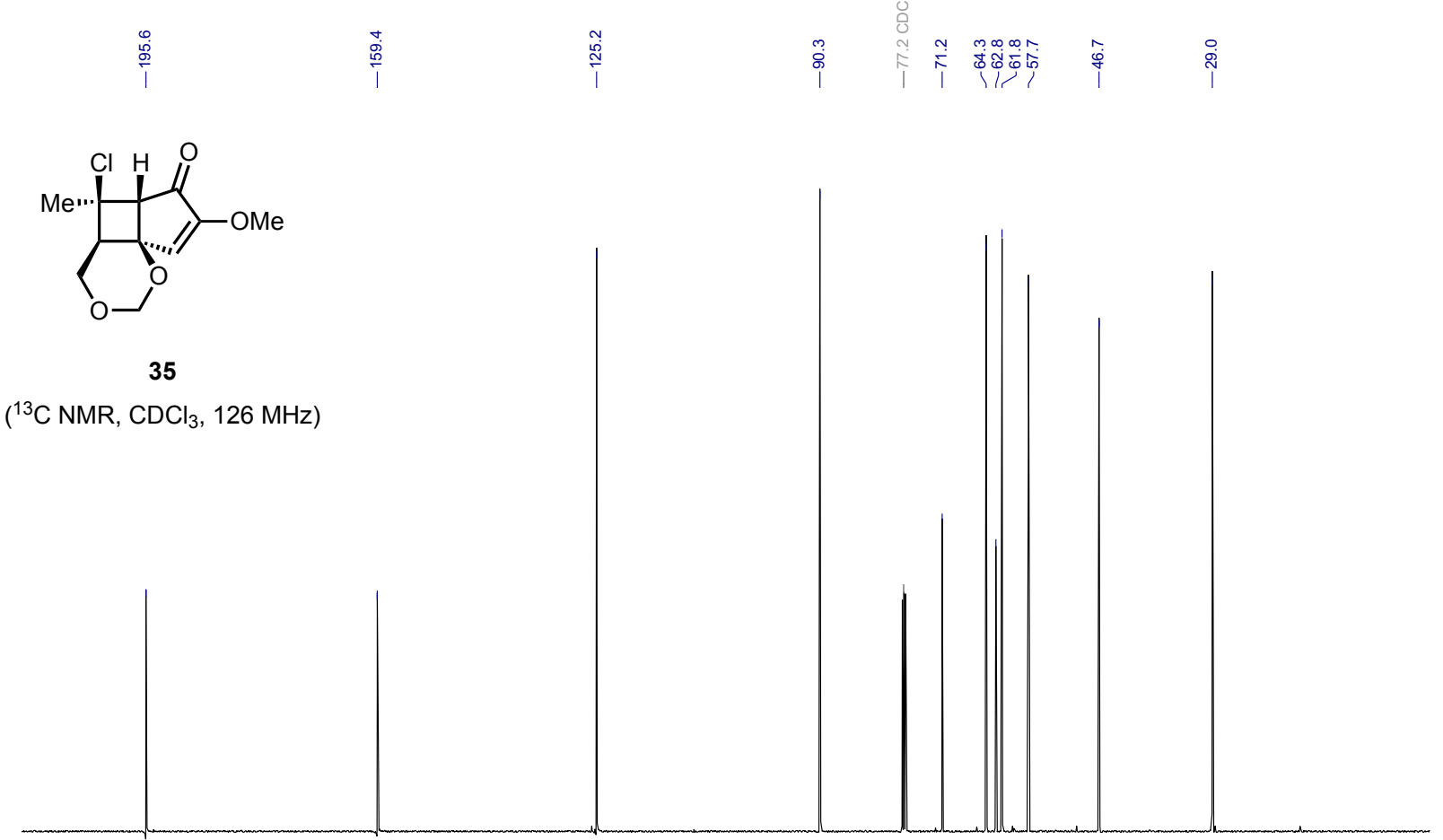

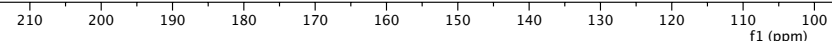




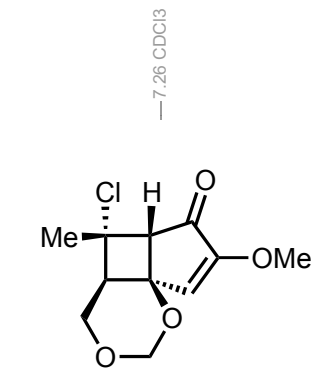

35'

$\left({ }^{1} \mathrm{H} \mathrm{NMR}, \mathrm{CDCl}_{3}, 500 \mathrm{MHz}\right.$ )
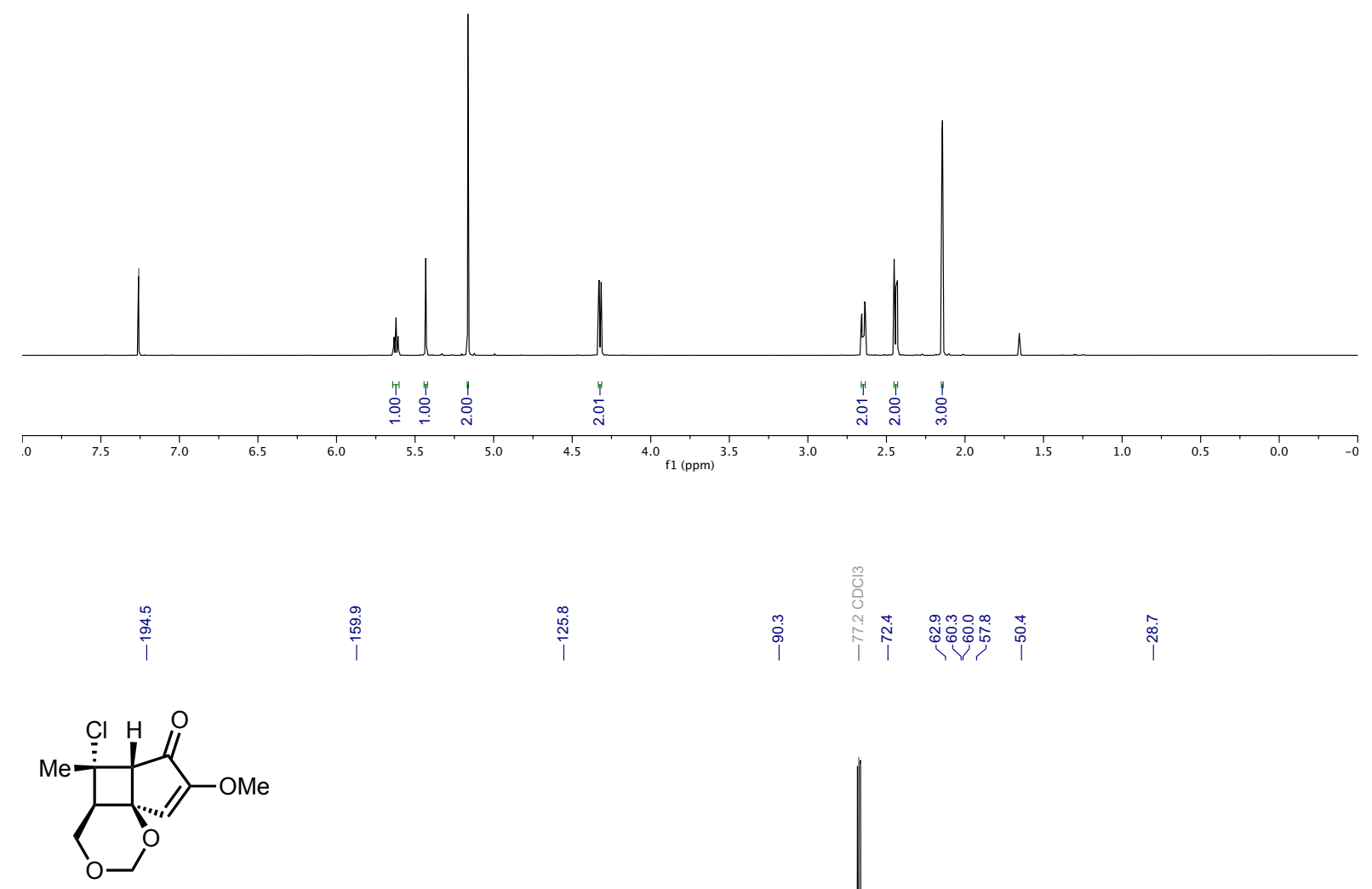

$35^{\prime}$

$\left({ }^{13} \mathrm{C} \mathrm{NMR}, \mathrm{CDCl}_{3}, 126 \mathrm{MHz}\right)$
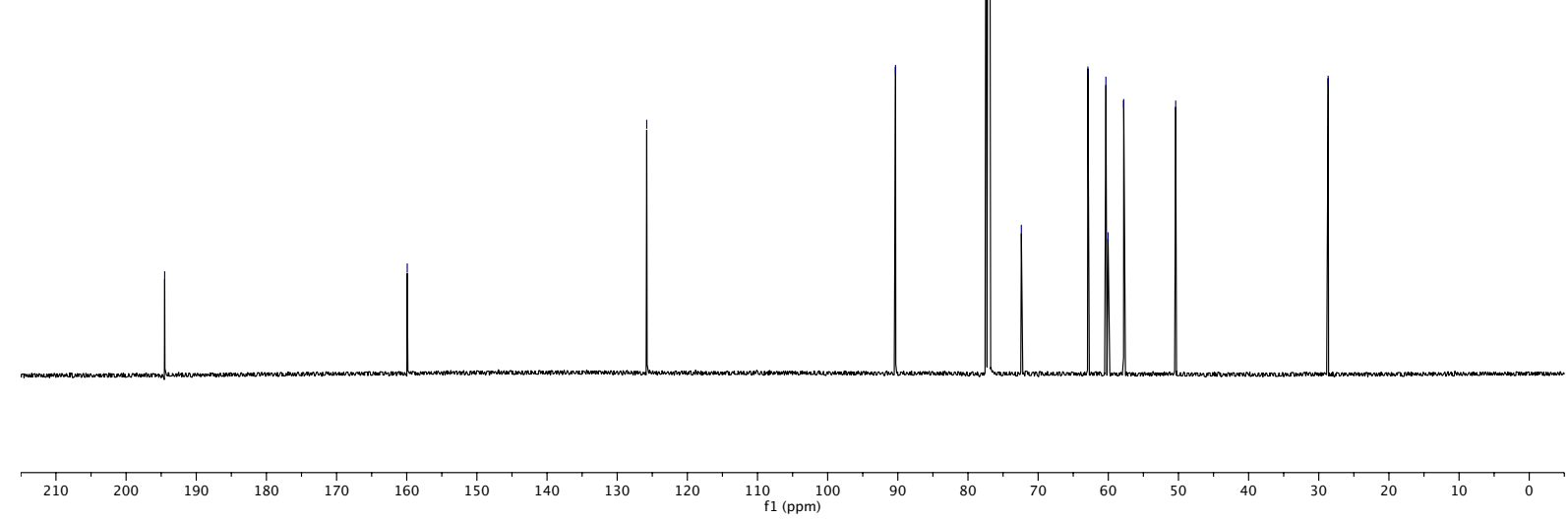


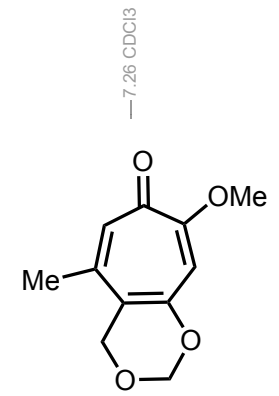

20

$\left({ }^{1} \mathrm{H} \mathrm{NMR}, \mathrm{CDCl}_{3}, 500 \mathrm{MHz}\right)$
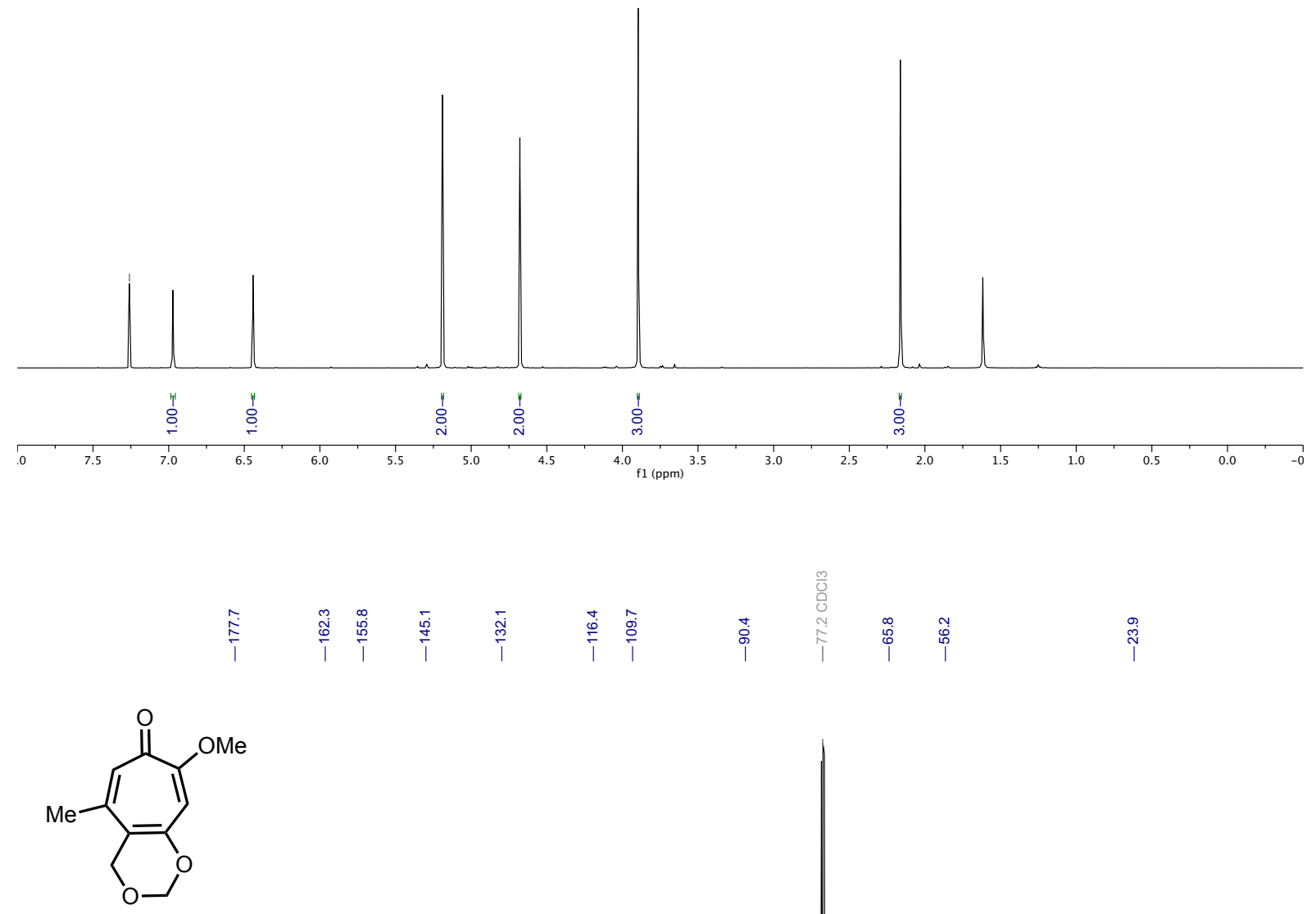

20

$\left({ }^{13} \mathrm{C} \mathrm{NMR}, \mathrm{CDCl}_{3}, 126 \mathrm{MHz}\right)$
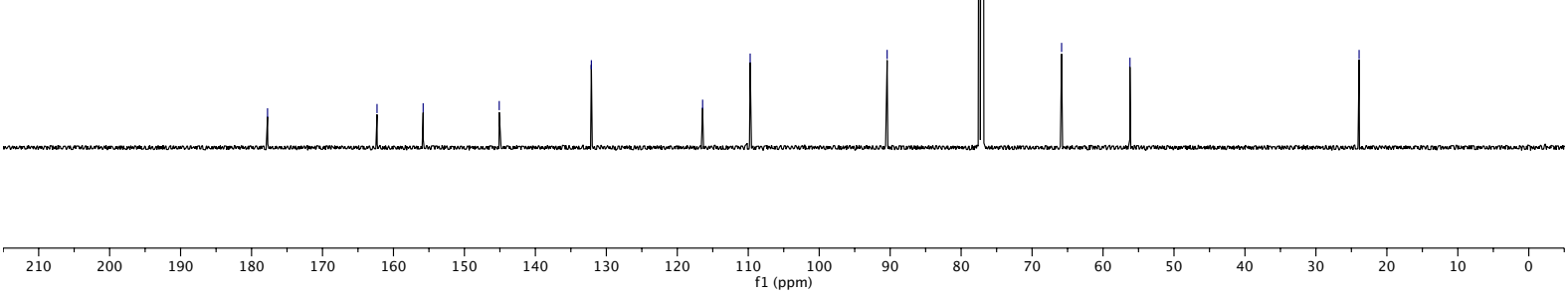


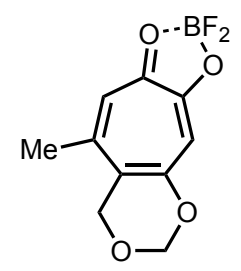

19

$\left({ }^{1} \mathrm{H}\right.$ NMR, $\left.\left(\mathrm{CD}_{3}\right)_{2} \mathrm{CO}, 500 \mathrm{MHz}\right)$
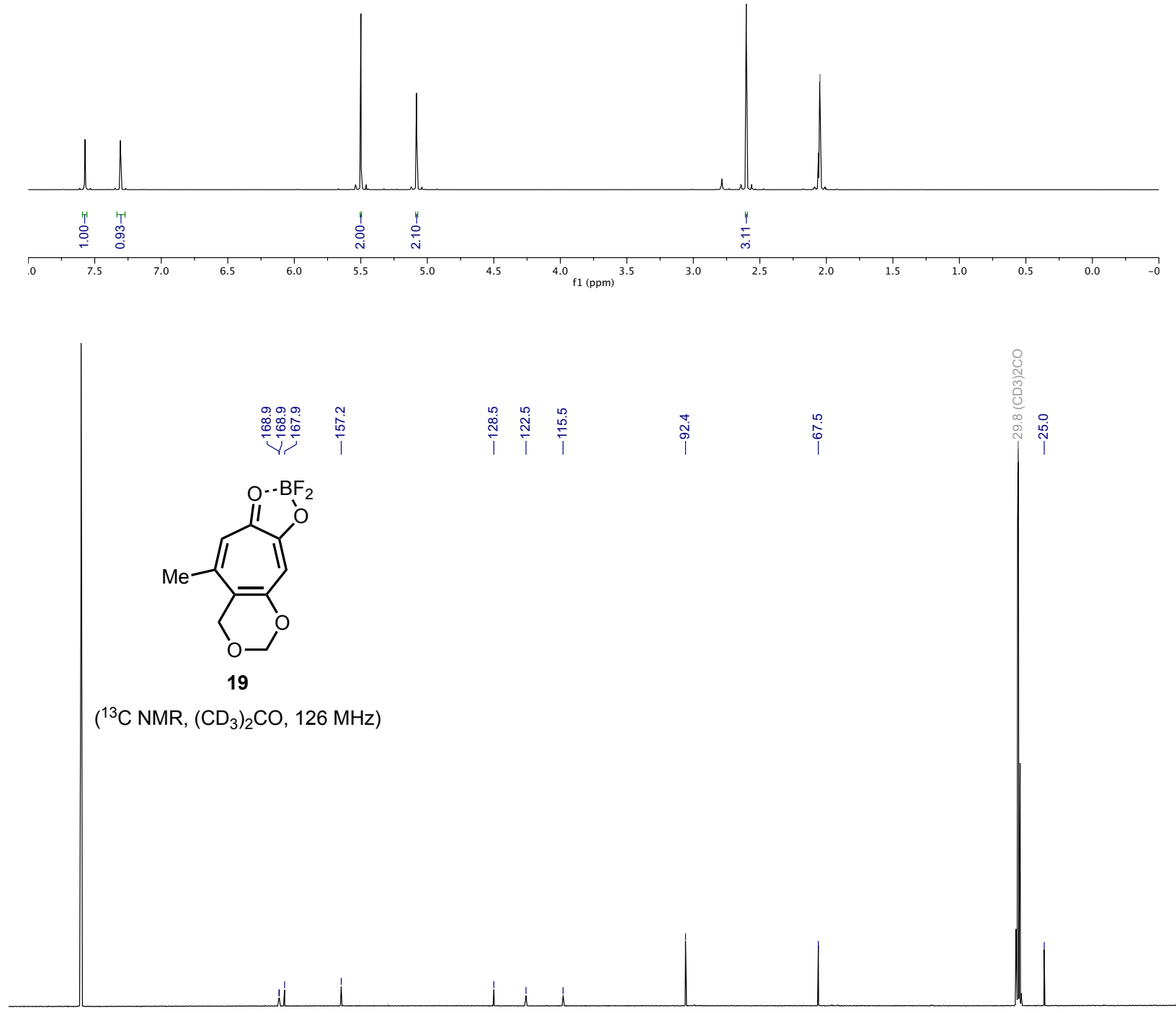

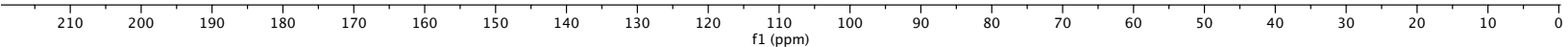




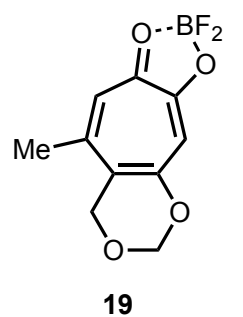

$\left({ }^{19} \mathrm{~F}, \mathrm{NMR},\left(\mathrm{CD}_{3}\right)_{2} \mathrm{CO}, 470 \mathrm{MHz}\right)$

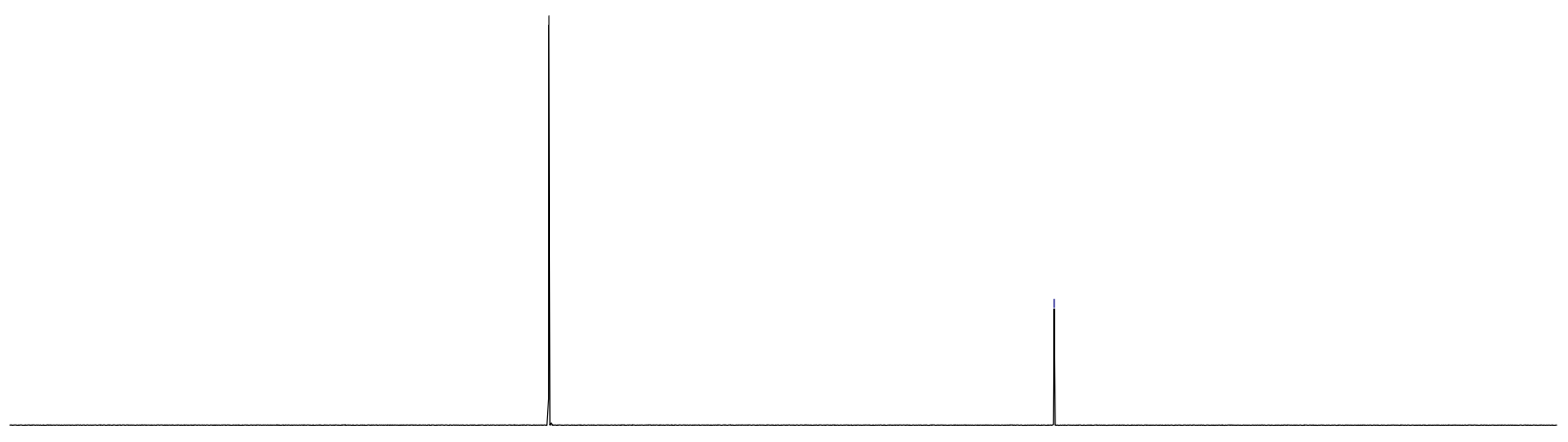

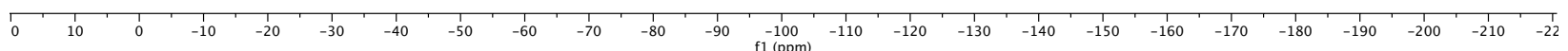




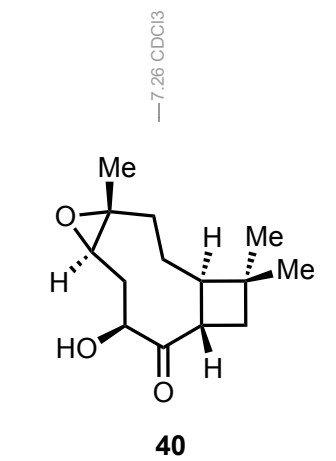

( ${ }^{1} \mathrm{H}$ NMR, $\mathrm{CDCl}_{3}, 500 \mathrm{MHz}$ )

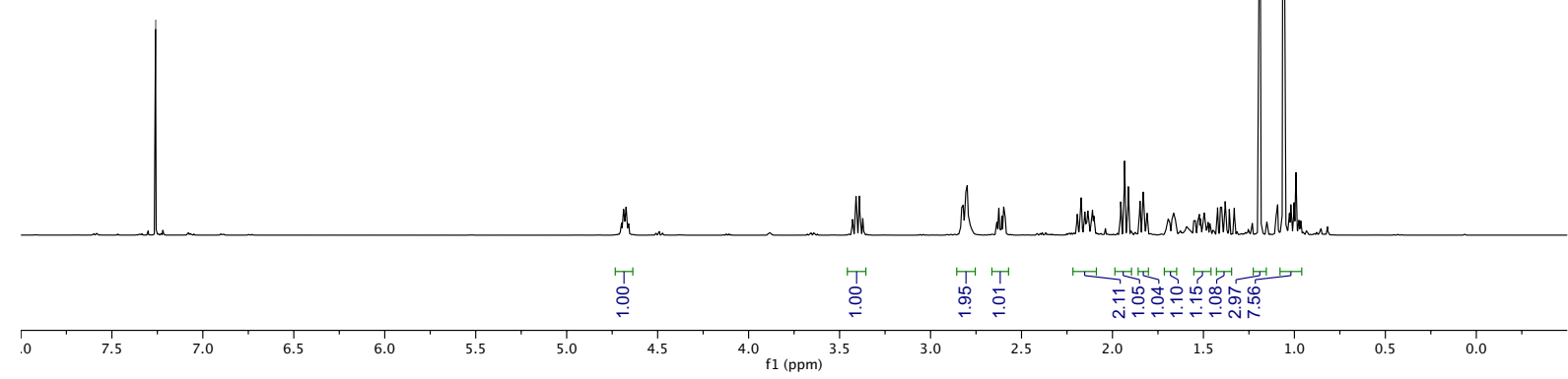

$\stackrel{\substack{n \\ \stackrel{N}{N}}}{1}$
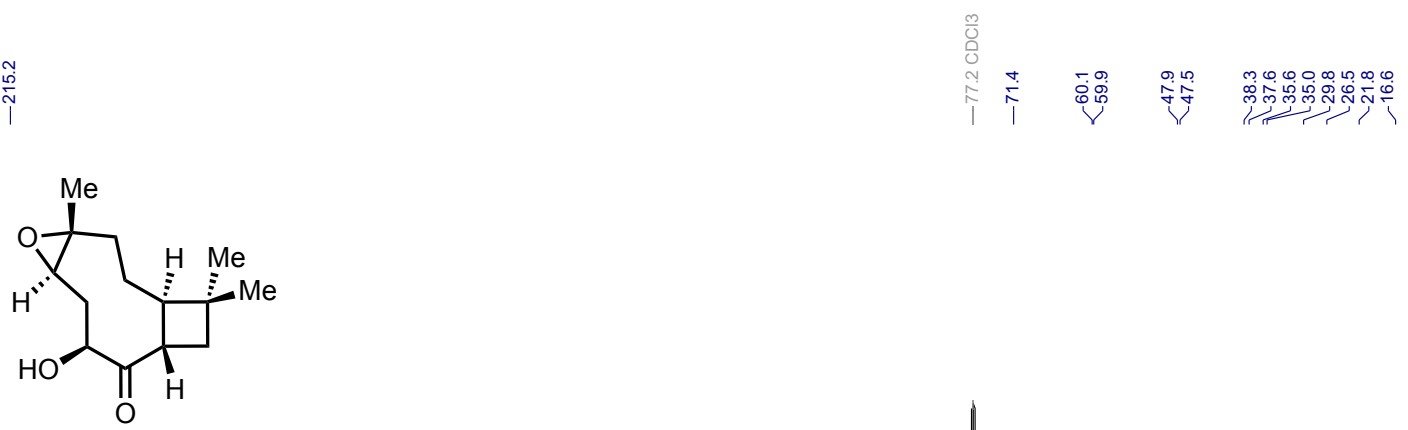

40

$\left({ }^{13} \mathrm{C} \mathrm{NMR}, \mathrm{CDCl}_{3}, 126 \mathrm{MHz}\right)$
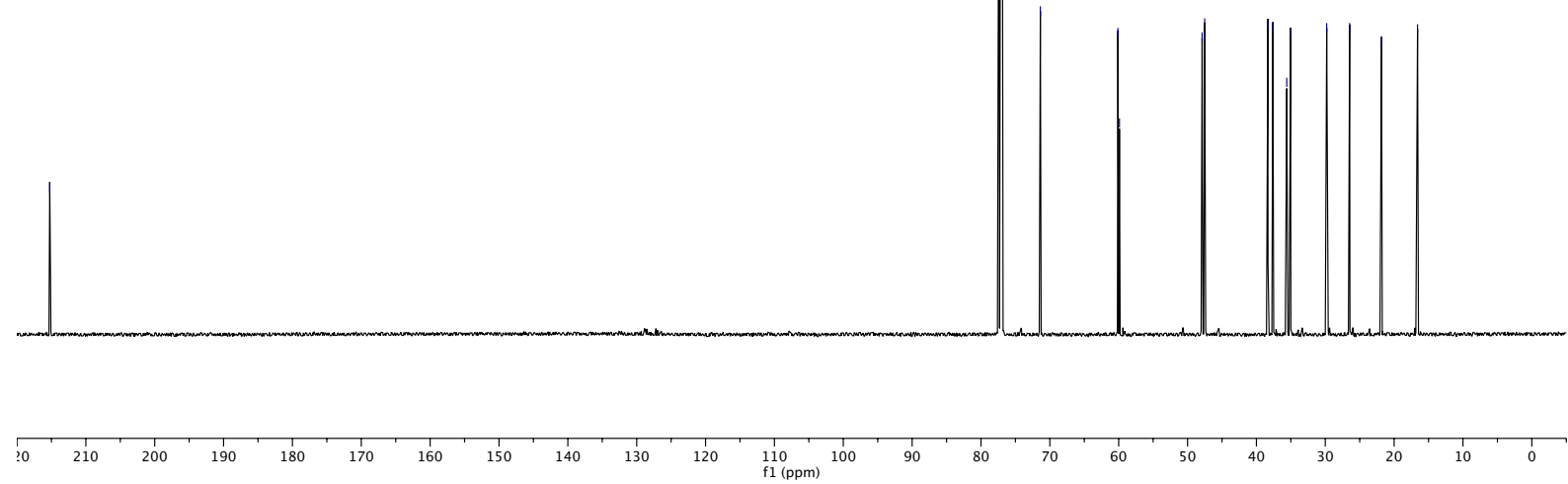

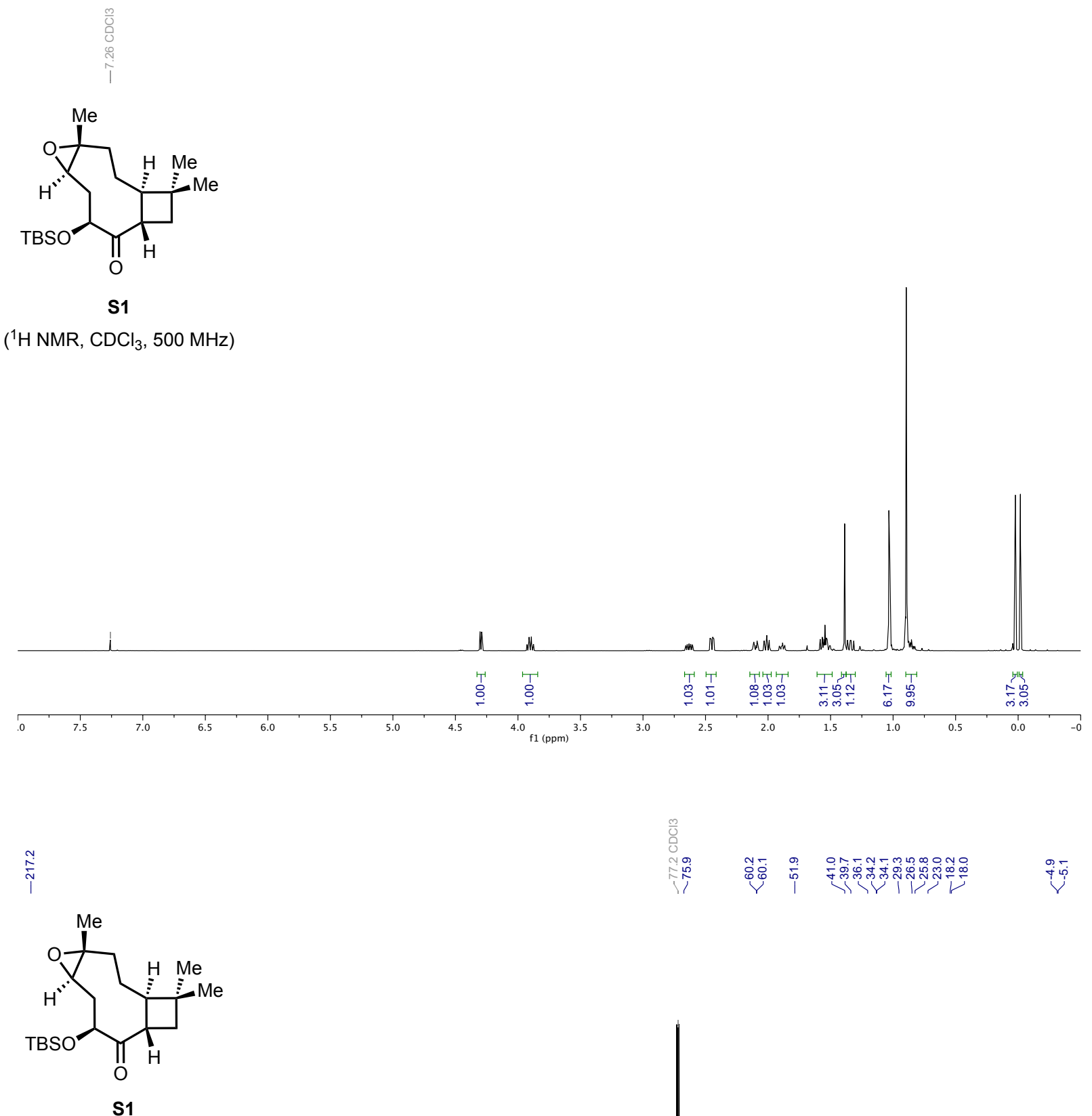

$\left({ }^{13} \mathrm{C} \mathrm{NMR}, \mathrm{CDCl}_{3}, 126 \mathrm{MHz}\right)$

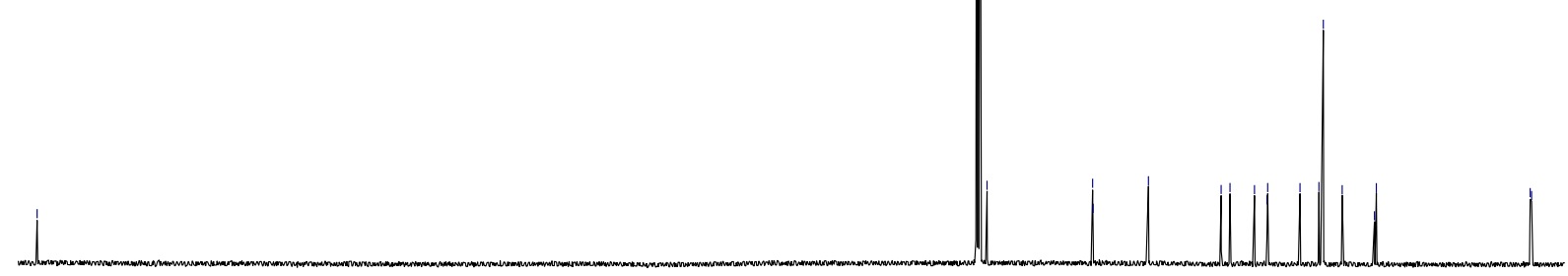

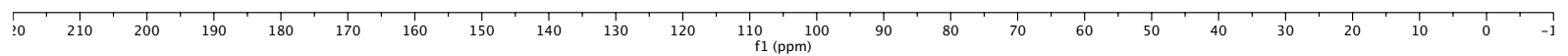




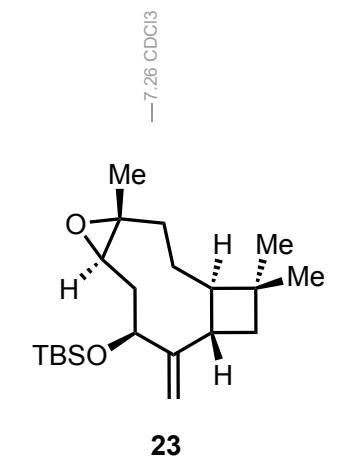

$\left({ }^{1} \mathrm{H} \mathrm{NMR}, \mathrm{CDCl}_{3}, 500 \mathrm{MHz}\right)$
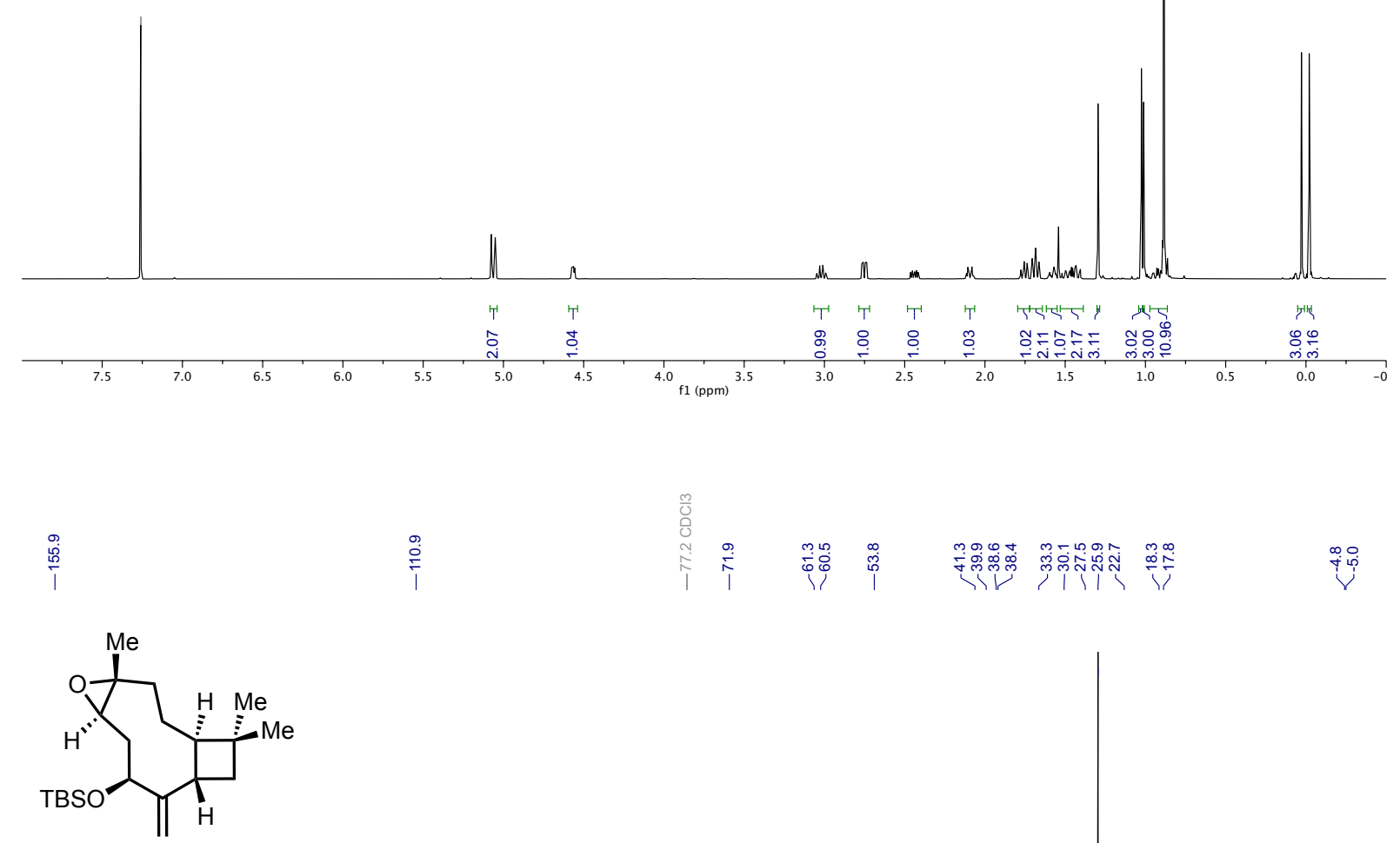

23

$\left({ }^{13} \mathrm{C} \mathrm{NMR}, \mathrm{CDCl}_{3}, 126 \mathrm{MHz}\right)$

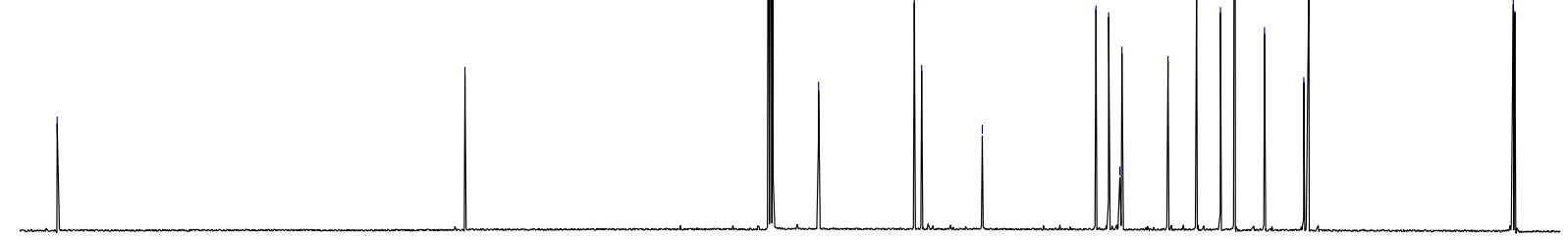

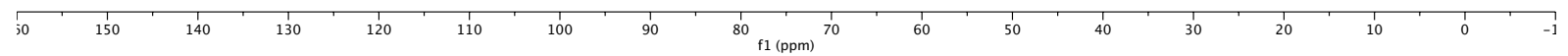




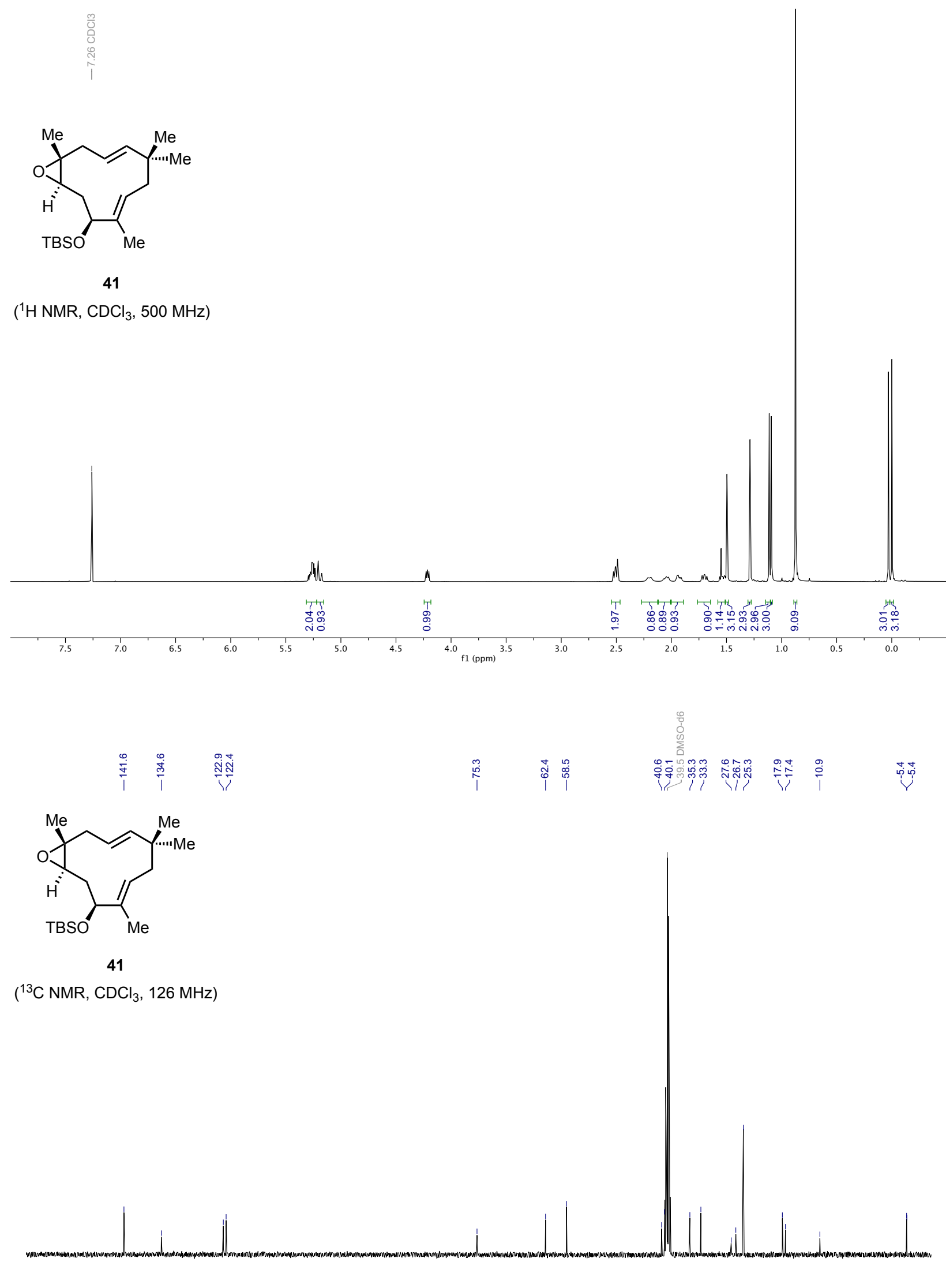

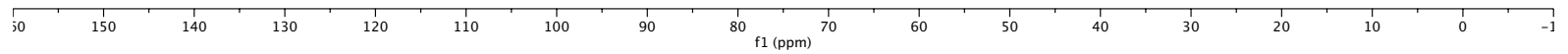



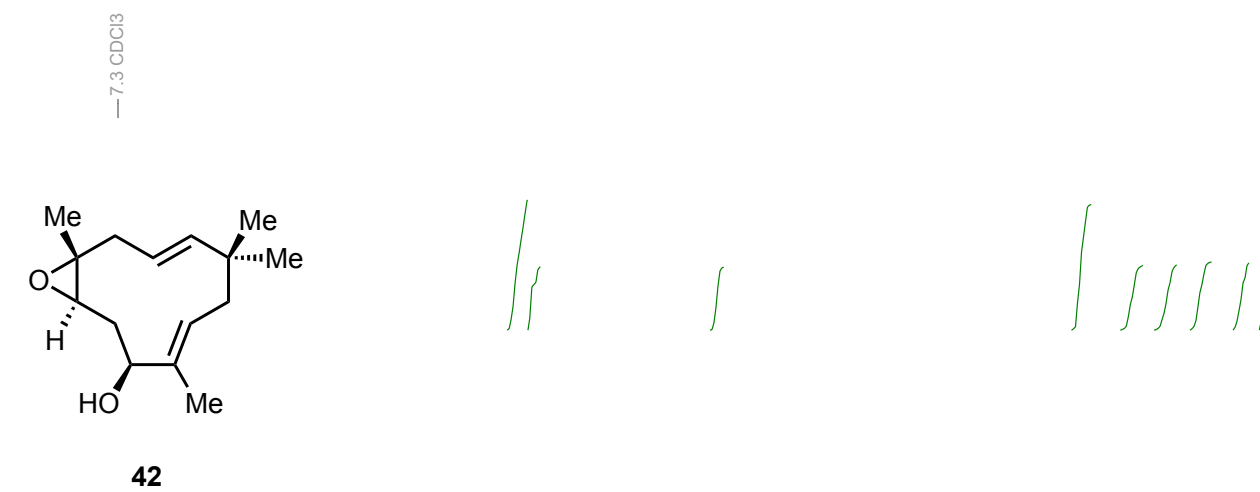

$\left({ }^{1} \mathrm{H} \mathrm{NMR}, \mathrm{CDCl}_{3}, 500 \mathrm{MHz}\right)$
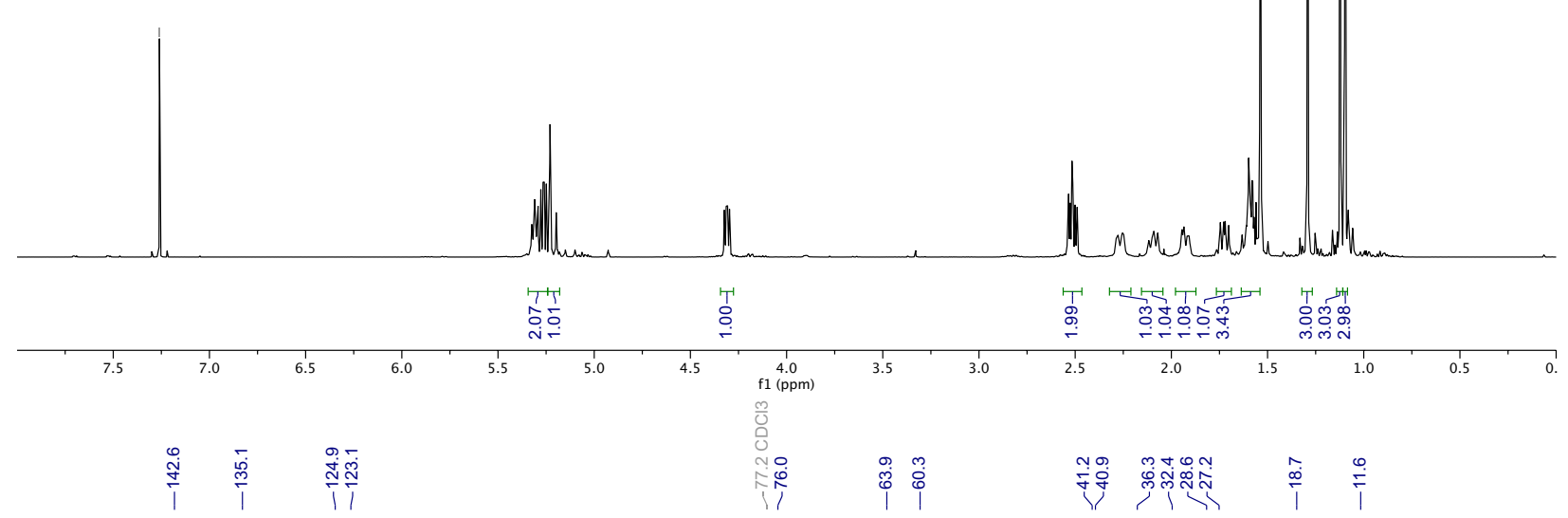

产

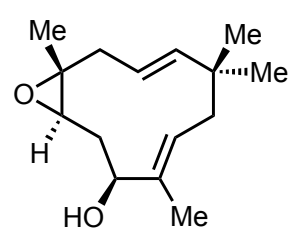

42

$\left({ }^{13} \mathrm{C} \mathrm{NMR}, \mathrm{CDCl}_{3}, 126 \mathrm{MHz}\right)$

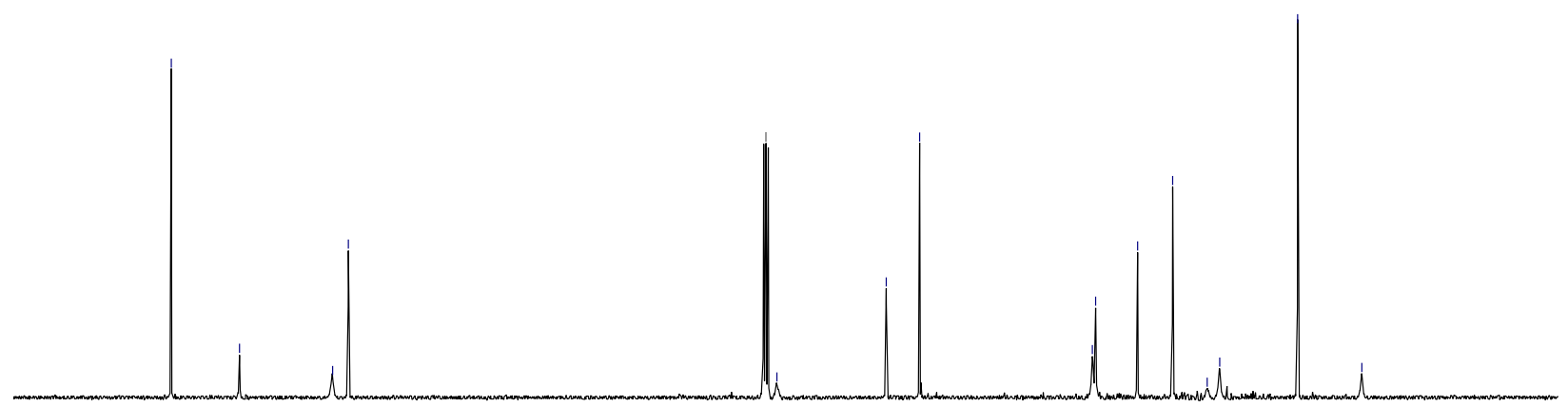

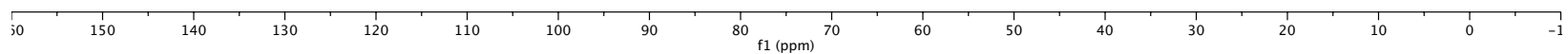



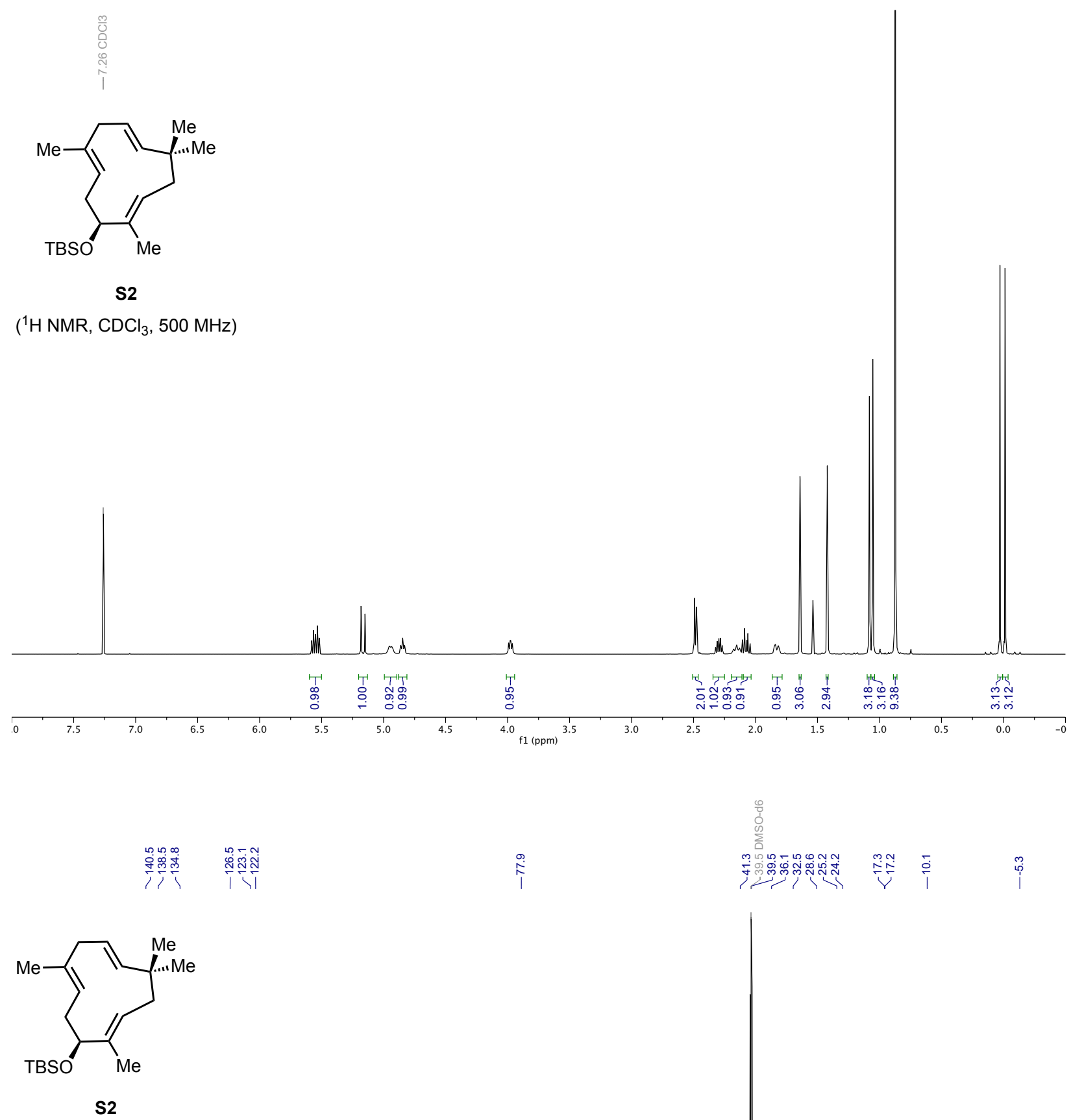

$\left({ }^{13} \mathrm{C}\right.$ NMR, $\left(\mathrm{CD}_{3}\right)_{2} \mathrm{SO}$,

$80^{\circ} \mathrm{C}, 126 \mathrm{MHz}$ )
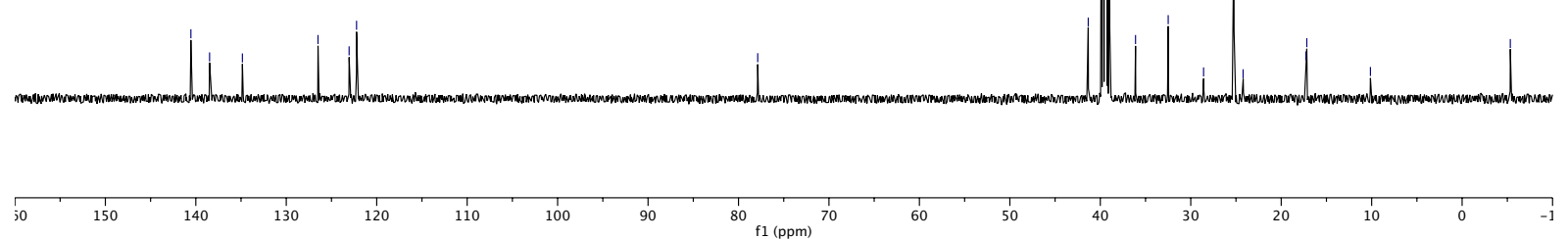


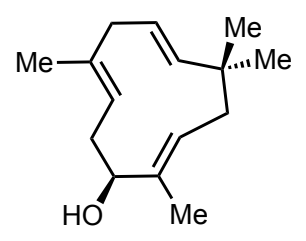

11

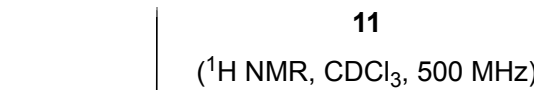

$\left({ }^{1} \mathrm{H} \mathrm{NMR}, \mathrm{CDCl}_{3}, 500 \mathrm{MHz}\right)$
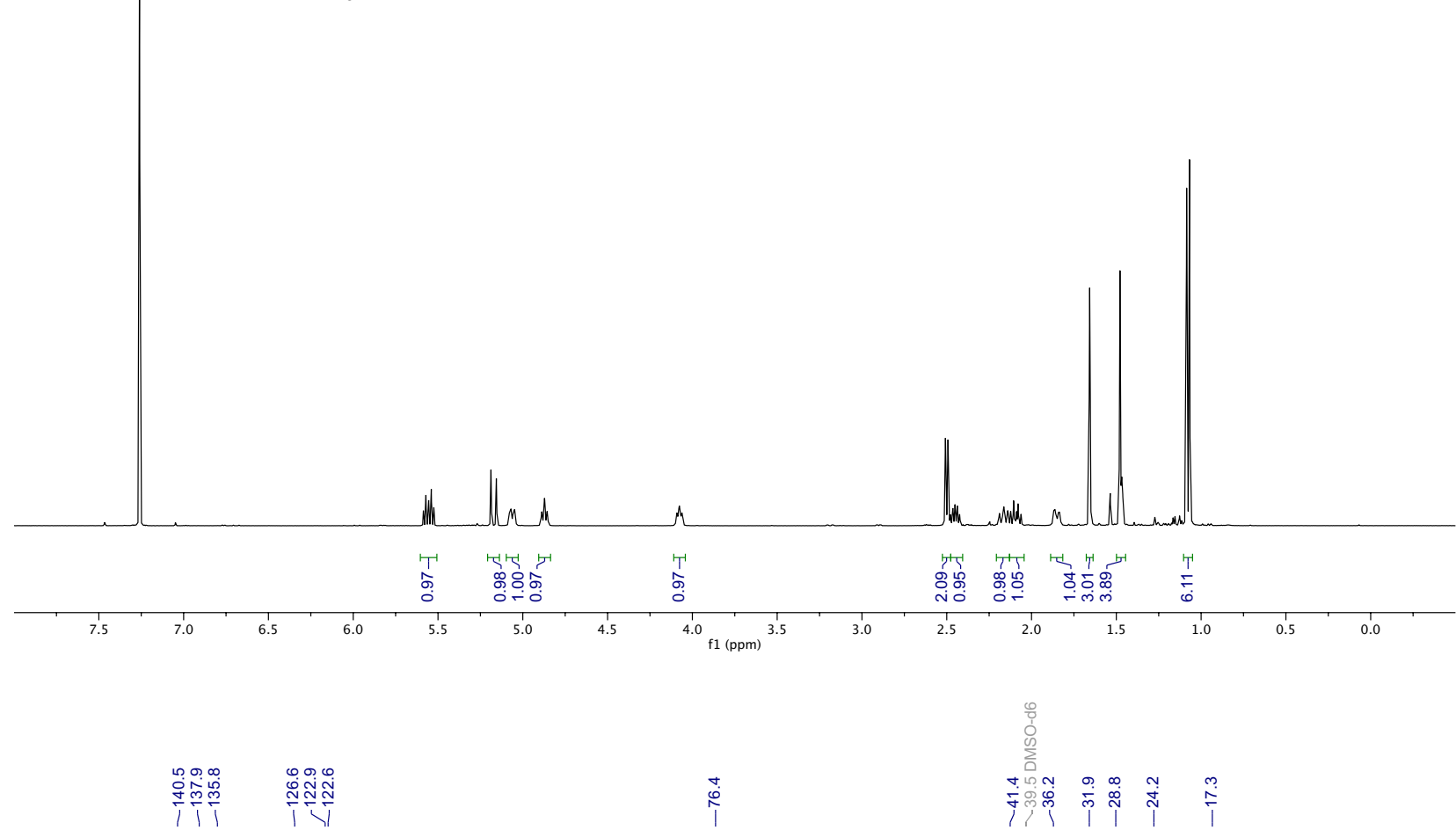

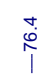
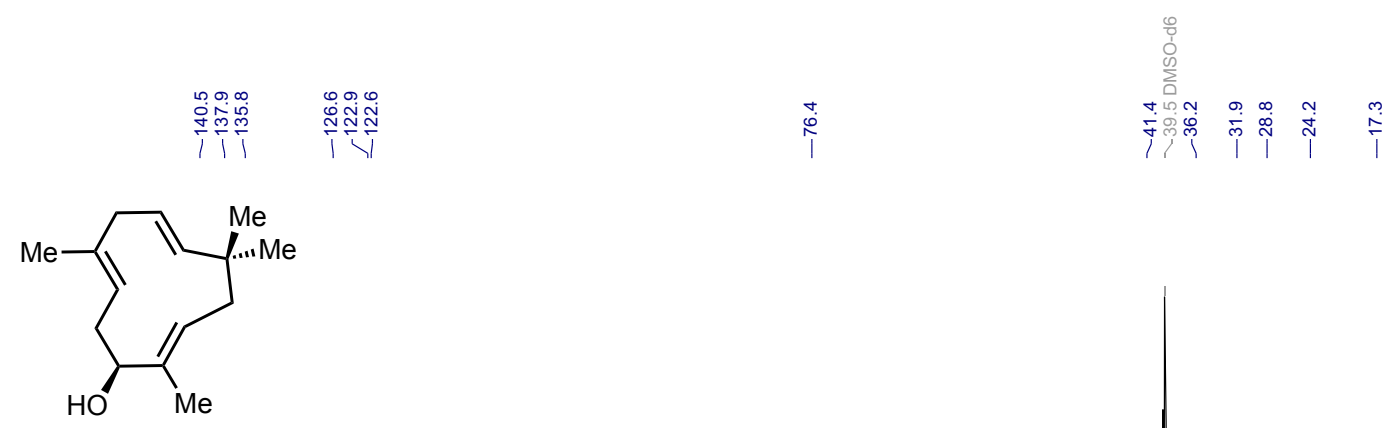

11

$\left({ }^{13} \mathrm{C} \mathrm{NMR},\left(\mathrm{CD}_{3}\right)_{2} \mathrm{SO}\right.$

$\left.90{ }^{\circ} \mathrm{C}, 126 \mathrm{MHz}\right)$

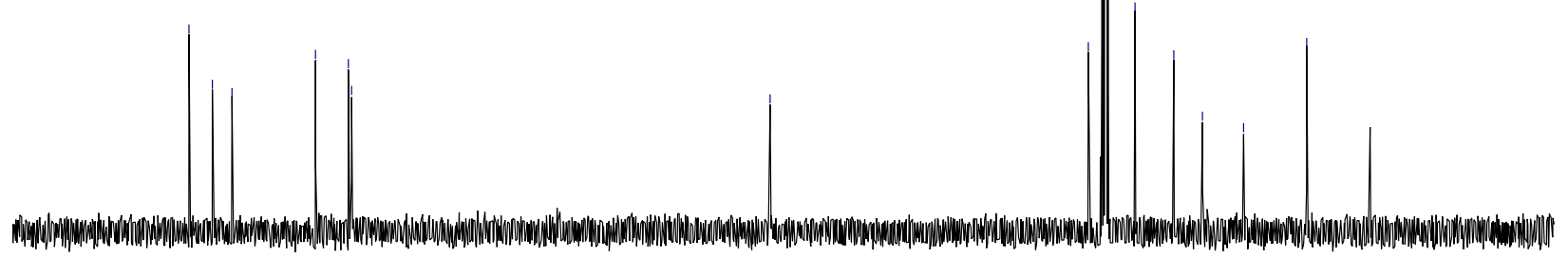

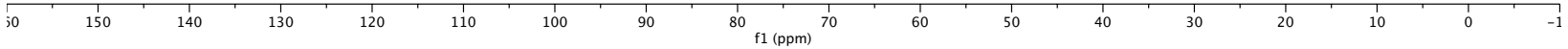




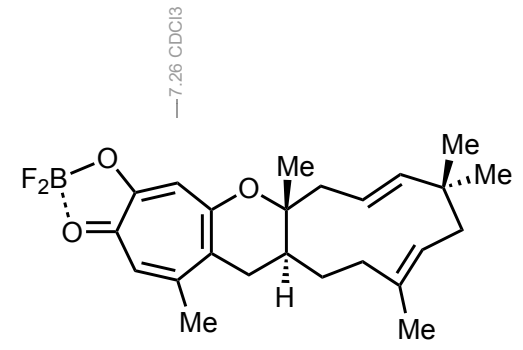

43

$\left({ }^{1} \mathrm{H} \mathrm{NMR}, \mathrm{CDCl}_{3}, 500 \mathrm{MHz}\right.$ )

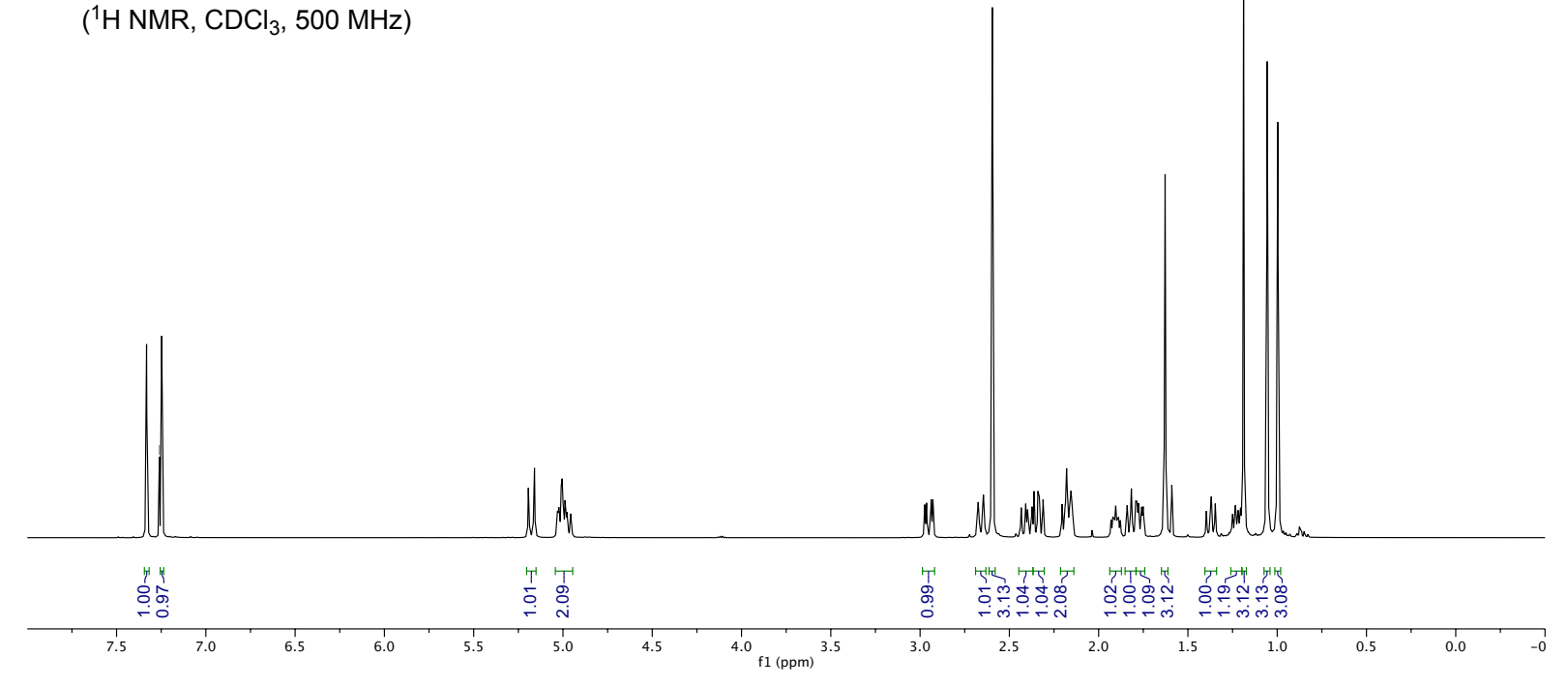

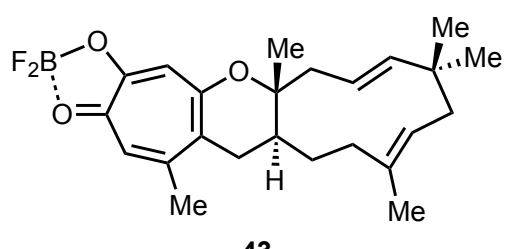

$\left({ }^{13} \mathrm{C} \mathrm{NMR}, \mathrm{CDCl}_{3}, 126 \mathrm{MHz}\right)$
任

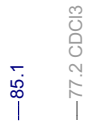

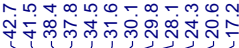

।

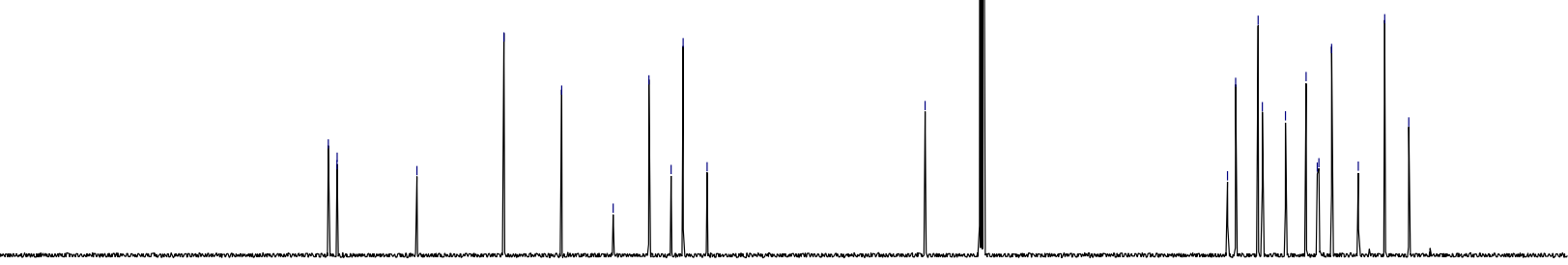

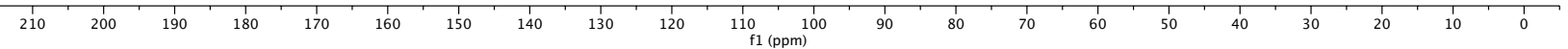




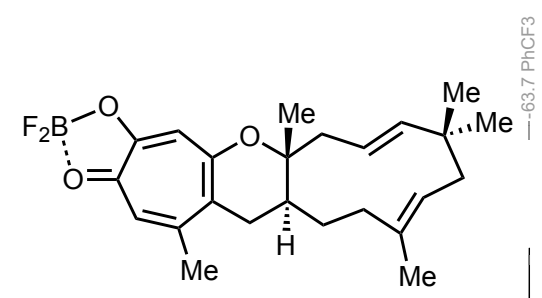

43

$\left({ }^{19} \mathrm{~F} \mathrm{NMR}, \mathrm{CDCl}_{3}, 470 \mathrm{MHz}\right)$

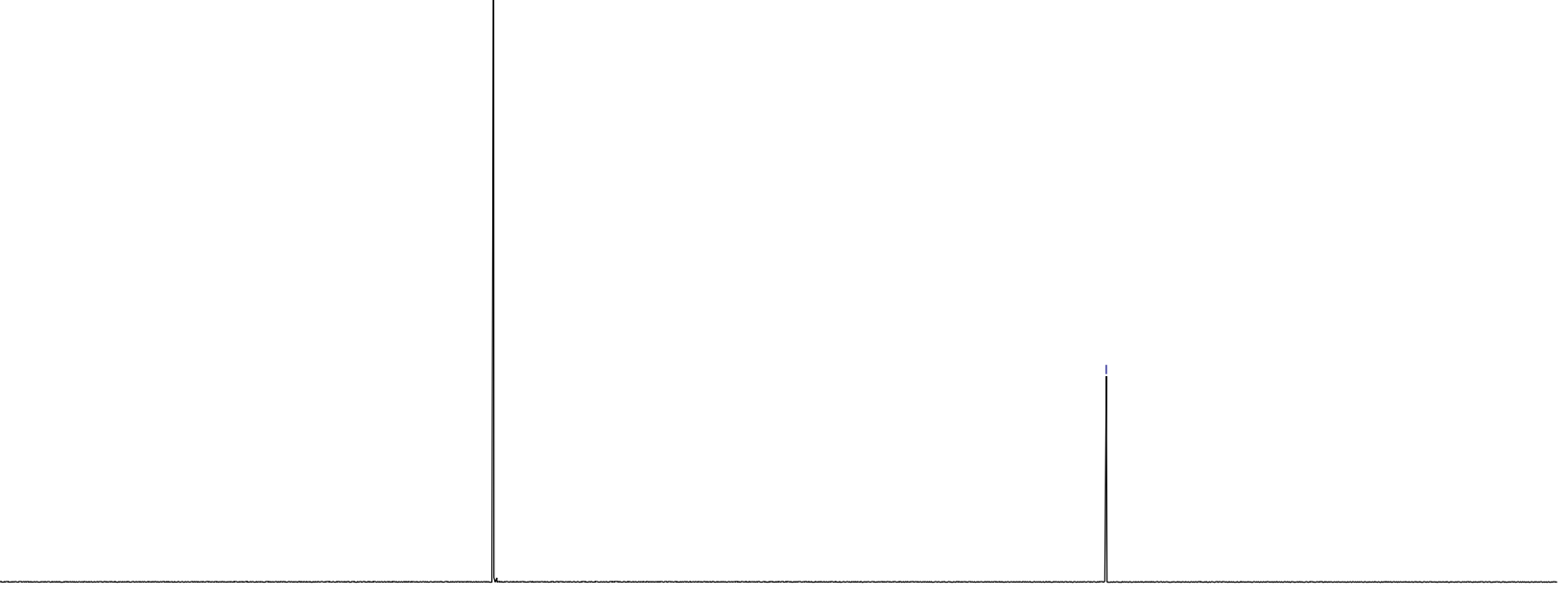

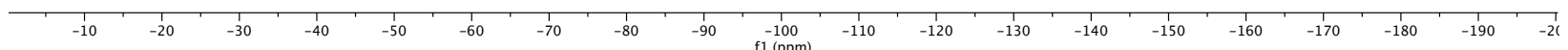




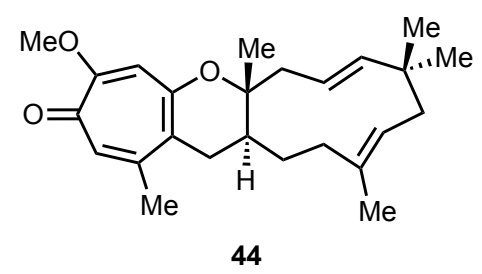

$\left({ }^{1} \mathrm{H} \mathrm{NMR}, \mathrm{CDCl}_{3}, 500 \mathrm{MHz}\right)$
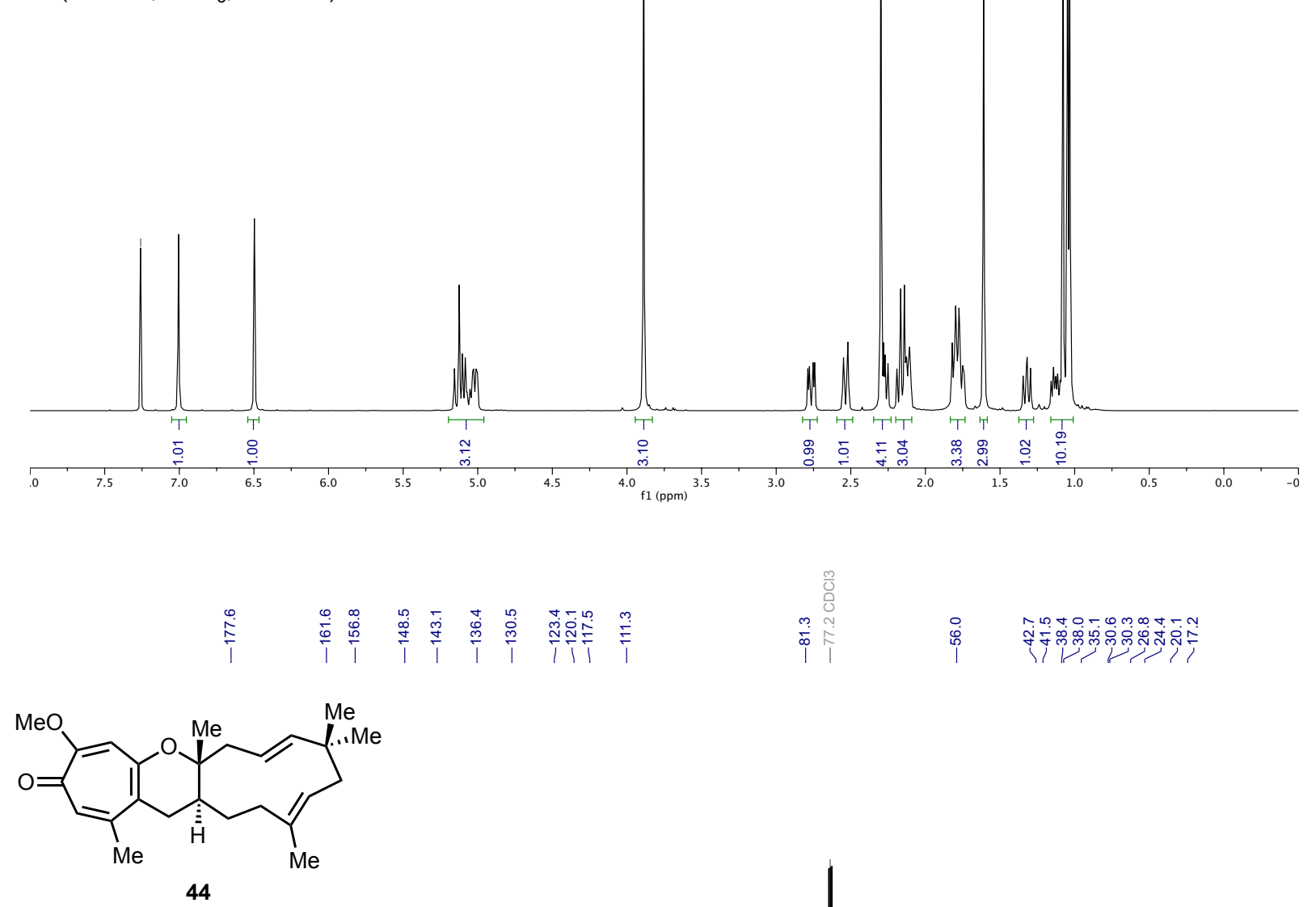

$\left({ }^{13} \mathrm{C} \mathrm{NMR}, \mathrm{CDCl}_{3}, 126 \mathrm{MHz}\right)$
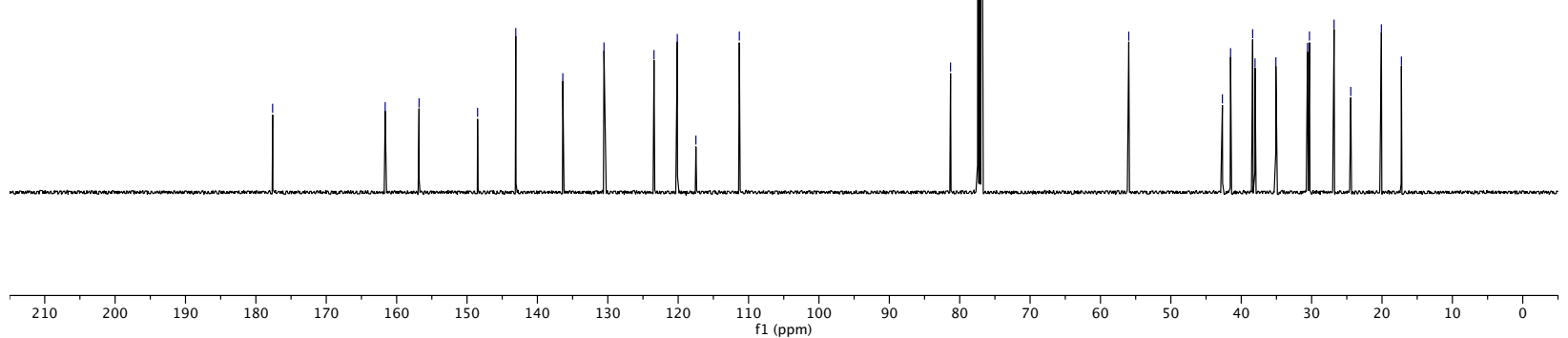


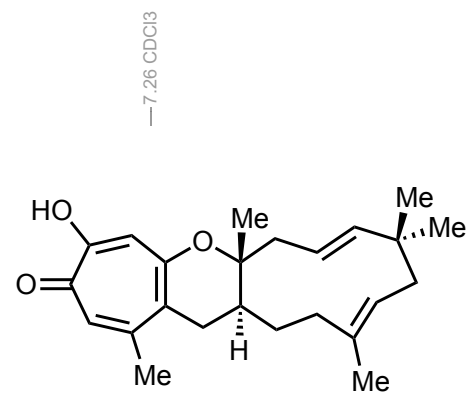

(士)-dehydroxyepolone B (15')

$\left({ }^{1} \mathrm{H} \mathrm{NMR}, \mathrm{CDCl}_{3}, 500 \mathrm{MHz}\right)$

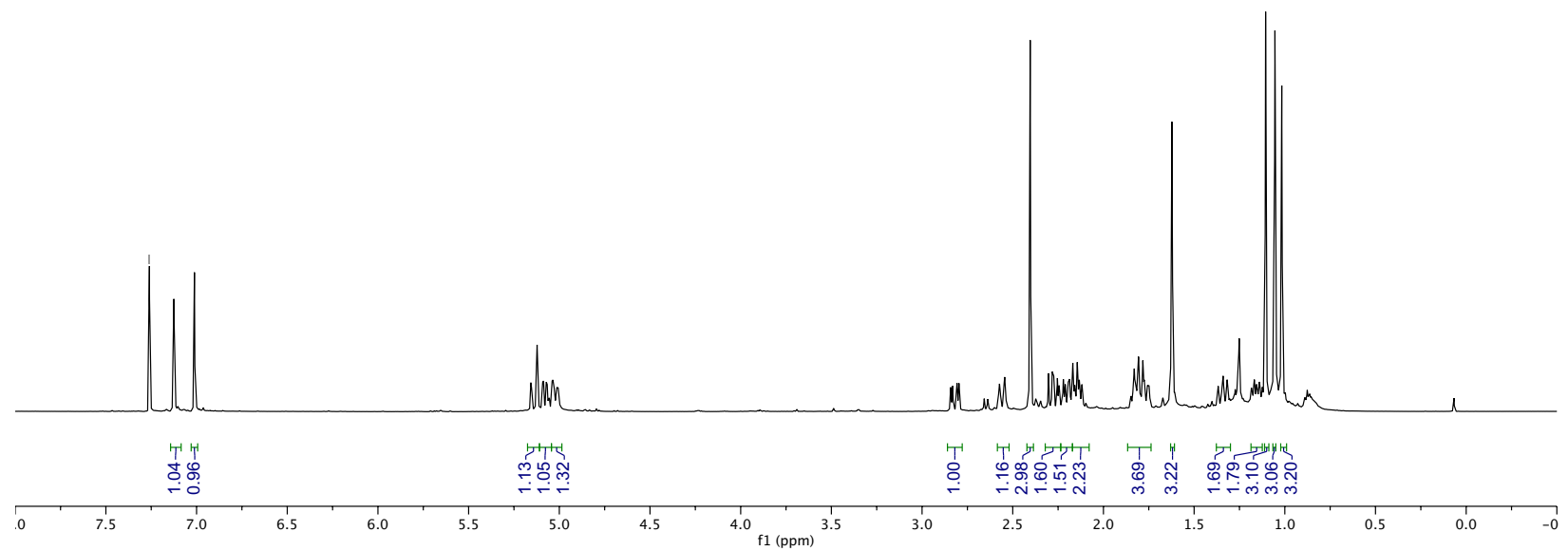

$$
\text { }
$$

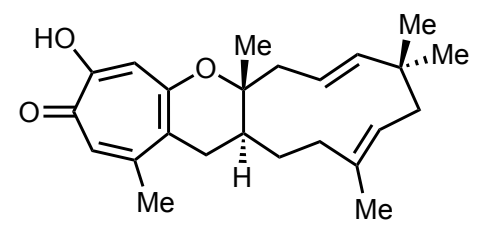

( \pm )-dehydroxyepolone B (15')

$\left({ }^{13} \mathrm{C} \mathrm{NMR}, \mathrm{CDCl}_{3}, 126 \mathrm{MHz}\right)$
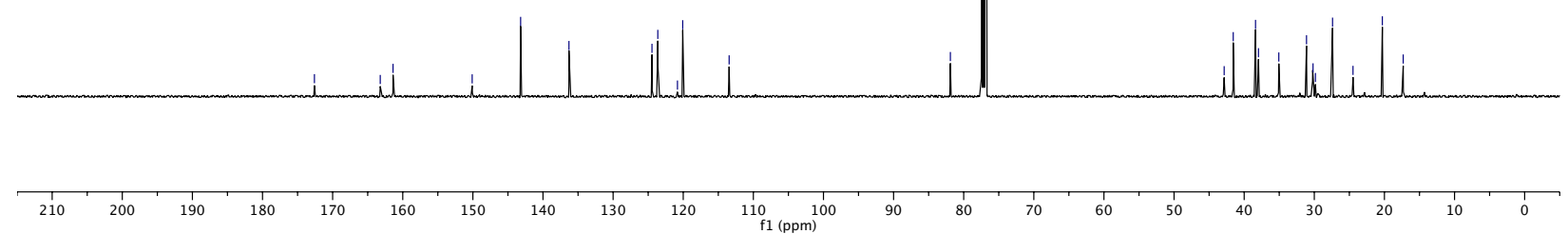

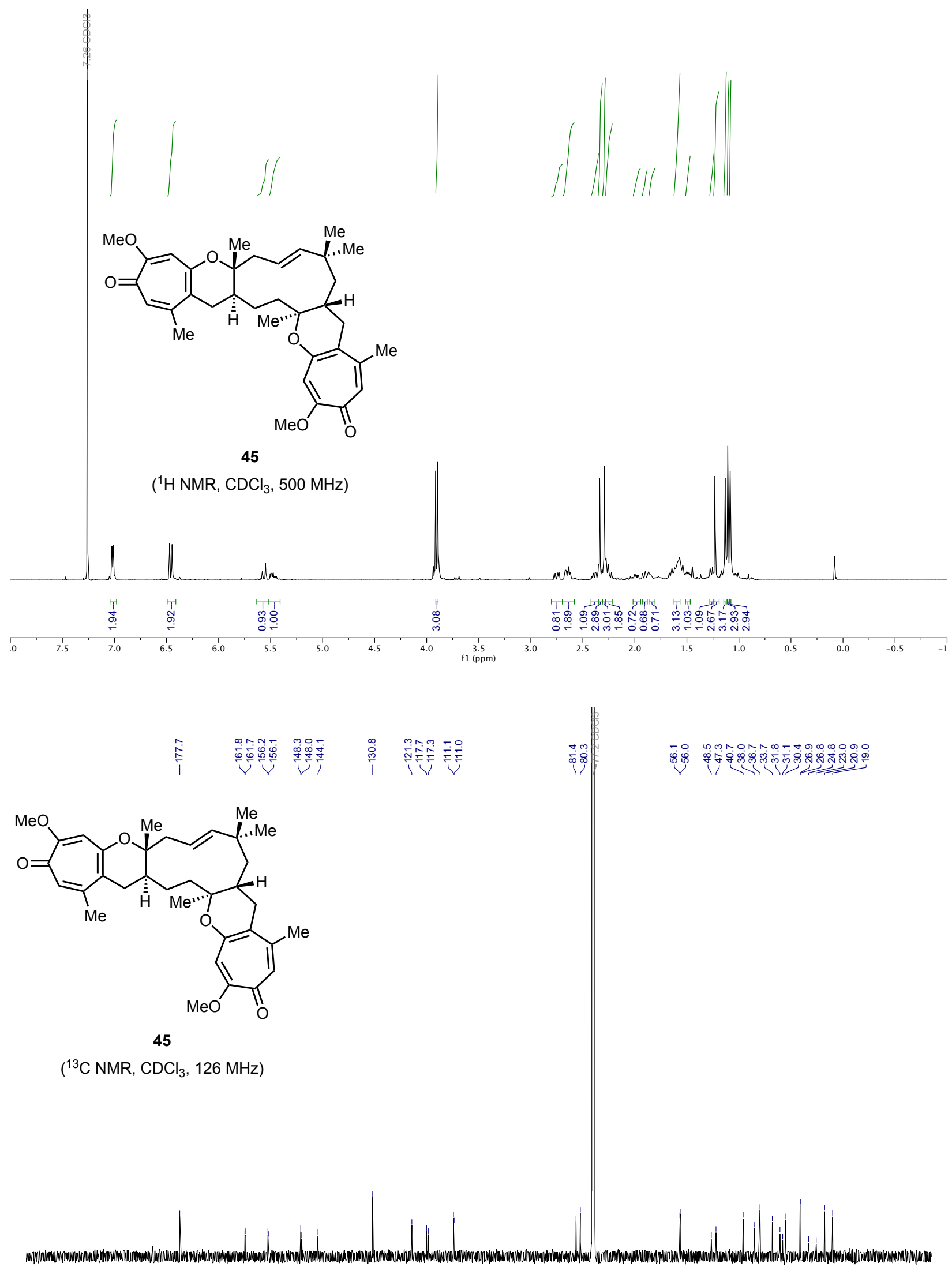

$\begin{array}{lllllllllll}210 & 200 & 190 & 180 & 170 & 160 & 150 & 140 & 130 & 120 & 110 \\ \mathrm{f} 1(\mathrm{ppm}) & 100\end{array}$ 


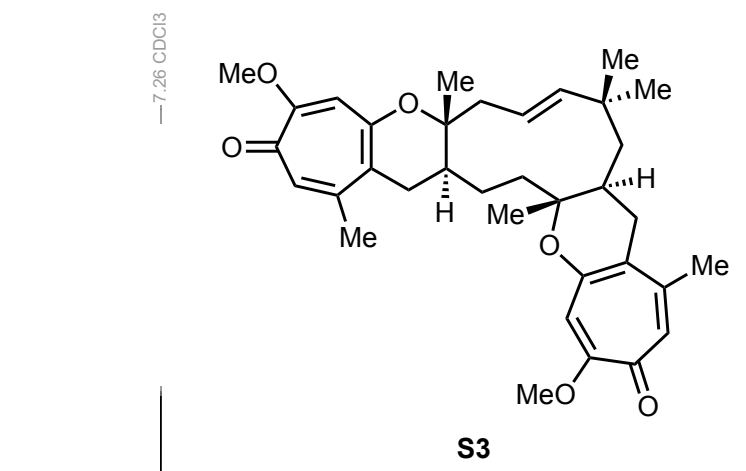

( $\left.{ }^{1} \mathrm{H} \mathrm{NMR}, \mathrm{CDCl}_{3}, 500 \mathrm{MHz}\right)$
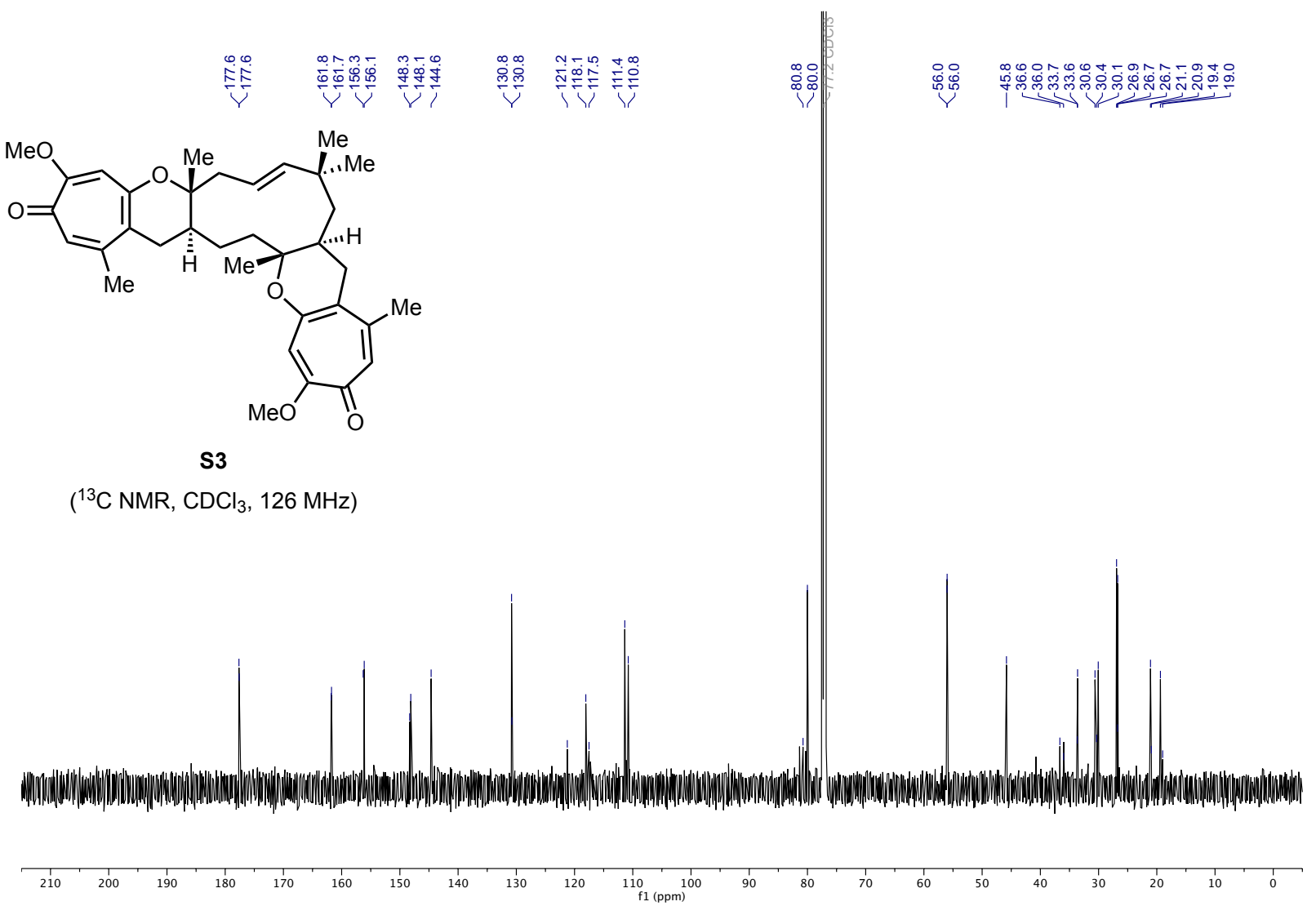

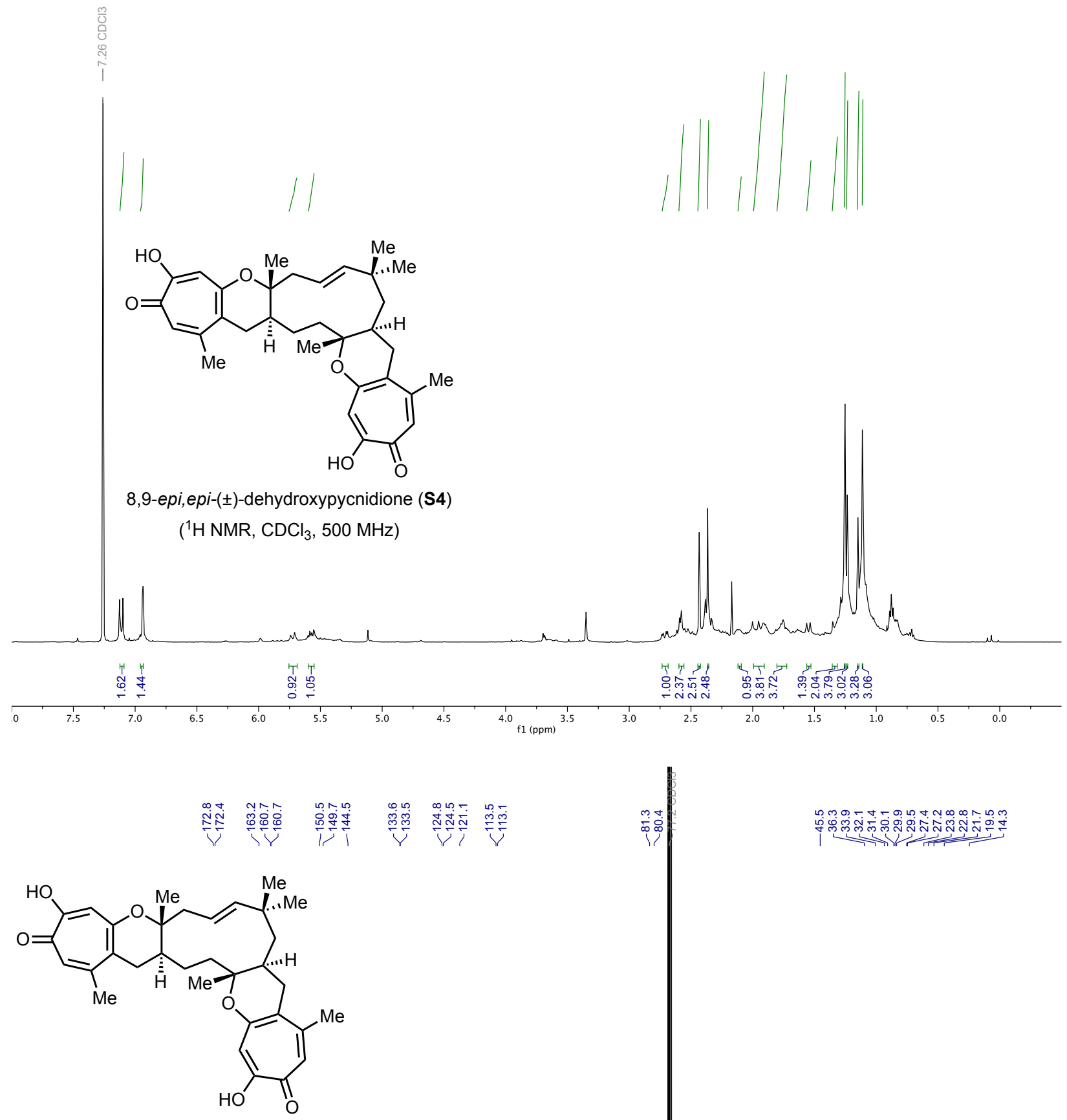

8,9-epi,epi-(土)-dehydroxypycnidione (S4)

$\left({ }^{13} \mathrm{C} \mathrm{NMR}, \mathrm{CDCl}_{3}, 126 \mathrm{MHz}\right)$

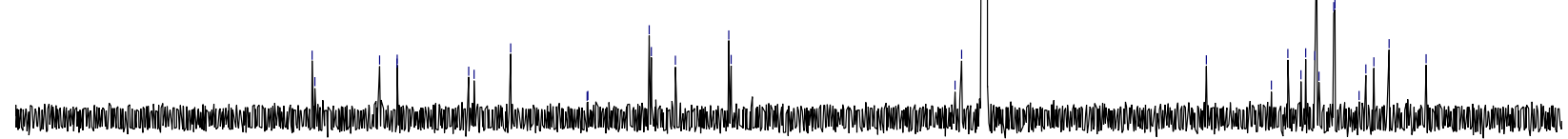

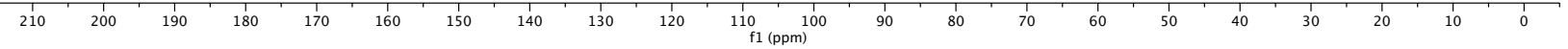



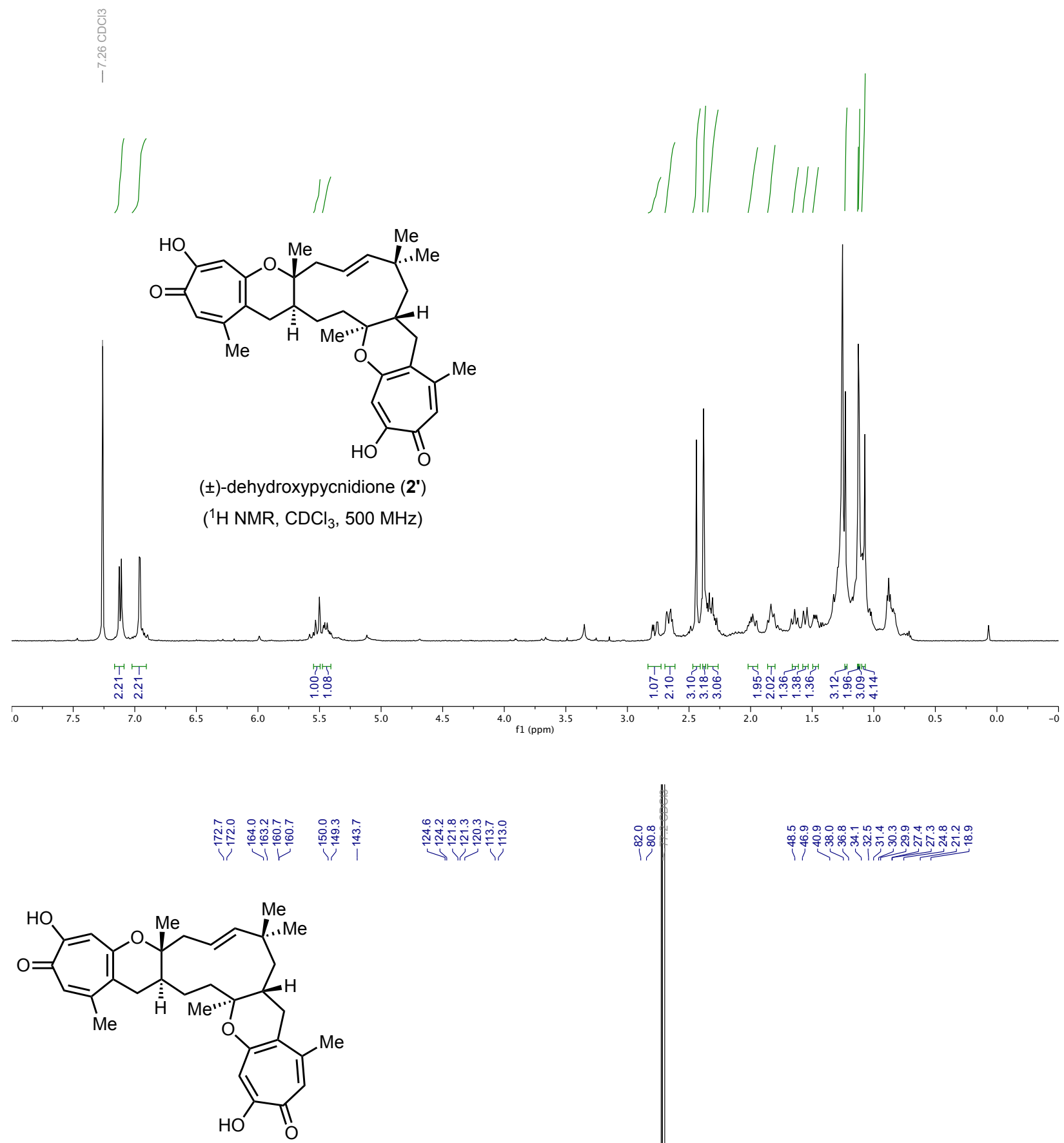

( \pm )-dehydroxypycnidione (2')

$\left({ }^{13} \mathrm{C} \mathrm{NMR}, \mathrm{CDCl}_{3}, 126 \mathrm{MHz}\right)$
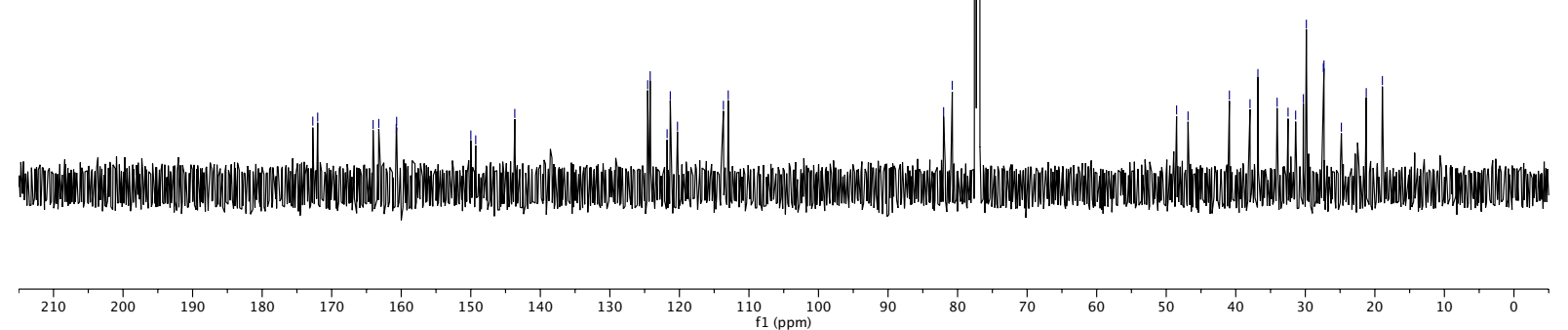


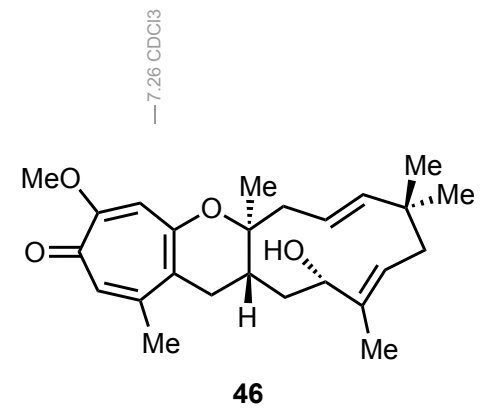

$\left({ }^{1} \mathrm{H} \mathrm{NMR}, \mathrm{CDCl}_{3}, 500 \mathrm{MHz}\right)$

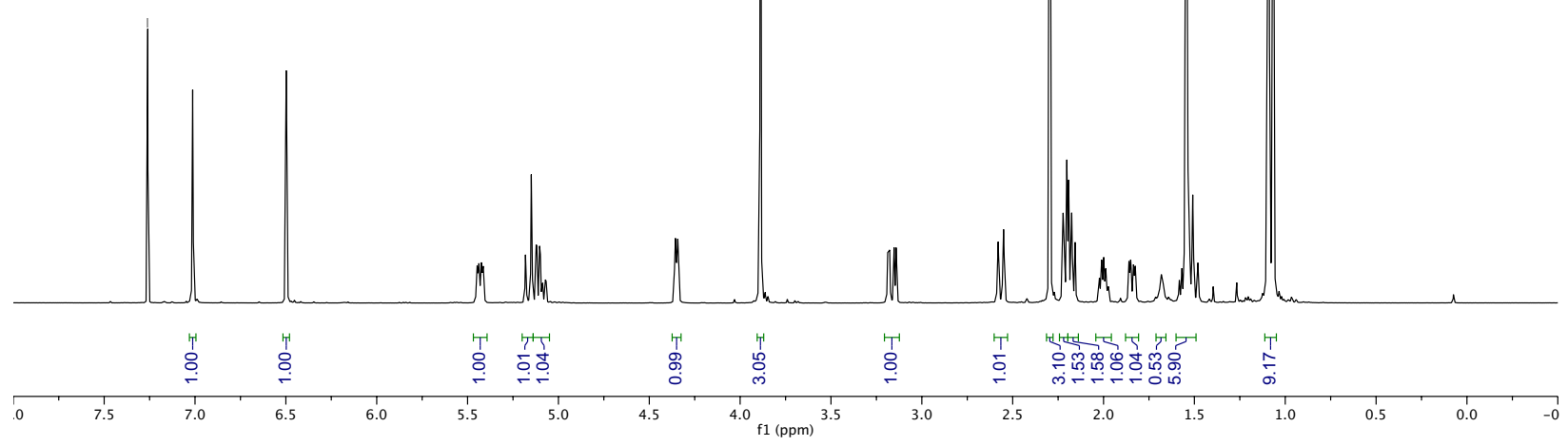

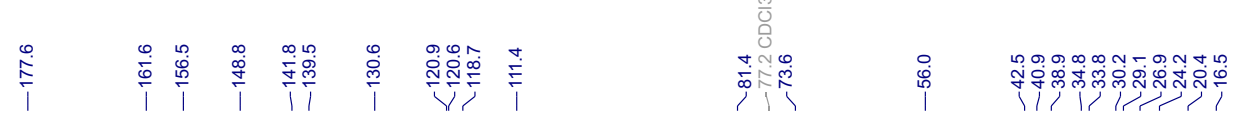

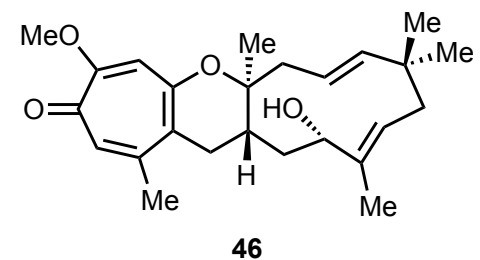

$\left({ }^{13} \mathrm{C} \mathrm{NMR}, \mathrm{CDCl}_{3}, 126 \mathrm{MHz}\right)$
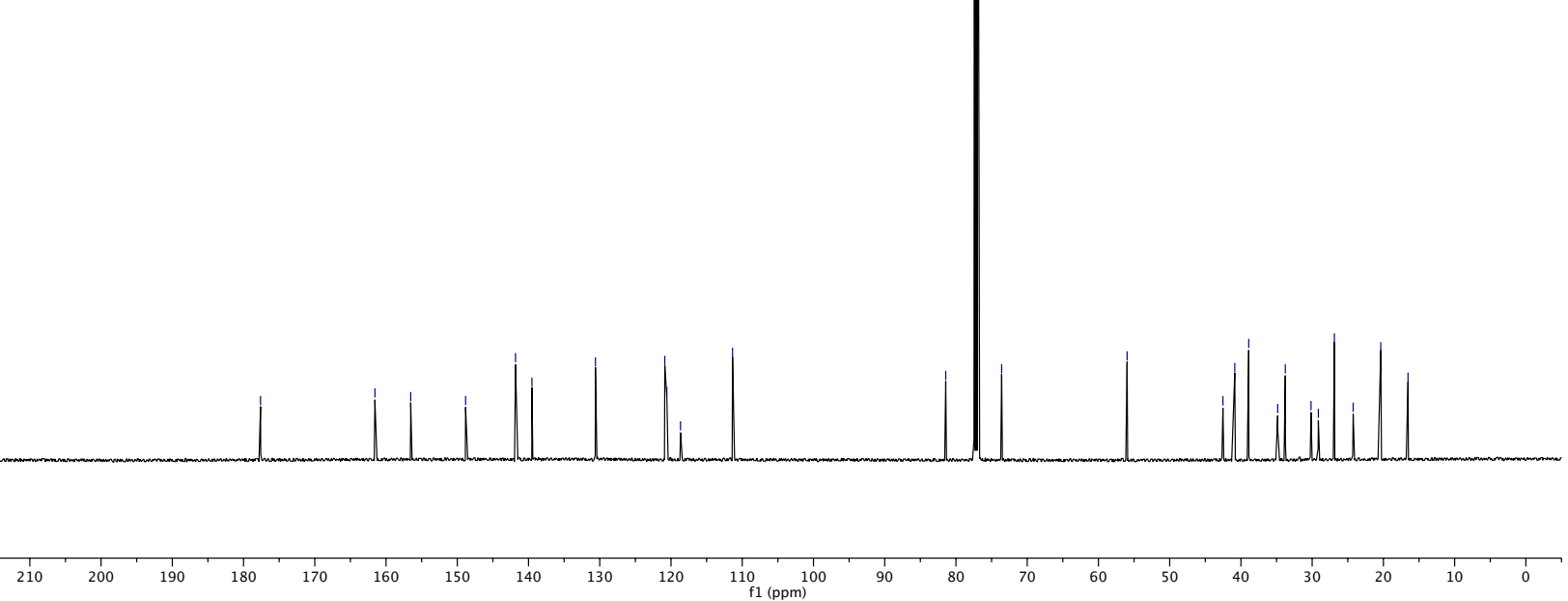


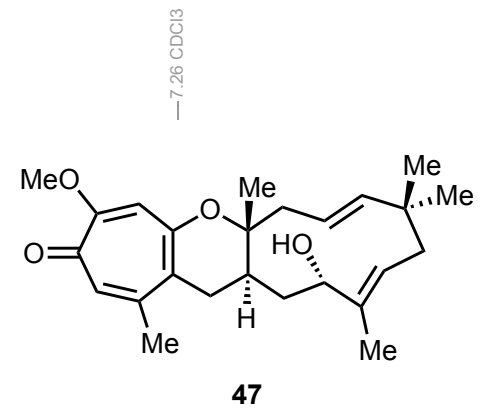

$\left({ }^{1} \mathrm{H} \mathrm{NMR}, \mathrm{CDCl}_{3}, 500 \mathrm{MHz}\right)$
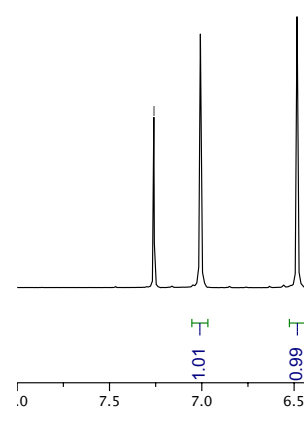

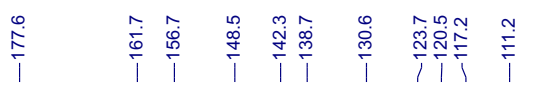

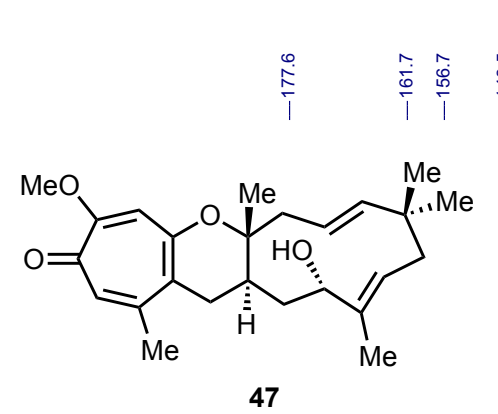

$\left({ }^{13} \mathrm{C} \mathrm{NMR}, \mathrm{CDCl}_{3}, 126 \mathrm{MHz}\right)$
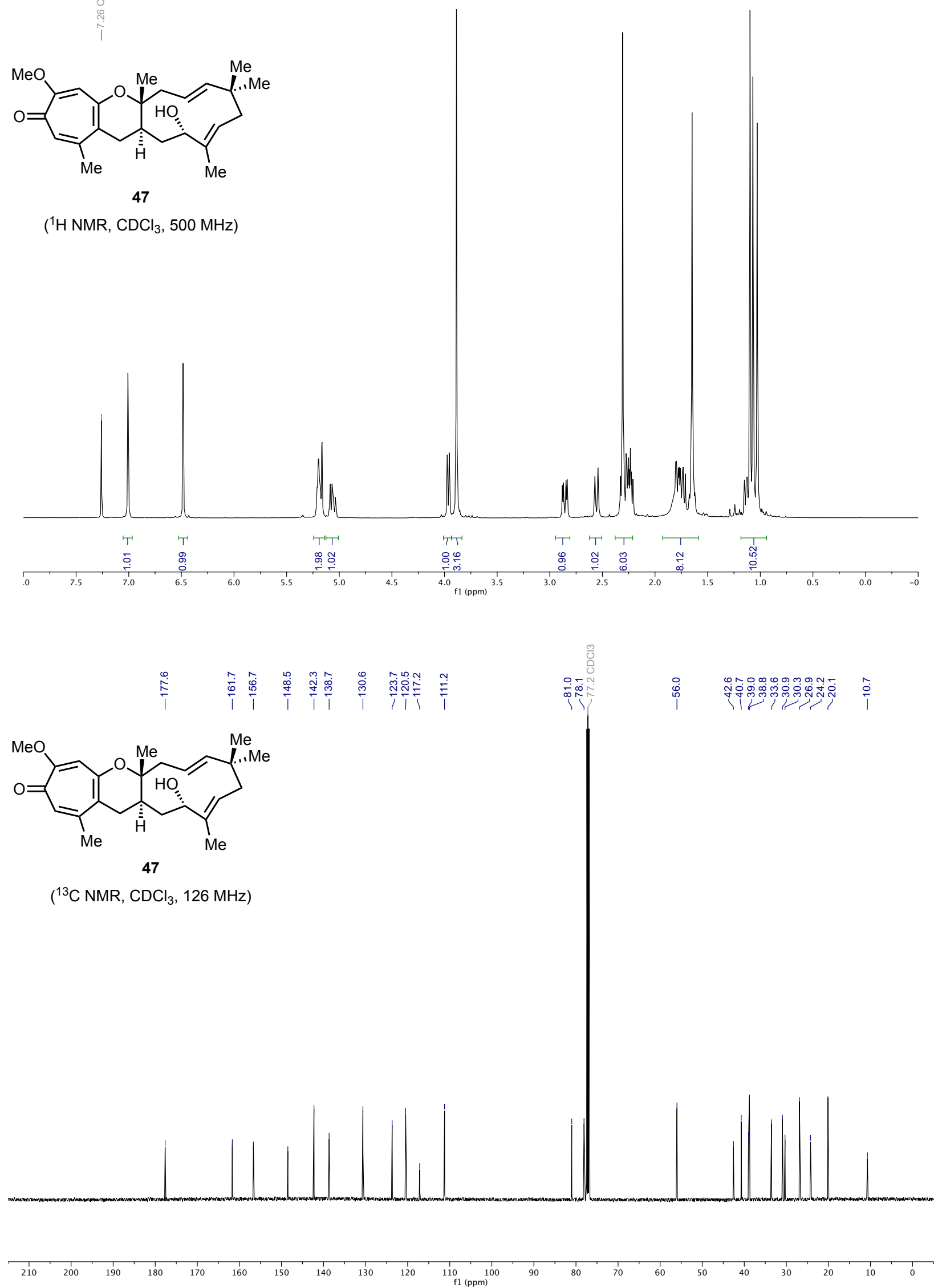


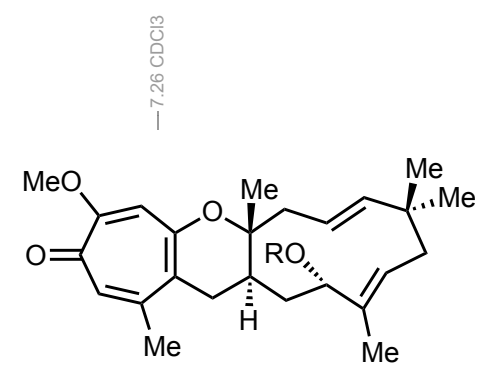

48: $\mathrm{R}=p-\mathrm{BrC}_{6} \mathrm{H}_{4} \mathrm{NHCO}$

( ${ }^{1} \mathrm{H} \mathrm{NMR}, \mathrm{CDCl}_{3}, 500 \mathrm{MHz}$ )
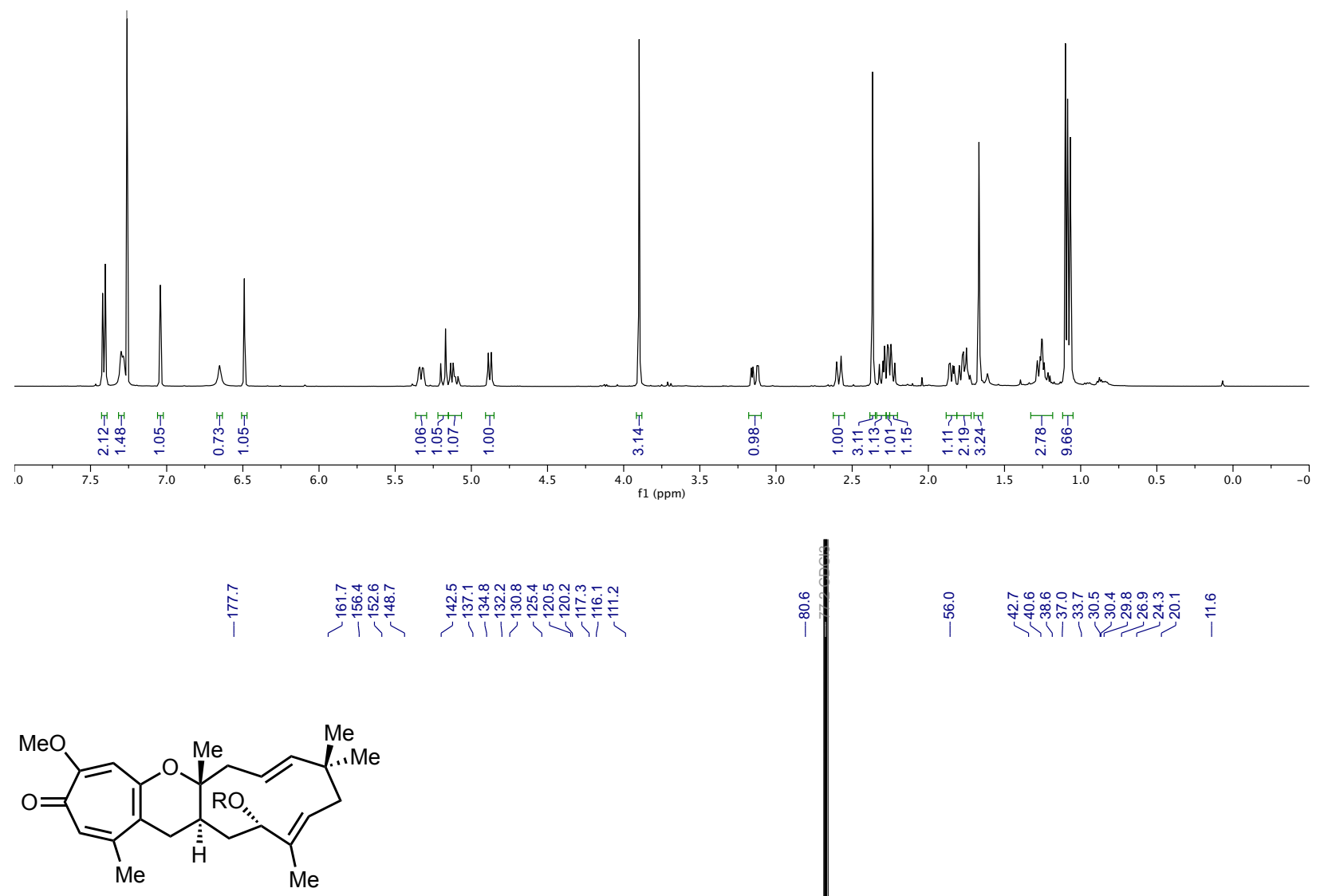

48: $\mathrm{R}=p-\mathrm{BrC}_{6} \mathrm{H}_{4} \mathrm{NHCO}$

$\left({ }^{13} \mathrm{C} \mathrm{NMR}, \mathrm{CDCl}_{3}, 126 \mathrm{MHz}\right)$
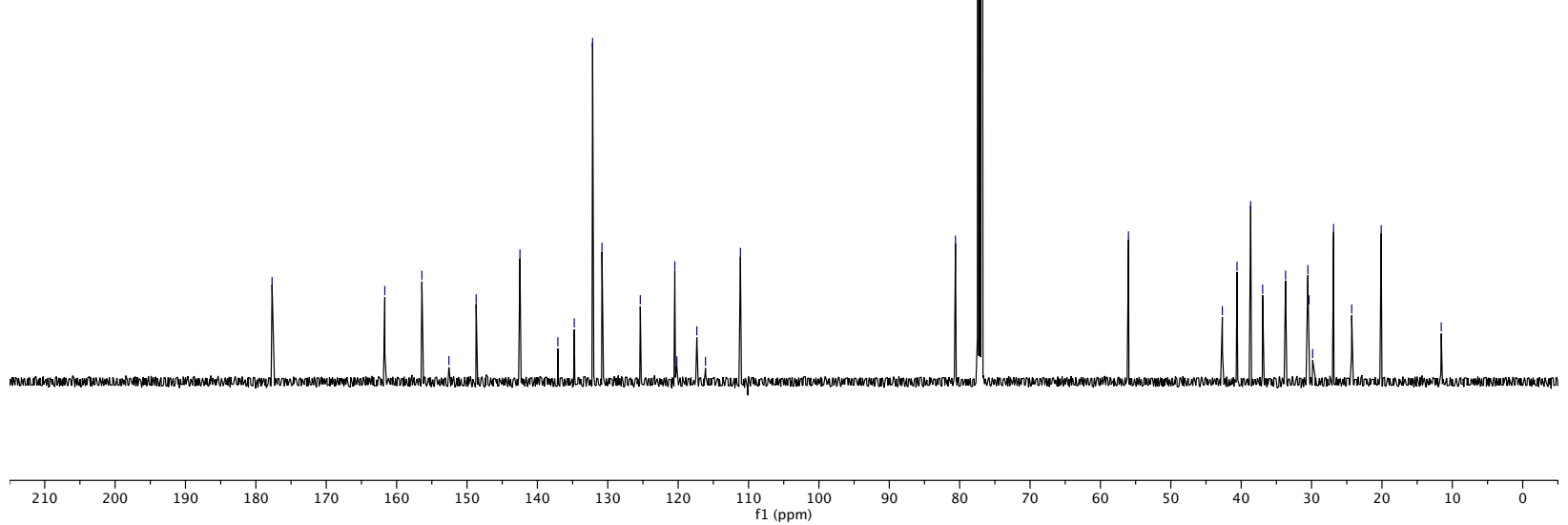


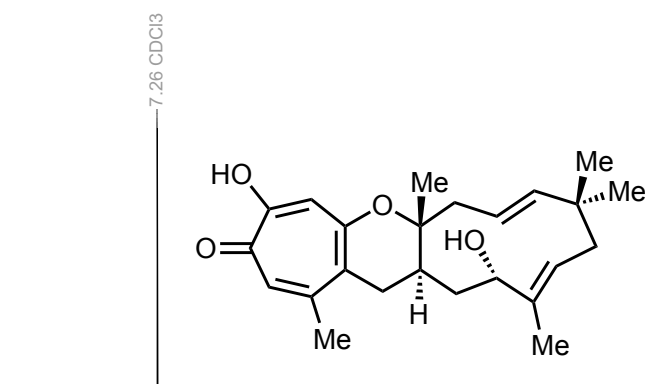

(-)-epolone B (ent-4)

$\left({ }^{1} \mathrm{H} \mathrm{NMR}, \mathrm{CDCl}_{3}, 500 \mathrm{MHz}\right)$
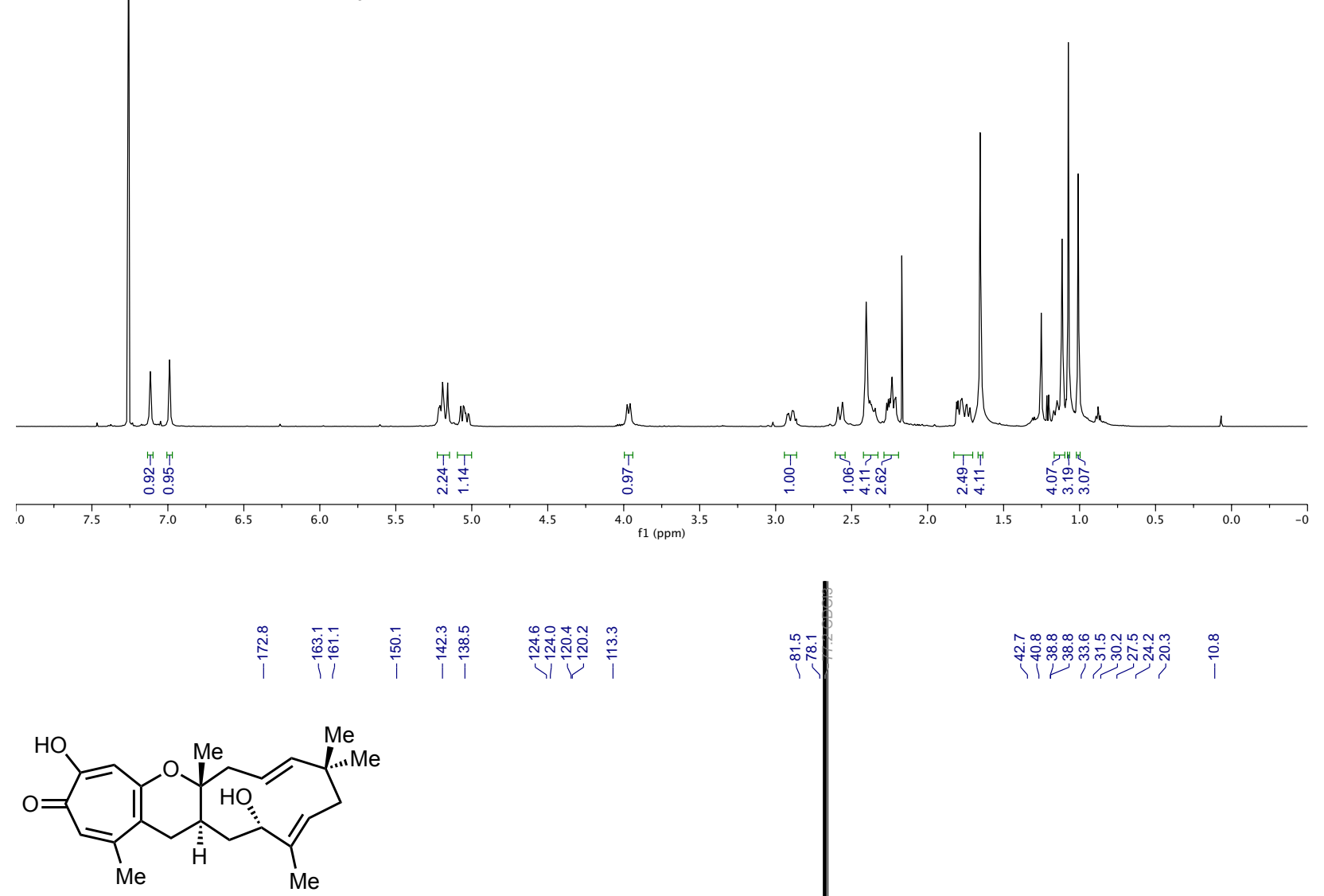

(-)-epolone B (ent-4)

$\left({ }^{13} \mathrm{C} \mathrm{NMR}, \mathrm{CDCl}_{3}, 126 \mathrm{MHz}\right)$
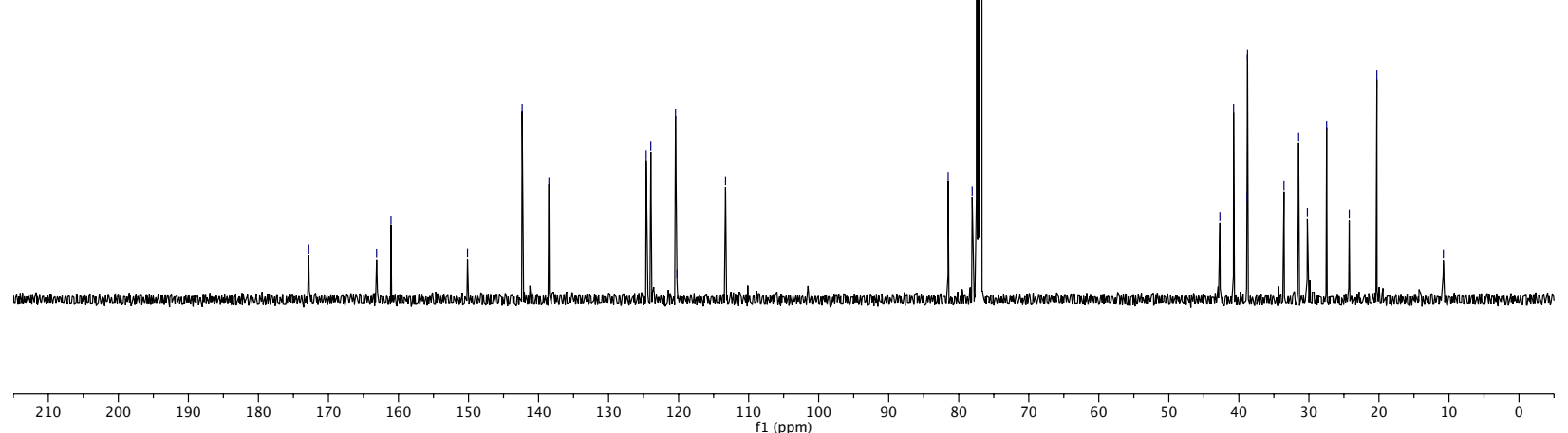


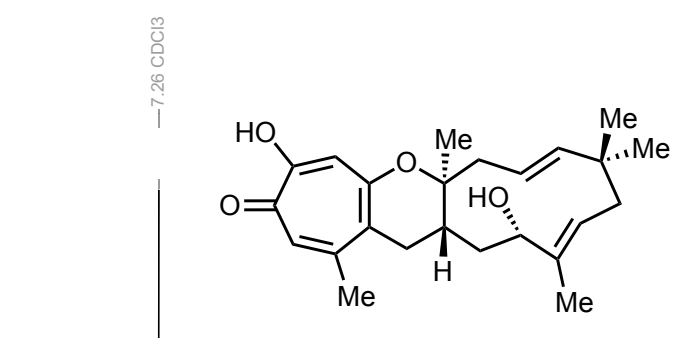

(+)-isoepolone B (ent-16)

$\left({ }^{1} \mathrm{H} \mathrm{NMR}, \mathrm{CDCl}_{3}, 500 \mathrm{MHz}\right)$

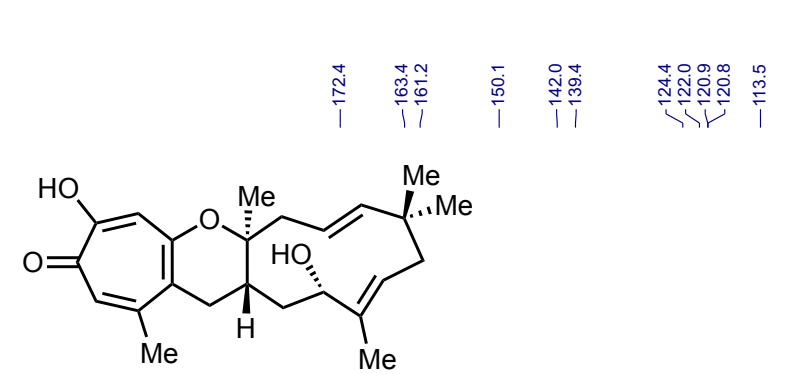

(+)-isoepolone B (ent-16)

$\left({ }^{13} \mathrm{C} \mathrm{NMR}, \mathrm{CDCl}_{3}, 126 \mathrm{MHz}\right)$
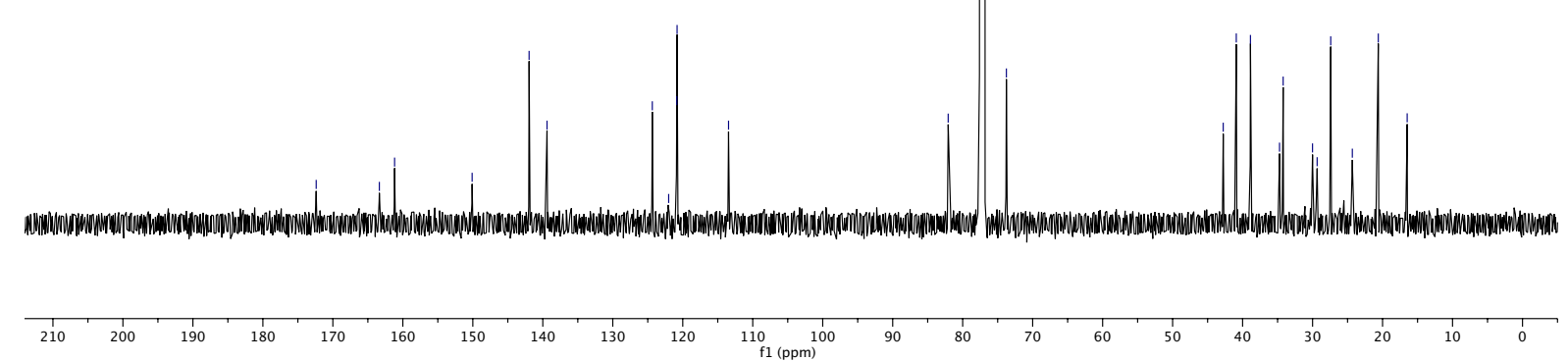


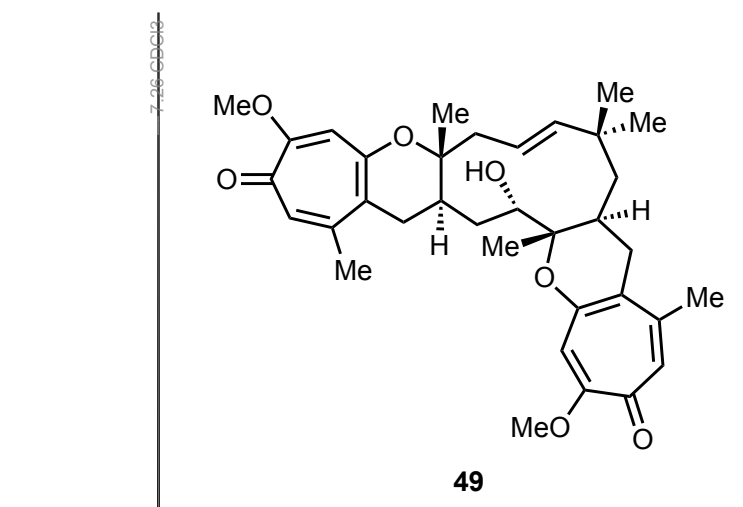

$\left({ }^{1} \mathrm{H} \mathrm{NMR}, \mathrm{CDCl}_{3}, 500 \mathrm{MHz}\right)$
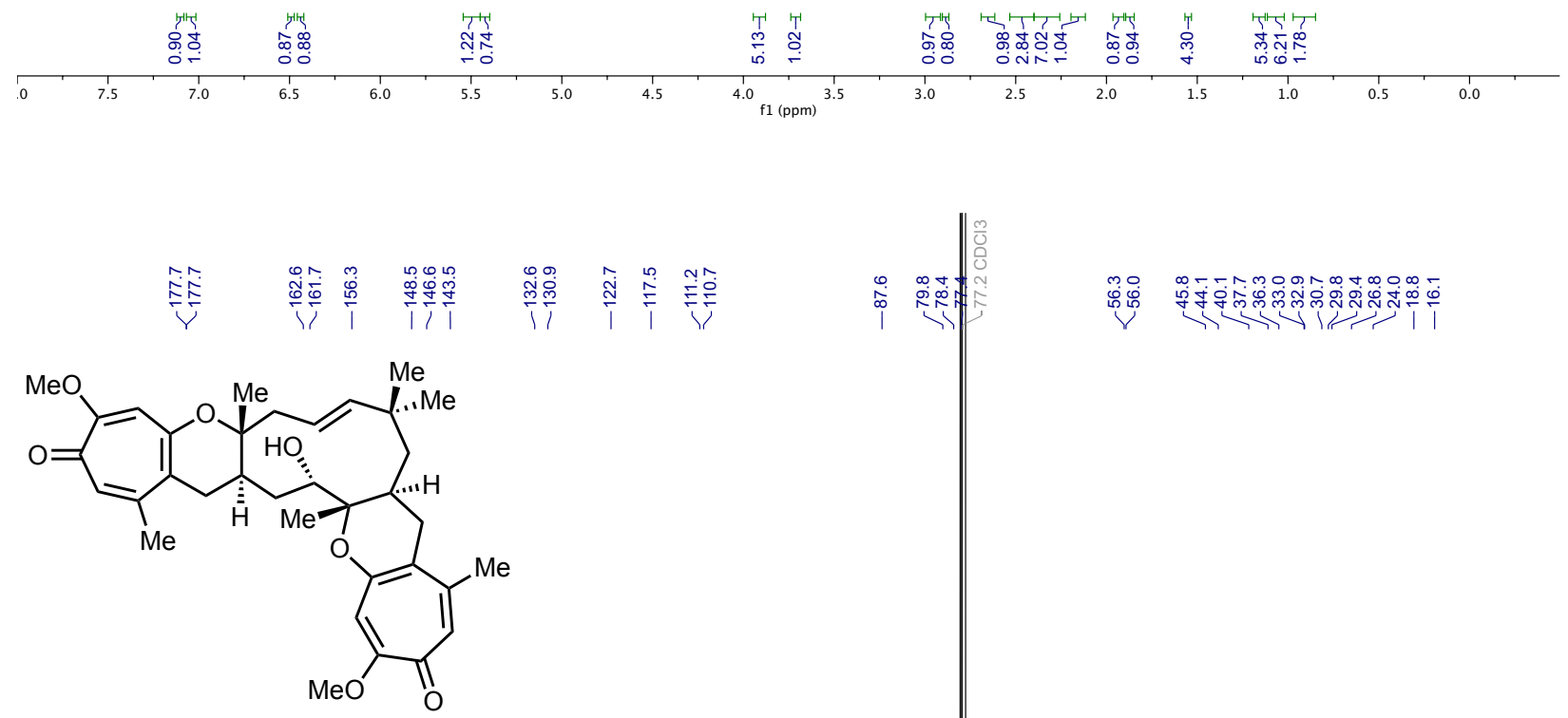

49

$\left({ }^{13} \mathrm{C} \mathrm{NMR}, \mathrm{CDCl}_{3}, 126 \mathrm{MHz}\right)$

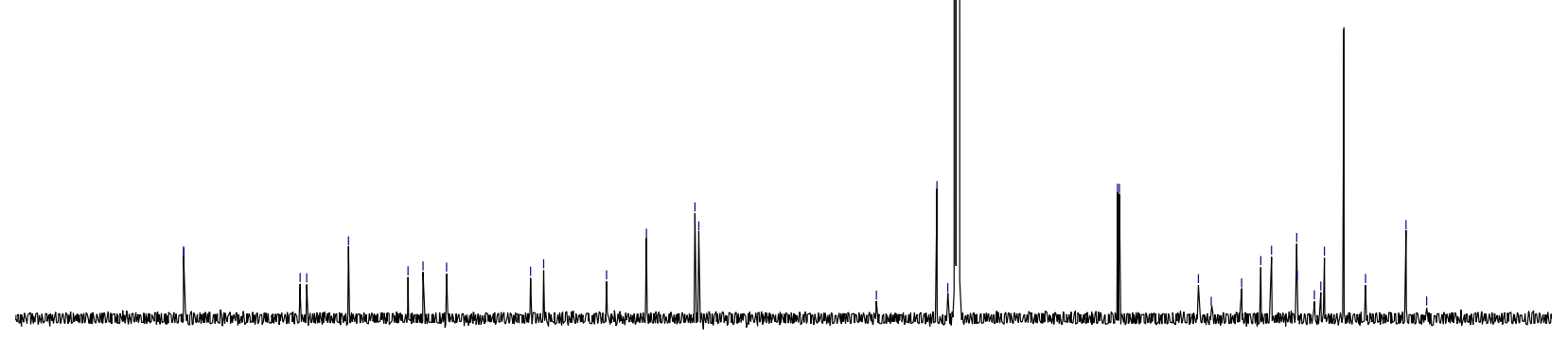

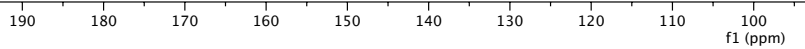



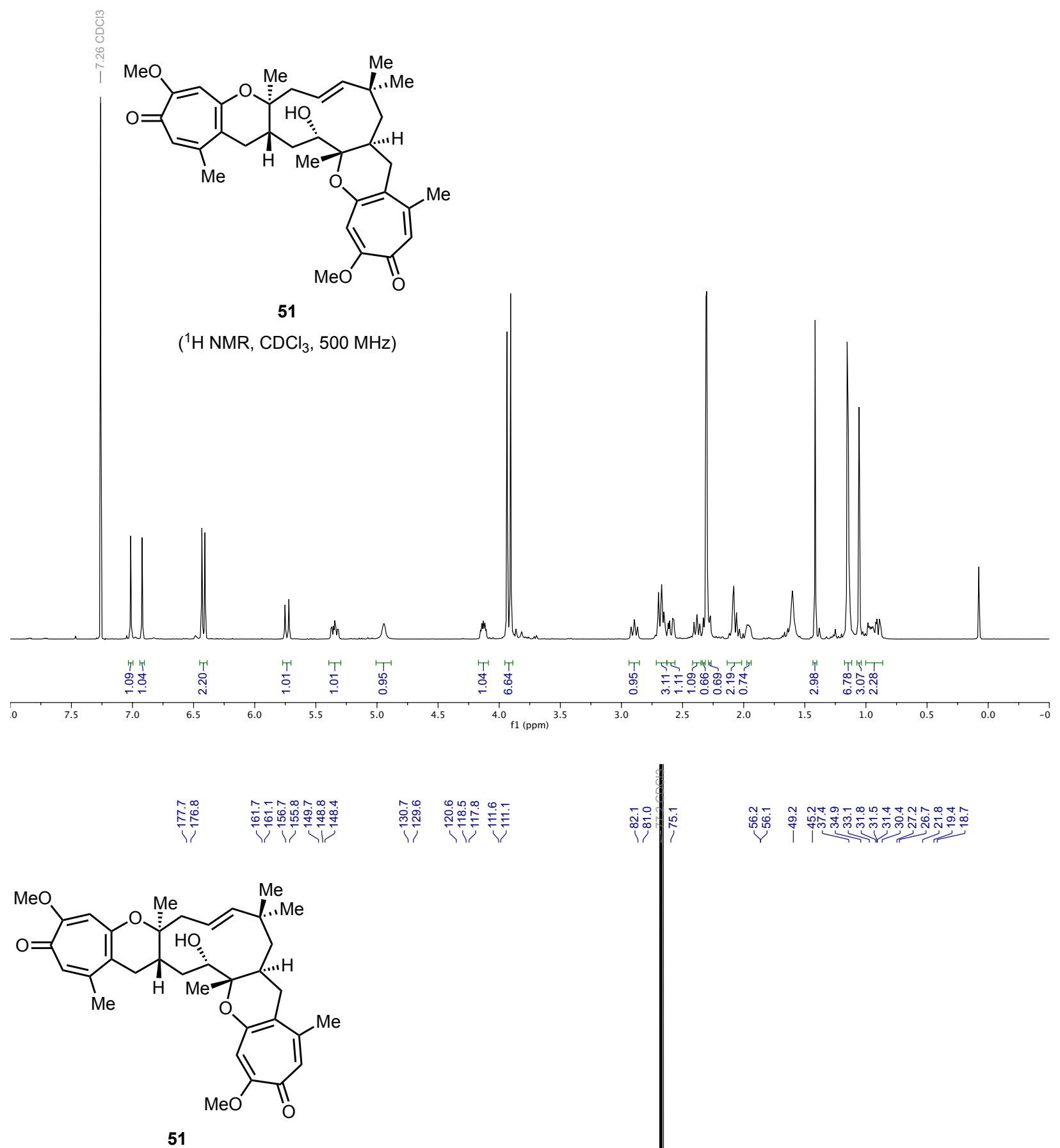

$\left({ }^{13} \mathrm{C} \mathrm{NMR}, \mathrm{CDCl}_{3}, 126 \mathrm{MHz}\right)$
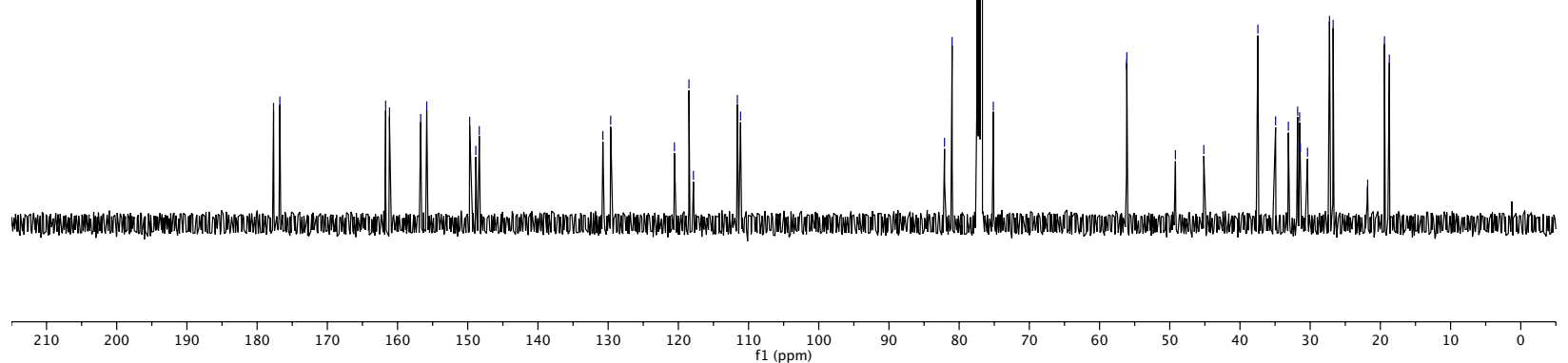

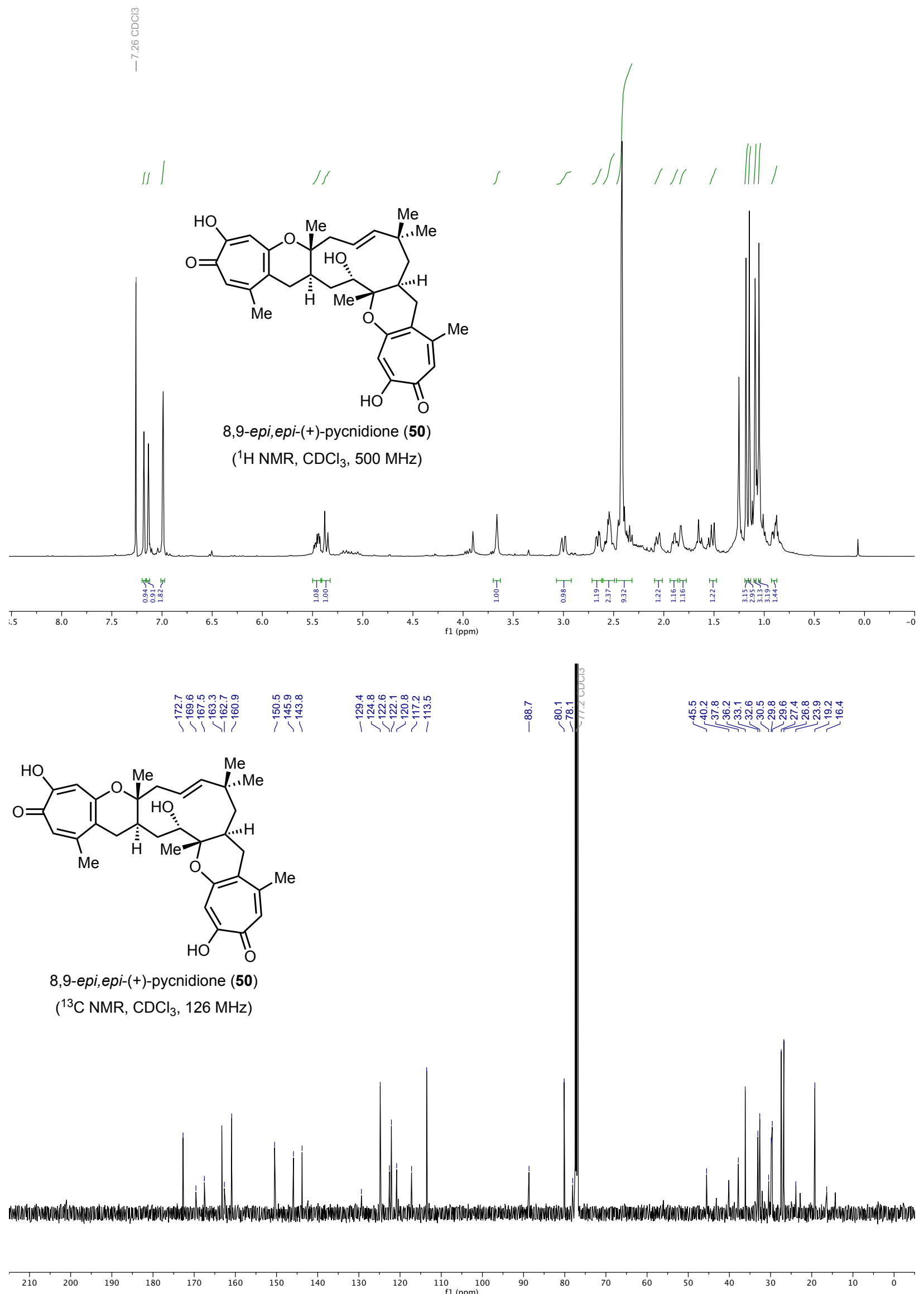


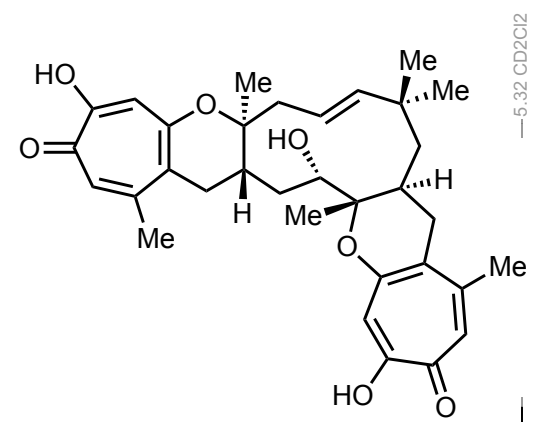

10-epi-(-)-pycnidione (52)

( ${ }^{1} \mathrm{H} \mathrm{NMR}, \mathrm{CD}_{2} \mathrm{Cl}_{2}, 500 \mathrm{MHz}$ )
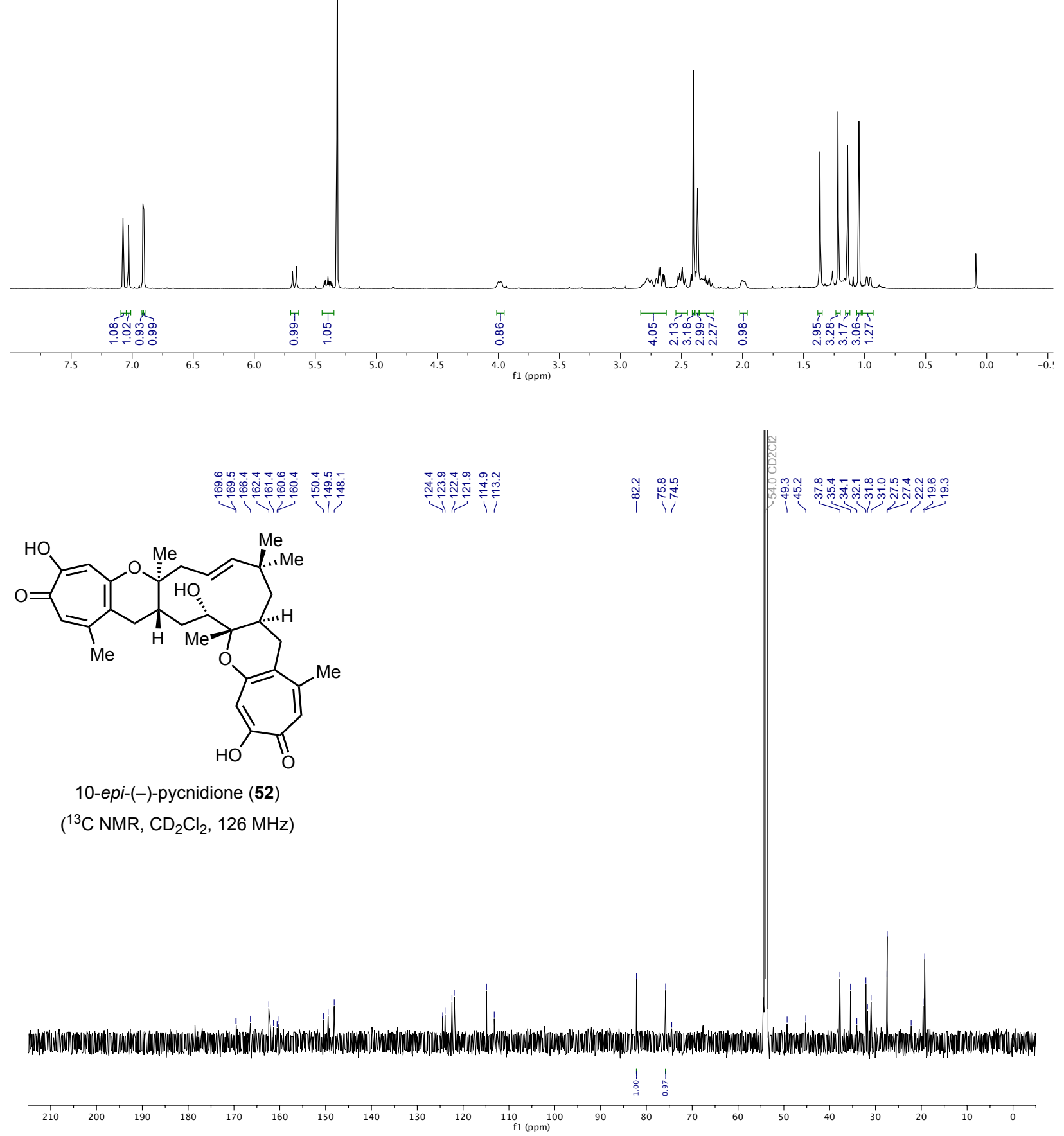


\section{References}

${ }^{1}$ Lange, G. L.; Demayo, P., Photochemical Synthesis - Stipitotanic Acid. Chem. Comm. 1967, 14, 704.

${ }^{2}$ Cai, Z.; Yongpruksa, N.; Harmata, M., Total Synthesis of the terpenoid Buddledone A: 11-Membered Ring-Closing Metathesis. Org. Lett. 2012, 14 , 1661.

${ }^{3}$ Elliott, M. R.; Dhimane, A. L.; Malacria, M., Biomimetic Diastereoselective Total Synthesis of Epi-Illudol via a Transannular Radical Cyclization Strategy. J. Am. Chem. Soc. 1997, 119, 3427-3428.

4 (a) Sakamoto, I.; Nishii, T.; Ozaki, F.; Kaku, H.; Tanaka, M.; Tsunoda, T., Preparation of (Cyanomethylene)tributylphosphorane: a New Mitsunobu-Type Reagent. Chem. Pharm. Bull. 2005, 53, 1508-1509;

(b) Sakamoto, I.; Kaku, H.; Tsunoda, T., Preparation of (Cyanomethylene)trimethylphosphorane as a New MitsunobuType Reagent. Chem. Pharm. Bull. 2003, 51, 474-476.

${ }^{5}$ Boivin, J.; Henriet, E.; Zard, S. Z., A Highly Efficient Reaction for the Synthesis of Esters and for the Inversion of Secondary Alcohols. J. Am. Chem. Soc. 1994, 116, 9739-9740.

Shintou, T.; Kikuchi, W.; Mukaiyama, T., Efficient Method for the Preparation of Carboxylic Acid Alkyl Esters or Alkyl Phenyl Ethers by a New-Type of Oxidation-Reduction Condensation Using 2,6-Dimethyl-1,4-benzoquinone and Alkoxydiphenylphosphines. B. Chem. Soc. Jpn. 2003, 76, 1645-1667.

${ }^{6}$ Shimizu, T.; Hiranuma, S.; Nakata, T., Efficient Method for Inversion of Secondary Alcohols by Reaction of Chloromethanesulfonates with Cesium Acetate. Tetrahedron Lett. 1997, 38, 3655-3655.

${ }^{7}$ Bairamov, G. I. Synthesis of New Nitrogen-Containing Compounds on the Basis of 2,6-Dichloro-5-oxo-2-hexene and their Study. Azerb. Khim. Zh., 2008, 3, 174-180.

${ }^{8}$ Hatch, L. W.; Chiola, V. The Preparation and Properties of 3-Chloro-1-propyne and 1-Chloro-2-butyne. J. Am. Chem. Soc., 1951, 73, 360-362.

${ }^{9}$ Bume, D. D.; Pitts, C. R.; Ghorbani, F.; Harry, S. A.; Capilato, J. N.; Siegler, M. A.; Lectka, T., Ketones as Directing Groups in Photocatalytic sp(3) C-H Fluorination. Chem. Sci. 2017, 8, 6918-6923.

${ }^{10}$ Crossley, S. W. M.; Barabe, F.; Shenvi, R. A., Simple, Chemoselective, Catalytic Olefin Isomerization. J. Am. Chem. Soc. 2014, 136, 16788-16791.

${ }^{11}$ Nakagiri, T.; Murai, M.; Takai, K., Stereospecific Deoxygenation of Aliphatic Epoxides to Alkenes under Rhenium Catalysis. Org. Lett. 2015, 17, 3346-3349.

${ }^{12}$ Chen, Q. B.; Gao, J.; Jamieson, C.; Liu, J. W.; Ohashi, M.; Bai, J.; Yan, D. J.; Liu, B. Y.; Che, Y. S.; Wang, Y. A.; Houk, K. N.; Hu, Y. C., Enzymatic Intermolecular Hetero-Diels-Alder Reaction in the Biosynthesis of Tropolonic Sesquiterpenes. J. Am. Chem. Soc. 2019, 141, 14052-14056.

${ }^{13}$ (a) Grimme, S.; Bannwarth, C.; Dohm, S.; Hansen, A.; Pisarek, J.; Pracht, P.; Seibert, J.; Neese, F. Fully Automated Quantum-Chemistry-Based Computation of Spin-Spin-Coupled Nuclear Magnetic Resonance Spectra. Angew. Chem. Int. Ed., 2017, 56, 14763-14769. (b) Grimme, S. Exploration of Chemical Compound, Conformer, and Reaction Space with Meta-Dynamics Simulations Based on Tight-Binding Quantum Chemical Calculations. J. Chem. Theory Comput., 2019, 155, 2847-2862. (c) Pracht, P.; Grimme, S. in preparation. (d) Grimme, S.; Bannwarth, C.; Shushkov, A Robust and Accurate Tight-Binding Quantum Chemical Method for Structures, Vibrational Frequencies, and Noncovalent Interactions of Large Molecular Systems Parametrized for All spd-Block Elements $(Z=1-86)$. P. $J$. Chem. Theory Comput. 2017, 13, 1989-2009. (e) C. Bannwarth, C.; Ehlert, S.; Grimme, S. J. GFN2-xTB-An Accurate and Broadly Parametrized Self-Consistent Tight-Binding Quantum Chemical Method with Multipole 
Electrostatics and Density-Dependent Dispersion Contributions. Chem. Theory Comput., 2019, 15, 1652-1671. (f) Pracht, P.; Caldeweyher, E.; Ehlert, S.; Grimme, S. ChemRxiv, 2019, preprint. DOI: 10.26434/chemrxiv.8326202.v1 14 (a) Chai, J. D.; Head-Gordon, M.; Phys. Chem. Chem. Phys. 2008, 10, 6615-6620. (b) Grimme, S.; Antony, J.; Ehrlich, S.; Krieg, H. J. Chem. Phys. 2010, 132, 154104. (c) Hariharan, P. C.; Pople, J. A. The Influence of Polarization Functions on Molecular Orbital Hydrogenation Energies. Theor. Chim. Acta 1973, 28, 213-222. (d) Hehre, W. J.; Ditchfield, R.; Pople, J. A. Self - Consistent Molecular Orbital Methods. XII. Further Extensions of Gaussian-Type Basis Sets for Use in Molecular Orbital Studies of Organic Molecules. J. Chem. Phys. 1972, 56, 2257-2261. (e) M. J. Frisch, et al. Gaussian 16 Revision A.03. 2016.

15 (a) Barone, V.; Cossi, M. Quantum Calculation of Molecular Energies and Energy Gradients in Solution by a Conductor Solvent Model. J. Phys. Chem. A 1998, 102, 1995-2001. (b) Cossi, M.; Rega, N.; Scalmani, G.; Barone, V. J. Comput. Chem. 2003, 24, 669-681. (c) Weigend, F.; Ahlrichs, R. Balanced Basis Sets of Split Valence, Triple Zeta Valence and Quadruple Zeta Valence Quality for H to Rn: Design and Assessment of Accuracy. Phys. Chem. Chem. Phys. 2005, 7, 3297-3305.

${ }^{16}$ (a) G. Luchini, J. V. Alegre-Requena, Y. Guan, I. Funes-Ardoiz, R. S. Paton GoodVibes: GoodVibes 3.0.0, 2019, http://doi.org/10.5281/zenodo.595246. (b) Grimme, S. Supramolecular Binding Thermodynamics by DispersionCorrected Density Functional Theory. Chem. Eur. J., 2012, 18, 9955-9964. b) Y. Li, Y.; Gomes, J.; Sharada, S. M.; Bell, A. T.; Head-Gordon, M. J. Phys. Chem. C, 2015, 119, 1840-1850.

17 (a) Zhao, Y.; Truhlar, D. G. The M06 Suite of Density Functionals for Main Group Thermochemistry, Thermochemical Kinetics, Noncovalent Interactions, Excited sSates, and Transition Elements: Two New Functionals and Systematic Testing of Four M06-Class Functionals and 12 Other Functionals. Theor. Chem. Acc. 2008, 120, 215241. (b) Mardirossian, N.; Head-Gordon, M. Thirty Years of Density Functional Theory in Computational Chemistry: an Overview and Extensive Assessment of 200 Density Functionals. Mol. Phys. 2017, 115, 2315-2372.

${ }^{18}$ Marenich, A. V; Cramer, C. J.; Truhlar, D. G. Universal Solvation Model Based on Solute Electron Density and on a Continuum Model of the Solvent Defined by the Bulk Dielectric Constant and Atomic Surface Tensions. J. Phys. Chem. B 2009, 113, 6378-6396.

${ }^{19}$ (a) Neese, F. The ORCA Program System. Wiley Interdiscip. Rev. Comput. Mol. Sci. 2012, 2, 73-78. (b) Neese, F. Software Update: The ORCA Program System, Version 4.0. Wiley Interdiscip. Rev. Comput. Mol. Sci. 2018, 8, e1327.

${ }^{20}$ Boyko, Y. D.; Huck, C. J.; Ning, S.; Shved, A. S.; Yang, C.; Chu, T.; Tonogai, E. J.; Hergenrother, P. J.; Sarlah, D. Synthetic Studies on Selective, Proapoptotic Isomalabaricane Triterpenoids Aided by Computational Techniques. $J$. Am. Chem. Soc. 2021, 143, 2138-2155.

${ }^{21}$ Grimme, S.; Brandenburg, J. G.; Bannwarth, C.; Hansen, A. Consistent Structures and Interactions by Density Functional Theory with Small Atomic Orbital Basis Sets. J. Chem. Phys. 2015, 143, 054107.

${ }^{22}$ Dolomanov, O. V.; Bourhis, L. J.; Gildea, R.J.; Howard, J. A. K.; Puschmann, H. OLEX2: a Complete Structure Solution, Refinement and Analysis Program. J. Appl. Cryst. 2009, 42, 339-341.

${ }^{23}$ Sheldrick, G. M. Acta Cryst. 2015, A71, 3-8.

${ }^{24}$ Sheldrick, G. M. Acta Cryst. 2008, A64, 112-122.

${ }^{25}$ Harris, G. H.; Hoogsteen, K.; Silverman, K. C.; Raghoobar, S. L.; Bills, G. F.; Lingham, R. B.; Smith, J. L.; Dougherty, H. W.; Cascales, C.; Pelaez, F., Isolation and Structure Determination of Pycnidione, a Novel Bistropolone Stromelysin Inhibitor from a Phoma sp. Tetrahedron 1993, 49, 2139-2144. 
${ }^{26}$ Cai, P.; Smith, D.; Cunningham, B.; Brown-Shimer, S.; Katz, B.; Pearce, C.; Venables, D.; Houck, D. Epolones: Novel Sesquiterpene-Tropolones from Fungus OS-F69284 That Induce Erythropoietin in Human Cells. J. Nat. Prod. 1998, 61, 791-795.

${ }^{27}$ Overy, D. P.; Berrue, F.; Correa, H.; Hanif, N.; Hay, K.; Lanteigne, M.; Mquilian, K.; Duffy, S.; Boland, P.; Jagannathan, R.; Carr, G. S.; Vansteeland, M.; Kerr, R. G. Sea foam as a Source of Fungal Inoculum for the Isolation of Biologically Active Natural Products. Mycology 2014, 5, 130-144. 